INEL/95-0135-Vol, 3

\title{
A Comprehensive Inventory of Radiological and Nonradiological Contaminants in Waste Buried or Projected to be Buried in the Subsurface Disposal Area of the INEL RWMC During the Years 1984-2003
}

\section{Volume 3}

Published May 1995

Idaho National Engineering Laboratory Lockheed Idaho Technologies Company Idaho Falls, Idaho 83415 
$\therefore$; $\Rightarrow$ i 


\section{DISCLAIMER}

Portions of this document may be illegible in electronic image products. Images are produced from the best available original document. 


\section{PREFACE}

This report, $A$ Comprehensive Inventory of Radiological and Nonradiological Contaminants in Waste Buried or Projected to be Buried in the Subsurface Disposal Area of the INEL RWMC During the Years 1984-2003, is composed of three volumes. Volume 1 consists of the main body of the report and Appendices $A, C, D, E, F$, and G. Appendix B, the complete printout of the inventory database, is provided in Volumes 2 and 3. Because of its size, distribution of Appendix $B$ has been limited. A copy of the volumes containing Appendix B can be provided on request.

\section{DISCLAIMER}

This report was prepared as an account of work sponsored by an agency of the United States Government. Neither the United States Government nor any agency thereof, nor any of their employees, makes any warranty, express or implied, or assumes any legal liability or responsibility for the accuracy, completeness, or usefulness of any information, apparatus, product, or process disclosed, or represents that its use would not infringe privately owned rights. Reference herein to any specific commercial product, process, or service by trade name, trademark, manufacturer, or otherwise does not necessarily constitute or imply its endorsement, recommendation, or favoring by the United States Government or any agency thereof. The views and opinions of authors expressed herein do not necessarily state or reflect those of the United States Government or any agency thereof. 


\title{
Appendix B
}

Complete Printout of Contaminant Inventory and Other Information from the CIDRA Database

\author{
(continued)
}



DATA INPUT FOR HISTORICAL DATA TASK FOR RWMC SUBSURFACE DISPOSAL AREA

PART A - GENERAL INFORMATION RDT - 268

1. Preparer: Amaro, C.

3. Generator: TRA (area or contractor - use code from attached list)

5. Number of waste stream from this facility: $1 \mathrm{R}$

7. Type of radioactive waste (check box):

[ ] TRU or suspect TRU

[X] LLW

] non-radioactive

3. Actual years disposed of at SDA: starting year 1984 Ending year 1993
2. Date prepared: $07 / 03 / 94$

4. Particular facility: 603

(building number - use code from attached list)

6. Waste stream:

Resin.

9. Waste stream volume:

Amount

451.0130 Units Cubic meters.

check box: [ ] annual or [X] total over all years

Check box: [ ] container volume or [X] waste volume

10. Comments (specify number of pertinent question):

4. Also 670 and 632 . 
General physical form (see attached list) 2. Details on physical form(particularly confinement related) sin. ] other (specify)

Chemical form: lknown.

Waste container type (see attached list) her.

Comments (specify number of pertinent question): 5. Also RD5, $O$ is a cask.
4. Inner packaging: [ ] plastic bag [ ] plastic liner [ ] metal liner [ ] none [X] other (specify) other.

6. Other characteristics of interest: 


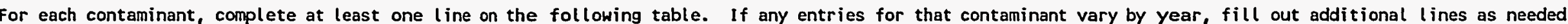

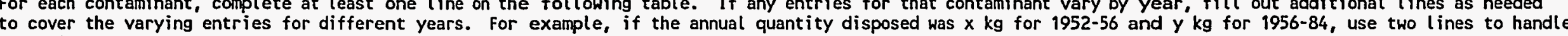
this situation.

\begin{tabular}{|c|c|c|c|c|c|c|c|c|c|c|}
\hline $\begin{array}{l}\text { Contaminant } \\
\text { \& CAS Registry Number }\end{array}$ & Physical form & Chemical Form & $\begin{array}{l}\text { (A)nnual/(T)otal } \\
\text { Quantity }\end{array}$ & Unit & $\begin{array}{l}\text { Begin } \\
\text { Year }\end{array}$ & $\begin{array}{l}\text { End } \\
\text { Year }\end{array}$ & $\begin{array}{l}\text { Samp } \\
\text { les? }\end{array}$ & $\begin{array}{c}\text { Minimum } \\
\text { Value/\#Samp }\end{array}$ & $\begin{array}{r}\text { Maximum } \\
\text { Value/STD }\end{array}$ & $\begin{array}{l}\text { Basis for } \\
\text { Uncertainty }\end{array}$ \\
\hline \multicolumn{11}{|l|}{ None. } \\
\hline & & ' & & & & & & & & \\
\hline & & & & & & & & & & \\
\hline & & & & & & & & & & \\
\hline & & & & & & & & & & \\
\hline & & & & & & & & & & \\
\hline & & & & & & & & & & \\
\hline & & & & & & & & & & \\
\hline & & & & & & & & & & \\
\hline & & & & & & & & & & \\
\hline & & & & & & & & & & \\
\hline & & & & & & & & & & \\
\hline & & & & & & & & & & \\
\hline & & & & & & & & & & \\
\hline & & & & & & & & & & \\
\hline & & & & & & & & & & \\
\hline & & & & & & & & & & \\
\hline
\end{tabular}

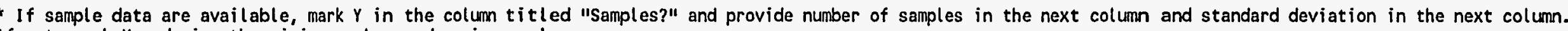
if not, mark $N$ and give the minimum value and maximum value.

idditional information or explanations (indicate pertinent contaminant) 
each contaminant, complete at least one line on the following table. If any entries for that contaminant vary by year, fill out additional lines as needed over the varying entries for different years. For example, if the annual quantity disposed was $x \mathrm{~kg}$ for $1952-56$ and $y \mathrm{~kg}$ for $1956-84$, use two lines to handle situation.

\begin{tabular}{|c|c|c|c|c|c|c|c|c|c|c|}
\hline Radionuclide & Physical Form & Chemical Form & $\begin{array}{l}\text { (A) nnual/(T)otal } \\
\text { Quantity }\end{array}$ & Unit & $\begin{array}{l}\text { Begin } \\
\text { Year }\end{array}$ & $\begin{array}{l}\text { End } \\
\text { Year }\end{array}$ & $\begin{array}{l}\text { Samp } \\
\text { les? }\end{array}$ & $\begin{array}{c}\text { Minimum } \\
\text { value/\#Samp }\end{array}$ & $\begin{array}{r}\text { Maximum } \\
\text { Value/STD }\end{array}$ & $\begin{array}{l}\text { Basis for } \\
\text { Uncertainty }\end{array}$ \\
\hline$H-3$ & Resin. & Unknown. & T. . .02564000000000 & CI & 1984 & 1984 & N & & & See continuation page. \\
\hline$c-14$ & Resin. & Unknown. & T.22050000000000 & CI & 1984 & 1984 & $\mathrm{~N}$ & & & See continuation page. \\
\hline $\mathrm{Fe}-55$ & Resin. & Unknown. & $T 10.260000000000$ & CI & 1984 & 1984 & N & & & See continuation page. \\
\hline $\mathrm{Ni}-59$ & Resin. & Unknown. & T. .14360000000000 & CI & 1984 & 1984 & N & & & See continuation page. \\
\hline $\mathrm{Ni}-63$ & Resin. & Unknown. & T 14.360000000000 & CI & 1984 & 1984 & N & & & See continuation page. \\
\hline Co- 60 & Resin. & Unknown. & T 34.880000000000 & CI & 1984 & 1984 & $\mathrm{~N}$ & & & See continuation page. \\
\hline $\mathrm{Sr}-90$ & Resin. & Unknown. & T 14.360000000000 & CI & 1984 & 1984 & N & & & See continuation page. \\
\hline Tc-99 & Resin. & Unknown. & T.00076930000000 & CI & 1984 & 1984 & N & & & See continuation page. \\
\hline $1-129$ & Resin. & Unknown. & T. .00000318000000 & $\mathrm{CI}$ & 1984 & 1984 & N & & & See continuation page. \\
\hline
\end{tabular}

sample data are available, mark $Y$ in the column titled "Samples?" and provide number of samples in the next column and standard deviation in the next column. ot, mark $N$ and give the minimum value and maximum value.

tional information or explanations (indicate pertinent contaminant)

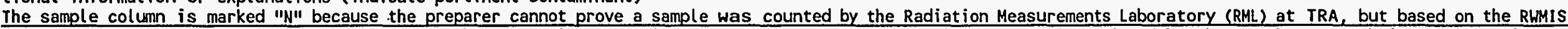

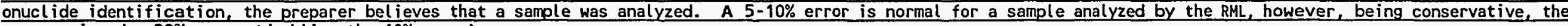
varer assigned a $20 \%$ error (doubl ing the 10\% error)

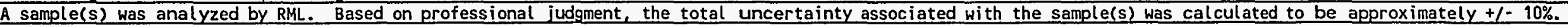


or each contaminant, complete at least one line on the following table. If any entries for that contaminant vary by year, fill out additional lines as needed 0 cover the varying entries for different years. For example, if the annual quantity disposed was $x \mathrm{~kg}$ for 1952-56 and $y$ kg for $1956-84$, use two lines to handle his situation.

\begin{tabular}{|c|c|c|c|c|c|c|c|c|c|c|}
\hline Radionucl ide & Physical Form & Chemical Form & $\begin{array}{l}\text { (A)nnual/(T)otal } \\
\text { Quantity }\end{array}$ & Unit & $\begin{array}{l}\text { Begin } \\
\text { Year }\end{array}$ & $\begin{array}{l}\text { End } \\
\text { Year }\end{array}$ & $\begin{array}{l}\text { Samp } \\
\text { les? }\end{array}$ & $\begin{array}{c}\text { Minimum } \\
\text { Value/\#Samp }\end{array}$ & $\begin{array}{r}\text { Maximem } \\
\text { Value/STD }\end{array}$ & $\begin{array}{l}\text { Basis for } \\
\text { Uncertainty }\end{array}$ \\
\hline Cs-137 & Resin. & Unknown. & T 15.900000000000 & CI & 1984 & 1984 & H & & & See continuation page. \\
\hline Ce-144 & Resin. & Unknown. & T. .39490000000000 & CI & 1984 & 1984 & N & & & See cont inuation page. \\
\hline Eu-154 & Resin. & Unknown. & T. .37440000000000 & CI & 1984 & 1984 & N & & & See continuation page. \\
\hline Eu- 155 & Resin. & Unknown. & T. .15900000000000 & CI & 1984 & 1984 & N & & & See continuation page. \\
\hline$U-234$ & Resin. & Unknown. & $T .00021540000000$ & $\mathrm{CI}$ & 1984 & 1984 & N & & & See cont inuation page. \\
\hline$U-235$ & Resin. & Unknown. & T. .00000471800000 & CI & 1984 & 1984 & N & & & See cont inuation page. \\
\hline$U-236$ & Resin. & Unknown. & T. .00008719000000 & CI & 1984 & 1984 & N & & & See cont inuation page. \\
\hline Np-237 & Resin. & Unknown. & $\mathrm{T} .00013330000000$ & $\mathrm{Cl}$ & 1984 & 1984 & N & & & See continuation page. \\
\hline Pu-238 & Resin. & Unknown. & T. .00923200000000 & CI & 1984 & 1984 & N & & & See continuation page. \\
\hline
\end{tabular}

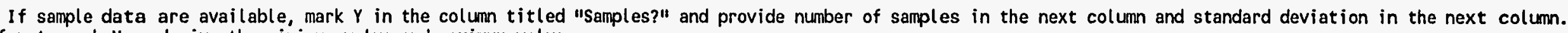
f not, mark $N$ and give the minimum value and maximum value.

dditional information or explanations (indicate pertinent contaminant)

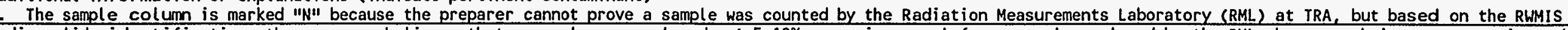

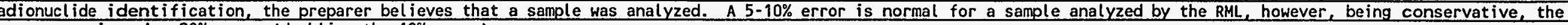
reparer assigned a $20 \%$ error (doubling the $10 \%$ error).

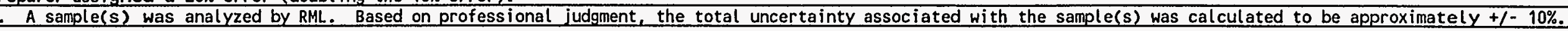


each contaminant, complete at least one line on the following table. If any entries for that contaminant vary by year, fill out additional lines as needed cover the varying entries for different years. For example, if the annual quantity disposed was $x \mathrm{~kg}$ for $1952-56$ and $y$ kg for $1956-84$, use two lines to handle 3 situation.

\begin{tabular}{|c|c|c|c|c|c|c|c|c|c|c|}
\hline Radionuclide & Physical Form & Chemical Form & $\begin{array}{l}\text { (A)nnual } /(\mathrm{T}) \text { otal } \\
\text { Quant i ty }\end{array}$ & Unit & $\begin{array}{l}\text { Begin } \\
\text { Year }\end{array}$ & $\begin{array}{l}\text { End } \\
\text { Year }\end{array}$ & $\begin{array}{l}\text { Samp } \\
\text { les? }\end{array}$ & $\begin{array}{c}\text { Minimum } \\
\text { Value/\#Samp }\end{array}$ & $\begin{array}{r}\text { Maximum } \\
\text { value/STD }\end{array}$ & $\begin{array}{l}\text { Basis for } \\
\text { Uncertainty }\end{array}$ \\
\hline Pu-239 & Resin. & Unknown. & $T .00235900000000$ & CI & 1984 & 1984 & N & & & See continuation page. \\
\hline Pu-240 & Resin. & Unknown. & T. .00143600000000 & CI & 1984 & 1984 & N & & & See continuation page. \\
\hline Pu-241 & Resin. & Unknown. & T. .76930000000000 & CI & 1984 & 1984 & N & & & See continuation page. \\
\hline Am-241 & Resin. & Unknown. & T. .20510000000000 & CI & 1984 & 1984 & N & & & See continuation page. \\
\hline $\mathrm{Cm}-242$ & Resin. & Unknown. & T. .0143600000000 & CI & 1984 & 1984 & N & & & See continuation page. \\
\hline $\mathrm{Cm}-244$ & Resin. & Unknown. & $T .00666700000000$ & CI & 1984 & 1984 & N & & & See cont inuation page. \\
\hline$B a-140$ & Resin. & Unknown. & T. .96736000000000 & CI & 1984 & 1984 & N & $-20 \%$ & $+20 \%$ & See coment (a) below. \\
\hline $\mathrm{Ce}-141$ & Resin. & Unknown. & T. .92100000000000 & $\mathrm{CI}$ & 1984 & 1984 & N & $-20 \%$ & $+20 \%$ & See comment (a) below. \\
\hline Ce-144 & Resin. & Unknown. & T 4.4850000000000 & $\mathrm{CI}$ & 1984 & 1984 & N & $-20 \%$ & $+20 \%$ & See comment (a) below. \\
\hline
\end{tabular}

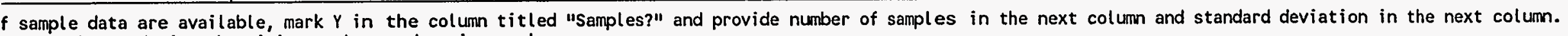
not, mark $N$ and give the minimum value and maximum value.

itional information or explanations (indicate pertinent contaminant)

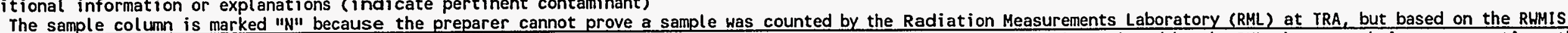

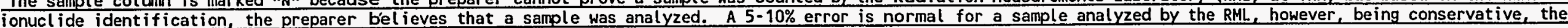
parer assigned a $20 \%$ error (doubling the $10 \%$ error).

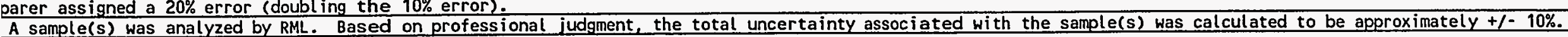


or each contaminant, complete at least one line on the following table. If any entries for that contaminant vary by year, fill out additional lines as needed o cover the varying entries for different years. For example, if the annual quantity disposed was $x \mathrm{~kg}$ for $1952-56$ and $y \mathrm{~kg}$ for $1956-84$, use two lines to handte his situation.

\begin{tabular}{|c|c|c|c|c|c|c|c|c|c|c|}
\hline Radionuclide & Physical form & Chemical form & $\begin{array}{l}\text { (A) nnual } /(\mathrm{T}) \text { otal } \\
\text { Quant i ty }\end{array}$ & Unit & $\begin{array}{l}\text { Begin } \\
\text { Year }\end{array}$ & $\begin{array}{l}\text { End } \\
\text { Year }\end{array}$ & $\begin{array}{l}\text { Samp } \\
\text { les? }\end{array}$ & $\begin{array}{c}\text { Minimum } \\
\text { Value/\#Samp }\end{array}$ & $\begin{array}{c}\text { Maximum } \\
\text { Value/STD }\end{array}$ & $\begin{array}{l}\text { Basis for } \\
\text { Uncertainty }\end{array}$ \\
\hline Co-58 & Resin. & Unknown. & T. .79048000000000 & $\mathrm{CI}$ & 1984 & 1984 & N & $-20 \%$ & $+20 \%$ & See comment (a) below. \\
\hline $\mathrm{Co}-60$ & Resin. & Unknown. & T 30.213100000000 & CI & 1984 & 1984 & N & $-20 \%$ & $+20 \%$ & See comment (a) below. \\
\hline $\mathrm{Cr}-51$ & Resin. & Unknown. & T 675.21000000000 & $\mathrm{Cl}$ & 1984 & 1984 & N & $-20 \%$ & $+20 \%$ & See comment (a) below. \\
\hline Cs-134 & Resin. & Unknown. & T. .29628000000000 & CI & 1984 & 1984 & $N$ & $-20 \%$ & $+20 \%$ & See comment (a) below. \\
\hline Cs -137 & Resin. & Unknown. & T. .26080000000000 & $\mathrm{Cl}$ & 1984 & 1984 & N & $-20 \%$ & $+20 \%$ & See comment (a) below. \\
\hline La- 140 & Resin. & Unknown. & T 1.0693600000000 & CI & 1984 & 1984 & N & $-20 \%$ & $+20 \%$ & See comment (a) below. \\
\hline$M n-54$ & Resin. & Unknown. & $\mathrm{T} .23848000000000$ & CI & 1984 & 1984 & N & $-20 \%$ & $+20 \%$ & See comment (a) below. \\
\hline $\mathrm{Nb}-95$ & Resin. & Unknown. & r. .7520000000000 & CI & 1984 & 1984 & N & $-20 \%$ & $+20 \%$ & See comment (a) below. \\
\hline $2 n-65$ & Resin. & Unknown. & $T .47600000000000$ & $\mathrm{CI}$ & 1984 & 1984 & N & $-20 \%$ & $+20 \%$ & See comment (a) below. \\
\hline
\end{tabular}

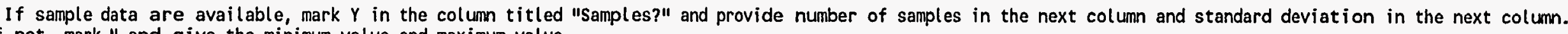
not, mark $N$ and give the minimum value and maximum value.

iditional information or explanations (indicate pertinent contaminant)

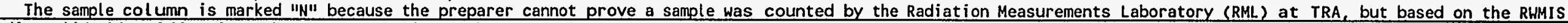

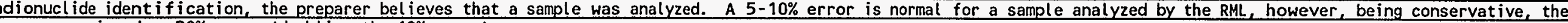
eparer assigned a $20 \%$ error (doubling the $10 \%$ error)

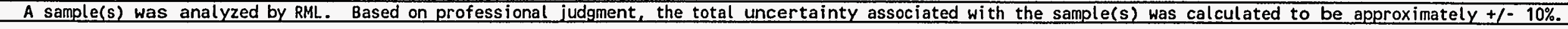


- each contaminant, complete at least one $l$ ine on the following table. If any entries for that contaminant vary by year, fill out additional lines as needed

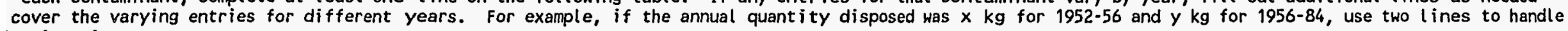
is situation.

\begin{tabular}{|c|c|c|c|c|c|c|c|c|c|c|}
\hline Radionuclide & Physical form & Chemical Form & $\begin{array}{l}\text { (A) nnual /(T)otal } \\
\text { Quant i ty }\end{array}$ & Unit & $\begin{array}{l}\text { Begin } \\
\text { Year }\end{array}$ & $\begin{array}{l}\text { End } \\
\text { Year }\end{array}$ & $\begin{array}{l}\text { Samp } \\
\text { les? }\end{array}$ & $\begin{array}{c}\text { Minimum } \\
\text { Value/\#Samp }\end{array}$ & $\begin{array}{r}\text { Maximum } \\
\text { value/STD }\end{array}$ & $\begin{array}{l}\text { Basis for } \\
\text { Uncertainty }\end{array}$ \\
\hline $2 r-95$ & Resin. & Unknown. & T 1.1280000000000 & CI & 1984 & 1984 & N & $-20 \%$ & $+20 \%$ & See comment (a) below. \\
\hline$H-3$ & Resin. & Unknown. & $T .01238000000000$ & CI & 1985 & 1985 & $\therefore \mathrm{N}$ & & & See continuation page. \\
\hline C- 14 & Resin. & Unknown. & $T .10650000000000$ & CI & 1985 & 1985 & N & & & See continuation page. \\
\hline $\mathrm{Fe}-55$ & Resin. & Unknown. & T 4.9540000000000 & CI & 1985 & 1985 & N & & & See continuation page. \\
\hline $\mathrm{Ni}-59$ & Resin. & Unknown. & $r .06935000000000$ & $\mathrm{CI}$ & 1985 & 1985 & N & & & See cont inuation page. \\
\hline $\mathrm{Ni}-63$ & Resin. & Unknown. & T 6.935000000000 & CI & 1985 & 1985 & N & & & See continuation page. \\
\hline Co- 60 & Resin. & Unknown. & T 16.840000000000 & CI & 1985 & 1985 & N & & & See continuation page. \\
\hline sr-90 & Resin. & Unknown. & T 6.935000000000 & CI & 1985 & 1985 & N & & & See continuation page. \\
\hline Tc- 99 & Resin. & Unknown. & T.00037150000000 & $\mathrm{CI}$ & 1985 & 1985 & N & & & See continuation page. \\
\hline
\end{tabular}

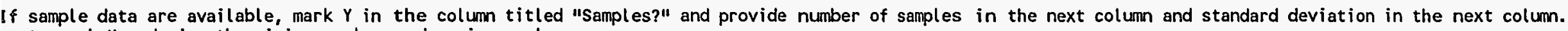
not, mark $N$ and give the minimum value and maximum value.

ditional information or explanations (indicate pertinent contaminant)

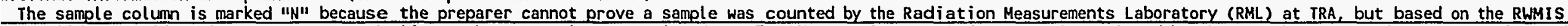

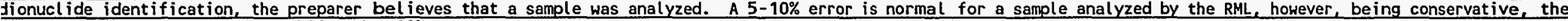
eparer assigned a $20 \%$ error (doubling the $10 \%$ error)

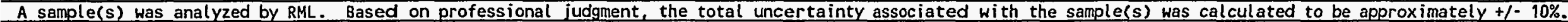




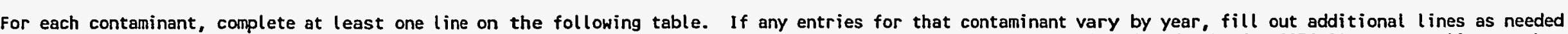

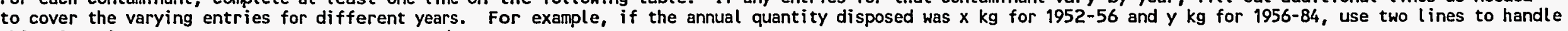
this situation.

\begin{tabular}{|c|c|c|c|c|c|c|c|c|c|c|}
\hline Radionuclide & Physical Form & Chemical Form & $\begin{array}{l}\text { (A)nnual/(T)otal } \\
\text { Quantity }\end{array}$ & Unit & $\begin{array}{l}\text { Begin } \\
\text { Year }\end{array}$ & $\begin{array}{l}\text { End } \\
\text { Year }\end{array}$ & $\begin{array}{l}\text { Samp } \\
\text { les? }\end{array}$ & $\begin{array}{c}\text { Minimum } \\
\text { Value/\#Samp }\end{array}$ & $\begin{array}{r}\text { Maximum } \\
\text { Value/STD }\end{array}$ & $\begin{array}{l}\text { Basis for } \\
\text { Uncertainty }\end{array}$ \\
\hline$I-129$ & Resin. & Unknown. & T. .00000153560000 & $\mathrm{Cl}$ & 1985 & 1985 & N & & & See continuation page. \\
\hline Cs -137 & Resin. & Unknown. & T 7.6780000000000 & CI & 1985 & 1985 & N & & & See continuation page. \\
\hline $\mathrm{Ce}-144$ & Resin. & Unknown. & $T .19070000000000$ & $\mathrm{CI}$ & 1985 & 1985 & $\mathbf{N}$ & & & See continuation page. \\
\hline Eu-154 & Resin. & Unknown. & $T .18080000000000$ & $\mathrm{CI}$ & 1985 & 1985 & N & & & See continuation page. \\
\hline Eu-155 & Resin. & Unknown. & T. .07678000000000 & $\mathrm{CI}$ & 1985 & 1985 & N & & & See continuation page. \\
\hline $\mathrm{U}-234$ & Resin. & Unknown. & T. .00010400000000 & $\mathrm{CI}$ & 1985 & 1985 & $\mathbf{N}$ & & & See continuation page. \\
\hline U-235 & Resin. & Unknown. & $T .00000227900000$ & CI & 1985 & 1985 & N & & & See continuation page. \\
\hline$U-236$ & Resin. & Unknown. & T. .00004211000000 & CI & 1985 & 1985 & N & & & See continuation page. \\
\hline Np-237 & Resin. & Unknown. & $T .00006440000000$ & CI & 1985 & 1985 & $\mathbf{N}$ & & & See continuation page. \\
\hline
\end{tabular}

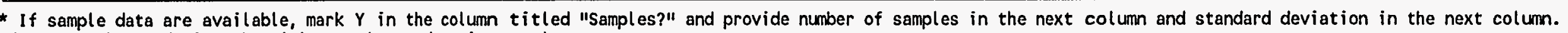
If not, mark $N$ and give the minimum value and maximum value.

Additional information or explanations (indicate pertinent contaminant)

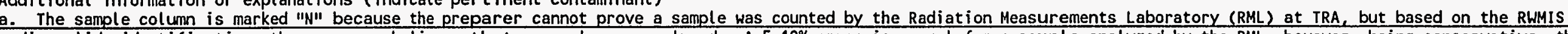

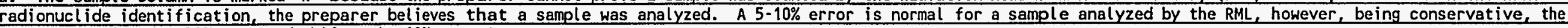
preparer assigned a $20 \%$ error (doubling the 10\% error).

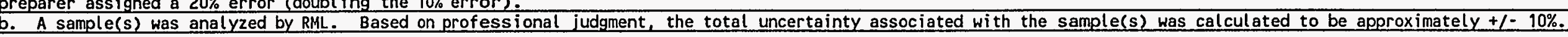


- each contaminant, complete at least one line on the following table. If any entries for that contaminant vary by year, fill out additional lines as needed

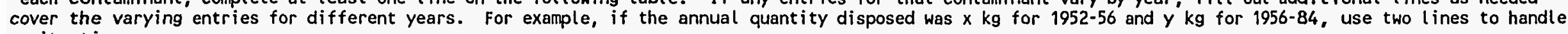
s situation.

\begin{tabular}{|c|c|c|c|c|c|c|c|c|c|c|}
\hline Radionuclide & Physical form & Chemical Form & $\begin{array}{l}\text { (A)nnual/(T)otal } \\
\text { Quantity }\end{array}$ & Unit & $\begin{array}{l}\text { Begin } \\
\text { Year }\end{array}$ & $\begin{array}{l}\text { End } \\
\text { Year }\end{array}$ & $\begin{array}{l}\text { Samp } \\
\text { les? }\end{array}$ & $\begin{array}{c}\text { Minimum } \\
\text { Value/\#Samp }\end{array}$ & $\begin{array}{r}\text { Maximum } \\
\text { Value/STD }\end{array}$ & $\begin{array}{l}\text { Basis for } \\
\text { Uncertainty }\end{array}$ \\
\hline Pu-238 & Resin. & Unknown. & T. .00445800000000 & CI & 1985 & 1985 & N & & & See cont inuation page. \\
\hline Pu-239 & Resin. & Unknown. & T. .00113900000000 & $\mathrm{Cl}$ & 1985 & 1985 & N & & & See continuation page. \\
\hline$P u-240$ & Resin. & Unknown. & T. .00069350000000 & $\mathrm{Cl}$ & 1985 & 1985 & N & & & See continuation page. \\
\hline$P u-241$ & Resin. & Unknown. & T. .37150000000000 & $\mathrm{CI}$ & 1985 & 1985 & N & & & See continuation page. \\
\hline$A m-241$ & Resin. & Unknown. & $T .09907000000000$ & CI & 1985 & 1985 & N & & & See continuation page. \\
\hline $\mathrm{Cm}-242$ & Resin. & Unknown. & $T .00693500000000$ & CI & 1985 & 1985 & N & & & See continuation page. \\
\hline $\mathrm{Cm}-244$ & Resin. & Unknown. & $T .00322000000000$ & $\mathrm{CI}$ & 1985 & 1985 & N & & & See continuation page. \\
\hline Ba-140 & Resin. & Unknown. & $\mathrm{T} .07800000000000$ & CI & 1985 & 1985 & N & $-20 \%$ & $+20 \%$ & See comment (a) below. \\
\hline $\mathrm{Ce}-141$ & Resin. & Unknown. & T. .38000000000000 & CI & 1985 & 1985 & N & $-20 \%$ & $+20 \%$ & See comment (a) below. \\
\hline
\end{tabular}

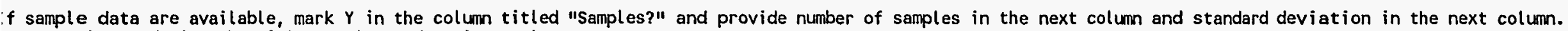

not, mark $N$ and give the minimum value and maximum value.

ditional information or explanations (indicate pertinent contaminant)

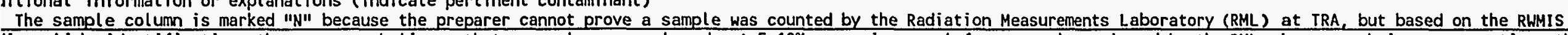

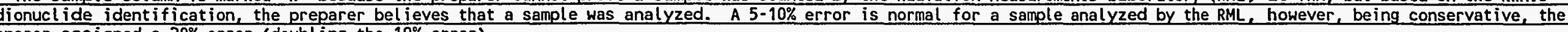
aparer ass igned a $20 \%$ error (doubling the $10 \%$ error).

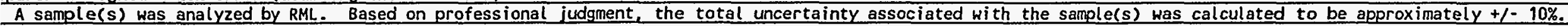


each contaminant, complete at least one line on the following table. If any entries for that contaminant vary by year, fill out additional lines as needed

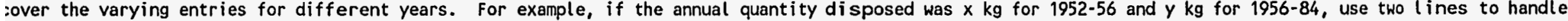
isituation.

\begin{tabular}{|c|c|c|c|c|c|c|c|c|c|c|}
\hline Radionuclide & Physical Form & Chemical form & $\begin{array}{l}\text { (A)nnual / ( T ) otal } \\
\text { Quant i ty }\end{array}$ & Unit & $\begin{array}{l}\text { Begin } \\
\text { Year }\end{array}$ & $\begin{array}{l}\text { End } \\
\text { Year }\end{array}$ & $\begin{array}{l}\text { Samp } \\
\text { les? }\end{array}$ & $\begin{array}{c}\text { Minimum } \\
\text { Value/HSamp }\end{array}$ & $\begin{array}{r}\text { Maximum } \\
\text { value/STD }\end{array}$ & $\begin{array}{l}\text { Basis for } \\
\text { Uncertainty }\end{array}$ \\
\hline $2 n-65$ & Resin. & Unknown. & T. .3264000000000 & CI & 1985 & 1985 & N & $-20 \%$ & $+20 \%$ & See comment (a) below. \\
\hline$H-3$ & Resin. & Unknown. & T. .00435500000000 & CI & 1986 & 1986 & N & & & See continuation page. \\
\hline$c-14$ & Resin. & Unknown. & T. .03746000000000 & CI & 1986 & 1986 & N & & & See continuation page. \\
\hline$F e-55$ & Resin. & Unknown. & T 1.742000000000 & CI & 1986 & 1986 & N & & & see continuation page. \\
\hline Ni-59 & Resin. & Unknown. & $T .02439000000000$ & $\mathrm{CI}$ & 1986 & 1986 & $\mathrm{~N}$ & & & See continuation page. \\
\hline $\mathrm{Ni}-63$ & Resin. & Unknown. & T 2.4390000000000 & $\mathrm{CI}$ & 1986 & 1986 & $N$ & & & See continuation page. \\
\hline $\mathrm{Co}-60$ & Resin. & Unknown. & T 5.9230000000000 & $\mathrm{CI}$ & 1986 & 1986 & N & & & See cont inuation page. \\
\hline sr-90 & Resin. & Unknown. & T 2.4390000000000 & CI & 1986 & 1986 & N & & & See continuation page. \\
\hline Tc-99 & Resin. & Unknown. & T.00013070000000 & CI & 1986 & 1986 & N & & & See continuation page. \\
\hline
\end{tabular}

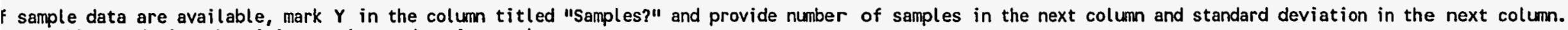
not, mark $N$ and give the minimum value and maximum value.

itional information or explanations (indicate pertinent contaminant)

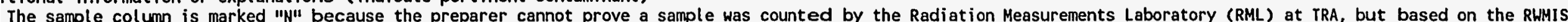

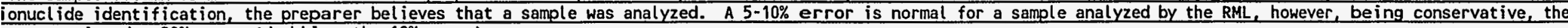
jarer assigned a $20 \%$ error (doubling the 10\% error). 
$r$ each contaminant, complete at least one line on the following table. If any entries for that contaminant vary by year, fill out additional lines as needed

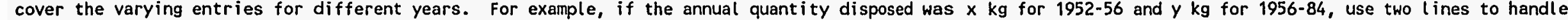
is situation

\begin{tabular}{|c|c|c|c|c|c|c|c|c|c|c|}
\hline Radionuclide & Physical form & Chemical Form & $\begin{array}{l}\text { (A)nnual } /(T) \text { otal } \\
\text { Quant i ty }\end{array}$ & Unit & $\begin{array}{l}\text { Begin } \\
\text { Year }\end{array}$ & $\begin{array}{l}\text { End } \\
\text { Year }\end{array}$ & $\begin{array}{l}\text { Samp } \\
\text { les? }\end{array}$ & $\begin{array}{c}\text { Minimum } \\
\text { Value/\#Samp }\end{array}$ & $\begin{array}{r}\text { Maximum } \\
\text { Value/STD }\end{array}$ & $\begin{array}{l}\text { Basis for } \\
\text { Uncertainty }\end{array}$ \\
\hline $\mathrm{Cm}-244$ & Resin. & Unknown. & T. .00097650000000 & CI & 1987 & 1987 & N & & & See continuation page. \\
\hline $\mathrm{Ag}-110$ & Resin. & Unknown. & T 1.9130000000000 & CI & 1987 & 1987 & N & $-20 \%$ & $+20 \%$ & See comment (a) below. \\
\hline $\mathrm{Ce}-144$ & Resin. & Unknown. & T 13.050000000000 & CI & 1987 & 1987 & N & $-20 \%$ & $+20 \%$ & See comment (a) below. \\
\hline Co-58 & Resin. & Unknown. & T 25.685900000000 & CI & 1987 & 1987 & N & $-20 \%$ & $+20 \%$ & See comment (a) below. \\
\hline $\mathrm{Co}-60$ & Resin. & Unknown. & $T 43.697230000000$ & CI & 1987 & 1987 & N & $-20 \%$ & $+20 \%$ & See comment (a) below. \\
\hline $\mathrm{Cr}-51$ & Resin. & Unknown. & T 261.85300000000 & CI & 1987 & 1987 & N & $-20 \%$ & $+20 \%$ & See comment (a) below. \\
\hline$H f-175$ & Resin. & Unknown. & T 2.7410000000000 & CI & 1987 & 1987 & N & $-20 \%$ & $+20 \%$ & See comment (a) below. \\
\hline$M n-54$ & Resin. & Unknown. & T 9.6755000000000 & CI & 1987 & 1987 & $N$ & $-20 \%$ & $+20 \%$ & See comment (a) below. \\
\hline
\end{tabular}

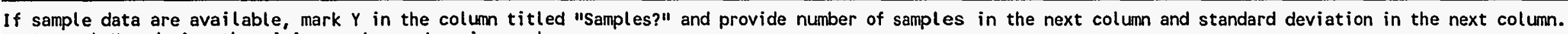
not, mark $N$ and give the minimum value and maximum value.

ditional information or explanations (indicate pertinent contaminant)

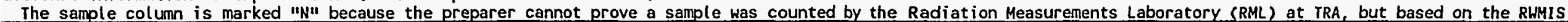

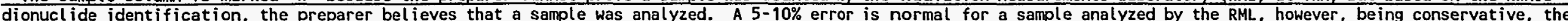

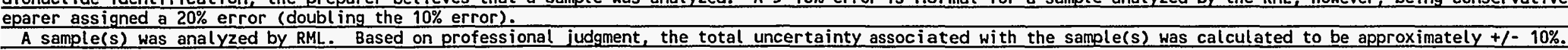




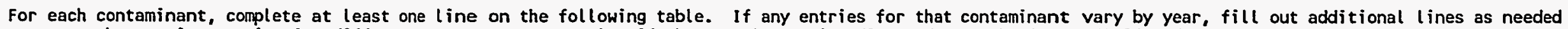

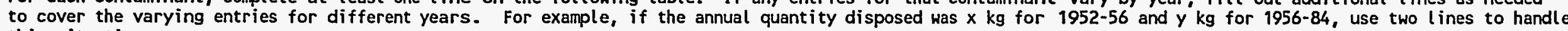
this situation.

\begin{tabular}{|c|c|c|c|c|c|c|c|c|c|c|}
\hline Radionuclide & Physical Form & Chemical Form & $\begin{array}{l}\text { (A)nnual/(T)otal } \\
\text { Quantity }\end{array}$ & Unit & $\begin{array}{l}\text { Begin } \\
\text { Year }\end{array}$ & $\begin{array}{l}\text { End } \\
\text { Year }\end{array}$ & $\begin{array}{l}\text { Samp } \\
\text { les? }\end{array}$ & $\begin{array}{c}\text { Minimum } \\
\text { Value/\#Samp }\end{array}$ & $\begin{array}{r}\text { Maximum } \\
\text { Value/STD }\end{array}$ & $\begin{array}{l}\text { Basis for } \\
\text { Uncertainty }\end{array}$ \\
\hline Pu-238 & Resin. & Unknown. & T. .00059720000000 & CI & 1988 & 1988 & $\mathbf{N}$ & & & See continuation page. \\
\hline Pu-239 & Resin. & Unknown. & T. .00015260000000 & CI & 1988 & 1988 & N & & & See continuation page. \\
\hline Pu-240 & Resin. & Unknown. & T. .00009289000000 & CI & 1988 & 1988 & N & & & See continuation page. \\
\hline Pu-241 & Resin. & Unknown. & T. .04976000000000 & CI & 1988 & 1988 & N & & & See continuation page. \\
\hline Am-241 & Resin. & Unknown. & $T .01327000000000$ & CI & 1988 & 1988 & N & & & See continuation page. \\
\hline $\mathrm{Cm}-242$ & Resin. & Unknown. & $T .00092890000000$ & $\mathrm{CI}$ & 1988 & 1988 & N & & & See continuation page. \\
\hline $\mathrm{Cm}-244$ & Resin. & Unknown. & $T .00043130000000$ & CI & 1988 & 1988 & N & & & See continuation page. \\
\hline $\mathrm{Ag}-110$ & Resin. & Unknown. & $T .02370000000000$ & $\mathrm{CI}$ & 1988 & 1988 & N & $-20 \%$ & $+20 \%$ & See comment (a) below. \\
\hline $\mathrm{Ce}-144$ & Resin. & Unknown. & $T .21600000000000$ & CI & 1988 & 1988 & N & $-20 \%$ & $+20 \%$ & See comment (a) below. \\
\hline
\end{tabular}

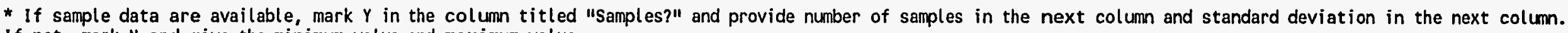
If not, mark $N$ and give the minimum value and maximum value.

Additional information or explanations (indicate pertinent contaminant)

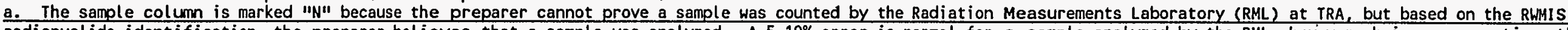

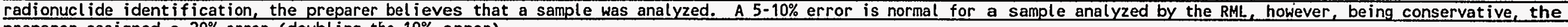
preparer assigned a $20 \%$ error (doubling the $10 \%$ error).

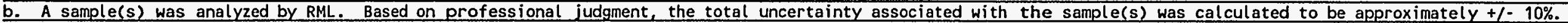


For each contaminant, complete at least one line on the following table. If any entries for that contaminant vary by year, fill out additional lines as needed to cover the varying entries for different years. For example, if the annual quantity disposed was $x \mathrm{~kg}$ for $1952-56$ and $y$ kg for 1956-84, use two lines to handle this situation.
th

\begin{tabular}{|c|c|c|c|c|c|c|c|c|c|c|}
\hline Radionucl ide & Physical Form & Chemical Form & $\begin{array}{l}\text { (A)nnual/(T)otal } \\
\text { Quantity }\end{array}$ & Unit & $\begin{array}{l}\text { Begin } \\
\text { Year }\end{array}$ & $\begin{array}{l}\text { End } \\
\text { Year }\end{array}$ & $\begin{array}{l}\text { Samp } \\
\text { les? }\end{array}$ & $\begin{array}{c}\text { Minimum } \\
\text { Value/\#Samp }\end{array}$ & $\begin{array}{r}\text { Maximum } \\
\text { Value/STD }\end{array}$ & $\begin{array}{l}\text { Basis for } \\
\text { Uncertainty }\end{array}$ \\
\hline Pu-239 & Resin. & Unknown. & T. .00005347000000 & $\mathrm{Cl}$ & 1989 & 1989 & N & & & See continuation page. \\
\hline Pu-240 & Resin. & Unknown. & $T .00003255000000$ & Cl & 1989 & 1989 & N & & & See continuation page. \\
\hline Pu-241 & Resin. & Unknown. & $T .01744000000000$ & CI & 1989 & 1989 & N & & & See continuation page. \\
\hline Am-241 & Resin. & Unknown. & T. .00465000000000 & CI & 1989 & 1989 & N & & & See continuation page. \\
\hline $\mathrm{Cm}-242$ & Resin. & Unknown. & T. .00032550000000 & CI & 1989 & 1989 & N & & & See continuation page. \\
\hline $\mathrm{Cm}-244$ & Resin. & Unknown. & T. .00015110000000 & CI & 1989 & 1989 & N & & & See continuation page. \\
\hline $\mathrm{Ce}-144$ & Resin. & Unknown. & T. .15200000000000 & CI & 1989 & 1989 & N & $-20 \%$ & $+20 \%$ & See comment (a) below. \\
\hline Co-58 & Resin. & Unknown. & T. .73800000000000 & CI & 1989 & 1989 & N & $-20 \%$ & $+20 \%$ & See comment (a) below. \\
\hline Co- 60 & Resin. & Unknown. & T 2.9070000000000 & CI & 1989 & 1989 & N & $-20 \%$ & $+20 \%$ & See comment (a) below. \\
\hline
\end{tabular}

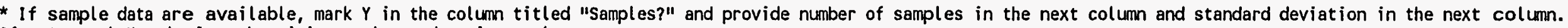
If not, mark $N$ and give the minimum value and maximum value.

Additional information or explanations (indicate pertinent contaminant)

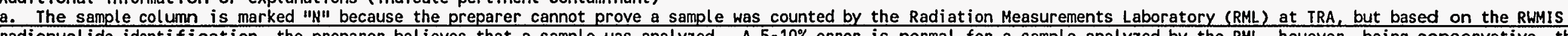

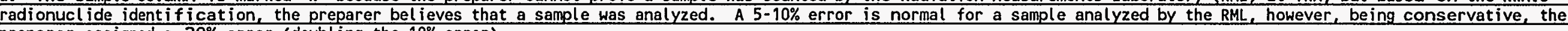
preparer assigned a $20 \%$ error (doubling the $10 \%$ error).

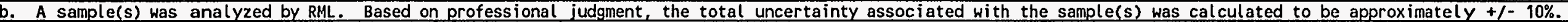


Ir each contaminant, complete at least one line on the following table. If any entries for that contaminant vary by year, fill out additional lines as needed

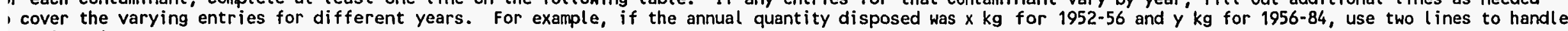
is situation.

\begin{tabular}{|c|c|c|c|c|c|c|c|c|c|c|}
\hline Radionuclide & Physical Form & Chemical form & $\begin{array}{l}\text { (A)nnual/( } T \text { ) otal } \\
\text { Quant ity }\end{array}$ & Unit & $\begin{array}{l}\text { Begin } \\
\text { Year }\end{array}$ & $\begin{array}{l}\text { End } \\
\text { Year }\end{array}$ & $\begin{array}{l}\text { Samp } \\
\text { les? }\end{array}$ & $\begin{array}{c}\text { Minimum } \\
\text { Value/\#Samp }\end{array}$ & $\begin{array}{r}\text { Maximum } \\
\text { Value/STD }\end{array}$ & $\begin{array}{l}\text { Basis for } \\
\text { Uncertainty }\end{array}$ \\
\hline $\mathrm{Cr}-51$ & Resin. & Unknown. & 厂 71.775000000000 & CI & 1989 & 1989 & N & $-20 \%$ & $+20 \%$ & See comment (a) below. \\
\hline$H f-181$ & Resin. & Unknown. & T. .14730000000000 & $\mathrm{Cl}$ & 1989 & 1989 & N & $-20 \%$ & $+20 \%$ & See comment (a) below. \\
\hline$M n-54$ & Res in. & Unknown. & T. .10100000000000 & $\mathrm{Cl}$ & 1989 & 1989 & N & $-20 \%$ & $+20 \%$ & See comment (a) below. \\
\hline $2 n-65$ & Resin. & Unknown. & $T .34570000000000$ & $\mathrm{CI}$ & 1989 & 1989 & N & $-20 \%$ & $+20 \%$ & See comment (a) below. \\
\hline $\mathrm{H}-3$ & Resin. & Unknown. & $T .00018240000000$ & $\mathrm{CI}$ & 1990 & 1990 & N & & & See continuation page. \\
\hline$c-14$ & Resin. & Unknown. & T. .00156900000000 & CI & 1990 & 1990 & $\mathbf{N}$ & & & See continuation page. \\
\hline $\mathrm{Fe}-55$ & Resin. & Unknown. & T.07296000000000 & $\mathrm{CI}$ & 1990 & 1990 & N & & & See continuation page. \\
\hline $\mathrm{Ni}-59$ & Resin. & Unknown. & $T .00102100000000$ & $\mathrm{CI}$ & 1990 & 1990 & N & & & See continuation page. \\
\hline $\mathrm{Ni}-63$ & Resin. & Unknown. & $T .10210000000000$ & $\mathrm{Cl}$ & 1990 & 1990 & N & & & See continuation page. \\
\hline
\end{tabular}

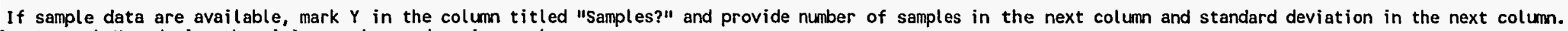

not, mark $N$ and give the minimum value and maximum value.

Iditional information or explanations (indicate pertinent contaminant)

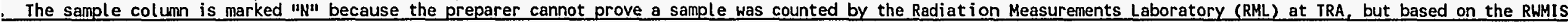

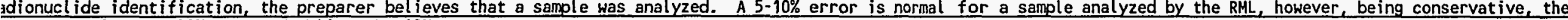
eparer assigned a $20 \%$ error (doubl ing the 10\% error)

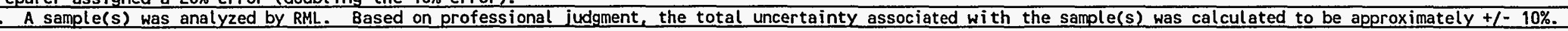




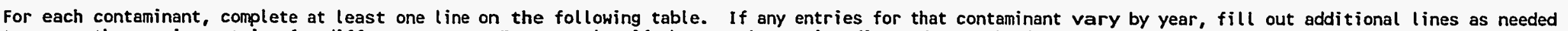

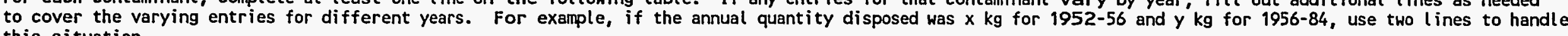
this situation.

\begin{tabular}{|c|c|c|c|c|c|c|c|c|c|c|}
\hline Radionuclide & Physical Form & Chemical form & $\begin{array}{l}\text { (A)nnual/(T)otal } \\
\text { Quantity }\end{array}$ & Unit & $\begin{array}{l}\text { Begin } \\
\text { Year }\end{array}$ & $\begin{array}{l}\text { End } \\
\text { Year }\end{array}$ & $\begin{array}{l}\text { Samp } \\
\text { les? }\end{array}$ & $\begin{array}{c}\text { Minimum } \\
\text { Value/\#Samp }\end{array}$ & $\begin{array}{c}\text { Maximum } \\
\text { Value/STD }\end{array}$ & $\begin{array}{l}\text { Basis for } \\
\text { Uncertainty }\end{array}$ \\
\hline Co- 60 & Resin. & Unknown. & $T .24810000000000$ & $\mathrm{CI}$ & 1990 & 1990 & N & & & See continuation page. \\
\hline Sr-90 & Resin. & Unknown. & $T .10210000000000$ & CI & 1990 & 1990 & N & & & See continuation page. \\
\hline Tc-99 & Resin. & Unknown. & $T .00000547200000$ & $\mathrm{CI}$ & 1990 & 1990 & N & & & See continuation page. \\
\hline$I-129$ & Resin. & Unknown. & $T .00000002262000$ & $\mathrm{CI}$ & 1990 & 1990 & N & & & See continuation page. \\
\hline Cs -137 & Resin. & Unknown. & T. .11310000000000 & $\mathrm{CI}$ & 1990 & 1990 & $\mathbf{N}$ & & & See continuation page. \\
\hline $\mathrm{Ce}-144$ & Resin. & Unknown. & $T .00280900000000$ & $\mathrm{Cl}$ & 1990 & 1990 & N & & & See continuation page. \\
\hline Eu- 154 & Resin. & Unknown. & T. .00266300000000 & CI & 1990 & 1990 & N & & & See cont inuation page. \\
\hline Eu- 155 & Resin. & Unknown. & T. .00113100000000 & $\mathrm{CI}$ & 1990 & 1990 & N & & & See continuation page. \\
\hline$U-234$ & Resin. & Unknown. & $T .00000153200000$ & $\mathrm{CI}$ & 1990 & 1990 & N & & & See continuation page. \\
\hline
\end{tabular}

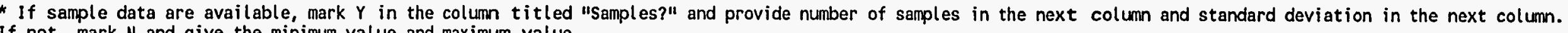
If not, mark $N$ and give the minimum value and maximum value.

Additional information or explanations (indicate pertinent contaminant)

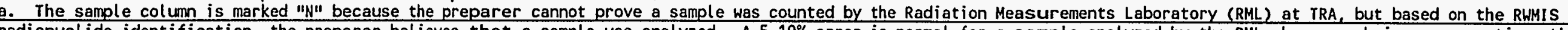

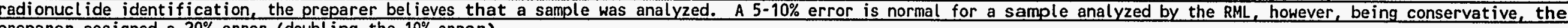
oreparer assigned a $20 \%$ error (doubling the $10 \%$ error).

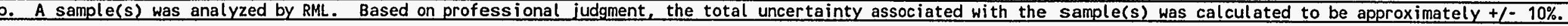


each contaminant, complete at least one line on the following table. If any entries for that contaminant vary by year, fill out additional lines as needed

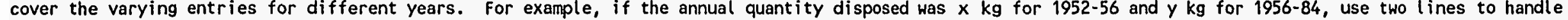
s situation.

\begin{tabular}{|c|c|c|c|c|c|c|c|c|c|c|}
\hline Radionucl ide & Physical form & Chemical Form & $\begin{array}{l}\text { (A)nnual / ( T )otal } \\
\text { Quantity }\end{array}$ & Unit & $\begin{array}{l}\text { Begin } \\
\text { Year }\end{array}$ & $\begin{array}{l}\text { End } \\
\text { Year }\end{array}$ & $\begin{array}{l}\text { Samp } \\
\text { les? }\end{array}$ & $\begin{array}{c}\text { Minimum } \\
\text { value/\#Samp }\end{array}$ & $\begin{array}{r}\text { Maximum } \\
\text { Value/STD }\end{array}$ & $\begin{array}{l}\text { Basis for } \\
\text { Uncertainty }\end{array}$ \\
\hline Cs -137 & Resin. & Unknown. & T.14725000000000 & CI & 1990 & 1990 & N & $-20 \%$ & $+20 \%$ & See comment (a) below. \\
\hline Eu-152 & Resin. & Unknown. & $T .00160000000000$ & CI & 1990 & 1990 & N & $-20 \%$ & $+20 \%$ & See comment (a) below. \\
\hline$H-3$ & Resin. & Unknown. & T.00782000000000 & $\mathrm{CI}$ & 1990 & 1990 & $N$ & $-20 \%$ & $+20 \%$ & See comment (a) below. \\
\hline$H f-181$ & Resin. & Unknown. & T. .33950000000000 & CI & 1990 & 1990 & N & $-20 \%$ & $+20 \%$ & See comment (a) below. \\
\hline La-140 & Resin. & Unknown. & T. .10045000000000 & CI & 1990 & 1990 & N & $-20 \%$ & $+20 \%$ & See comment (a) below. \\
\hline$M n-54$ & Resin. & Unknown. & T. .66196000000000 & $\mathrm{Cl}$ & 1990 & 1990 & $N$ & $-20 \%$ & $+20 \%$ & See comment (a) below. \\
\hline $\mathrm{Nb}-95$ & Resin. & Unknown. & T. .37118000000000 & CI & 1990 & 1990 & N & $-20 \%$ & $+20 \%$ & See comment (a) below. \\
\hline $\mathrm{Ni}-63$ & Resin. & Unknown. & $r .15400000000000$ & CI & 1990 & 1990 & $\mathbf{N}$ & $-20 \%$ & $+20 \%$ & See comment (a) below. \\
\hline
\end{tabular}

if sample data are available, mark $Y$ in the column titled "Samples?" and provide number of samples in the next column and standard deviation in the next column. not, mark $N$ and give the minimum value and maximum value.

ditional information or explanations (indicate pertinent contaminant)

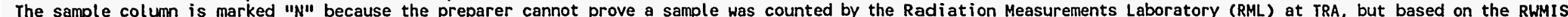

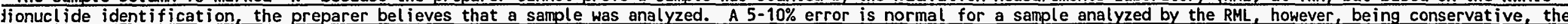
jorer assigned a $20 \%$ error (doubling the $10 \%$ error).

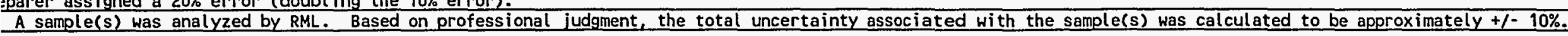


each contaminant, complete at least one line on the following table. If any entries for that contaminant vary by year, fill out additional lines as needed

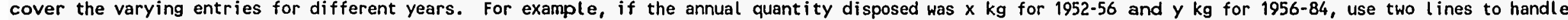
s situation.

\begin{tabular}{|c|c|c|c|c|c|c|c|c|c|c|}
\hline Radionuclide & Physical Form & Chemical Form & $\begin{array}{l}\text { (A)nnual/(T)otal } \\
\text { Quantity }\end{array}$ & Unit & $\begin{array}{l}\text { Begin } \\
\text { Year }\end{array}$ & $\begin{array}{l}\text { End } \\
\text { Year }\end{array}$ & $\begin{array}{l}\text { Samp } \\
\text { les? }\end{array}$ & $\begin{array}{c}\text { Minimum } \\
\text { Value/\#Samp }\end{array}$ & $\begin{array}{r}\text { Maximum } \\
\text { Value/STD }\end{array}$ & $\begin{array}{l}\text { Basis for } \\
\text { Uncertainty }\end{array}$ \\
\hline sr-92 & Resin. & Unknown. & $T .00160000000000$ & $\mathrm{CI}$ & 1990 & 1990 & N & $-20 \%$ & $+20 \%$ & See comment (a) below. \\
\hline Ta-182 & Resin. & Unknown. & T. .00605000000000 & $\mathrm{CI}$ & 1990 & 1990 & N & $-20 \%$ & $+20 \%$ & See comment (a) below. \\
\hline$Y-88$ & Resin. & Unknown. & T. .00300000000000 & $\mathrm{CI}$ & 1990 & 1990 & $\mathbf{N}$ & $-20 \%$ & $+20 \%$ & See coment (a) below. \\
\hline $2 n-65$ & Resin. & Unknown. & $T 1.2113000000000$ & CI & 1990 & 1990 & N & $-20 \%$ & $+20 \%$ & See comment (a) below. \\
\hline $2 r-95$ & Resin. & Unknown. & $T .33160000000000$ & $\mathrm{Cl}$ & 1990 & 1990 & N & $-20 \%$ & $+20 \%$ & See comment (a) below. \\
\hline$B a-140$ & Resin. & Unknown. & $T .06060000000000$ & CI & 1991 & 1991 & N & $-20 \%$ & $+20 \%$ & See comment (a) below. \\
\hline$B r-82$ & Resin. & Unknown. & $T .00100000000000$ & CI & 1991 & 1991 & $\mathbf{N}$ & $-20 \%$ & $+20 \%$ & See comment (a) below. \\
\hline$C d-109$ & Resin. & Unknown. & $T .01090000000000$ & $\mathrm{Cl}$ & 1991 & 1991 & $\mathbf{N}$ & $-20 \%$ & $+20 \%$ & See comment (a) below. \\
\hline $\mathrm{Ce}-141$ & Resin. & Unknown. & $\tau .17060000000000$ & $\mathrm{Cl}$ & 1991 & 1991 & $\mathbf{N}$ & $-20 \%$ & $+20 \%$ & See comment (a) below. \\
\hline
\end{tabular}

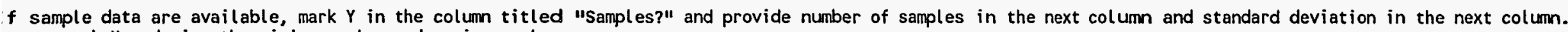
not, mark $N$ and give the minimum value and maximum value.

ditional information or explanations (indicate pertinent contaminant)

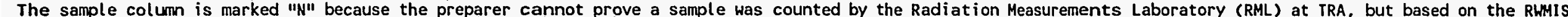

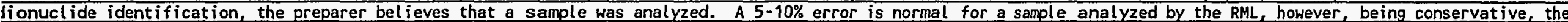
parer assigned a $20 \%$ error (doubling the $10 \%$ error). 
each contaminant, complete at least one line on the following table. If any entries for that contaminant vary by year, fill out additional lines as needed

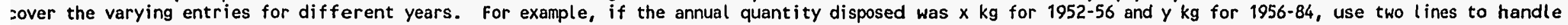
s situation.

\begin{tabular}{|c|c|c|c|c|c|c|c|c|c|c|}
\hline Radionuclide & Physical Form & Chemical form & $\begin{array}{l}\text { (A)nnual/(T)otal } \\
\text { Quantity }\end{array}$ & Unit & $\begin{array}{l}\text { Begin } \\
\text { Year }\end{array}$ & $\begin{array}{l}\text { End } \\
\text { Year }\end{array}$ & $\begin{array}{l}\text { Samp } \\
\text { les? }\end{array}$ & $\begin{array}{c}\text { Minimum } \\
\text { Value/\#Samp }\end{array}$ & $\begin{array}{r}\text { Maximum } \\
\text { Value/STD }\end{array}$ & $\begin{array}{l}\text { Basis for } \\
\text { Uncertainty }\end{array}$ \\
\hline $\mathrm{Fe}-59$ & Resin. & Unknown. & $T .00200000000000$ & $\mathrm{CI}$ & 1991 & 1991 & N & $-20 \%$ & $+20 \%$ & See comment (a) below. \\
\hline$H-3$ & Resin. & Unknown. & $T .02820000000000$ & $\mathrm{CI}$ & 1991 & 1991 & N & $-20 \%$ & $+20 \%$ & See comment (a) below. \\
\hline$H f-175$ & Resin. & Unknown. & $r .04090000000000$ & CI & 1991 & 1991 & N & $-20 \%$ & $+20 \%$ & See comment (a) below. \\
\hline$H f-181$ & Resin. & Unknown. & $T .14100000000000$ & CI & 1991 & 1991 & N & $-20 \%$ & $+20 \%$ & See comment (a) below. \\
\hline $1-131$ & Resin. & Unknown. & $T .05680000000000$ & Cl & 1991 & 1991 & N & $-20 \%$ & $+20 \%$ & See comment (a) below. \\
\hline $1-132$ & Resin. & Unknown. & $T .04460000000000$ & CI & 1991 & 1991 & N & $-20 \%$ & $+20 \%$ & See comment (a) below. \\
\hline$L a-140$ & Resin. & Unknown. & T. .07750000000000 & CI & 1991 & 1991 & N & $-20 \%$ & $+20 \%$ & See comment (a) below. \\
\hline$M n-54$ & Resin. & Unknown. & T. .49750000000000 & CI & 1991 & 1991 & N & $-20 \%$ & $+20 \%$ & See corment (a) below. \\
\hline $\mathrm{Nb}-95$ & Resin. & Unknown. & $T .10930000000000$ & CI & 1991 & 1991 & N & $-20 \%$ & $+20 \%$ & See comment (a) below. \\
\hline
\end{tabular}

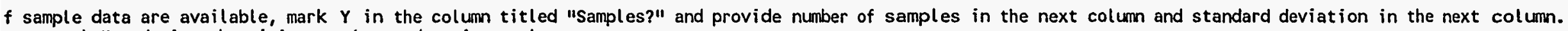
not, mark $N$ and give the minimum value and maximum value.

litional information or explanations (indicate pertinent contaminant)

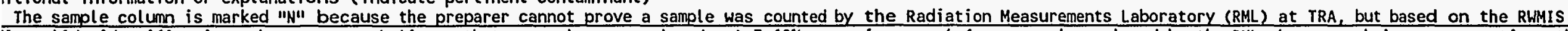

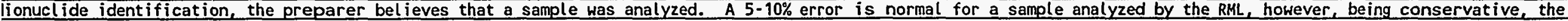
parer assigned a $20 \%$ error (doubl ing the $10 \%$ error)

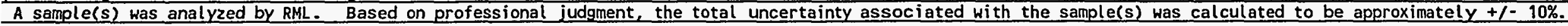




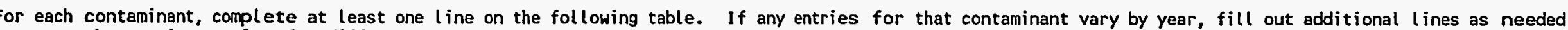

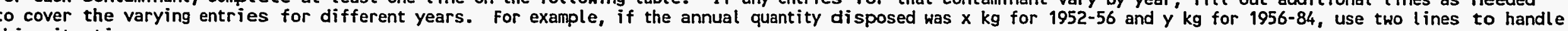
his situation.

\begin{tabular}{|c|c|c|c|c|c|c|c|c|c|c|}
\hline Radionucl ide & Physical Form & Chemical form & $\begin{array}{l}\text { (A)nnual / ( T ) otal } \\
\text { Quant ity }\end{array}$ & Unit & $\begin{array}{l}\text { Begin } \\
\text { Year }\end{array}$ & $\begin{array}{l}\text { End } \\
\text { Year }\end{array}$ & $\begin{array}{l}\text { Samp } \\
\text { les? }\end{array}$ & $\begin{array}{c}\text { Minimum } \\
\text { Value/\#Samp }\end{array}$ & $\begin{array}{r}\text { Maximum } \\
\text { Value/STO }\end{array}$ & $\begin{array}{l}\text { Basis for } \\
\text { Uncertainty }\end{array}$ \\
\hline $\operatorname{Pr}-144$ & Resin. & Unknown. & T. .09500000000000 & CI & 1991 & 1991 & N & $-20 \%$ & $+20 \%$ & See comment (a) below. \\
\hline Ru-103 & Resin. & Unknown. & T. .01890000000000 & $\mathrm{CI}$ & 1991 & 1991 & N & $-20 \%$ & $+20 \%$ & See comment (a) below. \\
\hline Sb-124 & Resin. & Unknown. & T. .00540000000000 & $\mathrm{CI}$ & 1991 & 1991 & N & $-20 \%$ & $+20 \%$ & See comment (a) below. \\
\hline Sc-46 & Resin. & Unknown. & T. .02990000000000 & CI & 1991 & 1991 & N & $-20 \%$ & $+20 \%$ & See comment (a) below. \\
\hline $\mathrm{Se}-75$ & Resin. & Unknown. & $T .02000000000000$ & CI & 1991 & 1991 & N & $-20 \%$ & $+20 \%$ & See comment (a) below. \\
\hline $5 r-89$ & Resin. & Unknown. & T. .00090000000000 & CI & 1991 & 1991 & N & $-20 \%$ & $+20 \%$ & See comment (a) below. \\
\hline $5 r-90$ & Resin. & Unknown. & T. .35840000000000 & CI & 1991 & 1991 & N & $-20 \%$ & $+20 \%$ & See comment (a) below. \\
\hline Ta-182 & Resin. & Unknown. & T. .00160000000000 & $\mathrm{Cl}$ & 1991 & 1991 & N & $-20 \%$ & $+20 \%$ & See comment (a) below. \\
\hline $2 n-65$ & Resin. & Unknown. & T 2.1672000000000 & CI & 1991 & 1991 & N & $-20 \%$ & $+20 \%$ & See comment (a) below. \\
\hline
\end{tabular}

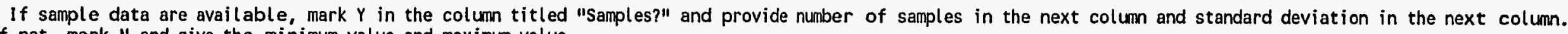

not, mark $N$ and give the minimum value and maximum value.
dditional information or explanations (indicate pertinent contaminant)

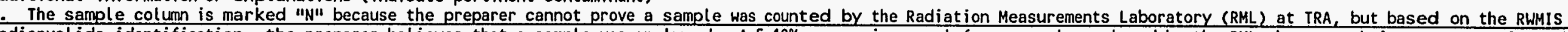

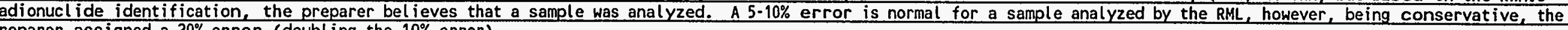
reparer assigned a $20 \%$ error (doubling the $10 \%$ error).

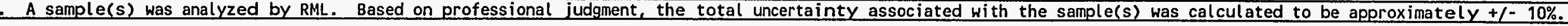




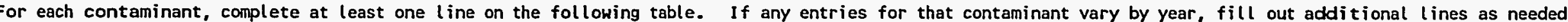

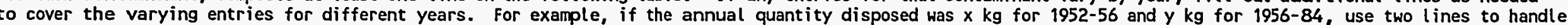
this situation.

\begin{tabular}{|c|c|c|c|c|c|c|c|c|c|c|}
\hline Radionucl ide & Physical Form & Chemical Form & $\begin{array}{l}\text { (A)nnual/(T)otal } \\
\text { Quantity }\end{array}$ & Unit & $\begin{array}{l}\text { Begin } \\
\text { Year }\end{array}$ & $\begin{array}{l}\text { End } \\
\text { Year }\end{array}$ & $\begin{array}{l}\text { Samp } \\
\text { les? }\end{array}$ & $\begin{array}{c}\text { Minimum } \\
\text { Value/\#Samp }\end{array}$ & $\begin{array}{c}\text { Maximum } \\
\text { Value/STD }\end{array}$ & $\begin{array}{l}\text { Basis for } \\
\text { Uncertainty }\end{array}$ \\
\hline $\operatorname{Pr}-144$ & Resin. & Unknown. & $\mathrm{T} .06380000000000$ & CI & 1992 & 1992 & N & $-20 \%$ & $+20 \%$ & See comment (a) below. \\
\hline Re-188 & Resin. & Unknown. & T.00930000000000 & CI & 1992 & 1992 & N & $-20 \%$ & $+20 \%$ & See comment (a) below. \\
\hline Ru- 103 & Resin. & Unknown. & $T .06930000000000$ & $\mathrm{CI}$ & 1992 & 1992 & $" N$ & $-20 \%$ & $+20 \%$ & See comment (a) below. \\
\hline Sb- 124 & Resin. & Unknown. & $T .00030000000000$ & CI & 1992 & 1992 & N & $-20 \%$ & $+20 \%$ & See comment (a) below. \\
\hline Sc-46 & Resin. & Unknown. & $T .04050000000000$ & $\mathrm{CI}$ & 1992 & 1992 & N & $-20 \%$ & $+20 \%$ & See comment (a) below. \\
\hline Se-75 & Resin. & Unknown. & $T .00630000000000$ & $\mathrm{CI}$ & 1992 & 1992 & N & $-20 \%$ & $+20 \%$ & See comment (a) below. \\
\hline $5 r-89$ & Resin. & Unknown. & T 1.6750000000000 & CI & 1992 & 1992 & $N$ & $-20 \%$ & $+20 \%$ & See comment (a) below. \\
\hline $\mathrm{Sr}-90$ & Resin. & Unknown. & $T .78300000000000$ & $\mathrm{Cl}$ & 1992 & 1992 & N & $-20 \%$ & $+20 \%$ & See comment (a) below. \\
\hline Sr-91 & Resin. & Unknown. & $T .00440000000000$ & CI & 1992 & 1992 & N & $-20 \%$ & $+20 \%$ & See comment (a) below. \\
\hline
\end{tabular}

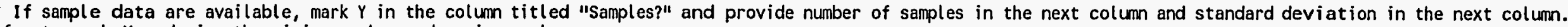
$f$ not, mark $N$ and give the minimum value and maximum value.

dditional information or explanations (indicate pertinent contaminant)

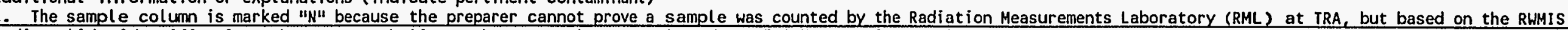

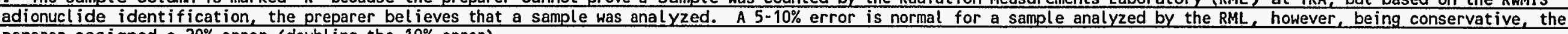
reparer assigned a 20\% error (doubl ing the $10 \%$ error).

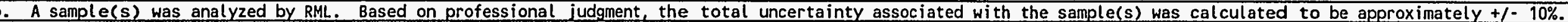


- each contaminant, complete at least one line on the following table. If any entries for that contaminant vary by year, fill out additional lines as needed

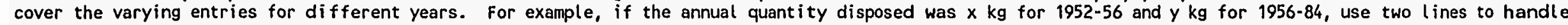
is situation.

\begin{tabular}{|c|c|c|c|c|c|c|c|c|c|c|}
\hline Radionuclide & Physical Form & Chemical Form & $\begin{array}{l}\text { (A)nnual } /(T) \text { otal } \\
\text { Quantity }\end{array}$ & Unit & $\begin{array}{l}\text { Begin } \\
\text { Year }\end{array}$ & $\begin{array}{l}\text { End } \\
\text { Year }\end{array}$ & $\begin{array}{l}\text { Samp } \\
\text { les? }\end{array}$ & $\begin{array}{c}\text { Minimum } \\
\text { Value/\#Samp }\end{array}$ & $\begin{array}{c}\text { Maximum } \\
\text { value/STD }\end{array}$ & $\begin{array}{l}\text { Basis for } \\
\text { Uncertainty }\end{array}$ \\
\hline Te-132 & Resin. & Unknown. & $T .00030000000000$ & $\mathrm{Cl}$ & 1992 & 1992 & $2 \mathrm{~N}$ & $-20 \%$ & $+20 \%$ & See corment (a) below. \\
\hline$Y-93$ & Resin. & Unknown. & T.11070000000000 & CI & 1992 & 1992 & N & $-20 \%$ & $+20 \%$ & See comment (a) below. \\
\hline $2 n-65$ & Resin. & Unknown. & T. .12862000000000 & CI & 1992 & 1992 & N & $-20 \%$ & $+20 \%$ & See comment (a) below. \\
\hline $2 r-95$ & Resin. & Unknown. & T. .18110000000000 & CI & 1992 & 1992 & N & $-20 \%$ & $+20 \%$ & See comment (a) below. \\
\hline Am-241 & Resin. & Unknown. & $T .01490000000000$ & $\mathrm{Cl}$ & 1993 & 1993 & N & $-20 \%$ & $+20 \%$ & See comment (a) below. \\
\hline$B a-140$ & Resin. & Unknown. & $T .00360000000000$ & CI & 1993 & 1993 & N & $-20 \%$ & $+20 \%$ & See comment (a) below. \\
\hline Ce-141 & Resin. & Unknown. & $T .03520000000000$ & CI & 1993 & 1993 & N & $-20 \%$ & $+20 \%$ & See comment (a) below. \\
\hline Ce-144 & Resin. & Unknown. & T. .57970000000000 & CI & 1993 & 1993 & N & $-20 \%$ & $+20 \%$ & See comment (a) below. \\
\hline Co-58 & Resin. & Unknown. & T. .22310000000000 & CI & 1993 & 1993 & N & $-20 \%$ & $+20 \%$ & See comment (a) below. \\
\hline
\end{tabular}

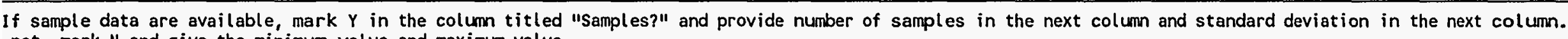
not, mark $N$ and give the minimum value and maximum value.

ditional information or explanations (indicate pertinent contaminant)

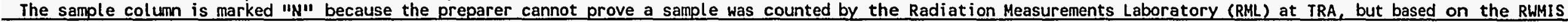

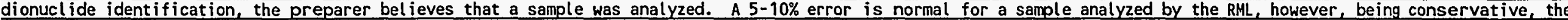
eparer assigned a $20 \%$ error (doubling the 10\% error).

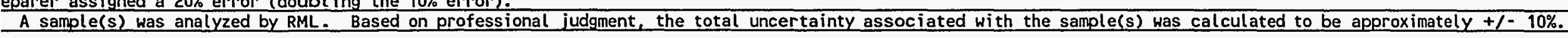




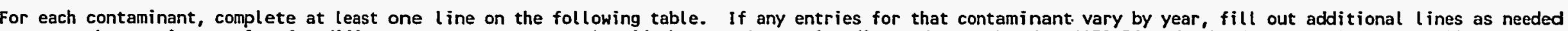

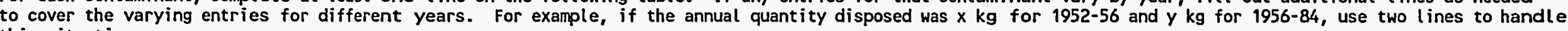
this situation.

\begin{tabular}{|c|c|c|c|c|c|c|c|c|c|c|}
\hline Radionucl ide & Physical Form & Chemical form & $\begin{array}{l}\text { (A)nnual/(T)otal } \\
\text { Quantity }\end{array}$ & Unit & $\begin{array}{l}\text { Begin } \\
\text { Year }\end{array}$ & $\begin{array}{l}\text { End } \\
\text { Year }\end{array}$ & $\begin{array}{l}\text { Samp } \\
\text { les? }\end{array}$ & $\begin{array}{c}\text { Minimum } \\
\text { Value/\#Samp }\end{array}$ & $\begin{array}{c}\text { Maximum } \\
\text { Value/STD }\end{array}$ & $\begin{array}{l}\text { Basis for } \\
\text { Uncertainty }\end{array}$ \\
\hline Co-60 & Resin. & Unknown. & T 2.8767700000000 & CI & 1993 & 1993 & N & $-10 \%$ & $+10 \%$ & See corment (b) below. \\
\hline $\mathrm{Cr}-51$ & Resin. & Unknown. & T 46.715600000000 & CI & 1993 & 1993 & N & $-20 \%$ & $+20 \%$ & See comment (a) below. \\
\hline Cs-134 & Resin. & Unknown. & T. .10599000000000 & $\mathrm{CI}$ & 1993 & 1993 & N & $-20 \%$ & $+20 \%$ & See comment (a) below. \\
\hline Cs-137 & Resin. & Unknown. & T 1.4748000000000 & CI & 1993 & 1993 & N & $-20 \%$ & $+20 \%$ & See comment (a) below. \\
\hline Eu-154 & Resin. & Unknown. & $T .01760000000000$ & CI & 1993 & 1993 & N & $-10 \%$ & $+10 \%$ & See comment (b) below. \\
\hline$H-3$ & Resin. & Unknown. & $\mathrm{T} .01360000000000$ & CI & 1993 & 1993 & N & $-10 \%$ & $+10 \%$ & See comment (b) below. \\
\hline$H f-181$ & Resin. & Unknown. & T. .03480000000000 & CI & 1993 & 1993 & N & $-10 \%$ & $+10 \%$ & See comment (b) below. \\
\hline $1-131$ & Resin. & Unknown. & $T .00660000000000$ & CI & 1993 & 1993 & N & $-10 \%$ & $+10 \%$ & See comment (b) below. \\
\hline Ir-192 & Resin. & Unknown. & T.00190000000000 & CI & 1993 & 1993 & N & $-20 \%$ & $+20 \%$ & See comment (a) below. \\
\hline
\end{tabular}

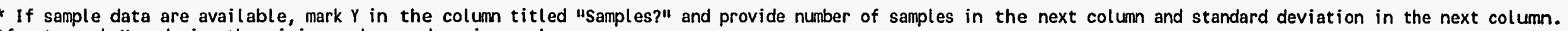
if not, mark $N$ and give the minimum value and maximum value.

idditional information or explanations (indicate pertinent contaminant)

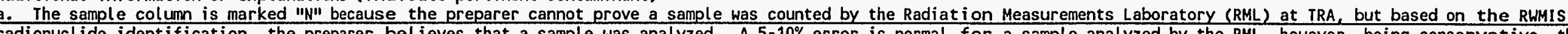

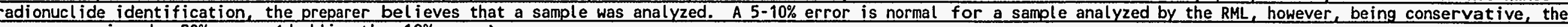
reparer assigned a $20 \%$ error (doubling the 10\% error).

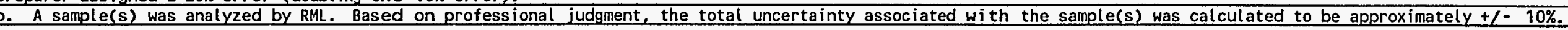


$r$ each contaminant, complete at least one line on the following table. If any entries for that contaminant vary by year, fill out additional lines as needed

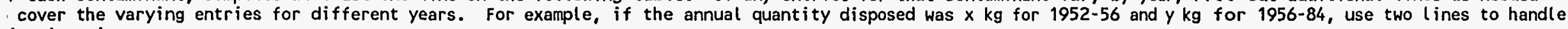
is situation

\begin{tabular}{|c|c|c|c|c|c|c|c|c|c|c|}
\hline Radionuclide & Physical Form & Chemical form & $\begin{array}{l}\text { (A)nnual/(T)otal } \\
\text { Quantity }\end{array}$ & Unit & $\begin{array}{l}\text { Begin } \\
\text { Year }\end{array}$ & $\begin{array}{l}\text { End } \\
\text { Year }\end{array}$ & $\begin{array}{l}\text { Samp } \\
\text { les? }\end{array}$ & $\begin{array}{c}\text { Minimum } \\
\text { value/\#Samp }\end{array}$ & $\begin{array}{r}\text { Maximum } \\
\text { Value/STO }\end{array}$ & $\begin{array}{l}\text { Basis for } \\
\text { Uncertainty }\end{array}$ \\
\hline La-140 & Resin. & Unknown. & T. .00360000000000 & CI & 1993 & 1993 & N & $-20 \%$ & $+20 \%$ & See comment (a) below. \\
\hline$M n-54$ & Resin. & Unknown. & $T .16600000000000$ & CI & 1993 & 1993 & N & $-20 \%$ & $+20 \%$ & See comment (a) below. \\
\hline Ho-99 & Resin. & Unknown. & T.01900000000000 & $\mathrm{CI}$ & 1993 & 1993 & N & $-20 \%$ & $+20 \%$ & See comment (a) below. \\
\hline Nb-95 & Resin. & Unknown. & $T .15210000000000$ & $\mathrm{CI}$ & 1993 & 1993 & N & $-20 \%$ & $+20 \%$ & See comment (a) below. \\
\hline $\mathrm{Pr}-144$ & Resin. & Unknown. & T.25370000000000 & CI & 1993 & 1993 & N & $-20 \%$ & $+20 \%$ & See comment (a) below. \\
\hline Ru- 103 & Resin. & Unknown. & T. .00560000000000 & CI & 1993 & 1993 & N & $-20 \%$ & $+20 \%$ & See comment (a) below. \\
\hline Sb-124 & Resin. & Unknown. & $T .00050000000000$ & CI & 1993 & 1993 & N & $-20 \%$ & $+20 \%$ & See comment (a) below. \\
\hline Sb- 125 & Resin. & Unknown. & $T .00400000000000$ & CI & 1993 & 1993 & N & $-20 \%$ & $+20 \%$ & See comment (a) below. \\
\hline Sc-46 & Resin. & Unknown. & T. .00560000000000 & CI & 1993 & 1993 & N & $-20 \%$ & $+20 \%$ & See comment (a) below. \\
\hline
\end{tabular}

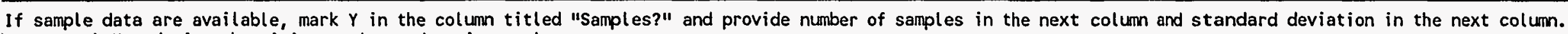
not, mark $N$ and give the minimum value and maximum value.

iditional information or explanations (indicate pertinent contaminant)

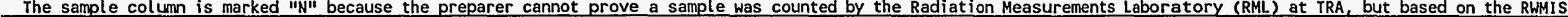

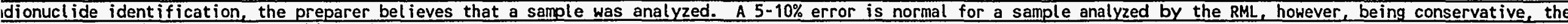
eparer assigned a $20 \%$ error (doubl ing the $10 \%$ error)

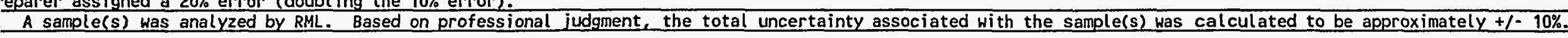


Type of source of information: heck box)

] RWMIS [ ] other database

] sample analysis data

operating records [X] interview

] expert judgment [X] reports

other

Do the estimates of contaminant antities in Part $C$ and $D$ represent: ] best estimate

] worst case

] other

Do the data conflict with RWMIS? (Historical or Present Data only) ] no

] yes

Major unknowns in inventories of ntaminants:

$P$ and MFP were unknown.
2. Details concerning source (names, report no., dates, etc.) L.J. Toomer: ATR-WCR-009-92, ATR-WCR-004-92, ATR-WCR-003-92, ATR-WCR-002-92, ATR-WCR-001-92.

C.R. Amaro, "Amalgamation of Low Level Waste," WROC-WCR-007-93.

4. If other than best estimate, explain why:

\section{If yes, explain why:}

A portion of or all of the measurements were assumed to be taken using a G-M detector which only measures gamma emitters; beta emitters have been added to the RWMIS activity based on scaling factors. Therefore, the total activity will be greater than that in RWMIS.

8. Key assumptions used to deal with the unknowns: MAP and MFP radionuclides were chosen based on EPR1 NP-5077 for dry active waste for PWRs. PWR was chosen because it is similar to ATR, the reactor at TRA. The radionuclides were chosen based on a combination of the following: cross section, half-lives, and their fission product. 
Because of the form (the various types and shapes) of the material, it is not known whether a shipment's activity was determined with a G-M counter or with a NAI detector using smears. Therefore, the true activity and uncertainty is unknown. The G-M counter method has been assumed as the method used to estimate the gamma emitting radionuclides. Although a G-M counter cannot estimate alpha emitting radionuclides, the alpha activities were scaled from the gamma activities. Therefore, the G-M counter correction applies for the alpha emitters, CIDRA calculated best estimate, and upper and lower bounds, accordingly. 
DATA INPUT FOR HISTORICAL DATA TASK FOR RWMC SUBSURFACE DISPOSAL AREA

Preparer: Amaro, C.

Generator: TRA irea or contractor - use code from attached list)

Number of waste stream from this facility: $4 \mathrm{R}$

Type of radioactive waste (check box):

] TRU or suspect TRU

!] LLW

] non-radioactive

Actual years disposed of at SDA: :arting year 1987 Ending year 1993
2. Date prepared:07/03/94

4. Particular facility: 603

(building number - use code from attached list)

6. Waste stream:

Core and loop components.

9. Waste stream volume:

Amount $\quad 22.8360$ Units Cubic meters.

Check box: [ ] annual or [X] total over all years

Check box: [ ] container volume or [X] waste volume

). Comments (specify number of pertinent question):

4. This is the same waste as earlier stream TRA-603-4, but is actually from 670 . 
1. General physical form (see attached list) ther core, reactor vessel, loop component [ ] other (specify)

3. Chemical form:

Metallic (stainless steel and aluminum).

5. Waste container type (see attached list) other.

7. Comments (specify number of pertinent question): 5. Also I, O cannot be defined by the preparer.
Details on physical form (particularly confinement related) Solids, such as loop piping, stainless steel and aluminum pieces, equipment leads, end pile tubes or end boxes from fuel elements.

4. Inner packaging: [ ] plastic bag [X] plastic liner

[ ] metal liner [ ] none [ ] other (specify)

6. Other characteristics" of interest: 


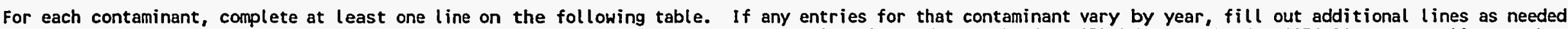

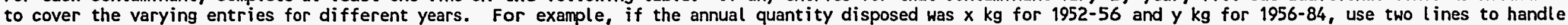
this situation

\begin{tabular}{|c|c|c|c|c|c|c|c|c|c|c|}
\hline Radionuclide & Physical Form & Chemical Form & $\begin{array}{l}\text { (A)nnual/(T)otal } \\
\text { Quantity }\end{array}$ & Unit & $\begin{array}{l}\text { Begin } \\
\text { Year }\end{array}$ & $\begin{array}{l}\text { End } \\
\text { Year }\end{array}$ & $\begin{array}{l}\text { Samp } \\
\text { les? }\end{array}$ & $\begin{array}{c}\text { Minimum } \\
\text { Value/\#Samp }\end{array}$ & $\begin{array}{r}\text { Maximum } \\
\text { Vatue/STD }\end{array}$ & $\begin{array}{l}\text { Basis for } \\
\text { Uncertainty }\end{array}$ \\
\hline$H-3$ & Metallic. & Unknown. & T 440.80000000000 & $\mathrm{Cl}$ & 1987 & 1987 & N & & & See continuation page. \\
\hline C- 14 & Metallic. & Unknown. & T 59.140000000000 & CI & 1987 & 1987 & N & & & See continuation page. \\
\hline $\mathrm{Fe}-55$ & Metallic. & Unknown. & T 10210.000000000 & CI & 1987 & 1987 & N & & & See continuation page. \\
\hline $\mathrm{Ni}-59$ & Metallic. & Unknown. & T 3.0640000000000 & CI & 1987 & 1987 & N & & & See continuation page. \\
\hline $\mathrm{Ni}-63$ & Metallic. & Unknown. & r 1720.0000000000 & CI & 1987 & 1987 & N & & & See continuation page. \\
\hline Co- 60 & Metallic. & Unknown. & T 3602.0000000000 & CI & 1987 & 1987 & N & & & See continuation page. \\
\hline $\mathrm{Sr}-90$ & Metallic. & Unknown. & T 4.9460000000000 & $\mathrm{CI}$ & 1987 & 1987 & N & & & See continuation page. \\
\hline TC-99 & Metallic. & Unknown. & T. .96770000000000 & CI & 1987 & 1987 & N & & & See continuation page. \\
\hline$I-129$ & Metallic. & Unknown. & T. .00025164000000 & CI & 1987 & 1987 & N & & & See continuation page. \\
\hline
\end{tabular}

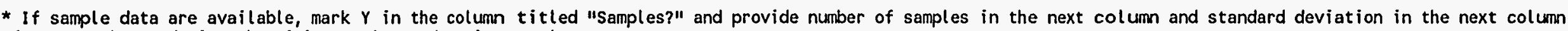
If not, mark $N$ and give the minimum value and maximum value,

Additional information or explanations (indicate pertinent contaminant)

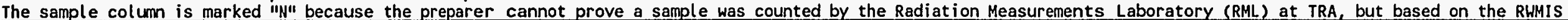

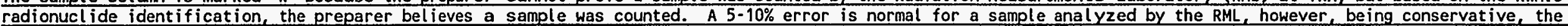
preparer assigned a $20 \%$ error (doubl ing the $10 \%$ error). 
or each contaminant, complete at least one line on the following table. If any entries for that contaminant vary by year, fill out additional lines as needed

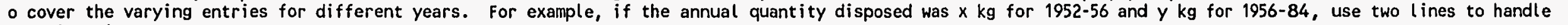
his situation.

\begin{tabular}{|c|c|c|c|c|c|c|c|c|c|c|}
\hline Radionucl ide & Physical Form & Chemical Form & $\begin{array}{l}\text { (A)nnual/(T)otal } \\
\text { Quantity }\end{array}$ & Unit & $\begin{array}{l}\text { Begin } \\
\text { Year }\end{array}$ & $\begin{array}{l}\text { End } \\
\text { Year }\end{array}$ & $\begin{array}{l}\text { Samp } \\
\text { les? }\end{array}$ & $\begin{array}{c}\text { Minimum } \\
\text { Value/\#Samp }\end{array}$ & $\begin{array}{r}\text { Maximum } \\
\text { Value/STD }\end{array}$ & $\begin{array}{l}\text { Basis for } \\
\text { Uncertainty }\end{array}$ \\
\hline Cs -137 & Metallic. & Unknown. & T 1075.0000000000 & $\mathrm{CI}$ & 1987 & 1987 & N & & & See cont inuation page. \\
\hline $\mathrm{Ce}-144$ & Metallic. & Unknown. & T 25.270000000000 & CI & 1987 & 1987 & N & & & See continuation page. \\
\hline Eu- 954 & Metallic. & Unknown. & T.01559000000000 & $\mathrm{CI}$ & 1987 & 1987 & N & & & See continuation page. \\
\hline Eu- 155 & Metallic. & Unknown. & T 50.530000000000 & CI & 1987 & 1987 & N & & & See continuation page. \\
\hline$U-234$ & Metallic. & Unknown. & T.01129000000000 & $\mathrm{Cl}$ & 1987 & 1987 & $\mathbf{N}$ & & & See continuation page. \\
\hline$U-235$ & Metallic. & Unknown. & T.00024190000000 & CI & 1987 & 1987 & $N$ & & & See continuation page. \\
\hline $\mathrm{U}-236$ & Metallic. & Unknown. & T. .00430100000000 & CI & 1987 & 1987 & N & & & See continuation page. \\
\hline Np-237 & Metallic. & Unknown. & T. .00698900000000 & $\mathrm{Cl}$ & 1987 & 1987 & $\mathbf{N}$ & & & See continuation page. \\
\hline Pu-238 & Metallic. & Unknown. & T. .29030000000000 & CI & 1987 & 1987 & N & & & See continuation page. \\
\hline
\end{tabular}

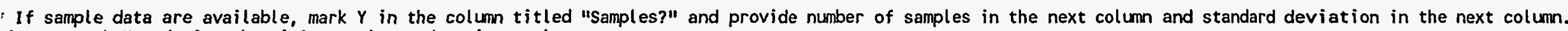
$f$ not, mark $N$ and give the minimum value and maximum value.

idditional information or explanations (indicate pertinent contaminant)

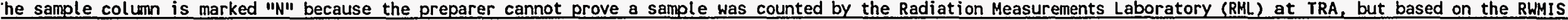

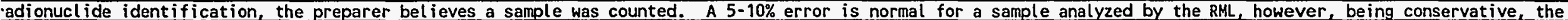
reparer assigned a $20 \%$ error (doubling the $10 \%$ error). 


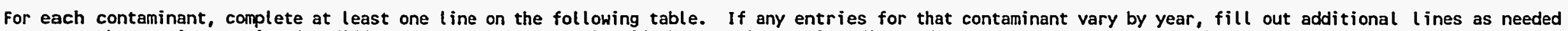

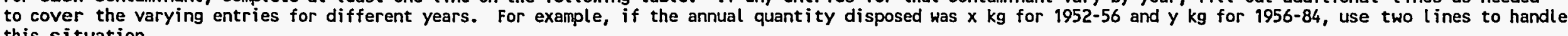
this situation.

\begin{tabular}{|c|c|c|c|c|c|c|c|c|c|c|}
\hline Radionuclide & Physical Form & Chemical form & $\begin{array}{l}\text { (A)nnual / ( T )otal } \\
\text { Quant ity }\end{array}$ & Unit & $\begin{array}{l}\text { Begin } \\
\text { Year }\end{array}$ & $\begin{array}{l}\text { End } \\
\text { Year }\end{array}$ & $\begin{array}{l}\text { Samp } \\
\text { les? }\end{array}$ & $\begin{array}{c}\text { Minimum } \\
\text { Value/\#Samp }\end{array}$ & $\begin{array}{c}\text { Maximum } \\
\text { Value/STD }\end{array}$ & $\begin{array}{l}\text { Basis for } \\
\text { Uncertainty }\end{array}$ \\
\hline Te-125m & Metallic. & Unknown. & T 1.0000000000000 & CI & 1991 & 1991 & N & $-20 \%$ & $+20 \%$ & See comment below. \\
\hline $2 n-65$ & Metallic. & Unknown. & T 801.55000000000 & $\mathrm{CI}$ & 1991 & 1991 & N & $-20 \%$ & $+20 \%$ & See comment below. \\
\hline$C-14$ & Metallic. & Unknown. & $T .00037700000000$ & CI & 1992 & 1992 & N & $-20 \%$ & $+20 \%$ & See comment below. \\
\hline Co-60 & Metallic. & Unknown. & T 12.570000000000 & CI & 1992 & 1992 & N & $-20 \%$ & $+20 \%$ & See comment below. \\
\hline $\mathrm{Cr}-51$ & Metallic. & Unknown. & T. .07000000000000 & CI & 1992 & 1992 & N & $-20 \%$ & $+20 \%$ & See comment below. \\
\hline $\mathrm{Fe}-55$ & Metallic. & Unknown. & T 64.900000000000 & CI & 1992 & 1992 & N & $-20 \%$ & $+20 \%$ & See comment below. \\
\hline$H-3$ & Metallic. & Unknown. & T. .00000000060000 & CI & 1992 & 1992 & N & $-20 \%$ & $+20 \%$ & See comment below. \\
\hline Mn-54 & Metallic. & Unknown. & T 8.9800000000000 & $\mathrm{CI}$ & 1992 & 1992 & N & $-20 \%$ & $+20 \%$ & See comment below. \\
\hline $\mathrm{Nb}-94$ & Metallic. & Unknown. & $T .00000002540000$ & CI & 1992 & 1992 & N & $-20 \%$ & $+20 \%$ & See comment below. \\
\hline
\end{tabular}

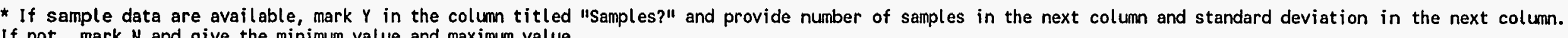
If not, mark $N$ and give the minimum value and maximum value.

Additional information or explanations (indicate pertinent contaminant)

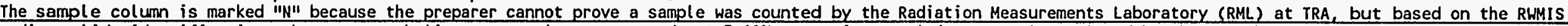

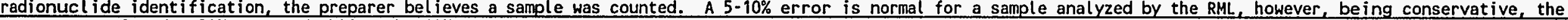
preparer assigned a $20 \%$ error (doubling the 10\% error). 
1. Type of source of information: (check box)

\section{[X] RWMIS [ ] other database}

[ ] sample analysis data

[ ] operating records [ ] interview

[ ] expert judgment [ ] reports

] other

3. Do the estimates of contaminant quantities in Part $C$ and $D$ represent: [X] best estimate

[] worst case

] other

5. Do the data conflict with RWMIS? (Historical or Present Data only) [ ] no

[X] yes

7. Major unknowns in inventories of contaminants:

MAP and MFP were unknown.
2. Details concerning source (names, report no., dates, etc.)

4. If other than best estimate, explain why:

6. If yes, explain why:

A portion of or all of the measurements were assumed to be taken using a G-M detector which only measures gamma emitters; beta emitters have been added to the RWMIS activity based on scaling factors. Therefore, the activity will be greater than that in RWMIS.

8. Key assumptions used to deal with the unknowns: MAP and MFP radionuclides were chosen based on EPR1 NP-5077 for dry active waste for PWRs. PWR was chosen because it is similar to ATR, the reactor at TRA. The radionuclides were chosen based on a combination of the following: cross section, half-lives, and their fission products. 
ontinuation of Part $\underline{D}$

Column or Question Number or Title Uncertainty basis.

cause of the form (the various types and shapes) of the material, it is not known whether a ipment's activity was determined with a G-M counter or with a NAI detector using smears. ierefore, the true activity and uncertainty is unknown. The G-M counter method has been assumed as le method used to estimate the gamma emitting radionuclides. Although a G-M counter cannot timate alpha emitting radionuclides, the alpha activities were scaled from the gamma activities. erefore, the G-M counter correction applies for the alpha emitters, CIDRA calculated best timate, and upper and lower bounds, accordingly. 
DATA INPUT FOR HISTORICAL DATA TASK FOR RWMC SUBSURFACE DISPOSAL AREA

'ART A - GENERAL INFORMATION RDT - 271

- Preparer: Amaro, C.

i. Generator: TRA

area or contractor - use code from attached list)

i. Number of waste stream from this facility: $6 \mathrm{R}$

' Type of radioactive waste (check box):

] TRU or suspect TRU

X] LLW

non-radioactive

i. Actual years disposed of at SDA:

itarting year 1985 Ending year 1991
2. Date prepared: $07 / 03 / 94$

4. Particular facility: 603

(building number - use code from attached list)

6. Waste stream:

sludge.

.0. Comments (specify number of pertinent question):

4. Waste actually came from building 732 , but is identical to waste generated previously as stream TRA-603-6. Also, the TRA-ERP facility is unknown, as the preparer interviewed various people in an effort to locate this facility.

9. Waste stream volume:

Amount 7.6650 Units Cubic meters.

Check box: [ ] annual or [X] total over all years

Check box: [ ] container volume or [X] waste volume 
General physical form (see attached list) 2. Details on physical form(particularly confinement related) udge.

] other (specify)

(2)

\section{Chemical form:}

known.

Waste container type (see attached list) oden box.

Comments (specify number of pertinent question):

5. Also RDL.
4. Inner packaging: [ ] plastic bag [ ] plastic Iiner [ ] metal liner [] none [X] other (specify) Unknown.

6. Other characteristics of interest: 
- each contaminant, complete at least one line on the following table. If any entries for that contaminant vary by year, fill out additional lines as needed

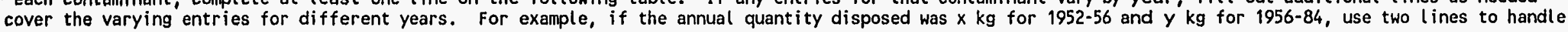
is situation.

\begin{tabular}{|c|c|c|c|c|c|c|c|c|c|c|}
\hline Radionuclide & Physical form & Chemical Form & $\begin{array}{l}\text { (A)nnual/(T) otal } \\
\text { Quant ity }\end{array}$ & Unit & $\begin{array}{l}\text { Begin } \\
\text { Year }\end{array}$ & $\begin{array}{l}\text { End } \\
\text { Year }\end{array}$ & $\begin{array}{l}\text { Samp } \\
\text { l'es? }\end{array}$ & $\begin{array}{c}\text { Minimum } \\
\text { value/\#Samp }\end{array}$ & $\begin{array}{r}\text { Maximum } \\
\text { value/STD }\end{array}$ & $\begin{array}{l}\text { Basis for } \\
\text { Uncertainty }\end{array}$ \\
\hline $\mathrm{H}-3$ & Unknown. & Unknown. & $T .00001000000000$ & Ci & 1986 & 1986 & N & & & See cont inuation page. \\
\hline$C-14$ & Unknown. & Unknown. & T. .00000100000000 & CI & 1986 & 1986 & $\mathbf{N}$ & & & See continuation page. \\
\hline $\mathrm{Fe}-55$ & Unknown. & Unknown. & $T .00007700000000$ & CI & 1986 & 1986 & $\mathbf{N}$ & & & See continuation page. \\
\hline $\mathrm{Ni}-59$ & Unknown. & Unknown. & T. .00000040000000 & CI & 1986 & 1986 & N & & & See cont inuation page. \\
\hline $\mathrm{Ni}-63$ & Unknown. & Unknown. & $T .00003200000000$ & CI & 1986 & 1986 & N & & & See continuation page. \\
\hline Co- 60 & Unknown. & Unknown. & $T .00008700000000$ & CI & 1986 & 1986 & N & & & See continuation page. \\
\hline $\mathrm{Sr}-90$ & Unknown. & Unknown. & $\mathrm{T} .00000006400000$ & $\mathrm{CI}$ & 1986 & 1986 & N & & & See continuation page. \\
\hline Tc-99 & Unknown. & Unknown. & $T .00000001000000$ & CI & 1986 & 1986 & $\mathbf{N}$ & & & See continuation page. \\
\hline$\downarrow-129$ & Unknown. & Unknown. & $\mathrm{T}$ & CI & 1986 & 1986 & $\mathbf{N}$ & & & See cont inuation page. \\
\hline
\end{tabular}

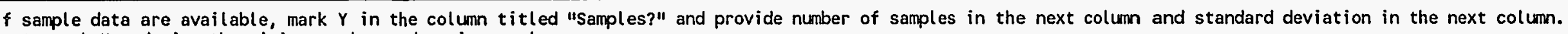
not, mark $N$ and give the minimum value and maximum value.

litional information or explanations (indicate pertinent contaminant)

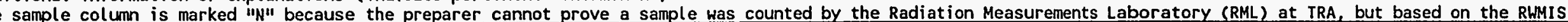

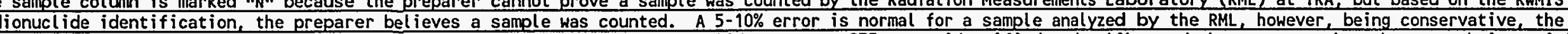

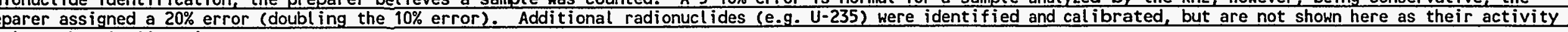
less than $1 E-11$ curies. 
- Type of source of information: check box)

X] RWMIS [ ] other database

] sample analysis data

] operating records [ ] interview

] expert judgment [ ] reports

] other

- Do the estimates of contaminant

ruantities in Part $C$ and $D$ represent:

$\mathrm{X}]$ best estimate

] worst case

] other

- Do the data conflict with RWMIS? (Historical or Present Data only) ] no

$x]$ yes

- Major unknowns in inventories of ontaminants:

IAP arid MFP were unknown.
2. Details concerning source (names, report no., dates, etc.)

4. If other than best estimate, explain why:

6. If yes, explain why:

A portion of or all of the measurements were assumed to be taken using a G-M detector which only measures gamma emitters; beta emitters have been added to the RWMIS activity based on scaling factors. Therefore, the total activity will be greater than that in RWMIS.

8. Key assumptions used to deal with the unknowns: MAP and MFP radionuclides were chosen based on EPR1 NP-5077 for dry active waste for PWRs. PWR was chosen because it is similar to ATR, the reactor at TRA. The radionuclides were chosen based on a combination of the following: cross section, half-lives, and their fission products. 
3ecause of the form (the various types and shapes) of the material, it is not known whether a hipment's activity was determined with a G-M counter or with a NAI detector using smears. Cherefore, the true activity and uncertainty is unknown. The G-M counter method has been assumed as -he method used to estimate the gamma emitting radionuclides. Although a G-M counter cannot stimate alpha emitting radionuclides, the alpha activities were scaled from the gamma activities. cherefore, the G-M counter correction applies for the alpha emitters, CIDRA calculated best stimate, and upper and lower bounds, accordingly. 


\section{RT A - GENERAL INFORMATION RDT - 272}

Preparer: Amaro, $\mathrm{C}$.

Generator: TRA

rea or contractor - use code from attached list)

Number of waste stream from this facility: $7 \mathrm{R}$

Type of radioactive waste (check box):

] TRU or suspect TRU

$L L W$

non-radioactive

Actual years disposed of at SDA: arting year 1987 Ending year 1987

Comments (specify number of pertinent question): 4. Waste actually came from Building 632 , but is identical to the waste previously generated in stream TRA-603-7.
2. Date prepared: $07 / 03 / 94$

4. Particular facility: 603

(building number - use code from attached list)

6. Waste stream:

Glass.

9. Waste stream volume: Amount 0.0284 Units Cubic meters.

Check box: [ ] annual or [X] total over all years

Check box: [] container volume or [X] waste volume 
1. General physical form (see attached list) Jlass.

[ ] other (specify)

3. Chemical form:

Sio2.

5. Waste container type (see attached list) Insert.

7. Comments (specify number of pertinent question):
2. Details on physical form(particularly confinement related) This stream is made of glass.

4. Inner packaging: [ ] plastic bag [X] plastic liner [ ] metal liner [ ] none [ ] other (specify)

6. Other characteristics of interest: 


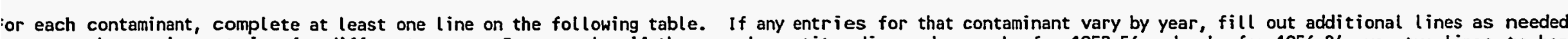

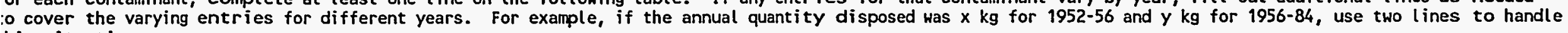
his situation.

\begin{tabular}{|c|c|c|c|c|c|c|c|c|c|c|}
\hline Radionucl ide & Physical Form & Chemical form & $\begin{array}{l}\text { (A)nnual } /(T) \text { otal } \\
\text { Quant ity }\end{array}$ & Unit & $\begin{array}{l}\text { Begin } \\
\text { Year }\end{array}$ & $\begin{array}{l}\text { End } \\
\text { Year }\end{array}$ & $\begin{array}{l}\text { Samp } \\
\text { les? }\end{array}$ & $\begin{array}{c}\text { Minimum } \\
\text { Value/\#Samp }\end{array}$ & $\begin{array}{r}\text { Maximum } \\
\text { Value/STD }\end{array}$ & $\begin{array}{l}\text { Basis for } \\
\text { Uncertainty }\end{array}$ \\
\hline $\mathrm{H}-3$ & Glass. & sio2. & T.68000000000000 & CI & 1987 & 1987 & N & & & See continuation page. \\
\hline C- 14 & Glass. & sio2. & T. .09122000000000 & CI & 1987 & 1987 & N & & & See continuation page. \\
\hline $\mathrm{Fe}-55$ & Glass. & sio2. & T 15.760000000000 & CI & 1987 & 1987 & N & & & See continuation page. \\
\hline $\mathrm{Ni}-59$ & Glass. & sio2. & T. .00472700000000 & CI & 1987 & 1987 & N & & & See continuation page. \\
\hline $\mathrm{Ni}-63$ & Glass. & sio2. & T 2.654000000000 & CI & 1987 & 1987 & N & & & See continuation page. \\
\hline Co- 60 & Glass. & sio2. & T 5.556000000000 & CI & 1987 & 1987 & N & & & See continuation page. \\
\hline Sr-90 & Glass. & sio2. & $T .00763000000000$ & $\mathrm{CI}$ & 1987 & 1987 & N & & & See cont inuation page. \\
\hline TC- 99 & Glass. & sio2. & T. .00149300000000 & CI & 1987 & 1987 & N & & & See continuation page. \\
\hline I -129 & Glass. & sio2. & T.00000038808000 & CI & 1987 & 1987 & N & & & See continuation page. \\
\hline
\end{tabular}

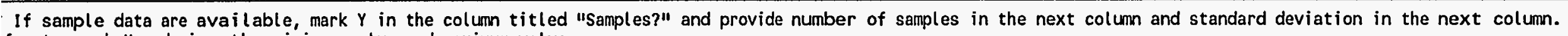
$f$ not, mark $N$ and give the minimum value and maximum value.

dditional information or explanations (indicate pertinent contaminant)

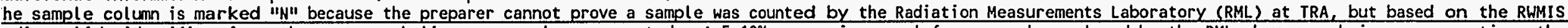

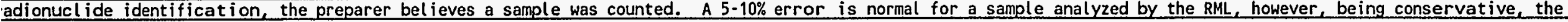
reparer assigned a $20 \%$ error (doubling the $10 \%$ error). 
each contaminant, complete at least one line on the following table. If any entries for that contaminant vary by year, fill out additional lines as needed

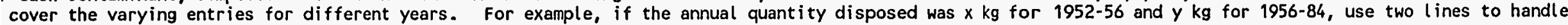
is situation.

\begin{tabular}{|c|c|c|c|c|c|c|c|c|c|c|}
\hline Radionuctide & Physical Form & Chemical form & $\begin{array}{l}\text { (A)nnual/( } T \text { ) otal } \\
\text { Quant ity }\end{array}$ & Unit & $\begin{array}{l}\text { Begin } \\
\text { Year }\end{array}$ & $\begin{array}{l}\text { End } \\
\text { Year }\end{array}$ & $\begin{array}{l}\text { Samp } \\
\text { les? }\end{array}$ & $\begin{array}{c}\text { Minimum } \\
\text { Value/\#Samp }\end{array}$ & $\begin{array}{r}\text { Maximum } \\
\text { Value/STD }\end{array}$ & $\begin{array}{l}\text { Basis for } \\
\text { Uncertainty }\end{array}$ \\
\hline Cs-137 & Glass. & sior. & T 1.6590000000000 & CI & 1987 & 1987 & N & & & See continuation page. \\
\hline $\mathrm{Ce}-144$ & Glass. & sio2. & $T .03898000000000$ & $\mathrm{Cl}$ & 1987 & 1987 & N & & & See cont inuation page. \\
\hline Eu- 154 & Glass. & sio2. & T. .00002405000000 & CI & 1987 & 1987 & N & & & See cont inuation page. \\
\hline Eu-155 & Glass. & sioz. & T. .07795000000000 & CI & 1987 & 1987 & N & & & See continuation page. \\
\hline U-234 & Glass. & sio2. & T. .00001742000000 & CI & 1987 & 1987 & N & & & See continuation page. \\
\hline$U-235$ & Glass. & sio2. & $T .00000037320000$ & CI & 1987 & 1987 & N & & & See cont inuation page. \\
\hline$U-236$ & Glass. & sio2. & $T .00000663400000$ & CI & 1987 & 1987 & $N$ & & & See continuation page. \\
\hline Np-237 & Glass. & sio2. & T. .00001078000000 & Cl & 1987 & 1987 & N & & & See cont inuation page. \\
\hline Pu-238 & Glass. & sio2. & $T .00044780000000$ & $\mathrm{CI}$ & 1987 & 1987 & N & & & See continuation page. \\
\hline
\end{tabular}

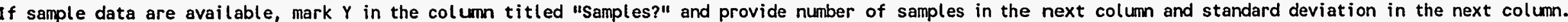
not, mark $N$ and give the minimum value and maximum value.

ditional information or explanations (indicate pertinent contaminant)

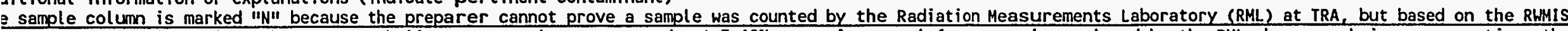

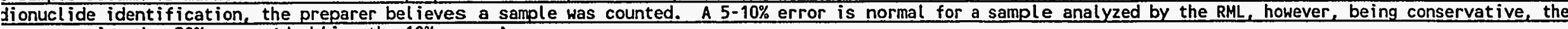
eparer assigned a $20 \%$ error (doubling the 10\% error). 


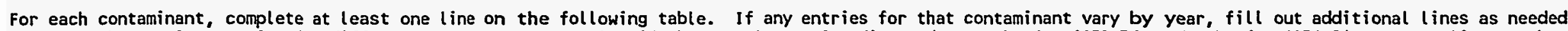

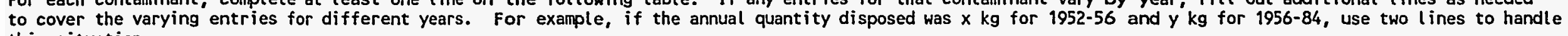
this situation.

\begin{tabular}{|c|c|c|c|c|c|c|c|c|c|c|}
\hline Radionuclide & Physical Form & Chemical Form. & $\begin{array}{l}\text { (A)nnual/(T)otal } \\
\text { Quantity }\end{array}$ & Unit & $\begin{array}{l}\text { Begin } \\
\text { Year }\end{array}$ & $\begin{array}{l}\text { End } \\
\text { Year }\end{array}$ & $\begin{array}{l}\text { Samp } \\
\text { Les? }\end{array}$ & $\begin{array}{c}\text { Minimum } \\
\text { Value/\#Samp }\end{array}$ & $\begin{array}{r}\text { Maximum } \\
\text { Value/STD }\end{array}$ & $\begin{array}{l}\text { Basis for } \\
\text { Uncertainty }\end{array}$ \\
\hline Pu-239 & Glass. & sio2. & T. .00044780000000 & CI & 1987 & 1987 & $\mathbf{N}$ & & & See continuation page. \\
\hline $\mathrm{Pu}-240$ & Glass. & sion. & T. .00004727000000 & CI & 1987 & 1987 & $\mathbf{N}$ & & & See continuation page. \\
\hline Pu-241 & Glass. & sio2. & T. .04893000000000 & CI & 1987 & 1987 & $\mathbf{N}$ & & & See continuation page. \\
\hline$A m-241$ & Glass. & sio2. & $T .00022390000000$ & CI & 1987 & 1987 & $\mathbf{N}$ & & & See continuation page. \\
\hline $\mathrm{Cm}-242$ & Glass. & sio2. & T. .00022390000000 & $\mathrm{CI}$ & 1987 & 1987 & N & & & See continuation page. \\
\hline $\mathrm{Cm}-244$ & Glass. & sio2. & T. .00020730000000 & CI & 1987 & 1987 & N & & & See continuation page. \\
\hline Cs -134 & Glass. & sio2. & $T .59000000000000$ & CI & 1987 & 1987 & $\mathbf{N}$ & $-20 \%$ & $+20 \%$ & See comment below. \\
\hline Gd-153 & Glass. & sion. & T. .98000000000000 & $\mathrm{Cl}$ & 1987 & 1987 & $\mathbf{N}$ & $-20 \%$ & $+20 \%$ & See comment below. \\
\hline I $r-192$ & Glass. & sio2. & $T .49000000000000$ & $\mathrm{CI}$ & 1987 & 1987 & $\mathbf{N}$ & $-20 \%$ & $+20 \%$ & See comment below. \\
\hline
\end{tabular}

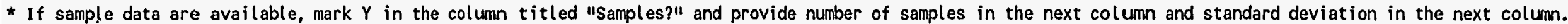
If not, mark $N$ and give the minimum value and maximum value.

Additional information or explanations (indicate pertinent contaminant)

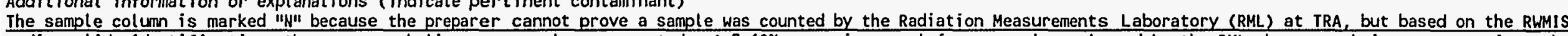

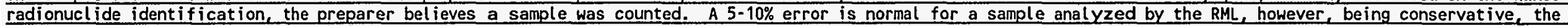
preparer assigned a $20 \%$ error (doubling the $10 \%$ error) 
. Type of source of information: (check box)

X] RWMIS [ ] other database

] sample analysis data

] operating records [ ] interview

] expert judgment [ ] reports

] other

- Do the estimates of contaminant

duantities in Part $C$ and $D$ represent:

$x]$ best estimate

] worst case

] other

- Do the data conflict with RWMIS?

(Historical or Present Data only)

] no

$\mathrm{x}]$ yes

- Major unknowns in inventories of :ontaminants:

IAP and MFP were unknown.
2. Details concerning source (names, report no., dates, etc.)

4. If other than best estimate, explain why:

6. If yes, explain why:

A portion of or all of the measurements were assumed to be taken using a G-M detector which only measures gamma emitters; beta emitters have been added to the RWMIS activity based on scaling factors. Therefore, the total activity will be greater than that in RWMIS.

8. Key assumptions used to deal with the unknowns: MAP and MFP radionuclides were chosen based on EPR1 NP-5077 for dry active waste for PWRs. PWR was chosen because it is similar to ATR, the reactor at TRA. The radionuclides were chosen based on a combination of the following: cross section, half-lives, and their fission products. 
3ecause of the form (the various types and shapes) of the material, it is not known whether a hipment's activity was determined with a G-M counter or a NAI detector using smears. Therefore, he true activity and uncertainty is unknown. The G-M counter method has been assumed as the method ised to estimate the gamma emitting radionuclides. Although a G-M counter cannot estimate alpha mitting radionuclides, the alpha activities were scaled from the gamma activities. Therefore, the i-M counter corrections applies for the alpha emitters, CIDRA calculated best estimate, and upper ind lower bounds, accordingly. 
2. Date prepared: $07 / 03 / 94$

4. Particular facility: 603

(building number - use code from attached list)

6. Waste stream:

Metal, such as aluminum and stainless steel.

9. Waste stream volume:

Amount 273.0580 Units Cubic meters.

Check box: [ ] annual or [X] total over all years

Check box: [ ] container volume or [X] waste volume

. Comments (specify number of pertinent question): 4. Also $632,604,635,670,661,605,637,732$, and TRANSA. The facility, TRANSA, could not be identified by the preparer. 


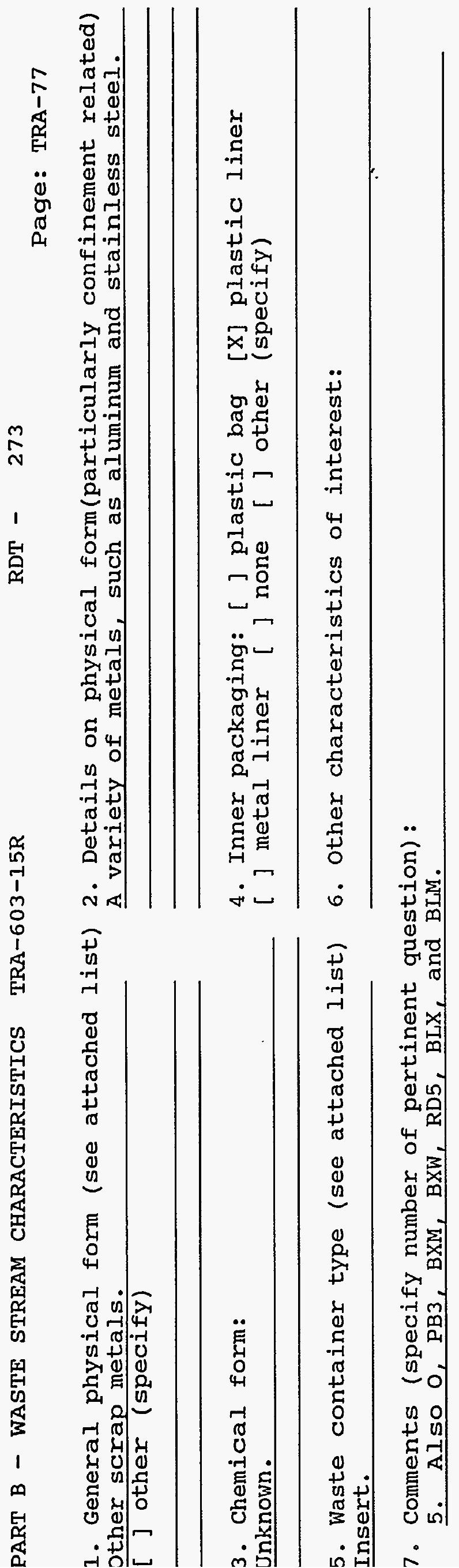


$r$ each contaminant, complete at least one line on the following table. If any entries for that contaminant vary by year, fill out additional lines as needed

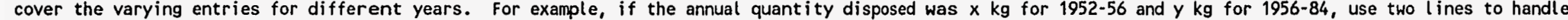
is situation.

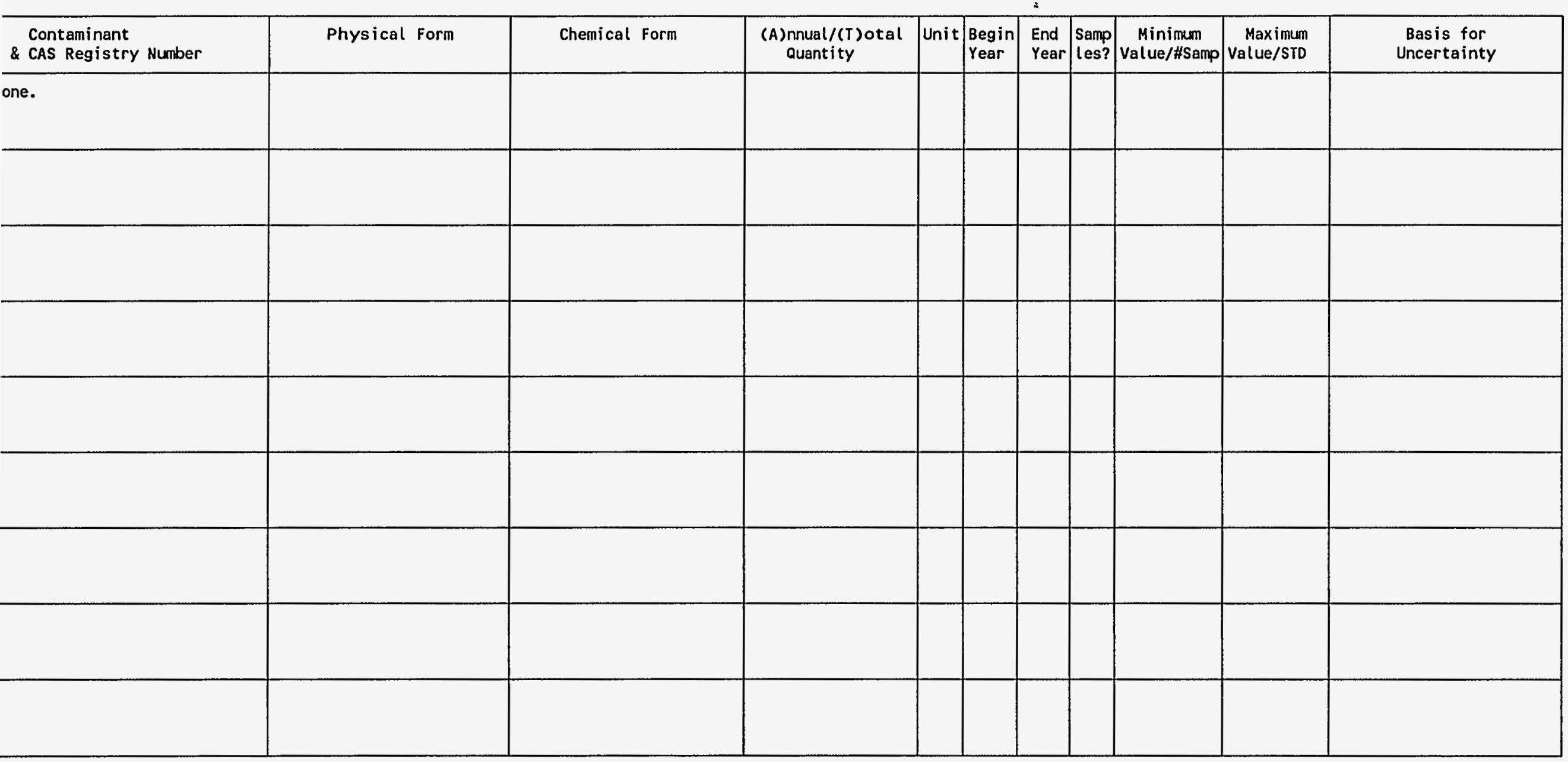

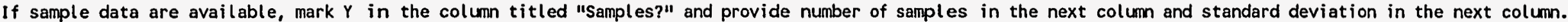
not, mark $N$ and give the minimum value and maximum value.

ditional information or explanations (indicate pertinent contaminant) 
each contaminant, complete at least one line on the following table. If any entries for that contaminant vary by year, fill out additional lines as needed

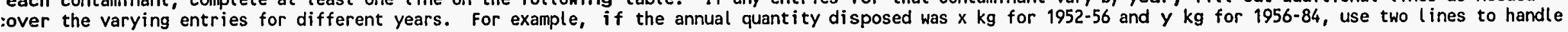
isituation.

\begin{tabular}{|c|c|c|c|c|c|c|c|c|c|c|}
\hline Radionuclide & Physical Form & Chemical Form & $\begin{array}{l}\text { (A)nnual / (T) otal } \\
\text { Quant ity }\end{array}$ & Unit & $\begin{array}{l}\text { Begin } \\
\text { Year }\end{array}$ & $\begin{array}{l}\text { End } \\
\text { Year }\end{array}$ & $\begin{array}{l}\text { Samp } \\
\text { les? }\end{array}$ & $\begin{array}{c}\text { Minimum } \\
\text { value/\#Samp }\end{array}$ & $\begin{array}{r}\text { Maximum } \\
\text { Value/STD }\end{array}$ & $\begin{array}{l}\text { Basis for } \\
\text { Uncertainty }\end{array}$ \\
\hline Tc-99 & Metallic. & Unknown. & $T .00000003600000$ & $\mathrm{Cl}$ & 1985 & 1985 & N & & & See continuation page. \\
\hline $1-129$ & Metallic. & Unknown. & T. .00000010400000 & $\mathrm{Cl}$ & 1985 & 1985 & N & & & See continuation page. \\
\hline Cs -137 & Metallic. & Unknown. & $T .00004000000000$ & $\mathrm{CI}$ & 1985 & 1985 & N & & & See cont inuation page. \\
\hline $\mathrm{Ce}-144$ & Metallic. & Unknown. & $T .00000094000000$ & $\mathrm{Cl}$ & 1985 & 1985 & N & & & See cont inuation page. \\
\hline Eu-154 & Metallic. & Unknown. & $T .00000000060000$ & $\mathrm{CI}$ & 1985 & 1985 & N & & & See cont inuation page. \\
\hline Eu-155 & Metallic. & Unknown. & $T .00000188000000$ & CI & 1985 & 1985 & N & & & See cont inuation page. \\
\hline$U-234$ & Metallic. & Unknown. & $T .00000000040000$ & CI & 1985 & 1985 & N & & & See cont inuation page. \\
\hline$U-236$ & Metallic. & Unknown. & $T .00000000020000$ & CI & 1985 & 1985 & N & & & See cont inuation page. \\
\hline$N p-237$ & Metallic. & Unknown. & T. .00000000030000 & $\mathrm{CI}$ & 1985 & 1985 & $\mathbf{N}$ & & & See continuation page. \\
\hline
\end{tabular}

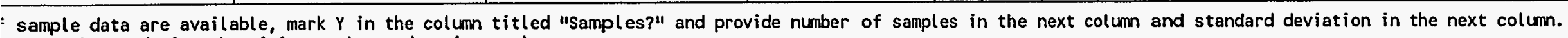
10t, mark $N$ and give the minimum value and maximum value.

tional information or explanations (indicate pertinent contaminant)

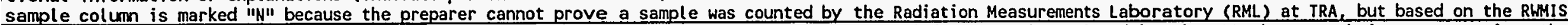

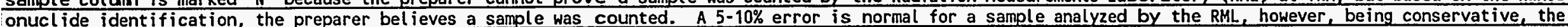

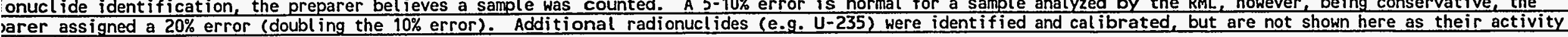
less than $1 \mathrm{E}-11$ curies. 
- each contaminant, complete at least one line on the following table. If any entries for that contaminant vary by year, fill out additional lines as needed

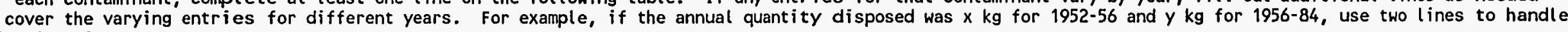
is situation.

\begin{tabular}{|c|c|c|c|c|c|c|c|c|c|c|}
\hline Radionuclide & Physical Form & Chemical form & $\begin{array}{l}\text { (A)nnual /(T)otal } \\
\text { Quant ity }\end{array}$ & Unit & $\begin{array}{l}\text { Begin } \\
\text { Year }\end{array}$ & $\begin{array}{l}\text { End } \\
\text { Year }\end{array}$ & $\begin{array}{l}\text { Samp } \\
\text { les? }\end{array}$ & $\begin{array}{c}\text { Minimum } \\
\text { Value/\#Samp }\end{array}$ & $\begin{array}{r}\text { Maximum } \\
\text { Value/STD }\end{array}$ & $\begin{array}{l}\text { Basis for } \\
\text { Uncertainty }\end{array}$ \\
\hline Cs-134 & Metallic. & Unknown. & T. .00370000000000 & $\mathrm{Cl}$ & 1985 & 1985 & N & $-20 \%$ & $+20 \%$ & See comment below. \\
\hline Cs -137 & Metallic. & Unknown. & T.13000000000000 & $\mathrm{CI}$ & 1985 & 1985 & N & $-20 \%$ & $+20 \%$ & See comment below. \\
\hline$M n-54$ & Metallic. & Unknown. & T.00220000000000 & $\mathrm{Cl}$ & 1985 & 1985 & N & $-20 \%$ & $+20 \%$ & See comment below. \\
\hline$H-3$ & Metallic. & Unknown. & T.33520000000000 & $\mathrm{CI}$ & 1987 & 1987 & N & & & See continuation page. \\
\hline$c-14$ & Metallic. & Unknown. & $T .04497000000000$ & $\mathrm{Cl}$ & 1987 & 1987 & N & & & See continuation page. \\
\hline $\mathrm{Fe}-55$ & Metallic. & Unknown. & T 7.7670000000000 & CI & 1987 & 1987 & N & & & See continuation page. \\
\hline $\mathrm{Ni}-59$ & Metallic. & Unknown. & T.00233000000000 & CI & 1987 & 1987 & N & & & See continuation page. \\
\hline $\mathrm{Ni}-63$ & Metallic. & Unknown. & T 1.3080000000000 & $\mathrm{CI}$ & 1987 & 1987 & N & & & See continuation page. \\
\hline Co-60 & Metallic. & Unknown. & T 2.7390000000000 & CI & 1987 & 1987 & N & & & See continuation page. \\
\hline
\end{tabular}

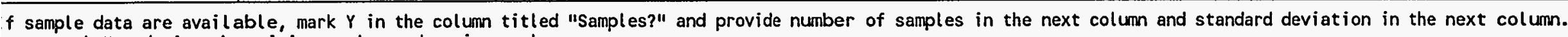
not, mark $N$ and give the minimum value and maximum value.

ditional information or explanations (indicate pertinent contaminant)

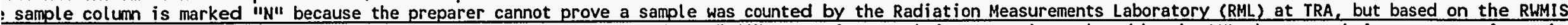

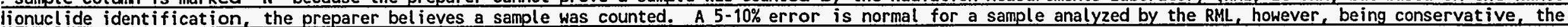

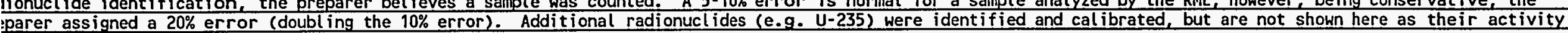
Less than 1E-11 curies. 
or each contaminant, complete at least one line on the following table. If any entries for that contaminant vary by year, fill out additional lines as needed cover the varying entries for different years. For example, if the annual quantity disposed was $x \mathrm{~kg}$ for $1952-56$ and y $\mathrm{kg}$ for 1956-84, use two lines to handle nis situation.

\begin{tabular}{|c|c|c|c|c|c|c|c|c|c|c|}
\hline Radionucl ide & Physical Form & Chemical form & $\begin{array}{l}\text { (A)nnual/(T)otal } \\
\text { Quantity }\end{array}$ & Unit & $\begin{array}{l}\text { Begin } \\
\text { Year }\end{array}$ & $\begin{array}{l}\text { End } \\
\text { Year }\end{array}$ & $\begin{array}{l}\text { Samp } \\
\text { les? }\end{array}$ & $\begin{array}{c}\text { Minimum } \\
\text { Value/\#Samp }\end{array}$ & $\begin{array}{r}\text { Maximum } \\
\text { Value/STD }\end{array}$ & $\begin{array}{l}\text { Basis for } \\
\text { Uncertainty }\end{array}$ \\
\hline $\mathrm{Cm}-242$ & Metallic. & Unknown. & T. .00006695000000 & CI & 1988 & 1988 & N & & & See continuation page. \\
\hline $\mathrm{Cm}-244$ & Metallic. & Unknown. & T. .00006199000000 & $\mathrm{CI}$ & 1988 & 1988 & N & & & See continuation page. \\
\hline $\mathrm{Cr}-51$ & Metallic. & Unknown. & T 13.993000000000 & CI & 1988 & 1988 & N & $-20 \%$ & $+20 \%$ & See comment below. \\
\hline Co- 60 & Metallic. & Unknown. & Т . .39233000000000 & CI & 1991 & 1991 & N & $-20 \%$ & $+20 \%$ & See comment below. \\
\hline Cs-137 & Metallic. & Unknown. & T 51.665000000000 & CI & 1991 & 1991 & N & $-20 \%$ & $+20 \%$ & See comment below. \\
\hline Am-241 & Metallic. & Unknown. & T 3.1009137067000 & $\mathrm{CI}$ & 1991 & 1991 & N & $-20 \%$ & $+20 \%$ & See comment below. \\
\hline Eu-152 & Metallic. & Unknown. & Т. .00999191060000 & $\mathrm{CI}$ & 1991 & 1991 & N & $-20 \%$ & $+20 \%$ & See comment below. \\
\hline Eu-154 & Metallic. & Unknown. & Т. .00070470000000 & CI & 1991 & 1991 & N & $-20 \%$ & $+20 \%$ & See comment below. \\
\hline Eu-155 & Metallic. & Unknown. & Т. .33891600000000 & $\mathrm{CI}$ & 1991 & 1991 & N & $-20 \%$ & $+20 \%$ & See comment below. \\
\hline
\end{tabular}

If sample data are available, mark $Y$ in the column titled "Samples?" and provide number of samples in the next column and standard deviation in the next column. not, mark $N$ and give the minimum value and maximum value.

editional information or explanations (indicate pertinent contaminant)

e sample column is marked "N" because the preparer cannot prove a sample was counted by the Radiation Measurements Laboratory (RML) at TRA, but based on the RLMIS adionuclide identification, the preparer believes a sample was counted. A 5-10\% error is normal for a sample analyzed by the RML, however, being conservative, the eparer assigned a $20 \%$ error (doubling the 10\% error). Additional radionuclides (e.g. U-235) were identified and calibrated, but are not shown here as their activity as less than $1 \mathrm{E}-11$ curies 


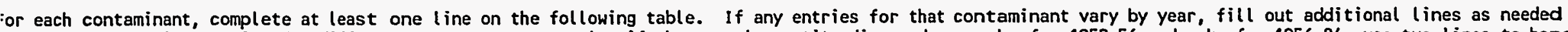

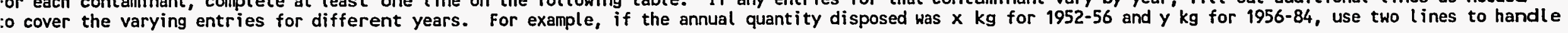
his situation.

\begin{tabular}{|c|c|c|c|c|c|c|c|c|c|c|}
\hline Radionuclide & Physical Form & Chemical Form & $\begin{array}{l}\text { (A)nnual } /(T) \text { otal } \\
\text { Quantity }\end{array}$ & Unit & $\begin{array}{l}\text { Begin } \\
\text { Year }\end{array}$ & $\begin{array}{l}\text { End } \\
\text { Year }\end{array}$ & $\begin{array}{l}\text { Samp } \\
\text { les? }\end{array}$ & $\begin{array}{c}\text { Minimum } \\
\text { Value/\#Samp }\end{array}$ & $\begin{array}{r}\text { Maximum } \\
\text { Value/STO }\end{array}$ & $\begin{array}{l}\text { Basis for } \\
\text { Uncertainty }\end{array}$ \\
\hline Cs-134 & Metallic. & Unknown. & T 1.6370500000000 & $\mathrm{CI}$ & 1991 & 1991 & N & $-20 \%$ & $+20 \%$ & See comment below. \\
\hline $\mathrm{Co}-60$ & Metallic. & Unknown. & T. .00747899490000 & CI & 1992 & 1992 & N & $-20 \%$ & $+20 \%$ & See comment below. \\
\hline Cs-137 & Metallic. & Unknown. & T. .02596676610000 & CI & 1992 & 1992 & N & $-20 \%$ & $+20 \%$ & See comment below. \\
\hline Eu-152 & Metallic. & Unknown. & $T .00000502190000$ & CI & 1992 & 1992 & N & $-20 \%$ & $+20 \%$ & See comment below. \\
\hline Eu-154 & Metallic. & Unknown. & $T .00012808500000$ & CI & 1992 & 1992 & N & $-20 \%$ & $+20 \%$ & See comment below. \\
\hline$S b-125$ & Metallic. & Unknown. & $T .00008800000000$ & CI & 1992 & 1992 & $\mathbf{N}$ & $-20 \%$ & $+20 \%$ & See comment below. \\
\hline Co-58 & Metallic. & Unknown. & $T .00002608500000$ & CI & 1992 & 1992 & N & $-20 \%$ & $+20 \%$ & See comment below. \\
\hline Cs-134 & Metallic. & Unknown. & $T .00039446500000$ & CI & 1992 & 1992 & $\mathbf{N}$ & $-20 \%$ & $+20 \%$ & See comment below. \\
\hline$M n-54$ & Metallic. & Unknown. & $T .00012961500000$ & CI & 1992 & 1992 & $\mathbf{N}$ & $-20 \%$ & $+20 \%$ & See comment below. \\
\hline
\end{tabular}

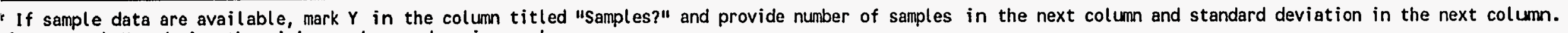
if not, mark $N$ and give the minimum value and maximum value.

ddditional information or explanations (indicate pertinent contaminant)

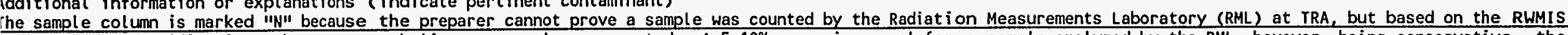

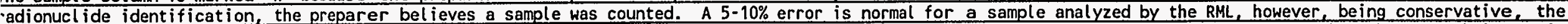

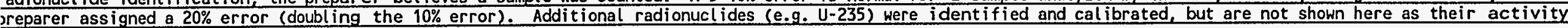
vas less than $1 E-11$ curies. 


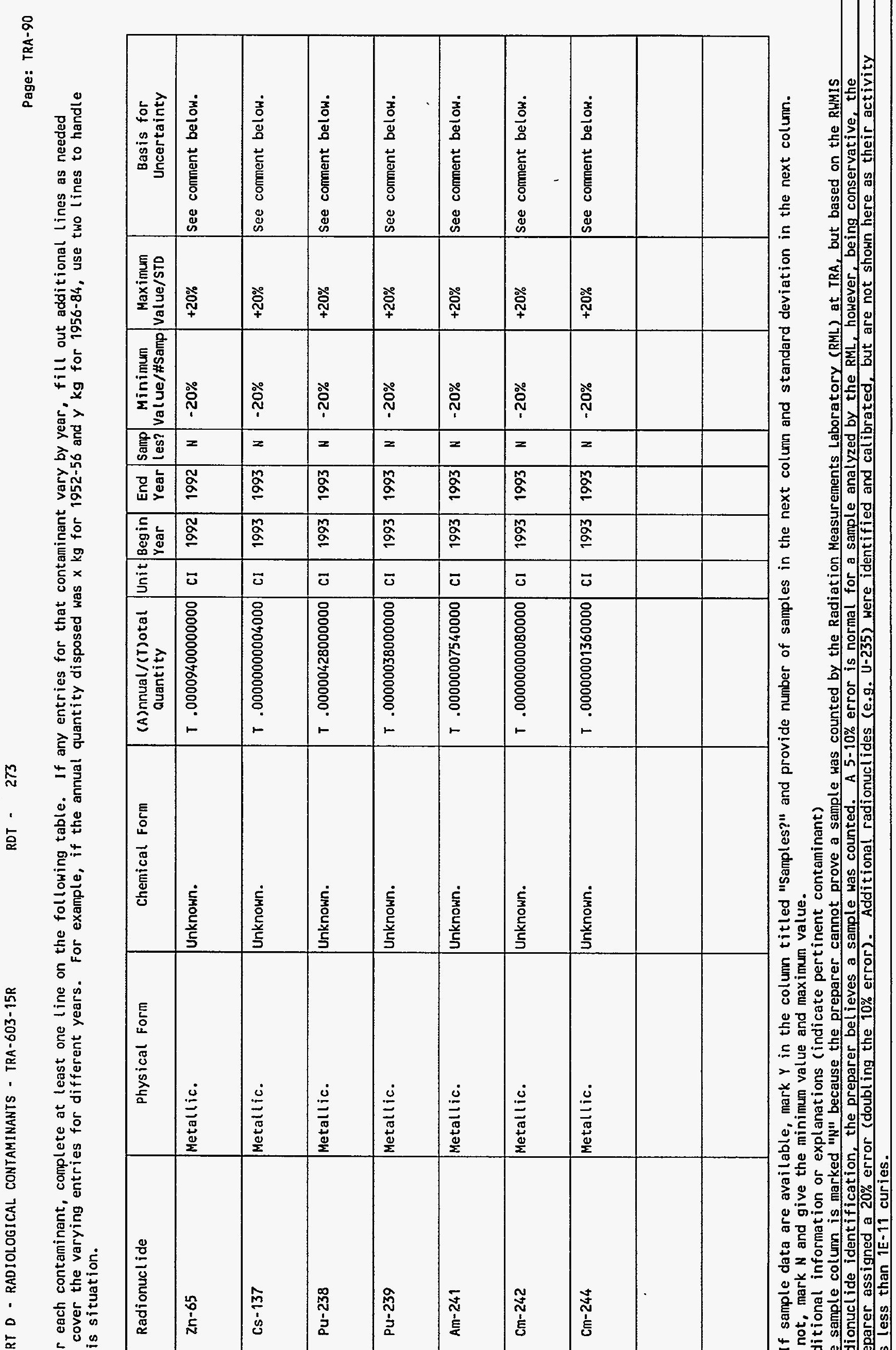


- Type of source of information: check box)

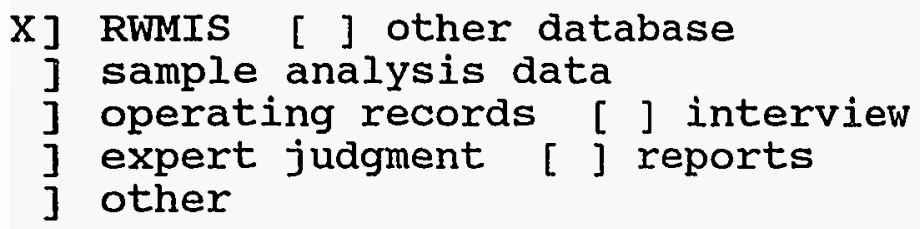

- Do the data conflict with RWMIS? (Historical or Present Data Only) ] no

$\mathrm{x}]$ yes

- Major unknowns in inventories of ontaminants:

AP and MFP were unknown.
2. Details concerning source (names, report no., dates, etc.)

\section{If other than best estimate, explain why:}

6. If yes, explain why:

A portion of or all of the measurements were assumed to be taken using a G-M detector which only measures gamma emitters; beta emitters have been added to the RWMIS activity based on scaling factors. Therefore, the total activity will be greater than that in RWMIS.

8. Key assumptions used to deal with the unknowns: MAP and MFP radionuclides were chosen based on EPR1 NP-5077 for dry active waste for PWRs. PWR was chosen because it is similar to ATR, the reactor at TRA. The radionuclides were chosen based on a combination of the following: cross section, half-lives, and their fission products. 
cause of the form (the various types and shapes) of the material, it is not known whether a ipment's activity was determined with a G-M counter or a NAI detector using smears. Therefore, e true activity and uncertainty is unknown. The G-M counter method has been assumed as the method ed to estimate the gamma emitting radionuclides. Although a G-M counter cannot estimate alpha itting radionuclides, the alpha activities were scaled from the gamma activities. Therefore, the M counter correction applies for the alpha emitters, CIDRA calculated best estimate, upper and wer bounds, accordingly. 
DATA INPUT FOR HISTORICAL DATA TASK FOR RWMC SUBSURFACE DISPOSAL AREA

PART A - GENERAL INFORMATION RDT - 274

1. Preparer: Amaro, C.

2. Date prepared: $07 / 03 / 94$

3. Generator: TRA

(area or contractor - use code from attached list)

5. Number of waste stream from this facility: $18 \mathrm{R}$

7. Type of radioactive waste (check box):

[ ] TRU or suspect TRU

[X] LLW

[ ] non-radioactive

8. Actual years disposed of at SDA: Starting year 1984 Ending year 1986

4. Particular facility: 603

(building number - use code from attached list)

6. Waste stream:

Plant waste which consists of rags, floor sweepings, and glassware. It is compactible.

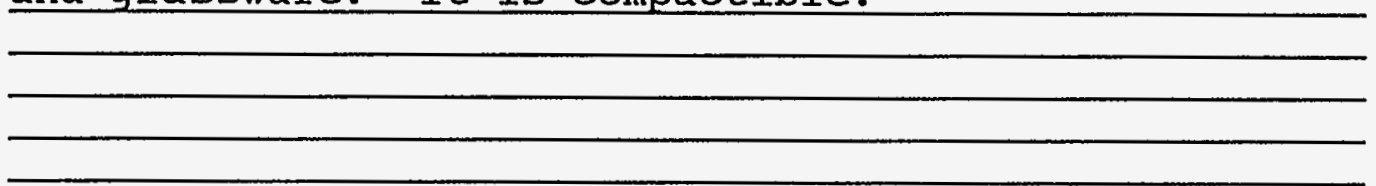

9. Waste stream volume:

Amount 37.2661 Units Cubic meters.

Check box: [ ] annual or $[\mathrm{X}]$ total over all years Check box: [ ] container volume or $[\mathrm{X}]$ waste volume

10. Comments (specify number of pertinent question): 4. Also 604 and 607 . 
- General physical form (see attached list) lass.

X] other (specify)

aper.

- Chemical form:

nknown.

- Waste container type (see attached list) ale.

- Comments (specify number of pertinent question):
2. Details on physical form(particularly confinement related) Consists of rags, floor sweepings, and glassware. It is compactible.

4. Inner packaging: [ ] plastic bag [X] plastic liner

[ ] metal liner [ ] none [ ] other (specify)

6. Other characteristics of interest: 
or each contaminant, complete at least one line on the following table. If any entries for that contaminant vary by year, fill out additional lines as needed

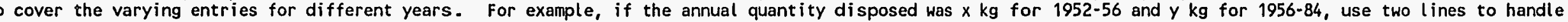
is situation.

\begin{tabular}{|c|c|c|c|c|c|c|c|c|c|c|}
\hline Radionuclide & Physical form & Chemical Form & $\begin{array}{l}\text { (A)nnual / (T)otal } \\
\text { Quant i ty }\end{array}$ & Unit & $\begin{array}{l}\text { Begin } \\
\text { Year }\end{array}$ & $\begin{array}{l}\text { End } \\
\text { Year }\end{array}$ & $\begin{array}{l}\text { Samp } \\
\text { les? }\end{array}$ & $\begin{array}{c}\text { Minimum } \\
\text { Value/\#Samp }\end{array}$ & $\begin{array}{r}\text { Maximum } \\
\text { Value/STD }\end{array}$ & $\begin{array}{l}\text { Basis for } \\
\text { Uncertainty }\end{array}$ \\
\hline $\mathrm{H}-3$ & Unknown. & Unknown. & T. .01208000000000 & $\mathrm{Cl}$ & 1984 & 1984 & N & & & See continuation page. \\
\hline$c-14$ & Unknown. & Unknown. & T.00162100000000 & CI & 1984 & 1984 & N & & & See cont inuation page. \\
\hline $\mathrm{Fe}-55$ & Unknown. & Unknown. & T. .27990000000000 & Cl & 1984 & 1984 & N & & & See continuation page. \\
\hline $\mathrm{Ni}-59$ & Unknown. & Unknown. & T.00008397000000 & CI & 1984 & 1984 & N & & & See continuation page. \\
\hline $\mathrm{Ni}-63$ & Unknown. & Unknown. & T .04714000000000 & CI & 1984 & 1984 & $N$ & & & See continuation page. \\
\hline Co-60 & Unknown. & Unknown. & T. .09870000000000 & $\mathrm{CI}$ & 1984 & 1984 & N & & & See continuation page. \\
\hline $\mathrm{Sr}-90$ & Unknown. & Unknown. & $T .00013550000000$ & CI & 1984 & 1984 & N & & & See continuation page. \\
\hline Tc-99 & Unknown. & Unknown. & T. .00002652000000 & CI & 1984 & 1984 & N & & & See continuation page. \\
\hline I -129 & Unknown. & Unknown. & T. .00000000689000 & CI & 1984 & 1984 & N & & & See continuation page. \\
\hline
\end{tabular}

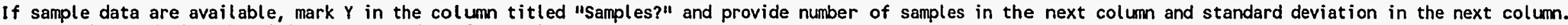
not, mark $N$ and give the minimum value and maximum value.

ditional information or explanations (indicate pertinent contaminant)

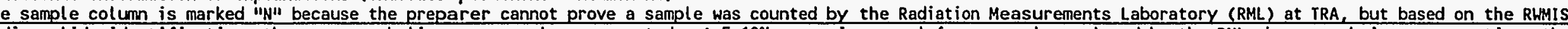

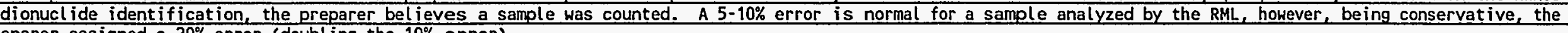
eparer assigned a $20 \%$ error (doubl ing the 10\% error). 
For each contaminant, complete at least one line on the following table. If any entries for that contaminant vary by year, fill out additional lines as needed to cover the varying entries for different years. For example, if the annual quantity disposed was $x \mathrm{~kg}$ for $1952-56$ and y $\mathrm{kg}$ for $1956-84$, use two lines to handle this situation.

\begin{tabular}{|c|c|c|c|c|c|c|c|c|c|c|}
\hline Radionucl ide & Physical form & Chemical Form & $\begin{array}{l}\text { (A)nnual/(T)otal } \\
\text { Quantity }\end{array}$ & Unit & $\begin{array}{l}\text { Begin } \\
\text { Year }\end{array}$ & $\begin{array}{l}\text { End } \\
\text { Year }\end{array}$ & $\begin{array}{l}\text { Samp } \\
\text { Les? }\end{array}$ & $\begin{array}{c}\text { Minimum } \\
\text { value/\#Samp }\end{array}$ & $\begin{array}{r}\text { Maximum } \\
\text { Value/STD }\end{array}$ & $\begin{array}{l}\text { Basis for } \\
\text { Uncertainty }\end{array}$ \\
\hline Cs- 137 & |Unknown. & Unknown. & T. .02946000000000 & $\mathrm{CI}$ & 1984 & 1984 & N & & & See continuation page. \\
\hline $\mathrm{Ce}-144$ & Unknown. & Unknown. & T. .00069240000000 & CI & 1984 & 1984 & N & & & See continuation page. \\
\hline Eu-154 & Unknown. & Unknown. & T. .00000042720000 & CI & 1984 & 1984 & $\mathrm{~N}$ & & & See continuation page. \\
\hline Eu-155 & Unknown. & Unknown. & T. .00138500000000 & $\mathrm{CI}$ & 1984 & 1984 & N & & & See continuation page. \\
\hline$U-234$ & Unknown. & Unknown. & T. .00000030940000 & CI & 1984 & 1984 & N & & & See continuation page. \\
\hline$U-235$ & Unknown. & Unknown. & T. .00000000660000 & CI & 1984 & 1984 & N & & & See continuation page. \\
\hline$U-236$ & Unknown. & Unknown. & T. .00000011790000 & CI & 1984 & 1984 & N & & & See continuation page. \\
\hline Np-237 & Unknown. & Unknown. & Т. .00000019150000 & $\mathrm{CI}$ & 1984 & 1984 & N & & & See continuation page. \\
\hline Pu-238 & Unknown. & Unknown. & T. .00000795500000 & CI & 1984 & 1984 & N & & & See continuation page. \\
\hline
\end{tabular}

*If sample data are available, mark $Y$ in the column titled "Samples?" and provide number of samples in the next column and standard deviation in the next column. If not, mark $N$ and give the minimum value and maximum value.

Additional information or explanations (indicate pertinent contaminant)

The sample column is marked "N" because the preparer cannot prove a sample was counted by the Radiation Measurements Laboratory (RML) at TRA, but based on the RUMIS radionucl ide identification, the preparer believes a sample was counted. A 5-10\% error is normal for a sample analyzed by the RML, however, being conservative, the preparer assigned a $20 \%$ error (doubling the $10 \%$ error). 


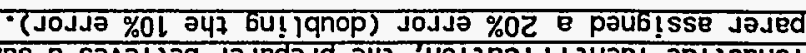

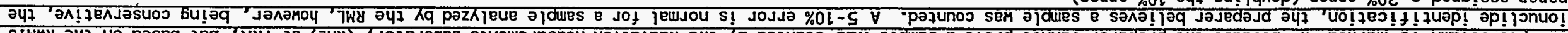

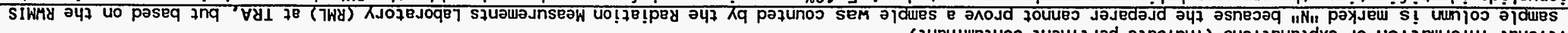

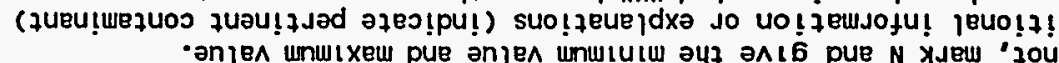

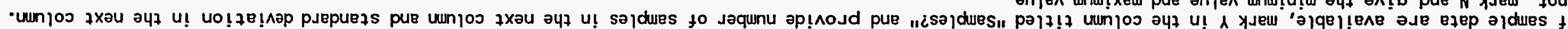

\begin{tabular}{|c|c|c|c|c|c|c|c|c|c|c|}
\hline - abed uo! zenu! zuos әәS & & & N & 5861 & $\$ 861$ & IJ & $00000000698000^{\circ} 1$ & -umouxun & "umouxun & $1+2-n_{d}$ \\
\hline - ə6ed uo!fenu!zuos әәS & & & N & 5861 & $\$ 861$ & 10 & $00005658000000^{\circ} 1$ & -umoùun & "umoùun & $0 b z-n_{d}$ \\
\hline - ə6ed uo!zenu!zuos әәs & & & N & $\$ 861$ & $\$ 861$ & IJ & $00000 \Sigma \$ 6 \angle 00000^{*} 1$ & -umouxun & -umouxun & OEZ-nd \\
\hline - abed uo!zenu!quos әəs & & & N & $\$ 861$ & $\$ 861$ & 15 & $00000 \Omega S 6200000^{-1} 1$ & -umouxun & -umouxun & $8 \varepsilon z-n_{d}$ \\
\hline - abed uo! lenu!łuos aəs & & & N & 5861 & 5861 & IJ & $00005161000000^{\circ} 1$ & -umouxun & - umouxun & $\angle E Z-d_{N}$ \\
\hline 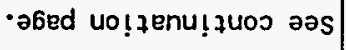 & & & N & $\varsigma 86 \mathrm{l}$ & $\$ 861$ & 13 & $00008 \angle L 1000000^{\circ} 1$ & -umouxun & - UMOUYUत & $9 \varepsilon Z-n$ \\
\hline - abed uo! lenu! дuos aəs & & & N & $\$ 861$ & 5861 & 10 & $00009900000000^{\circ} 1$ & -umouyun & -umouyun & S£Z-n \\
\hline - a6ed uo!łenu!juos әəS & . & & N & $\$ 861$ & $\$ 861$ & 10 & $0000 £ 60 £ 000000^{\circ} 1$ & -umouxun & - umouxun & 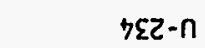 \\
\hline - ə6ed uo!fenu!łuos әəS & & & N & 5861 & $\varsigma 861$ & 13 & $0000000078 \varepsilon 100^{\circ} 1$ & -umouxun & -umouyun & ऽ૬l-n] \\
\hline $\begin{array}{l}\text { Kłu!̣ equaoun } \\
\text { Jot s! seg }\end{array}$ & $\begin{array}{l}\text { als/anjen } \\
\text { unu! } \times \text { Xew }\end{array}$ & $\begin{array}{c}\text { dues\#/anjen } \\
\text { unw!u! }\end{array}$ & $\begin{array}{l}\text { isol } \\
\text { dues }\end{array}$ & $\begin{array}{r}\text { JeOג } \\
\text { pug }\end{array}$ & 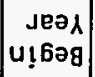 & t!un & $\begin{array}{c}\text { Kł!zueno } \\
\text { j8zo(1)/ןenuu( })\end{array}$ & шЈо与 ןво!шәчว & wsof jeכ!sᄉyd & ap! onuo!pey \\
\hline
\end{tabular}

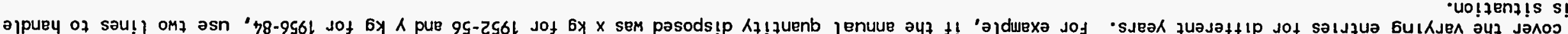

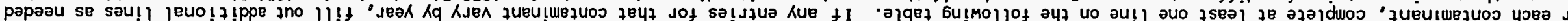

$00 L-\forall Y L: a 6 e d$ 
ir each contaminant, complete at least one line on the following table. If any entries for that contaminant vary by year, fill out additional lines as needed , cover the varying entries for different years. For example, if the annual quantity disposed was $x$ kg for 1952-56 and $y$ kg for $1956-84$, use two lines to handle lis situation.

\begin{tabular}{|c|c|c|c|c|c|c|c|c|c|c|}
\hline Radionuclide & Physical Form & Chemical form & $\begin{array}{l}\text { (A)nnual / (T)otal } \\
\text { Quantity }\end{array}$ & Unit & $\begin{array}{l}\text { Begin } \\
\text { Year }\end{array}$ & $\begin{array}{l}\text { End } \\
\text { Year }\end{array}$ & $\begin{array}{l}\text { Samp } \\
\text { les? }\end{array}$ & $\begin{array}{c}\text { Minimum } \\
\text { Value/\#Samp }\end{array}$ & $\begin{array}{r}\text { Maximum } \\
\text { Value/STD }\end{array}$ & $\begin{array}{l}\text { Basis for } \\
\text { Uncertainty }\end{array}$ \\
\hline$U-236$ & Unknown. & Unknown. & T.00000015140000 & CI & 1986 & 1986 & N & & & See continuation page. \\
\hline$N p-237$ & Unknown. & Unknown. & T. .00000024600000 & CI & 1986 & 1986 & N & & & See cont inuation page. \\
\hline Pu-238 & Unknown. & Unknown. & T.00001022000000 & CI & 1986 & 1986 & N & & & See cont inuation page. \\
\hline Pu-239 & Unknown. & Unknown. & T.00001022000000 & CI & 1986 & 1986 & N & & & See cont inuation page. \\
\hline$P u-240$ & Unknown. & Unknown. & T. .00000107900000 & $\mathrm{CI}$ & 1986 & 1986 & N & & & See continuation page. \\
\hline $\mathrm{Pu}-241$ & Unknown. & Unknown. & T. .00111600000000 & CI & 1986 & 1986 & N & & & See continuation page. \\
\hline Am-241 & Unknown. & Unknown. & T. .00000510900000 & CI & 1986 & 1986 & N & & & See continuation page. \\
\hline$C m-242$ & Unknown. & Unknown. & T.00000510900000 & CI & 1986 & 1986 & N & & & See cont inuation page. \\
\hline $\mathrm{Cm}-244$ & Unknown. & Unknown. & T. .00000473000000 & $\mathrm{CI}$ & 1986 & 1986 & N & & & See cont inuation page. \\
\hline
\end{tabular}

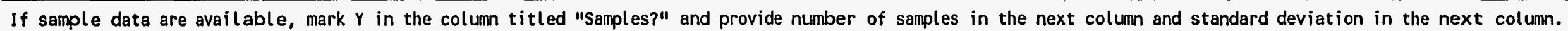
$f$ not, mark $N$ and give the minimum value and maximum value.

dditional information or explanations (indicate pertinent contaminant)

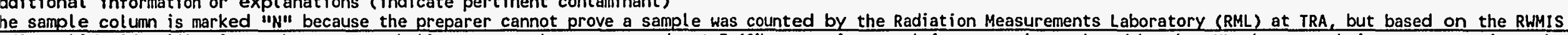

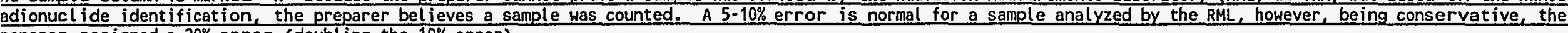
reparer assigned a $20 \%$ error (doubling the $10 \%$ error) 
- Type of source of information: sheck box)

1] RWMIS [ ] other database

] sample analysis data

] operating records [ ] interview

] expert judgment [ ] reports

] other

Do the estimates of contaminant uantities in Part $C$ and $D$ represent: 1] best estimate

] worst case

] other

Do the data conflict with RWMIS? (Historical or Present Data Only) ] no

] yes

Major unknowns in inventories of intaminants:

$P$ and MFP were unknown.
2. Details concerning source (names, report no., dates, etc.)

4. If other than best estimate, explain why:

6. If yes, explain why:

A portion of or all of the measurements were assumed to be taken using a G-M detector which only measures gamma emitters; beta emitters have been added to the RWMIS activity based on scaling factors. Therefore, the total activity will be greater than that in RWMIS.

8. Key assumptions used to deal with the unknowns: MAP and MFP radionuclides were chosen based on EPRI NP-5077 for dry active waste from PWRs. PWR was chosen because it is similar to ATR, the reactor at TRA. The radionuclides were chosen based on a combination of the following: cross section, half-lives, and their fission products. 
3ecause of the form (the various types and shapes) of the material, it is not known whether a shipment's activity was determined with a G-M counter or a NAI detector using smears. Therefore, the true activity and uncertainty is unknown. The G-M counter method has been assumed as the method ised to estimate the gamma emitting radionuclides. Although a G-M counter cannot estimate alpha mitting radionuclides, the alpha activities were scaled from the gamma activities. Therefore, the j-M counter correction applies for the alpha emitters, CIDRA calculated best estimate, and upper and lower bounds, accordingly. 
DATA INPUT FOR HISTORICAL DATA TASK FOR RWMC SUBSURFACE DISPOSAL AREA

ART A - GENERAL INFORMATION RDT - 275

Preparer: Amaro, C.

2. Date prepared: 07/02/94

Generator: TRA

4. Particular facility: 603

area or contractor - use code from attached list)

(building number - use code from attached list)

Number of waste stream from this facility:

6. Waste stream:

Construction materials, concrete, brick, sand, soil and asphalt.

Type of radioactive waste (check box):

] TRU or suspect TRU

ILLW

] non-radioactive

- Actual years disposed of at SDA: carting year 1986 Ending year 1992

9. Waste stream volume:

Amount $\quad 47.0878$ Units Cubic meters.

Check box: [ ] annual or [X] total over all years

Check box: [ ] container volume or [X] waste volume

Comments (specify number of pertinent question):

4. The waste is actually from buildings $635,701,632$, and 661 . 
L. General physical form (see attached list) 2. Details on physical form(particularly confinement related) concrete, brick, and asphalt. $\mathrm{X}]$ other (specify) Material is a combination of concrete, soil, gravel and brick.

3.

3hemical form: Inknown.

4. Inner packaging: [ ] plastic bag [X] plastic liner [ ] metal liner [ ] none [ ] other (specify)

;. Waste container type (see attached 1ist) 6. other characteristics of interest: jooden box.

7. Comments (specify number of pertinent question):

5. Also BLM. 
each contaminant, complete at least one line on the following table. If any entries for that contaminant vary by year, fill out additional lines as needed

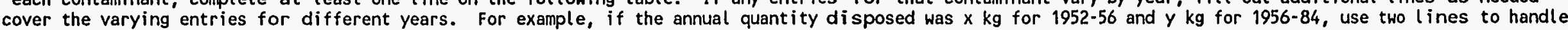
s situation.

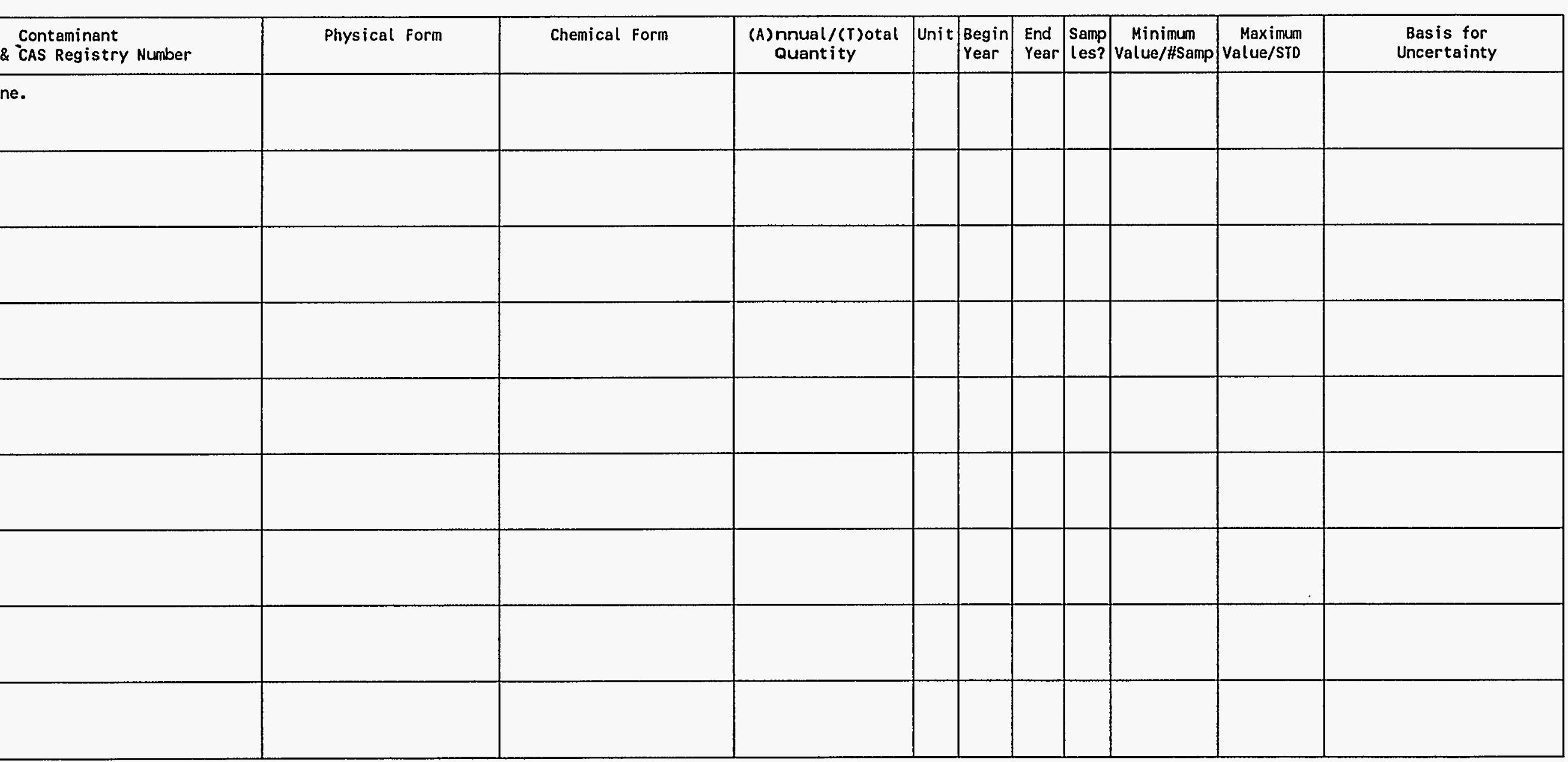

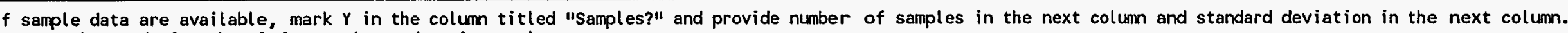
not, mark $N$ and give the minimum value and maximum value.

itional information or explanations (indicate pertinent contaminant) 


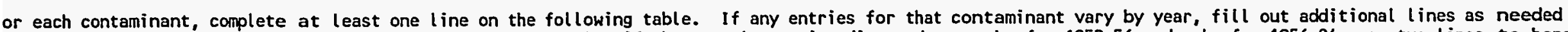

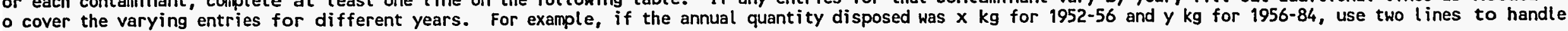
his situation.

\begin{tabular}{|c|c|c|c|c|c|c|c|c|c|c|}
\hline Radionuclide & Physical form & Chemical Form & $\begin{array}{l}\text { (A)nnual/(T)otal } \\
\text { Quantity }\end{array}$ & Unit & $\begin{array}{l}\text { Begin } \\
\text { Year }\end{array}$ & $\begin{array}{l}\text { End } \\
\text { Year }\end{array}$ & $\begin{array}{l}\text { Samp } \\
\text { les? }\end{array}$ & $\begin{array}{c}\text { Minimum } \\
\text { Value/\#Samp }\end{array}$ & $\begin{array}{r}\text { Maximum } \\
\text { Value/STD }\end{array}$ & $\begin{array}{l}\text { Basis for } \\
\text { Uncertainty }\end{array}$ \\
\hline $\mathrm{H}-3$ & Unknown. & Unknown. & T. .05622000000000 & CI & 1986 & 1986 & N & & & See cont inuation page. \\
\hline$c-14$ & Unknown. & Unknown. & $T .00754200000000$ & CI & 1986 & 1986 & N & & & See continuation page. \\
\hline Fe-55 & Unknown. & Unknown. & T 1.3030000000000 & CI & 1986 & 1986 & N & & & See cont inuation page. \\
\hline Ni-59 & Unknown. & Unknown. & $T .00039080000000$ & CI & 1986 & 1986 & $\mathrm{~N}$ & & & See cont inuation page. \\
\hline $\mathrm{Ni}-63$ & Unknown. & Unknown. & $T .21940000000000$ & CI & 1986 & 1986 & N & & & See cont inuation page. \\
\hline Co-60 & Unknown. & Unknown. & $T .45940000000000$ & $\mathrm{Cl}$ & 1986 & 1986 & N & & & See cont inuation page. \\
\hline $\mathrm{Sr}-90$ & Unknown. & Unknown. & $T .00063080000000$ & CI & 1986 & 1986 & N & & & See continuation page. \\
\hline Tc-99 & Unknown. & Unknown. & $T .00012340000000$ & $\mathrm{CI}$ & 1986 & 1986 & $" N$ & & & See cont inuation page. \\
\hline $1-129$ & Unknown. & Unknown. & $r .00000003209000$ & CI & 1986 & 1986 & N & & & See continuation page. \\
\hline
\end{tabular}

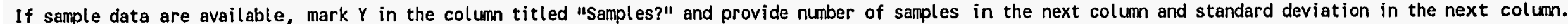
$f$ not, mark $N$ and give the minimum value and maximum value.

dditional information or explanations (indicate pertinent contaminant)

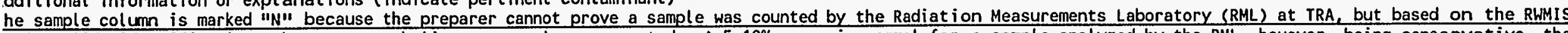

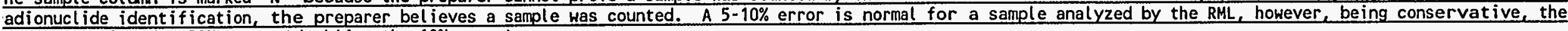
ireparer assigned a $20 \%$ error (doubling the $10 \%$ error). 
each contaminant, complete at least one line on the following table. If any entries for that contaminant vary by year, fill out additional lines as needed

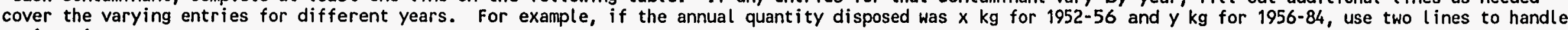
s situation.

\begin{tabular}{|c|c|c|c|c|c|c|c|c|c|c|}
\hline Radionuclide & Physical form & Chemical form & $\begin{array}{l}\text { (A)nnual } /(T) \text { otal } \\
\text { Quant ity }\end{array}$ & Unit & $\begin{array}{l}\text { Begin } \\
\text { Year }\end{array}$ & $\begin{array}{l}\text { End } \\
\text { Year }\end{array}$ & $\begin{array}{l}\text { Samp } \\
\text { les? }\end{array}$ & $\begin{array}{c}\text { Minimum } \\
\text { value/\#Samp }\end{array}$ & $\begin{array}{c}\text { Maximum } \\
\text { Value/STo }\end{array}$ & $\begin{array}{l}\text { Basis for } \\
\text { Uncertainty }\end{array}$ \\
\hline Cs-137 & Unknown. & Unknown. & $T .13710000000000$ & CI & 1986 & 1986 & N & & & See continuation page. \\
\hline $\mathrm{Ce}-144$ & Unknown. & Unknown. & T. .00322200000000 & CI & 1986 & 1986 & N & & & See continuation page. \\
\hline Eu-154 & Unknown. & Unknown. & T.00000198800000 & CI & 1986 & 1986 & N & & & See continuation page. \\
\hline Eu-155 & Unknown. & Unknown. & T. .00644500000000 & $\mathrm{CI}$ & 1986 & 1986 & N & & & See continuation page. \\
\hline$U-234$ & Unknown. & Unknown. & T.00000144000000 & $\mathrm{CI}$ & 1986 & 1986 & $\mathbf{N}$ & & & See cont inuation page. \\
\hline $\mathrm{U}-235$ & Unknown. & Unknown. & $T .00000003090000$ & CI & 1986 & 1986 & ${ }_{2} N$ & & & See continuation page. \\
\hline$U-236$ & Unknown. & Unknown. & $T .00000054850000$ & CI & 1986 & 1986 & $\mathbf{N}$ & & & See cont inuation page. \\
\hline Np-237 & Unknown. & Unknown. & T. .00000089130000 & $\mathrm{CI}$ & 1986 & 1986 & N & & & See continuation page. \\
\hline Pu-238 & Unknown. & Unknown. & T.00003702000000 & $\mathrm{CI}$ & 1986 & 1986 & N & & & See continuation page. \\
\hline
\end{tabular}

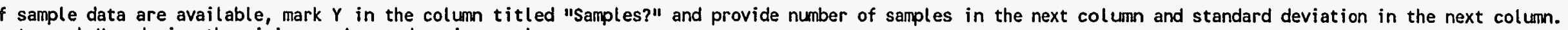
not, mark $N$ and give the minimum value and maximum value.

itional information or explanations (indicate pertinent contaminant)

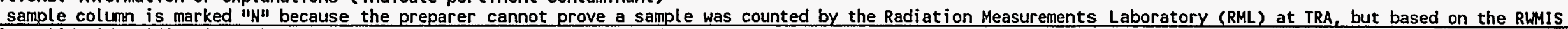

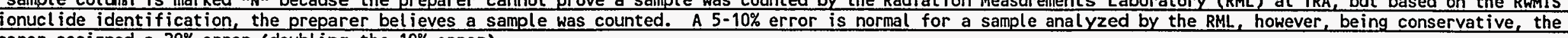
oarer assigned a $20 \%$ error (doubling the 10\% error). 
- Type of source of information: check box)

X] RWMIS [ ] other database

] sample analysis data

] operating records [ ] interview

expert judgment [ ] reports

] other

- Do the estimates of contaminant lantities in Part $C$ and $D$ represent:

1] best estimate

] worst case

] other

- Do the data conflict with RWMIS? (Historical or Present Data Only)
] no

1] yes

Major unknowns in inventories of ontaminants:

IP and MFP were unknown.
2. Details concerning source (names, report no., dates, etc.)

4. If other than best estimate, explain why:

6. If yes, explain why:

A portion of or all of the measurements were assumed to be taken using a G-M detector which only measures gamma emitters; beta emitters have been added to the RWMIS activity based on scaling factors. Therefore, the total activity will be greater than that in RWMIS.

8. Key assumptions used to deal with the unknowns: MAP and MFP radionuclides were chosen based on EPR1 NP-5077 for dry active waste for PWRs. PWR was chosen because it is similar to ATR, the reactor at TRA. The radionuclides were chosen based on a combination of the following: cross section, half-lives, and their fission products. 
secause of the form (the various types and shapes) of the material, it is not known whether a ;hipment's activity was determined with a G-M counter or with a NAI detector using smears. ?herefore, the true activity and uncertainty is unknown. The G-M counter method has been assumed as he method used to estimate the gamma emitting radionuclides. Although a G-M counter cannot stimate alpha emitting radionuclides, the alpha activities were scaled from the gamma activities. herefore, the G-M counter correction applies for the alpha emitters, CIDRA calculated best sstimate, and upper and lower bounds, accordingly. 
DATA INPUT FOR HISTORICAL DATA TASK FOR RWMC SUBSURFACE DISPOSAL AREA

\section{ART A - GENERAL INFORMATION RDT - 278}

Preparer: Amaro, C.

Generator: TRA area or contractor - use code from attached list)

Number of waste stream from this facility: $26 \mathrm{R}$

Type of radioactive waste (check box):

] TRU or suspect TRU

X] LLW

] non-radioactive

Actual years disposed of at SDA: carting year 1987 Ending year 1987
2. Date prepared: 07/03/94

4. Particular facility: 603

(building number - use code from attached list)

6. Waste stream:

Lead.
9. Waste stream volume:

Amount 36.0152 Units Cubic meters.

Check box: [ ] annual or [X] total over all years
Check box: [ ] container volume or [X] waste volume

Comments (specify number of pertinent question): 4. The actual waste is from TRA-B\&W and TRA-ERP. The preparer of this report was unable to determine these facilities. 
General physical form (see attached list) 2. Details on physical form (particularly confinement related) ead.

$\longrightarrow$

\section{Chemical form:}

b metal.

- Waste container type (see attached list) etal box.

- Comments (specify number of pertinent question): 5. Also BXW.
4. Inner packaging: [ ] plastic bag [X] plastic liner [ ] metal liner [ ] none [ ] other (specify)

6. Other characteristics of interest: 
each contaminant, complete at least one line on the following table. If any entries for that contaminant vary by year, fill out additional lines as needed

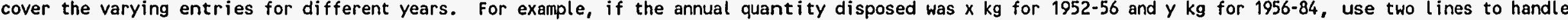
s situation.

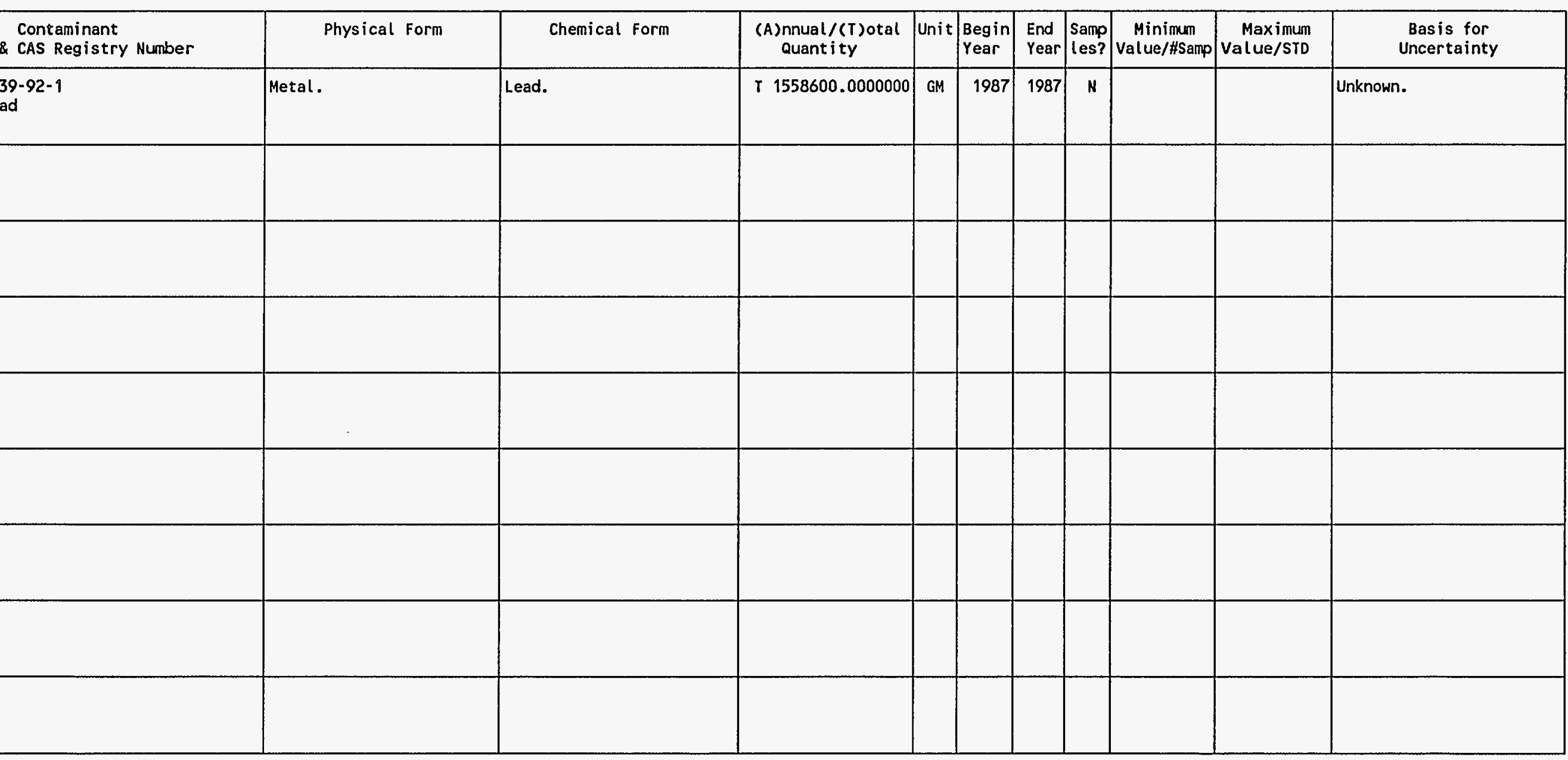

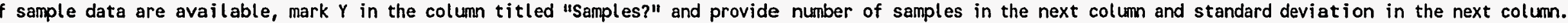
not, mark $N$ and give the minimum value and maximum value.

itional information or explanations (indicate pertinent contaminant) 
or each contaminant, complete at least one line on the following table. If any entries for that contaminant vary by year, fill out additional lines as needed

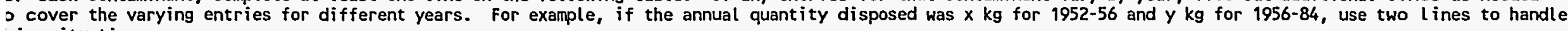
is situation.

\begin{tabular}{|c|c|c|c|c|c|c|c|c|c|c|}
\hline Radionuclide & Physical Form & Chemical form & $\begin{array}{l}\text { (A)nnual } /(T) \text { otal } \\
\text { Quantity }\end{array}$ & Unit & $\begin{array}{l}\text { Begin } \\
\text { Year }\end{array}$ & $\begin{array}{l}\text { End } \\
\text { Year }\end{array}$ & $\begin{array}{l}\text { Samp } \\
\text { les? }\end{array}$ & $\begin{array}{c}\text { Minimum } \\
\text { Value/\#Samp }\end{array}$ & $\begin{array}{r}\text { Maximum } \\
\text { Value/STD }\end{array}$ & $\begin{array}{l}\text { Basis for } \\
\text { Uncertainty }\end{array}$ \\
\hline Co-60 & Metal. & Lead. & $T .00002062600000$ & CI & 1987 & 1987 & N & & & See comment below. \\
\hline Cs -137 & Metal. & Lead. & T. .00001323170000 & CI & 1987 & 1987 & N & & & See comment below. \\
\hline$U-235$ & Metal. & Lead. & $T .00035531090000$ & CI & 1987 & 1987 & N & & & See comment below. \\
\hline & & & & & & & & & & \\
\hline & & & & & & & & & & \\
\hline & & & & & & & & & & \\
\hline & & & & & & & & & & \\
\hline & & & & & & & & & & \\
\hline & & & & & & & & & & \\
\hline & & & & & & & 3 & & & \\
\hline & & & & & & & & & & \\
\hline & & & & & & & & & & \\
\hline & & & & & & & & & & \\
\hline
\end{tabular}

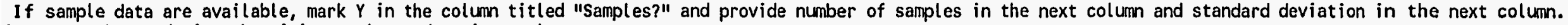
$f$ not, mark $N$ and give the minimum value and maximum value.

dditional information or explanations (indicate pertinent contaminant)

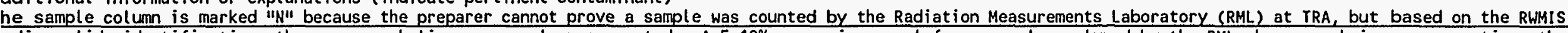

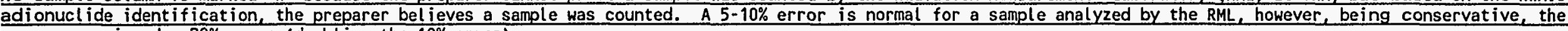
reparer assigned a $20 \%$ error (doubling the $10 \%$ error). 
Type of source of information: heck box)

\section{RWMIS [ ] other database} sample analysis data operating records [ ] interview expert judgment [X] reports other

Do the estimates of contaminant antities in Part $C$ and $D$ represent: ] best estimate worst case

\section{] other}

Do the data conflict with RWMIS? (Historical or Present Data Only) ] no ] yes

Major unknowns in inventories of ntaminants :

$\mathrm{p}$ and MFP were unknown.
2. Details concerning source (names, report no., dates, etc.) A.D. Rogers, Distribution, ADR-8-86 "Elimination of Lead from Low-level Waste", June 24, 1986. A.D. Rogers Distribution, ADR 46-88, "Use of Lead in Low-level. Waste Packages Schedule Exception", December 16, 1987.

4. If other than best estimate, explain why:
8. Key assumptions used to deal with the unknowns: MAP and MFP radionuclides were chosen based on EPR1 NP-5077 for dry active waste for PWRs. PWR was chosen because it is similar to ATR, the reactor at TRA. The radionuclides were chosen based on a combination of the following: cross section, half-lives, and their fission products. 


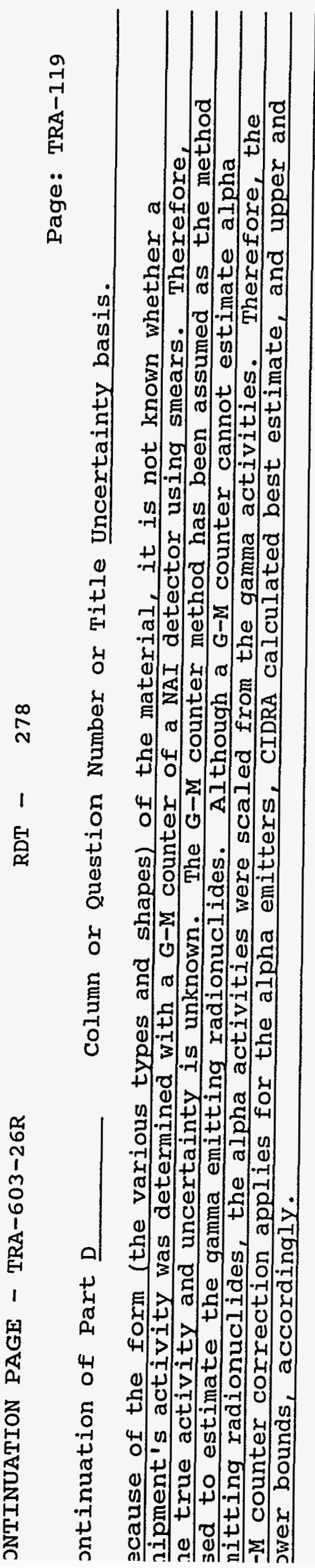


DATA INPUT FOR HISTORICAL DATA TASK FOR RWMC SUBSURFACE DISPOSAL AREA 
$\therefore$ General physical form (see attached list) ilass.

X] other (specify)

letal.

3. Chemical form:

Jnknown.

5. Waste container type (see attached list) rooden box.

7. Comments (specify number of pertinent question): 5. Also BLM, 0 ; the preparer cannot determine what 0 is.
2. Details on physical form (particularly confinement related) This stream is a combination of noncompactible items, such as glass and metal.

4. Inner packaging: [ ] plastic bag [X] plastic liner [ ] metal liner [ ] none [ ] other (specify)

6. Other characteristics of interest: 
each contaminant, complete at least one line on the following table. If any entries for that contaminant vary by year, fill out additional lines as needed

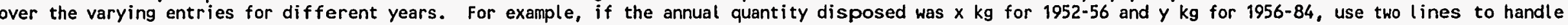
situation.

\begin{tabular}{|c|c|c|c|c|c|c|c|c|c|c|}
\hline ladionucl ide & Physical Form & Chemical form & $\begin{array}{l}\text { (A)nnual/( T r otal } \\
\text { Quantity }\end{array}$ & Unit & $\begin{array}{l}\text { Begin } \\
\text { Year }\end{array}$ & $\begin{array}{l}\text { End } \\
\text { Year }\end{array}$ & $\begin{array}{l}\text { Samp } \\
\text { les? }\end{array}$ & $\begin{array}{c}\text { Minimum } \\
\text { Value/\#Samp }\end{array}$ & $\begin{array}{r}\text { Maximum } \\
\text { Value/STD }\end{array}$ & $\begin{array}{l}\text { Basis for } \\
\text { Uncertainty }\end{array}$ \\
\hline s- 137 & Unknown. & Unknown. & T 2.6540000000000 & CI & 1984 & 1984 & N & & & See continuation page. \\
\hline$e-144$ & Unknown. & Unknown. & $T .06236000000000$ & Cl & 1984 & 1984 & N & & & See continuation page. \\
\hline$u-154$ & Unknown. & Unknown. & $T .00003848000000$ & Cl & 1984 & 1984 & N & & & See continuation page. \\
\hline$u-155$ & Unknown. & Unknown. & T. .12470000000000 & CI & 1984 & 1984 & N & & & See continuation page. \\
\hline-234 & Unknown. & Unknown. & T.00002786000000 & CI & 1984 & 1984 & $\mathbf{N}$ & & & See cont inuation page. \\
\hline-235 & Unknown. & Unknown. & T.00000059710000 & CI & 1984 & 1984 & N & & & See continuation page. \\
\hline $1-236$ & Unknown. & Unknown. & $T .00001062000000$ & CI & 1984 & 1984 & N & & & See cont inuation page. \\
\hline$p-237$ & Unknown. & Unknown. & $T .00001725000000$ & CI & 1984 & 1984 & $\mathrm{~N}$ & & & See cont inuation page. \\
\hline$u-238$ & Unknown. & Unknown. & $T .00071650000000$ & CI & 1984 & 1984 & $\mathrm{~N}$ & & & See cont inuation page. \\
\hline
\end{tabular}

sample data are available, mark $Y$ in the column titled "Samples?" and provide number of samples in the next column and standard deviation in the next column. t, mark $N$ and give the minimum value and maximum value.

:ional information or explanations (indicate pertinent contaminant)

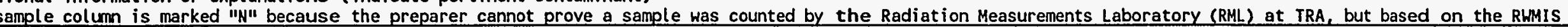

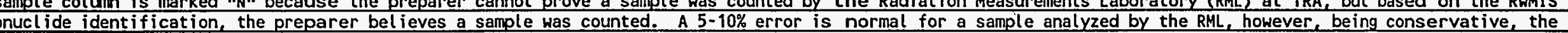
rer assigned a $20 \%$ error (doubl ing the $10 \%$ error). 
ir each contaminant, complete at least one line on the following table. If any entries for that contaminant vary by year, fill out additional lines as needed

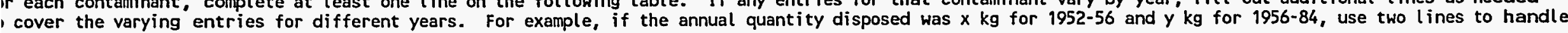
is situation.

\begin{tabular}{|c|c|c|c|c|c|c|c|c|c|c|}
\hline Radionucl ide & Physical form & Chemical Form & $\begin{array}{l}\text { (A)nnual/(T)otal } \\
\text { Quantity }\end{array}$ & Unit & \begin{tabular}{|l|} 
Begin \\
Year
\end{tabular} & $\begin{array}{l}\text { End } \\
\text { Year }\end{array}$ & $\begin{array}{l}\text { Samp } \\
\text { les? }\end{array}$ & $\begin{array}{c}\text { Minimum } \\
\text { Value/\#Samp }\end{array}$ & $\begin{array}{c}\text { Maximum } \\
\text { Value/STD }\end{array}$ & $\begin{array}{l}\text { Basis for } \\
\text { Uncertainty }\end{array}$ \\
\hline Pu-239 & Unknown. & Unknown. & T. .00071650000000 & CI & 1984 & 1984 & N & & & See cont inuation page. \\
\hline Pu-240 & Unknown. & Unknown. & T. .00007563000000 & CI & 1984 & 1984 & N & & & See continuation page. \\
\hline Pu-241 & Unknown. & Unknown. & $T .07829000000000$ & $\mathrm{CI}$ & 1984 & 1984 & N & & & See continuation page. \\
\hline$A m-241$ & Unknown. & Unknown. & $T .00035830000000$ & $\mathrm{Cl}$ & 1984 & 1984 & $N$ & & & See continuation page. \\
\hline $\mathrm{Cm}-242$ & Unknown. & Unknown. & $T .00035830000000$ & CI & 1984 & 1984 & N & & & See continuation page. \\
\hline $\mathrm{Cm}-244$ & Unknown. & Unknown. & $T .00033170000000$ & $\mathrm{Cl}$ & 1984 & 1984 & N & & & See continuation page. \\
\hline$U-235$ & Unknown. & Unknown. & $T .00556000000000$ & $\mathrm{CI}$ & 1984 & 1984 & N & $-20 \%$ & $+20 \%$ & See comment below. \\
\hline$U-238$ & Unknown. & Unknown. & $T .00556000000000$ & $\mathrm{Cl}$ & 1984 & 1984 & N & $-20 \%$ & $+20 \%$ & See comment below. \\
\hline$H-3$ & Unknown. & Unknown. & T 3.1700000000000 & $\mathrm{Cl}$ & 1985 & 1985 & N & & & See continuation page. \\
\hline
\end{tabular}

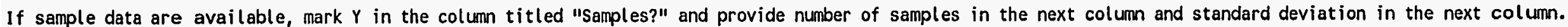
not, mark $N$ and give the minimum value and maximum value.

dditional information or explanations (indicate pertinent contaminant)

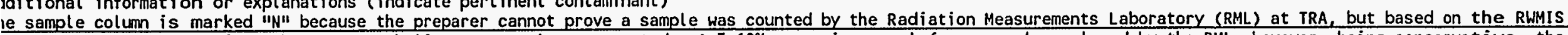

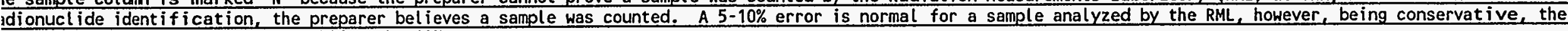
eparer assigned a $20 \%$ error (doubling the $10 \%$ error) 
each contaminant, complete at least one line on the following table. If any entries for that contaminant vary by year, fill out additional lines as needed over the varying entries for different years. For example, if the annual quantity disposed was $x \mathrm{~kg}$ for $1952-56$ and $y \mathrm{~kg}$ for $1956-84$, use two lines to handle situation.

\begin{tabular}{|c|c|c|c|c|c|c|c|c|c|c|}
\hline ?adionucl ide & Physical Form & Chemical Form & $\begin{array}{l}\text { (A)nnual/(T)otal } \\
\text { Quant ity }\end{array}$ & Unit & $\begin{array}{l}\text { Begin } \\
\text { Year }\end{array}$ & $\begin{array}{l}\text { End } \\
\text { Year }\end{array}$ & $\begin{array}{l}\text { Samp } \\
\text { Les? }\end{array}$ & $\begin{array}{c}\text { Minimum } \\
\text { Value/\#Samp }\end{array}$ & $\begin{array}{r}\text { Maximum } \\
\text { Value/STD }\end{array}$ & $\begin{array}{l}\text { Basis for } \\
\text { Uncertainty }\end{array}$ \\
\hline$:-14$ & Unknown. & Unknown. & $T .42530000000000$ & CI & 1985 & 1985 & N & & & See continuation page. \\
\hline$e-55$ & Unknown. & Unknown. & T 73.450000000000 & $\mathrm{Cl}$ & 1985 & 1985 & N & & & See continuation page. \\
\hline$i-59$ & Unknown. & Unknown. & $T .02204000000000$ & $\mathrm{Cl}$ & 1985 & 1985 & N & & & See continuation page. \\
\hline$i-63$ & Unknown. & Unknown. & T 12.370000000000 & $\mathrm{Cl}$ & 1985 & 1985 & N & & & See continuation page. \\
\hline 0.60 & Unknown. & Unknown. & T 25.900000000000 & $\mathrm{Cl}$ & 1985 & 1985 & N & & & See continuation page. \\
\hline$r-90$ & Unknown. & Unknown. & $T .03557000000000$ & $\mathrm{Cl}$ & 1985 & 1985 & N & & & See continuation page. \\
\hline$c-99$ & Unknown. & Unknown. & $T .00695900000000$ & $\mathrm{CI}$ & 1985 & 1985 & N & & & See continuation page. \\
\hline-129 & Unknown. & Unknown. & $\mathrm{T} .00000180900000$ & CI & 1985 & 1985 & N & & & See continuation page. \\
\hline$s-137$ & Unknown. & Unknown. & T $7.7320000000000^{-}$ & $\mathrm{CI}$ & 1985 & 1985 & N & & & See cont inuation page. \\
\hline
\end{tabular}

sample data are available, mark $Y$ in the column titled "Samples?" and provide number of samples in the next column and standard deviation in the next column. t, mark $N$ and give the minimum value and maximum value.

ional information or explanations (indicate pertinent contaminant)

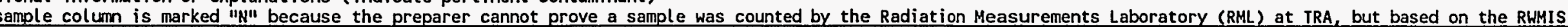

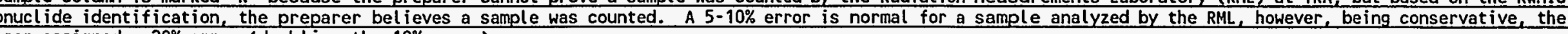
irer assigned a $20 \%$ error (doubling the $10 \%$ error). 
$r$ each contaminant, complete at least one line on the following table. If any entries for that contaminant vary by year, fill out additional lines as needed

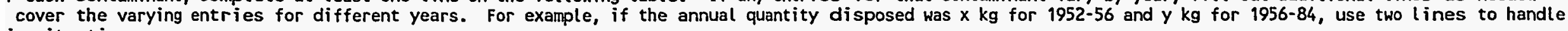
is situation.

\begin{tabular}{|c|c|c|c|c|c|c|c|c|c|c|}
\hline Radionucl ide & Physical form & Chemical Form & $\begin{array}{l}\text { (A)nnual } /(T \text { ) otal } \\
\text { Quant ity }\end{array}$ & Unit & $\begin{array}{l}\text { Begin } \\
\text { Year }\end{array}$ & $\begin{array}{l}\text { End } \\
\text { Year }\end{array}$ & $\begin{array}{l}\text { Samp } \\
\text { les? }\end{array}$ & $\begin{array}{c}\text { Minimum } \\
\text { Value/\#Samp }\end{array}$ & $\begin{array}{r}\text { Maximum } \\
\text { Value/STD }\end{array}$ & $\begin{array}{l}\text { Basis for } \\
\text { Uncertainty }\end{array}$ \\
\hline $\mathrm{Ce}-144$ & Unknown. & Unknown. & T.18170000000000 & CI & 1985 & 1985 & N & & & See continuation page. \\
\hline Eu- 154 & Unknown. & Unknown. & T. .00011210000000 & CI & 1985 & 1985 & N & & & See continuation page. \\
\hline Eu- 155 & Unknown. & Unknown. & T.36340000000000 & CI & 1985 & 1985 & N & & & See continuation page. \\
\hline$U-234$ & Unknown. & Unknown. & T.00008119000000 & $\mathrm{CI}$ & 1985 & 1985 & N & & & See continuation page. \\
\hline$U-235$ & Unknown. & Unknown. & T. .00000174000000 & CI & 1985 & 1985 & N & & & See continuation page. \\
\hline$U-236$ & Unknown. & Unknown. & T.00003093000000 & CI & 1985 & 1985 & N & & & See cont inuation page. \\
\hline$N p-237$ & Unknown. & Unknown. & T. .00005026000000 & CI & 1985 & 1985 & $N$ & & & See continuation page. \\
\hline Pu-238 & Unknown. & Unknown. & T. .00208800000000 & CI & 1985 & 1985 & $\mathbf{N}$ & & & See cont inuation page. \\
\hline Pu-239 & Unknown. & Unknown. & T.00208800000000 & $\mathrm{CI}$ & 1985 & 1985 & N & & & See continuation page. \\
\hline
\end{tabular}

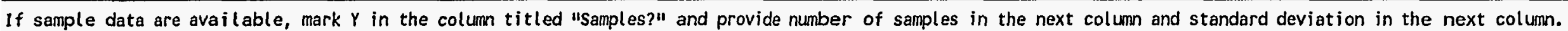
not, mark $N$ and give the minimum value and maximum value.

iditional information or explanations (indicate pertinent contaminant)

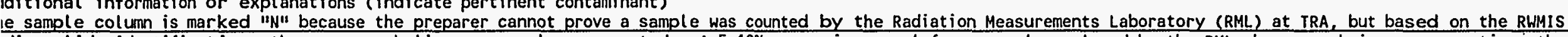

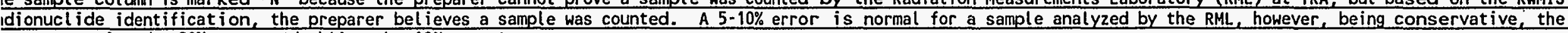
eparer assigned a $20 \%$ error (doubling the $10 \%$ error). 
- each contaminant, complete at least one line on the following table. If any entries for that contaminant vary by year, fill out additional lines as needed

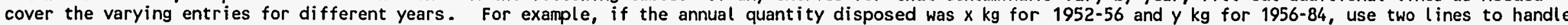
is situation.

\begin{tabular}{|c|c|c|c|c|c|c|c|c|c|c|}
\hline Radionucl ide & Physical form & Chemical form & $\begin{array}{l}\text { (A)nnual } /(T) \text { otal } \\
\text { Quantity }\end{array}$ & Unit & $\begin{array}{l}\text { Begin } \\
\text { Year }\end{array}$ & $\begin{array}{l}\text { End } \\
\text { Year }\end{array}$ & $\begin{array}{l}\text { Samp } \\
\text { les? }\end{array}$ & $\begin{array}{c}\text { Minimum } \\
\text { Value/\#Samp }\end{array}$ & $\begin{array}{r}\text { Maximum } \\
\text { Value/STD }\end{array}$ & $\begin{array}{l}\text { Basis for } \\
\text { Uncertainty }\end{array}$ \\
\hline Pu-240 & Unknown. & Unknown. & $T .00022040000000$ & $\mathrm{CI}$ & 1985 & 1985 & N & & & See continuation page. \\
\hline Pu-241 & Unknown. & Unknown. & $T .22810000000000$ & $\mathrm{Cl}$ & 1985 & 1985 & N & & & See continuation page. \\
\hline$A m-241$ & Unknown. & Unknown. & T. .00104400000000 & $\mathrm{CI}$ & 1985 & 1985 & N & & & See continuation page. \\
\hline $\mathrm{Cm}-242$ & Unknown. & Unknown. & T. .00104400000000 & CI & 1985 & 1985 & $\mathbf{N}$ & & & See cont inuation page. \\
\hline $\mathrm{Cm}-244$ & Unknown. & Unknown. & T.00096650000000 & $\mathrm{CI}$ & 1985 & 1985 & N & & & See continuation page. \\
\hline Co-58 & Unknown. & Unknown. & T. .10000000000000 & $\mathrm{Cl}$ & 1985 & 1985 & N & $-20 \%$ & $+20 \%$ & See comment below. \\
\hline Co- 60 & Unknown. & Unknown. & T 3.4000000000000 & C! & 1985 & 1985 & N & $-20 \%$ & $+20 \%$ & See comment below. \\
\hline Cs -134 & Unknown. & Unknown. & T 1.2900000000000 & CI & 1985 & 1985 & N & $-20 \%$ & $+20 \%$ & See comment below. \\
\hline Cs-137 & Unknown. & Unknown. & T 11.190000000000 & CI & 1985 & 1985 & N & $-20 \%$ & $+20 \%$ & See comment below. \\
\hline
\end{tabular}

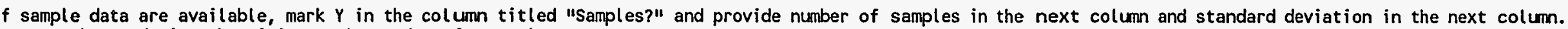
not, mark $\mathrm{N}$ and give the minimum value and maximum value.

itional information or explanations (indicate pertinent contaminant)

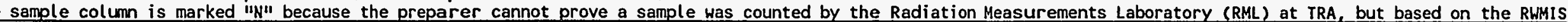

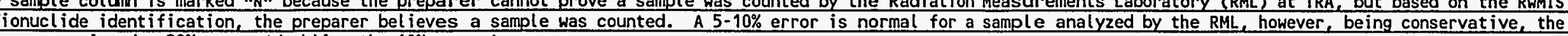
parer assigned a $20 \%$ error (doubling the $10 \%$ error). 
or each contaminant, complete at least one line on the following table. If any entries for that contaminant vary by year, fill out additional lines as needed o cover the varying entries for different years. For example, if the annual quantity disposed was $x \mathrm{~kg}$ for $1952-56$ and $y$ kg for 1956-84, use two lines to handle his situation.

\begin{tabular}{|c|c|c|c|c|c|c|c|c|c|c|}
\hline Radionucl ide & Physical Form & Chemical Form & $\begin{array}{l}\text { (A) nnual/(T)otal } \\
\text { Quantity }\end{array}$ & Unit & $\begin{array}{l}\text { Begin } \\
\text { Year }\end{array}$ & $\begin{array}{l}\text { End } \\
\text { Year }\end{array}$ & $\begin{array}{l}\text { Samp } \\
\text { les? }\end{array}$ & $\begin{array}{c}\text { Minimum } \\
\text { Value/\#Samp }\end{array}$ & $\begin{array}{r}\text { Maximum } \\
\text { Value/STD }\end{array}$ & $\begin{array}{l}\text { Basis for } \\
\text { Uncertainty }\end{array}$ \\
\hline$M n-54$ & Unknown. & Unknown. & $T .05000000000000$ & CI & 1985 & 1985 & N & $-20 \%$ & $+20 \%$ & See comment below. \\
\hline$H-3$ & Unknown. & Unknown. & T. .54120000000000 & CI & 1986 & 1986 & N & & & See continuation page. \\
\hline$c-14$ & Unknown. & Unknown. & T. .07260000000000 & CI & 1986 & 1986 & N & & & See continuation page. \\
\hline $\mathrm{Fe}-55$ & Unknown. & Unknown. & T 12.540000000000 & CI & 1986 & 1986 & N & & & See continuation page. \\
\hline $\mathrm{Ni}-59$ & Unknown. & Unknown. & $r .00376200000000$ & CI & 1986 & 1986 & N & & & See continuation page. \\
\hline $\mathrm{Ni}-63$ & Unknown. & Unknown. & T 2.1120000000000 & $\mathrm{CI}$ & 1986 & 1986 & N & & & See continuation page. \\
\hline Co-60 & Unknown. & Unknown. & T 4.4220000000000 & CI & 1986 & 1986 & N & & & See continuation page. \\
\hline sr-90 & Unknown. & Unknown. & $\tau .00607200000000$ & $\mathrm{CI}$ & 1986 & 1986 & N & & & See continuation page. \\
\hline Tc-99 & Unknown. & |Unknown. & T. .00118800000000 & $\mathrm{CI}$ & 1986 & 1986 & N & & & See continuation page. \\
\hline
\end{tabular}

If sample data are available, mark $Y$ in the column titled "Samples?" and provide number of samples in the next column and standard deviation in the next column. $f$ not, mark $N$ and give the minimum value and maximum value.

dditional information or explanations (indicate pertinent contaminant)

"he sample column is marked "N" because the preparer cannot prove a sample was counted by the Radiation Measurements Laboratory (RML) at TRA, but based on the RHMIS adionuclide identification, the preparer believes a sample was counted. A 5-10\% error is normal for a sample analyzed by the RML, however, being conservative, the reparer assigned a $20 \%$ error (doubling the $10 \%$ error). 
Ir each contaminant, complete at least one line on the following table. If any entries for that contaminant vary by year, fill out additional lines as needed

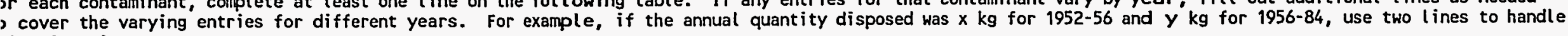
is situation.

\begin{tabular}{|c|c|c|c|c|c|c|c|c|c|c|}
\hline Radionuclide & Physical Form & Chemical Form & $\begin{array}{l}\text { (A)nnual/(T)otal } \\
\text { Quantity }\end{array}$ & Unit & $\begin{array}{l}\text { Begin } \\
\text { Year }\end{array}$ & $\begin{array}{l}\text { End } \\
\text { Year }\end{array}$ & $\begin{array}{l}\text { Samp } \\
\text { les? }\end{array}$ & $\begin{array}{c}\text { Minimum } \\
\text { Value/\#Samp }\end{array}$ & $\begin{array}{r}\text { Maximum } \\
\text { Value/STD }\end{array}$ & $\begin{array}{l}\text { Basis for } \\
\text { Uncertainty }\end{array}$ \\
\hline Pu-238 & Unknown. & Unknown. & T. .00035640000000 & CI & 1986 & 1986 & N & & & See continuation page. \\
\hline Pu-239 & Unknown. & Unknown. & $T .00035640000000$ & $\mathrm{Cl}$ & 1986 & 1986 & N & & & See continuation page. \\
\hline Pu-240 & Unknown. & Unknown. & T. .00003762000000 & $\mathrm{CI}$ & 1986 & 1986 & N & & & See continuation page. \\
\hline Pu-241 & Unknown. & Unknown. & T. .03894000000000 & $\mathrm{CI}$ & 1986 & 1986 & N & & & See continuation page. \\
\hline$A m-241$ & Unknown. & Unknown. & $T .00017820000000$ & $\mathrm{Cl}$ & 1986 & 1986 & N & & & See continuation page. \\
\hline $\mathrm{Cm}-242$ & Unknown. & Unknown. & T.00017820000000 & $\mathrm{CI}$ & 1986 & 1986 & N & & & See continuation page. \\
\hline $\mathrm{Cm}-244$ & Unknown. & Unknown. & T. .00016500000000 & $\mathrm{CI}$ & 1986 & 1986 & N & & & See cont inuation page. \\
\hline & & & & & & & & & & \\
\hline & & & & & & & & & & \\
\hline & & & & & & & & & & \\
\hline
\end{tabular}

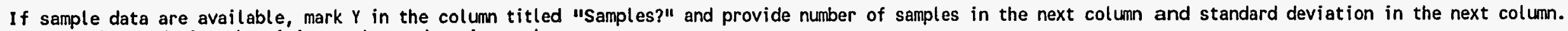
f not, mark $N$ and give the minimum value and maximum value.

dditional information or explanations (indicate pertinent contaminant)

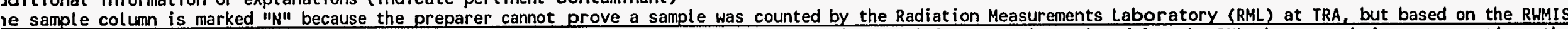

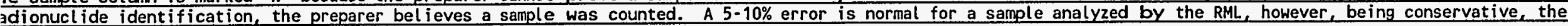
-eparer assigned a $20 \%$ error (doubling the 10\% error). 
Type of source of information: heck box)

\section{RWMIS [ ] other database}

sample analysis data

operating records [ ] interview

expert judgment [X] reports

other

Do the estimates of contaminant lantities in Part $C$ and $D$ represent: ] best estimate

\section{] worst case}

other

Do the data conflict with RWMIS? (Historical or Present Data only) ] no

] yes

Major unknowns in inventories of ntaminants:

$\mathrm{P}$ and MFP were unknown.
2. Details concerning source (names, report no., dates, etc.)

4. If other than best estimate, explain why:

6. If yes, explain why:

A portion of or all of the measurements were assumed to be

taken using a G-M detector which only measures gamma

emitters; beta emitters have been added to the RWMIS

activity based on scaling factors. Therefore, the total activity will be greater than that in RWMIS.

8. Key assumptions used to deal with the unknowns: MAP and MFP radionuclides were chosen based on EPR1 NP-5077 for dry active waste for PWRs. PWR was chosen because it is similar to ATR, the reactor at TRA. The radionuclides were chosen based on a combination of the following: cross section, half-lives, and their fission products. 
ontinuation of Part $\underline{D}$

Column or Question Number or Title Uncertainty basis.

ecause of the form (the various types and shapes) of the material, it is not known whether a hipment's activity was determined with a G-M counter or with a NAI detector using smears.

herefore, the true activity and uncertainty is unknown. The G-M counter method has been assumed as he method used to estimate the gamma emitting radionuclides. Although a G-M counter cannot

stimate alpha emitting radionuclides, the alpha activities were scaled from the gamma activities. herefore, the G-M counter correction applies for the alpha emitters, CIDRA calculated best stimate, and upper and lower bounds, accordingly. 
DATA INPUT FOR HISTORICAL DATA TASK FOR RWMC SUBSURFACE DISPOSAL AREA

\section{RT A - GENERAL INFORMATION RDT - 276}

Preparer: Amaro, C.

Generator: TRA

rea or contractor - use code from attached list)

Number of waste stream from this facility: $\underline{28 \mathrm{R}}$

Type of radioactive waste (check box):

] TRU or suspect TRU

LLW

] non-radioactive

Actual years disposed of at SDA: arting year 1987 Ending year 1989

- Comments (specify number of pertinent question):

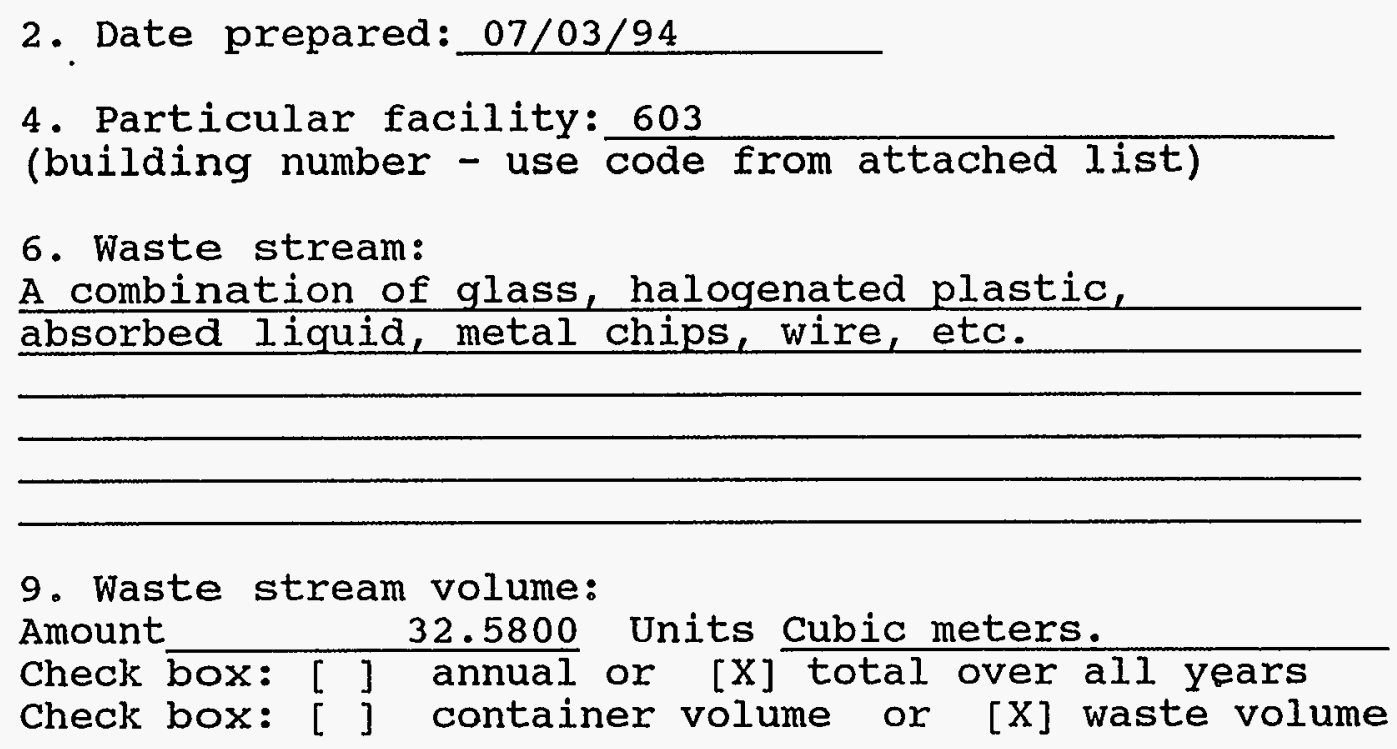

Check box: [ ] annual or $[\mathrm{X}]$ total over all years Check box: [ ] container volume or [X] waste volume 
- General physical form (see attached list) 2. Details on physical form(particularly confinement related)

\section{X] other (specify)} ultiple form.

\section{- Chemical form:} nknown.

- Waste container type (see, attached list) ooden box.

\section{A combination of glass, halogenated plastic, absorbed} liquids, metal chips, wire, etc.

\section{Inner packaging: [ ] plastic bag [X] plastic liner}

[ ] metal liner [] none [ ] other (specify)

6. Other characteristics of interest:

- Comments (specify number of pertinent question): 


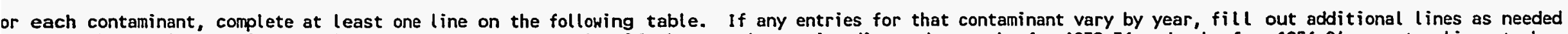

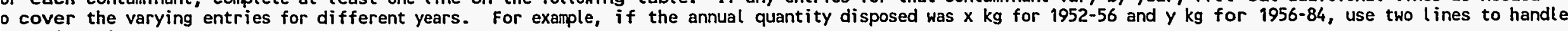
his situation.

\begin{tabular}{|c|c|c|c|c|c|c|c|c|c|c|}
\hline Radionuclide & Physical Form & Chemical Form & $\begin{array}{l}\text { (A)nnual/(T)otal } \\
\text { Quantity }\end{array}$ & Unit & $\begin{array}{l}\text { Begin } \\
\text { Year }\end{array}$ & $\begin{array}{l}\text { End } \\
\text { Year }\end{array}$ & $\begin{array}{l}\text { Samp } \\
\text { les? }\end{array}$ & $\begin{array}{c}\text { Minimum } \\
\text { Value/\#Samp }\end{array}$ & $\begin{array}{r}\text { Maximum } \\
\text { value/STD }\end{array}$ & $\begin{array}{l}\text { Basis for } \\
\text { Uncertainty }\end{array}$ \\
\hline$H-3$ & Unknown. & Unknown. & T. .01866000000000 & Cl & 1987 & 1987 & N & & & See continuation page. \\
\hline$c-14$ & Unknown. & Unknown. & $T .00250200000000$ & CI & 1987 & 1987 & N & & & See continuation page. \\
\hline $\mathrm{Fe}-55$ & Unknown. & Unknown. & T. . 43220000000000 & CI & 1987 & 1987 & N & & & See continuation page. \\
\hline Ni-59 & Unknown. & Unknown. & T. .00012970000000 & CI & 1987 & 1987 & N & & & See continuation page. \\
\hline $\mathrm{Ni}-63$ & Unknown. & Unknown. & $T .07280000000000$ & CI & 1987 & 1987 & N & & & See continuation page. \\
\hline Co- 60 & Unknown. & Unknown. & $T .15240000000000$ & CI & 1987 & 1987 & N & & & See continuation page. \\
\hline $\mathrm{Sr}-90$ & Unknown. & Unknown. & $T .00020930000000$ & $\mathrm{CI}$ & 1987 & 1987 & N & & & See continuation page. \\
\hline Tc-99 & Unknown. & Unknown. & T. .00004095000000 & CI & 1987 & 1987 & N & & & See continuation page. \\
\hline$I-129$ & Unknown. & Jnknown. & $T .00000001065000$ & CI & 1987 & 1987 & N & & & See continuation page. \\
\hline
\end{tabular}

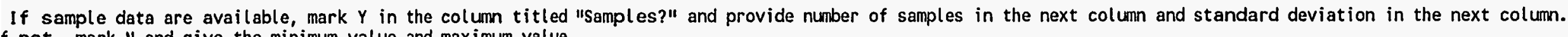
$f$ not, mark $N$ and give the minimum value and maximum value.

dditional information or explanations (indicate pertinent contaminant) 
or each contaminant, complete at least one line on the following table. If any entries for that contaminant vary by year, fill out additional lines as needed

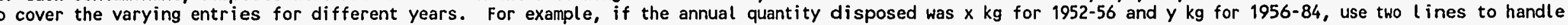
is situation.

\begin{tabular}{|c|c|c|c|c|c|c|c|c|c|c|}
\hline Radionucl ide & Physical Form & Chemical Form & $\begin{array}{l}\text { (A)nnual } /(T) \text { otal } \\
\text { Quant i ty }\end{array}$ & Unit & $\begin{array}{l}\text { Begin } \\
\text { Year }\end{array}$ & $\begin{array}{l}\text { End } \\
\text { Year }\end{array}$ & $\begin{array}{l}\text { Samp } \\
\text { les? }\end{array}$ & $\begin{array}{c}\text { Minimum } \\
\text { Value/\#Samp }\end{array}$ & $\begin{array}{r}\text { Maximum } \\
\text { Value/STD }\end{array}$ & $\begin{array}{l}\text { Basis for } \\
\text { Uncertainty }\end{array}$ \\
\hline Cs -137 & Unknown. & Unknown. & T. .04550000000000 & $\mathrm{Cl}$ & 1987 & 1987 & $\mathbf{N}$ & & & See continuation page. \\
\hline Ce-144 & Unknown. & Unknown. & T. .00106900000000 & $\mathrm{Cl}$ & 1987 & 1987 & $\mathbf{N}$ & & & See continuation page. \\
\hline Eu-154 & Unknown. & Unknown. & T. .00000065980000 & $\mathrm{CI}$ & 1987 & 1987 & $\mathbf{N}$ & & & See continuation page. \\
\hline Eu-155 & Unknown. & Unknown. & T. .00213900000000 & CI & 1987 & 1987 & N & & & See continuation page. \\
\hline$U-234$ & Unknown. & Unknown. & T. .00000047770000 & $\mathrm{CI}$ & 1987 & 1987 & N & & & See continuation page. \\
\hline$U-235$ & Unknown. & Unknown. & T. .00000001020000 & $\mathrm{CI}$ & 1987 & 1987 & N & & & See continuation page. \\
\hline$U-236$ & Unknown. & Unknown. & T. .00000018200000 & $\mathrm{CI}$ & 1987 & 1987 & N & & & See continuation page. \\
\hline Np-237 & Unknown. & Unknown. & T. .00000029580000 & CI & 1987 & 1987 & $\mathbf{N}$ & & & See continuation page. \\
\hline Pu-238 & Unknown. & Unknown. & $T .00001229000000$ & $\mathrm{CI}$ & 1987 & 1987 & $\mathbf{N}$ & & & See continuation page. \\
\hline
\end{tabular}

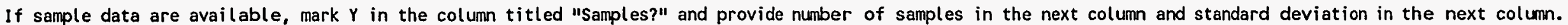
not, mark $N$ and give the minimum value and maximum value.

iditional information or explanations (indicate pertinent contaminant) 
each contaminant, complete at least one line on the following table. If any entries for that contaminant vary by year, fill out additional lines as needed

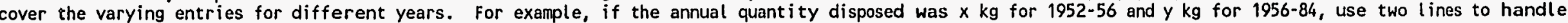
s situation.

\begin{tabular}{|c|c|c|c|c|c|c|c|c|c|c|}
\hline Radionuclide & Physical Form & Chemical Form & $\begin{array}{l}\text { (A)nnual/(T)otal } \\
\text { Quantity }\end{array}$ & Unit & $\begin{array}{l}\text { Begin } \\
\text { Year }\end{array}$ & $\begin{array}{l}\text { End } \\
\text { Year }\end{array}$ & $\begin{array}{l}\text { Samp } \\
\text { les? }\end{array}$ & $\begin{array}{c}\text { Minimum } \\
\text { Value/\#Samp }\end{array}$ & $\begin{array}{l}\text { Maximum } \\
\text { Value/STD }\end{array}$ & $\begin{array}{l}\text { Basis for } \\
\text { Uncertainty }\end{array}$ \\
\hline Ni -59 & Unknown. & Unknown. & T. .00022300000000 & CI & 1989 & 1989 & $\mathbf{N}$ & & & See continuation page. \\
\hline $\mathrm{Ni}-63$ & Unknown. & Unknown. & $r .12520000000000$ & CI & 1989 & 1989 & N & & & See continuation page. \\
\hline Co-60 & Unknown. & Unknown. & $T .26210000000000$ & CI & 1989 & 1989 & N & & & See continuation page. \\
\hline $\mathrm{Sr}-90$ & Unknown. & Unknown. & T. .00035990000000 & CI & 1989 & 1989 & $\mathbf{N}$ & & & See continuation page. \\
\hline Tc-99 & Unknown. & Unknown. & $\tau .00007042000000$ & CI & 1989 & 1989 & $\mathbf{N}$ & & & See continuation page. \\
\hline $1-129$ & Unknown. & Unknown. & $T .00000001831000$ & CI & 1989 & 1989 & N & & & See continuation page. \\
\hline Cs -137 & Unknown. & Unknown. & $T .07824000000000$ & $\mathrm{Cl}$ & 1989 & 1989 & N & & & See continuation page. \\
\hline $\mathrm{Ce}-144$ & Unknown. & Unknown. & $T .00183900000000$ & CI & 1989 & 1989 & $\mathrm{~N}$ & & & See continuation page. \\
\hline Eu-154 & Unknown. & Unknown. & $T .00000113400000$ & CI & 1989 & 1989 & N & & & See continuation page. \\
\hline
\end{tabular}

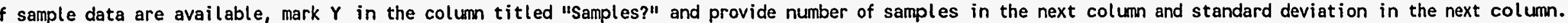
not, mark $N$ and give the minimum value and maximum value.

itional information or explanations (indicate pertinent contaminant) 
r each contaminant, complete at least one line on the following table. If any entries for that contaminant vary by year, fill out additional lines as needed

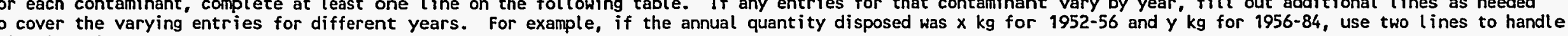
is situation.

\begin{tabular}{|c|c|c|c|c|c|c|c|c|c|c|}
\hline Radionuctide & Physical Form & Chemical Form & $\begin{array}{l}\text { (A)nnual/(T)otal } \\
\text { Quantity }\end{array}$ & Unit & $\begin{array}{l}\text { Begin } \\
\text { Year }\end{array}$ & $\begin{array}{l}\text { End } \\
\text { Year }\end{array}$ & $\begin{array}{l}\text { Samp } \\
\text { les? }\end{array}$ & \begin{tabular}{|} 
Minimum \\
Value/\#Samp
\end{tabular} & $\begin{array}{r}\text { Maximum } \\
\text { Value/STD }\end{array}$ & $\begin{array}{l}\text { Basis for } \\
\text { Uncertainty }\end{array}$ \\
\hline Eu-155 & Unknown. & Unknown. & T. .00367700000000 & Cl & 1989 & 1989 & N & & & See continuation page. \\
\hline$U-234$ & Unknown. & Unknown. & $T .00000082150000$ & CI & 1989 & 1989 & N & & & See continuation page. \\
\hline$U-235$ & Unknown. & Unknown. & T. .00000001760000 & CI & 1989 & 1989 & N & & & See continuation page. \\
\hline$U-236$ & Unknown. & Unknown. & T. .00000031300000 & CI & 1989 & 1989 & N & & & See continuation page. \\
\hline Np-237 & Unknown. & Unknown. & T. .00000050860000 & CI & 1989 & 1989 & N & & & See continuation page. \\
\hline Pu-238 & Unknown. & Unknown. & $T .00002112000000$ & CI & 1989 & 1989 & N & & & See continuation page. \\
\hline Pu-239 & Unknown. & Unknown. & T. .00002112000000 & CI & 1989 & 1989 & N & & & See continuation page. \\
\hline Pu-240 & Unknown. & Unknown. & T. .00000223000000 & CI & 1989 & 1989 & N & & & See continuation page. \\
\hline Pu-241 & Unknown. & Unknown. & $T .00230800000000$ & CI & 1989 & 1989 & N & & & See continuation page. \\
\hline
\end{tabular}

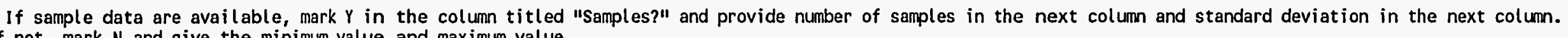
not, mark $N$ and give the minimum value and maximum value.

iditional information or explanations (indicate pertinent contaminant) 
1. Type of source of information: (check box)

[X] RWMIS [ ] other database

] sample analysis data

] operating records [ ] interview

] expert judgment [ ] reports

] other

3. Do the estimates of contaminant quantities in Part $C$ and $D$ represent:

[X] best estimate

] worst case

] other

5. Do the data conflict with RWMIS? (Historical or Present Data only)

] no

[X] yes

7. Major unknowns in inventories of contaminants:

MAP and MFP were unknown.
2. Details concerning source (names, report no., dates, etc.)

4. If other than best estimate, explain why:

6. If yes, explain why:

A portion of or all of the measurements were assumed to be taken using a G-M detector which only measures gamma emitters; beta emitters have been added to the RWMIS activity based on scaling factors. Therefore, the total activity will be greater than that in RWMIS.

8. Key assumptions used to deal with the unknowns: MAP and MFP radionuclides were chosen based on EPR1 NP-5077 for dry active waste for PWRs. PWR was chosen because it is similar to ATR, the reactor at TRA. The radionuclides were chosen based on a combination of the following: cross section, half-lives, and their fission products. 
intinuation of Part $\underline{D}$

Column or Question Number or Title Uncertainty basis.

cause of the form (the various types and shapes) of the material, it is not known whether a ipment's activity was determined with a G-M counter or with a NAI detector using smears. ierefore, the activity and uncertainty is unknown. The G-M counter method has been assumed as the thod used to estimate the gamma emitting radionuclides. Although a G-M counter cannot estimate

pha emitting radionuclides, the alpha activities were scaled from the gamma activities. erefore, the G-M counter correction applies for the alpha emitters, CIDRA calculated best timate, and upper and lower bounds, accordingly. 
DATA INPUT FOR HISTORICAL DATA TASK FOR RWMC SUBSURFACE DISPOSAL AREA

?ART A - GENERAL INFORMATION RDT - 269

L. Preparer: Amaro, C.

3. Generator: TRA (area or contractor - use code from attached list)

5. Number of waste stream from this facility: $2 \mathrm{R}$

7. Type of radioactive waste (check box):

- ] TRU or suspect TRU

X] LLW

] non-radioactive

3. Actual years disposed of at SDA: jtarting year 1984 Ending year 1986

LO. Comments (specify number of pertinent question):
2. Date prepared: $07 / 03 / 94$

4. Particular facility: 632

(building number - use code from attached list)

6. Waste stream:

Hot cell waste consists of small amounts of metal glassware, plastic bottles, anti-c's, etc.

\section{Waste stream volume:}

Amount 0.5684 Units Cubic meters.

Check box: [ ] annual or [X] total over all years Check box: [ ] container volume or [X] waste volume 


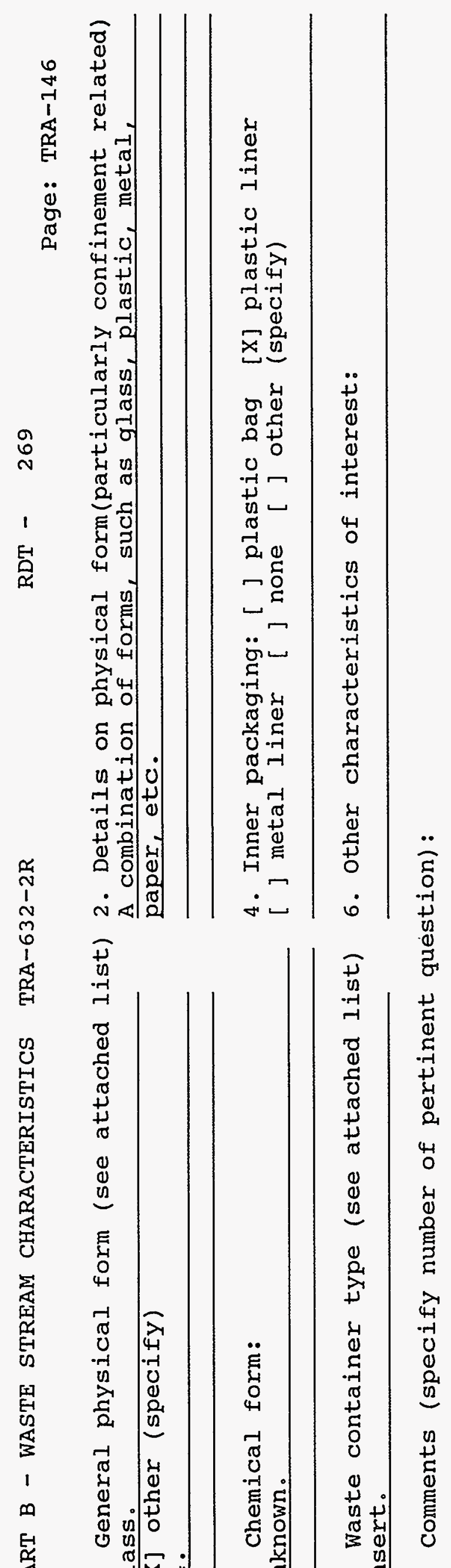


or each contaminant, complete at least one line on the following table. If any entries for that contaminant vary by year, fill out additional lines as needed o cover the varying entries for different years. For example, if the annual quantity disposed was $x$ kg for 1952-56 and $y$ kg for $1956-84$, use two lines to handle his situation.

\begin{tabular}{|c|c|c|c|c|c|c|c|c|c|c|}
\hline $\begin{array}{l}\text { Contaminant } \\
\text { \& CAS Registry Number }\end{array}$ & Physical form & Chemical Form & $\begin{array}{l}\text { (A)nnual/(T)otal } \\
\text { Quantity }\end{array}$ & Unit & $\begin{array}{l}\text { Begin } \\
\text { Year }\end{array}$ & $\begin{array}{l}\text { End } \\
\text { Year }\end{array}$ & $\begin{array}{l}\text { Samp } \\
\text { les? }\end{array}$ & \begin{tabular}{|c|} 
Minimum \\
Value/\#Samp
\end{tabular} & $\begin{array}{r}\text { Maximum } \\
\text { Value/STD }\end{array}$ & $\begin{array}{l}\text { Basis for } \\
\text { Uncertainty }\end{array}$ \\
\hline \multicolumn{11}{|l|}{ Jone. } \\
\hline & & & & & & & & & & \\
\hline & & & & & & & & & & \\
\hline & & & & & & & & & & \\
\hline & & & & & & & & & & \\
\hline & & & & & & & & & & \\
\hline & & & & & & & & & & \\
\hline & & & & & & & & & & \\
\hline & & & & & & & & & & \\
\hline & & & & & & & & & & \\
\hline & & & & & & & & & & \\
\hline & & & & & & & & & & \\
\hline & & & & & & & - & & & \\
\hline & & & & & & & & & & \\
\hline & & & & & & & & & & \\
\hline & & & & & & & & & & \\
\hline
\end{tabular}

If sample data are available, mark $Y$ in the column titled "Samples?" and provide number of samples in the next column and standard deviation in the next column. not, mark $N$ and give the minimum value and maximum value.

ditional information or explanations (indicate pertinent contaminant) 
each contaminant, complete at least one line on the following table. If any entries for that contaminant vary by year, fill out additional lines as needed

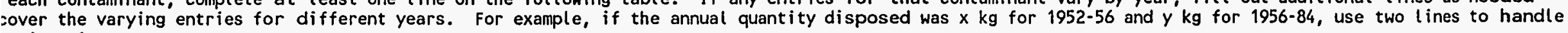
s situation.

\begin{tabular}{|c|c|c|c|c|c|c|c|c|c|c|}
\hline Radionuclide & Physical Form & Chemical form & $\begin{array}{l}\text { (A)nnual/(T) otal } \\
\text { Quantity }\end{array}$ & Unit & $\begin{array}{l}\text { Begin } \\
\text { Year }\end{array}$ & $\begin{array}{l}\text { End } \\
\text { Year }\end{array}$ & $\begin{array}{l}\text { Samp } \\
\text { les? }\end{array}$ & $\begin{array}{c}\text { Minimum } \\
\text { Value/\#Samp }\end{array}$ & $\begin{array}{c}\text { Maximum } \\
\text { Value/STD }\end{array}$ & $\begin{array}{l}\text { Basis for } \\
\text { Uncertainty }\end{array}$ \\
\hline$H-3$ & Unknown. & Unknown. & T 1.2280000000000 & CI & 1984 & 1984 & $N$ & & & See continuation page. \\
\hline C- 14 & Unknown. & Unknown. & $T .16470000000000$ & $\mathrm{CI}$ & 1984 & 1984 & N & & & See continuation page. \\
\hline $\mathrm{Fe}-55$ & Unknown. & Unknown. & T 28.440000000000 & $\mathrm{CI}$ & 1984 & 1984 & N & & & See continuation page. \\
\hline $\mathrm{Ni}-59$ & Unknown. & Unknown. & T. .00853300000000 & CI & 1984 & 1984 & N & & & See continuation page. \\
\hline $\mathrm{Ni}-63$ & Unknown. & Unknown. & T 4.7900000000000 & $\mathrm{CI}$ & 1984 & 1984 & $N$ & & & See continuation page. \\
\hline Co- 60 & Unknown. & Unknown. & T 10.030000000000 & CI & 1984 & 1984 & N & & & See continuation page. \\
\hline $\mathrm{Sr}-90$ & Unknown. & Unknown. & T. .01377000000000 & CI & 1984 & 1984 & N & & & See continuat ion page. \\
\hline Tc-99 & Unknown. & Unknown. & $T .00269500000000$ & $\mathrm{Cl}$ & 1984 & 1984 & N & & & See continuation page. \\
\hline $1-129$ & Unknown. & Unknown. & $T .00000070056000$ & $\mathrm{CI}$ & 1984 & 1984 & $\mathrm{~N}$ & & & See continuation page. \\
\hline
\end{tabular}

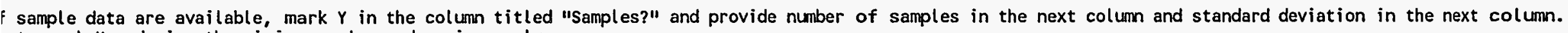
ot, mark $N$ and give the minimum value and maximum value.

itional information or explanations (indicate pertinent contaminant)

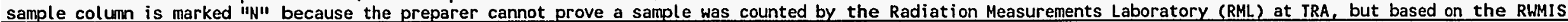

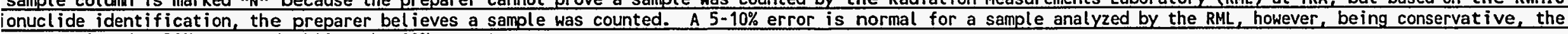
Jarer assigned a $20 \%$ error (doubling the $10 \%$ error). 
or each contaminant, complete at least one line on the following table. If any entries for that contaminant vary by year, fill out additional lines as needed to cover the varying entries for different years. For example, if the annual quantity disposed was $x \mathrm{~kg}$ for $1952-56$ and y $\mathrm{kg}$ for 1956-84, use two lines to handle his situation.

\begin{tabular}{|c|c|c|c|c|c|c|c|c|c|c|}
\hline Radionucl ide & Physical Form & Chemical form & $\begin{array}{l}\text { (A)nnual/(T)otal } \\
\text { Quantity }\end{array}$ & Unit & $\begin{array}{l}\text { Begin } \\
\text { Year }\end{array}$ & $\begin{array}{l}\text { End } \\
\text { Year }\end{array}$ & $\begin{array}{l}\text { Samp } \\
\text { les? }\end{array}$ & $\begin{array}{c}\text { Minimum } \\
\text { Value/\#Samp }\end{array}$ & $\begin{array}{r}\text { Maximum } \\
\text { Value/STD }\end{array}$ & $\begin{array}{l}\text { Basis for } \\
\text { Uncertainty }\end{array}$ \\
\hline Cs-137 & Unknown. & Unknown. & T 2.9940000000000 & $\mathrm{CI}$ & 1984 & 1984 & N & & & See continuation page. \\
\hline $\mathrm{Ce}-144$ & Unknown. & Unknown. & T. .07036000000000 & CI & 1984 & 1984 & N & & & See continuation page. \\
\hline Eu-154 & Unknown. & Unknown. & T. .00004341000000 & CI & 1984 & 1984 & N & & & See continuation page. \\
\hline Eu-155 & Unknown. & Unknown. & T. .14070000000000 & CI & 1984 & 1984 & N & & & See continuation page. \\
\hline$U-234$ & Unknown. & Unknown. & $T .00003144000000$ & Cl & 1984 & 1984 & N & & & See continuation page. \\
\hline$U-235$ & Unknown. & Unknown. & T. .00000067360000 & $\mathrm{CI}$ & 1984 & 1984 & N & & & See continuation page. \\
\hline$U-236$ & Unknown. & Unknown. & T. .00001198000000 & CI & 1984 & 1984 & N & & & See continuation page. \\
\hline Np-237 & Unknown. & Unknown. & T. .00001946000000 & CI & 1984 & 1984 & N & & & See continuation page. \\
\hline Pu-238 & Unknown. & Unknown. & $\mathrm{T} .00080840000000$ & CI & 1984 & 1984 & N & & & See continuation page. \\
\hline
\end{tabular}

If sample data are available, mark $Y$ in the column titled "Samples?" and provide number of samples in the next column and standard deviation in the next column. $f$ not, mark $N$ and give the minimum value and maximum value.

dditional information or explanations (indicate pertinent contaminant)

he sample column is marked "N" because the preparer cannot prove a sample was counted by the Radiation Measurements Laboratory (RML) at TRA, but based on the RUMIS adionucl ide identification, the preparer believes a sample was counted. A 5-10\% error is normal for a sample analyzed by the RML, however, being conservative, the reparer assigned a $20 \%$ error (doubling the $10 \%$ error). 
or each contaminant, complete at least one line on the following table. If any entries for that contaminant vary by year, fill out additional lines as needed

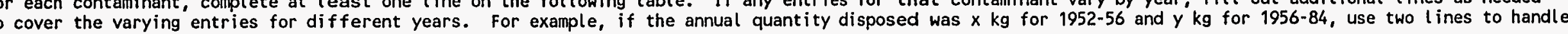
is situation.

\begin{tabular}{|c|c|c|c|c|c|c|c|c|c|c|}
\hline Radionucl ide & Physical Form & Chemical Form & $\begin{array}{l}\text { (A)nnual/(T)otal } \\
\text { quantity }\end{array}$ & Unit & $\begin{array}{l}\text { Begin } \\
\text { Year }\end{array}$ & $\begin{array}{l}\text { End } \\
\text { Year }\end{array}$ & $\begin{array}{l}\text { Samp } \\
\text { les? }\end{array}$ & $\begin{array}{c}\text { Minimum } \\
\text { Value/\#Samp }\end{array}$ & $\begin{array}{r}\text { Maximum } \\
\text { Value/STD }\end{array}$ & $\begin{array}{l}\text { Basis for } \\
\text { Uncertainty }\end{array}$ \\
\hline Pu-239 & Unknown. & Unknown. & T. .00080840000000 & CI & 1984 & 1984 & N & & & See continuation page. \\
\hline Pu-240 & Unknown. & Unknown. & T. .00008533000000 & CI & 1984 & 1984 & N & & & See cont inuation page. \\
\hline Pu-241 & Unknown. & Unknown. & $r .08832000000000$ & $\mathrm{CI}$ & 1984 & 1984 & $N$ & & & See cont inuation page. \\
\hline Am-241 & Unknown. & Unknown. & $T .00040420000000$ & CI & 1984 & 1984 & N & & & See continuation page. \\
\hline $\mathrm{Cm}-242$ & Unknown. & Unknown. & $T .00040420000000$ & CI & 1984 & 1984 & $\because N$ & & & See continuation page. \\
\hline $\mathrm{Cm}-244$ & Unknown. & Unknown. & $T .00037430000000$ & CI & 1984 & 1984 & N & & & See continuation page. \\
\hline $\mathrm{Ce}-144$ & Unknown. & Unknown. & T 10.280000000000 & CI & 1984 & 1984 & N & $-20 \%$ & $+20 \%$ & See comment below. \\
\hline Co- 60 & Unknown. & Unknown. & T 131.75000000000 & $\mathrm{CI}$ & 1984 & 1984 & N & $-20 \%$ & $+20 \%$ & See comment below. \\
\hline Cs -134 & Unknown. & Unknown. & T 19.880000000000 & CI & 1984 & 1984 & N & $-20 \%$ & $+20 \%$ & See comment below. \\
\hline
\end{tabular}

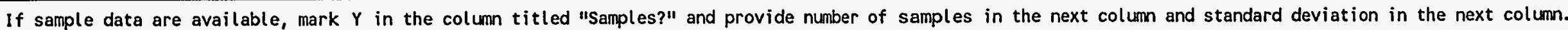
not, mark $N$ and give the minimum value and maximum value.

ditional information or explanations (indicate pertinent contaminant)

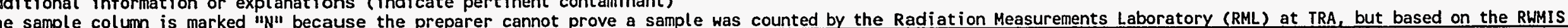

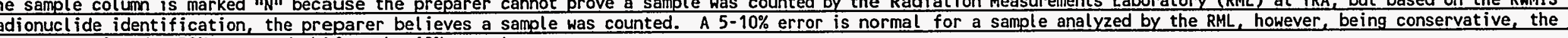
eparer assigned a $20 \%$ error (doubl ing the $10 \%$ error). 
each contaminant, complete at least one line on the following table. If any entries for that contaminant vary by year, fill out additional lines as needed

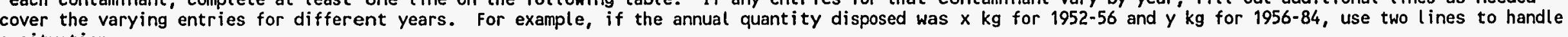
s situation.

\begin{tabular}{|c|c|c|c|c|c|c|c|c|c|c|}
\hline Radionuclide & Physical Form & Chemical Form & $\begin{array}{l}\text { (A)nnual/(T)otal } \\
\text { Quant ity }\end{array}$ & Unit & $\begin{array}{l}\text { Begin } \\
\text { Year }\end{array}$ & $\begin{array}{l}\text { End } \\
\text { Year }\end{array}$ & $\begin{array}{l}\text { Samp } \\
\text { les? }\end{array}$ & $\begin{array}{c}\text { Minimum } \\
\text { Value/\#Samp }\end{array}$ & $\begin{array}{r}\text { Maximum } \\
\text { value/STD }\end{array}$ & $\begin{array}{l}\text { Basis for } \\
\text { Uncertainty }\end{array}$ \\
\hline Co-60 & Unknown. & Unknown. & $T 12.970000000000$ & Cl & 1985 & 1985 & N & & & See continuation page. \\
\hline $\mathrm{Sr}-90$ & Unknown. & Unknown. & T. .01781000000000 & CI & 1985 & 1985 & N & & & See continuation page. \\
\hline Tc-99 & Unknown. & Unknown. & $T .00348500000000$ & CI & 1985 & 1985 & $N$ & & & See continuation page. \\
\hline $1-129$ & Unknown. & Unknown. & T. .00000090630000 & CI & 1985 & 1985 & N & & & See continuation page. \\
\hline Cs -137 & Unknown. & Unknown. & T 3.8720000000000 & CI & 1985 & 1985 & N & & & See continuation page. \\
\hline $\mathrm{Ce}-144$ & Unknown. & Unknown. & $T .09099000000000$ & CI & 1985 & 1985 & N & & & See continuation page. \\
\hline Eu-154 & Unknown. & Unknown. & T. .00005614000000 & CI & 1985 & 1985 & $\mathbf{N}$ & & & See continuation page. \\
\hline Eu-155 & Unknown. & Unknown. & T. .18200000000000 & CI & 1985 & 1985 & $\mathbf{N}$ & & & See continuation page. \\
\hline$U-234$ & Unknown. & Unknown. & T. .00004065000000 & CI & 1985 & 1985 & $\mathbf{N}$ & & & See continuation page. \\
\hline
\end{tabular}

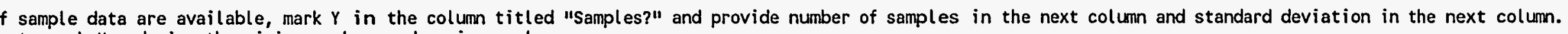
not, mark $N$ and give the minimum value and maximum value.

itional information or explanations (indicate pertinent contaminant)

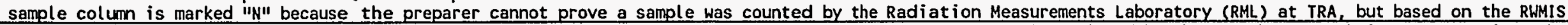

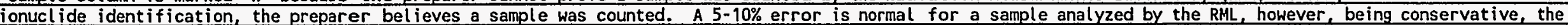
parer assigned a $20 \%$ error (doubling the $10 \%$ error). 


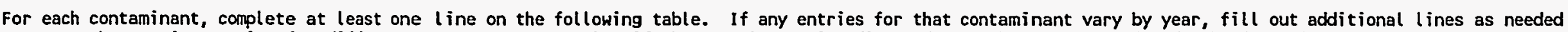

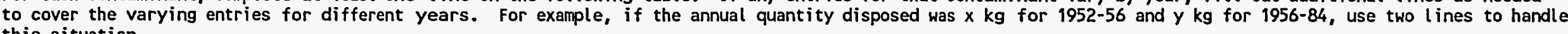
this situation.

\begin{tabular}{|c|c|c|c|c|c|c|c|c|c|c|}
\hline Radionucl ide & Physical Form & Chemical Form & $\begin{array}{l}\text { (A) nnual/(T)otal } \\
\text { Quantity }\end{array}$ & Unit & $\begin{array}{l}\text { Begin } \\
\text { Year }\end{array}$ & $\begin{array}{l}\text { End } \\
\text { Year }\end{array}$ & $\begin{array}{l}\text { Samp } \\
\text { les? }\end{array}$ & $\begin{array}{c}\text { Minimum } \\
\text { Value/\#Samp }\end{array}$ & $\begin{array}{r}\text { Maximum } \\
\text { Value/STD }\end{array}$ & $\begin{array}{l}\text { Basis for } \\
\text { Uncertainty }\end{array}$ \\
\hline $\mathrm{U}-235$ & Unknown. & Unknown. & T.00000087120000 & $\mathrm{Cl}$ & 1985 & 1985 & N & & & See continuation page. \\
\hline$u-236$ & Unknown. & Unknown. & T. .00001549000000 & $\mathrm{Cl}$ & 1985 & 1985 & N & & & See continuation page. \\
\hline Np-237 & Unknown. & Unknown. & $T .00002517000000$ & CI & 1985 & 1985 & N & & & See continuation page. \\
\hline Pu-238 & Unknown. & Unknown. & $T .00104500000000$ & CI & 1985 & 1985 & $\mathbf{N}$ & & & See continuation page. \\
\hline Pu-239 & Unknown. & Unknown. & T. .00104500000000 & CI & 1985 & 1985 & N & & & See continuation page. \\
\hline $\mathrm{Pu}-240$ & Unknown. & Unknown. & T. .00011030000000 & CI & 1985 & 1985 & N & & & See cont inuation page. \\
\hline Pu-241 & Unknown. & Unknown. & $\mathrm{T} .11420000000000$ & CI & 1985 & 1985 & N & & & See continuation page. \\
\hline Am-241 & Unknown. & Unknown. & T. .00052270000000 & CI & 1985 & 1985 & N & & & See continuation page. \\
\hline $\mathrm{Cm}-242$ & Unknown. & Unknown. & T. .00052270000000 & Cl & 1985 & 1985 & N & & & See cont inuation page. \\
\hline
\end{tabular}

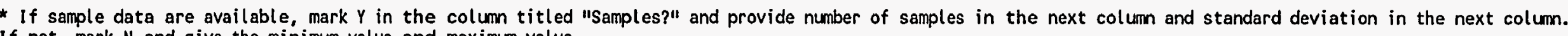
If not, mark $N$ and give the minimum value and maximum value.

Additional information or explanations (indicate pertinent contaminant)

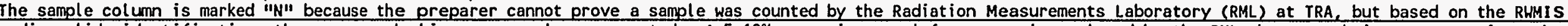

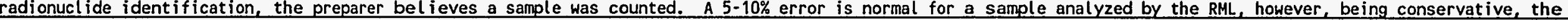
preparer assigned a $20 \%$ error (doubling the $10 \%$ error). 
- each contaminant, complete at least one line on the following table. If any entries for that contaminant vary by year, fill out additional lines as needed

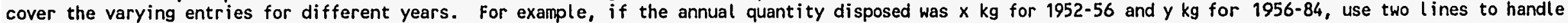
is situation.

\begin{tabular}{|c|c|c|c|c|c|c|c|c|c|c|}
\hline Radionuclide & Physical Form & Chemical Form & $\begin{array}{l}\text { (A) nnual/(T)otal } \\
\text { Quantity }\end{array}$ & Unit & $\begin{array}{l}\text { Beg in } \\
\text { Year }\end{array}$ & $\begin{array}{l}\text { End } \\
\text { Year }\end{array}$ & $\begin{array}{l}\text { Samp } \\
\text { les? }\end{array}$ & $\begin{array}{c}\text { Minimum } \\
\text { Value/\#Samp }\end{array}$ & $\begin{array}{r}\text { Maximum } \\
\text { Value/STD }\end{array}$ & $\begin{array}{l}\text { Basis for } \\
\text { Uncertainty }\end{array}$ \\
\hline $\mathrm{Cm}-244$ & Unknown. & Unknown. & $T .00048400000000$ & $\mathrm{Cl}$ & 1985 & 1985 & N & & & See cont inuation page. \\
\hline $\mathrm{Ce}-144$ & Unknown. & Unknown. & T 15.49000000000 & CI & 1985 & 1985 & N & $-20 \%$ & $+20 \%$ & See comment below. \\
\hline Co-60 & Unknown. & Unknown. & $T 143.64000000000$ & $\mathrm{CI}$ & 1985 & 1985 & N & $-20 \%$ & $+20 \%$ & See comment below. \\
\hline Cs -134 & Unknown. & Unknown. & T 24.390000000000 & $\mathrm{CI}$ & 1985 & 1985 & N & $-20 \%$ & $+20 \%$ & See comment below. \\
\hline Cs -137 & Unknown. & Unknown. & T 88.290000000000 & CI & 1985 & 1985 & N & $-20 \%$ & $+20 \%$ & See comment below. \\
\hline $\mathrm{Nb}-95$ & Unknown. & Unknown. & T 11.570000000000 & CI & 1985 & 1985 & N & $-20 \%$ & $+20 \%$ & See comment below. \\
\hline$U-238$ & Unknown. & Unknown. & T. .00120000000000 & CI & 1985 & 1985 & N & $-20 \%$ & $+20 \%$ & See comment below. \\
\hline $2 r-95$ & Unknown. & Unknown. & T 28.26000000000 & $\mathrm{CI}$ & 1985 & 1985 & N & $-20 \%$ & $+20 \%$ & See comment below. \\
\hline$H-3$ & Unknown. & Unknown. & T.72570000000000 & CI & 1986 & 1986 & N & & & See continuation page. \\
\hline
\end{tabular}

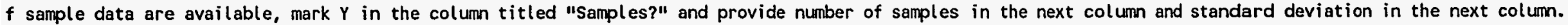
not, mark $N$ and give the minimum value and maximum value.

litional information or explanations (indicate pertinent contaminant)

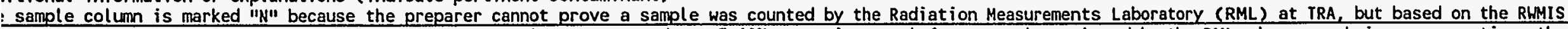

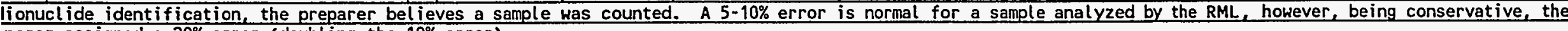
parer assigned a $20 \%$ error (doubling the 10\% error). 
each contaminant, complete at least one line on the following table. If any entries for that contaminant vary by year, fill out additional lines as needed

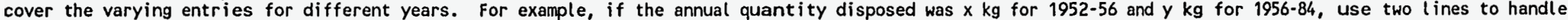
s situation.

\begin{tabular}{|c|c|c|c|c|c|c|c|c|c|c|}
\hline Radionuclide & Physical Form & Chemical form & $\begin{array}{l}\text { (A)nnual/(T)otal } \\
\text { Quant ity }\end{array}$ & Unit & $\begin{array}{l}\text { Begin } \\
\text { Year }\end{array}$ & $\begin{array}{l}\text { End } \\
\text { Year }\end{array}$ & $\begin{array}{l}\text { Samp } \\
\text { les? }\end{array}$ & $\begin{array}{c}\text { Minimum } \\
\text { Value/\#Samp }\end{array}$ & $\begin{array}{l}\text { Maximum } \\
\text { Value/STD }\end{array}$ & $\begin{array}{l}\text { Basis for } \\
\text { Uncertainty }\end{array}$ \\
\hline Ce- 144 & Unknown. & Unknown. & T. .04160000000000 & CI & 1986 & 1986 & N & & & See continuation page. \\
\hline Eu-154 & Unknown. & Unknown. & T. .00002566000000 & CI & 1986 & 1986 & N & & & See cont inuation page. \\
\hline$U-234$ & Unknown. & Unknown. & T. .00001858000000 & CI & 1986 & 1986 & $N$ & & & See cont inuation page. \\
\hline$U-235$ & Unknown. & Unknown. & T. .00000039820000 & $\mathrm{CI}$ & 1986 & 1986 & N & & & See continuation page. \\
\hline$U-236$ & Unknown. & Unknown. & T. .00000708000000 & CI & 1986 & 1986 & N & & & See continuation page. \\
\hline Np-237 & Unknown. & Unknown. & $T .00001150000000$ & CI & 1986 & 1986 & N & & & See continuation page. \\
\hline Pu-238 & Unknown. & Unknown. & $T .00047790000000$ & CI & 1986 & 1986 & N & & & See continuation page. \\
\hline Pu-239 & Unknown. & Unknown. & T. .00047790000000 & $\mathrm{Cl}$ & 1986 & 1986 & N & & & See continuation page. \\
\hline
\end{tabular}

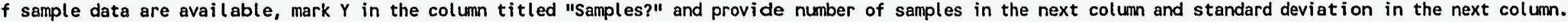
not, mark $\mathrm{N}$ and give the minimum value and maximum value.

itional information or explanations (indicate pertinent contaminant)

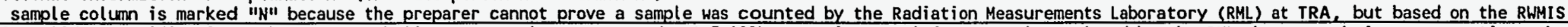

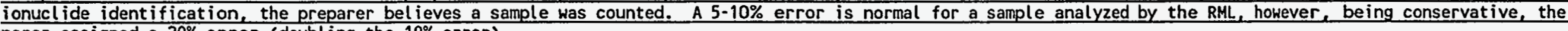
parer assigned a $20 \%$ error (doubl ing the 10\% error). 
- each contaminant, complete at least one line on the following table. If any entries for that contaminant vary by year, fill out additional lines as needed

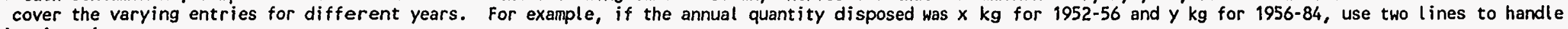
is situation.

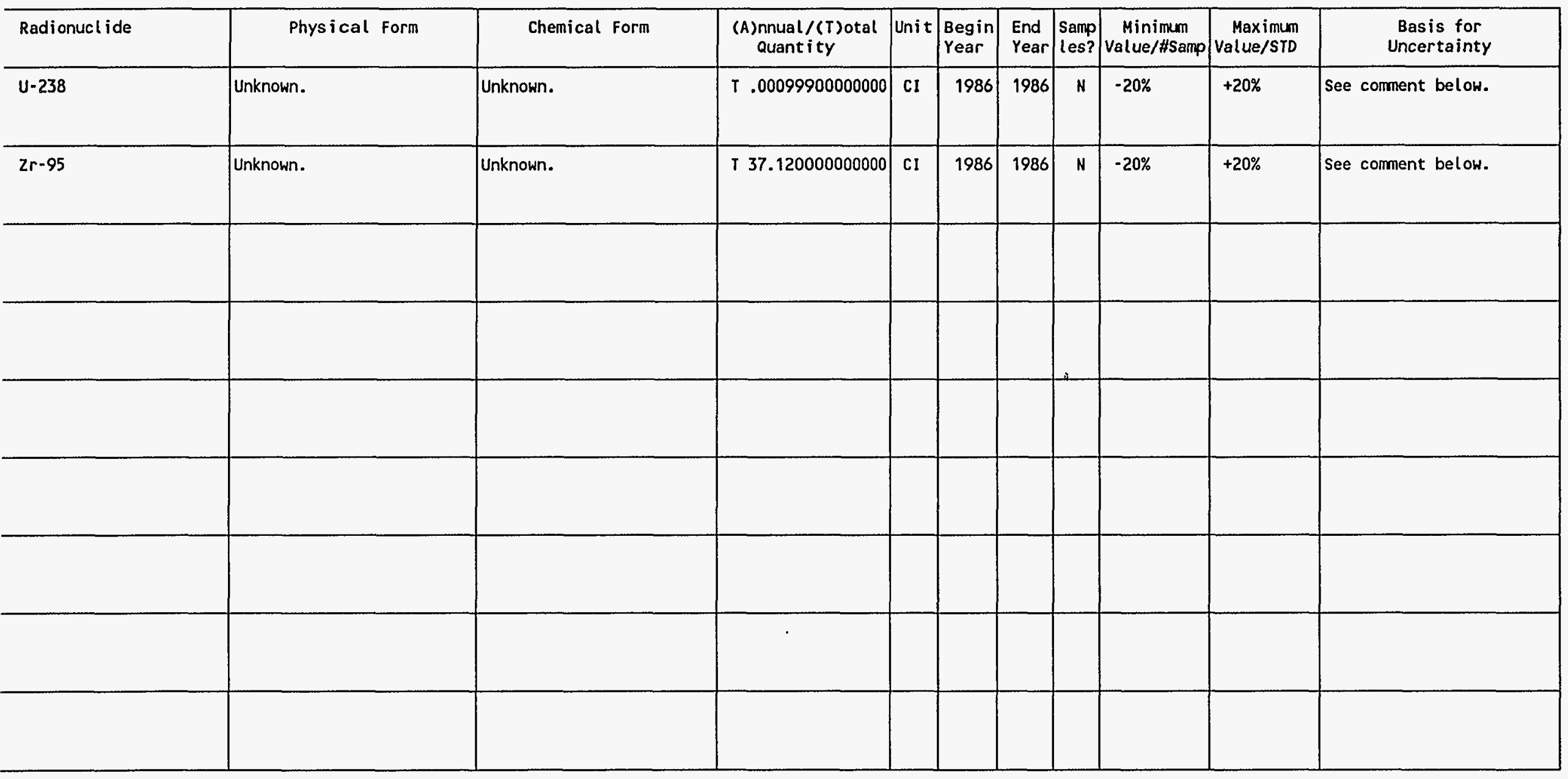

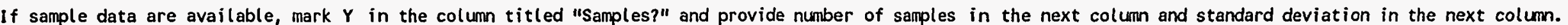
not, mark $N$ and give the minimum value and maximum value.

ditional information or explanations (indicate pertinent contaminant)

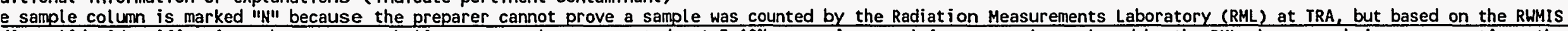

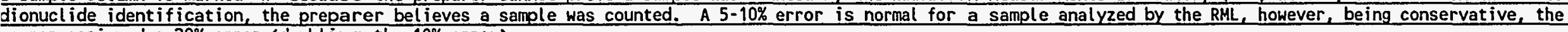
eparer assigned a $20 \%$ error (doubling the $10 \%$ error). 
- Type of source of information: (check box)

X] RWMIS [ ] other database

] sample analysis data

$x]$ operating records $[\mathrm{X}]$ interview

] expert judgment [ ] reports

] other

3. Do the estimates of contaminant fuantities in Part $C$ and $D$ represent:

X] best estimate

] worst case

] other

5. Do the data conflict with RWMIS? (Historical or Present Data Only) ] no

$x]$ yes

' Major unknowns in inventories of :ontaminants:

Inidentified beta gamma $(U-B-G)$ were inknown.
2. Details concerning source (names, report no., dates, etc.) Hot cell operation records along with interviewing a previous hot cell employee.

4. If other than best estimate, explain why:

6. If yes, explain why:

A portion of or all of the measurements were assumed to be taken using a G-M detector which only measures gamma emitters; beta emitters have been added to the RWMIS activity based on scaling factors. Therefore, the total activity will be greater than that in RWMIS.

8. Key assumptions used to deal with the unknowns: U-B-G radionuclides were chosen based on EPRI NP-5077 for dry active waste for PWRs. PWR was chosen because it is similar to ATR, the reactor at TRA. The radionuclides were chosen based on a combination of the following: cross section, half-lives, and their fission product. 
ontinuation of Part $\underline{D}$

Column or Question Number or Title Uncertainty basis.

ecause of the form (the various types and shapes) of the material, it is not known whether a ipment's activity was determined with a G-M counter or with a NAI detector, using smears. erefore, the true activity and uncertainty is unknown。 The G-M counter method has been assumed as method used to estimate the gamma emitting radionuclides. Although a G-M counter cannot timate alpha emitting radionuclides, the alpha activities were scaled from the gamma activities. ierefore, the G-M counter correction applies for the alpha emitters, CIDRA calculated best timate, and upper and lower bounds, accordingly. 
DATA INPUT FOR HISTORICAL DATA TASK FOR RWMC SUBSURFACE DISPOSAL AREA

PART A - GENERAL INFORMATION RDT - 279

1. Preparer: Amaro, C.

3. Generator: TRA

(area or contractor - use code from attached list)

5. Number of waste stream from this facility: $3 \mathrm{R}$

7. Type of radioactive waste (check box):

[ ] TRU or suspect TRU

[X] LLW

[ ] non-radioactive

8. Actual years disposed of at.SDA: starting year 1991 Ending year 1993
2. Date prepared: 07/03/94

4. Particular facility: 632

(building number - use code from attached list)

6. Waste stream: Absorbed liquids.

9. Waste stream volume:
Amount
Check box: [ ] 1.1392 units cubic meters.
Check box: [ ] container. volume or [X] waste volume

10. Comments (specify number of pertinent question):

4. Also TRA-ERP, which the preparer believes is building 603 . 
- General physical form (see attached list) 2. Details on physical form(particularly confinement related) X] other (specify) ther liquid setups. Liquid has been absorbed by another material.

Chemical form: nknown.

- Waste container type (see attached list) poden box.

Comments (specify number of pertinent question):

5. Also RDL.
4. Inner packaging: [ ] plastic bag [X] plastic liner [ ] metal liner [ ] none [ ] other (specify)

\section{Other characteristics of interest:}




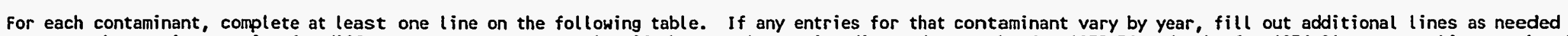

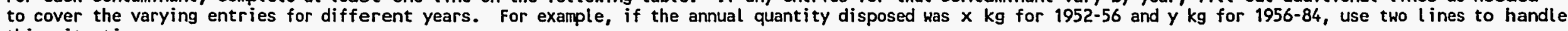
this situation.

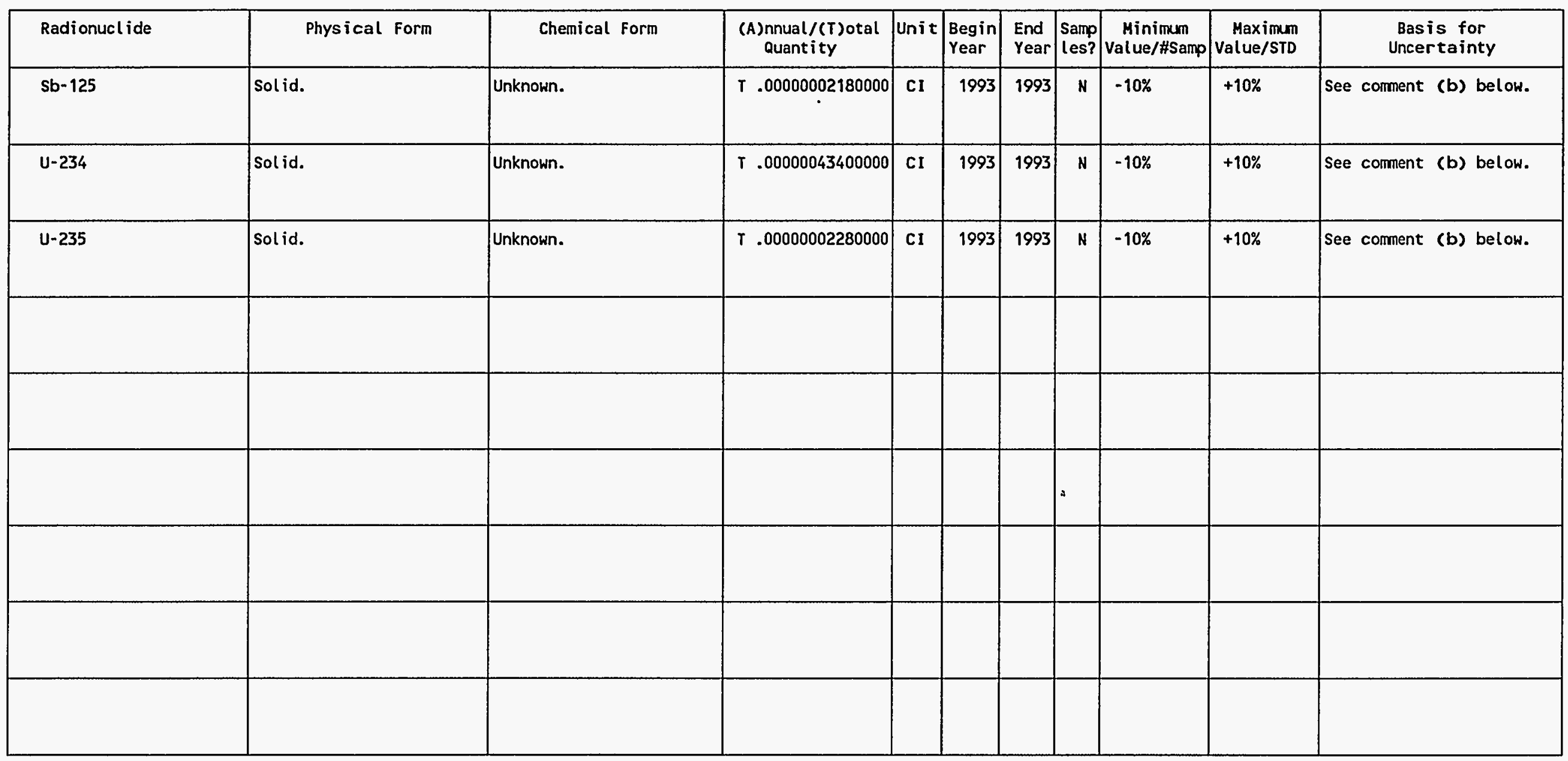

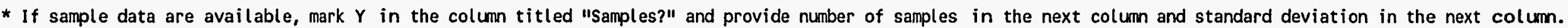
If not, mark $N$ and give the minimum value and maximum value.

Additional information or explanations (indicate pertinent contaminant)

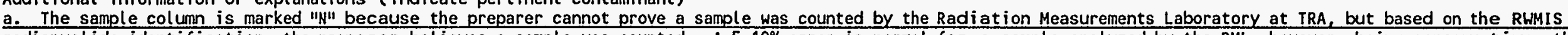

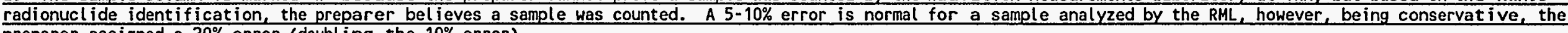
preparer assigned a $20 \%$ error (doubling the 10\% error).

b. A sample(s) was analyzed by the RML. Based on professional judgment, the total uncertainty for the sample(s) was calculated to be approximately $+/-10 \%$. 
- Type of source of information: check box)

X] RWMIS [ ] other database

X] sample analysis data

] operating records $[\mathrm{X}]$ interview

expert judgment $[X]$ reports

] other

- Do the estimates of contaminant

uantities in Part $C$ and $D$ represent:

$x]$ best estimate

] worst case

] other

- Do the data conflict with RWMIS?

(Historical or Present Data only)

$X]$ no

] yes

- Major unknowns in inventories of ontaminants :

AP and MFP were unknown.
2. Details concerning source (names, report no., dates, etc.) The preparer of this report shipped some of this material. It is characterized in WROC-WCR-007-93 Write up. C.R. Amaro, "Amalgamation of Low-level Waste".

4. If other than best estimate, explain why:

6. If yes, explain why:

8. Key assumptions used to deal with the unknowns: MAP and MFP radionuclides were chosen based on EPRI NP-5077 for dry active waste for PWRs. PWR was chosen because it is similar to ATR, the reactor at TRA. The radionuclides were chosen based on a combination of the following: cross section, half-lives, and their fission products. 
DATA INPUT FOR HISTORICAL DATA TASK FOR RWMC SUBSURFACE DISPOSAL AREA

PART A - GENERAL INFORMATION RDT - 280

1. Preparer: Amaro, C.

3. Generator: TRA

(area or contractor - use code from attached list)

5. Number of waste stream from this facility: 7R

7. Type of radioactive waste (check box):

[ ] TRU or suspect TRU

[X] LLW

[ ] non-radioactive

8. Actual years disposed of at SDA: Starting year 1987 Ending year 1992
2. Date prepared: $07 / 03 / 94$

4. Particular facility: 642

(building number - use code from attached list)

6. Waste stream:

Combustible material, such as paper, wood, plastic, rags and wipes.

9. Waste stream volume:

Amount

89.2421 Units Cubic meters.

Check box: [ ] annual or [X] total over all years

Check box: [ ] container volume or [X] waste volume

10. Comments (specify number of pertinent question):

4. Waste is actualiy from $603,632,670$, and TRA-B\&W; the preparer could not determine what facility TRA-B\&W was. Stream is same type of content as TRA-642-7 from earlier period. 


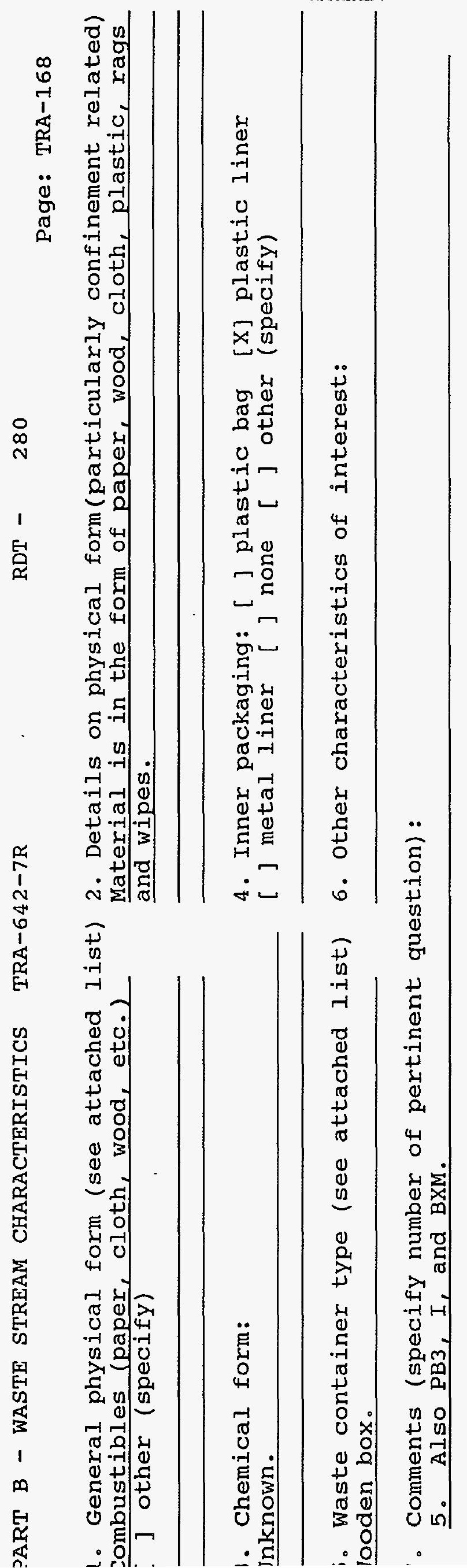




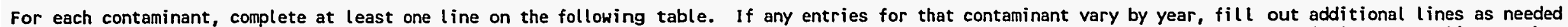

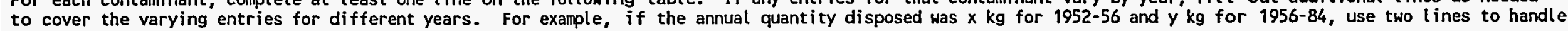
this situation.

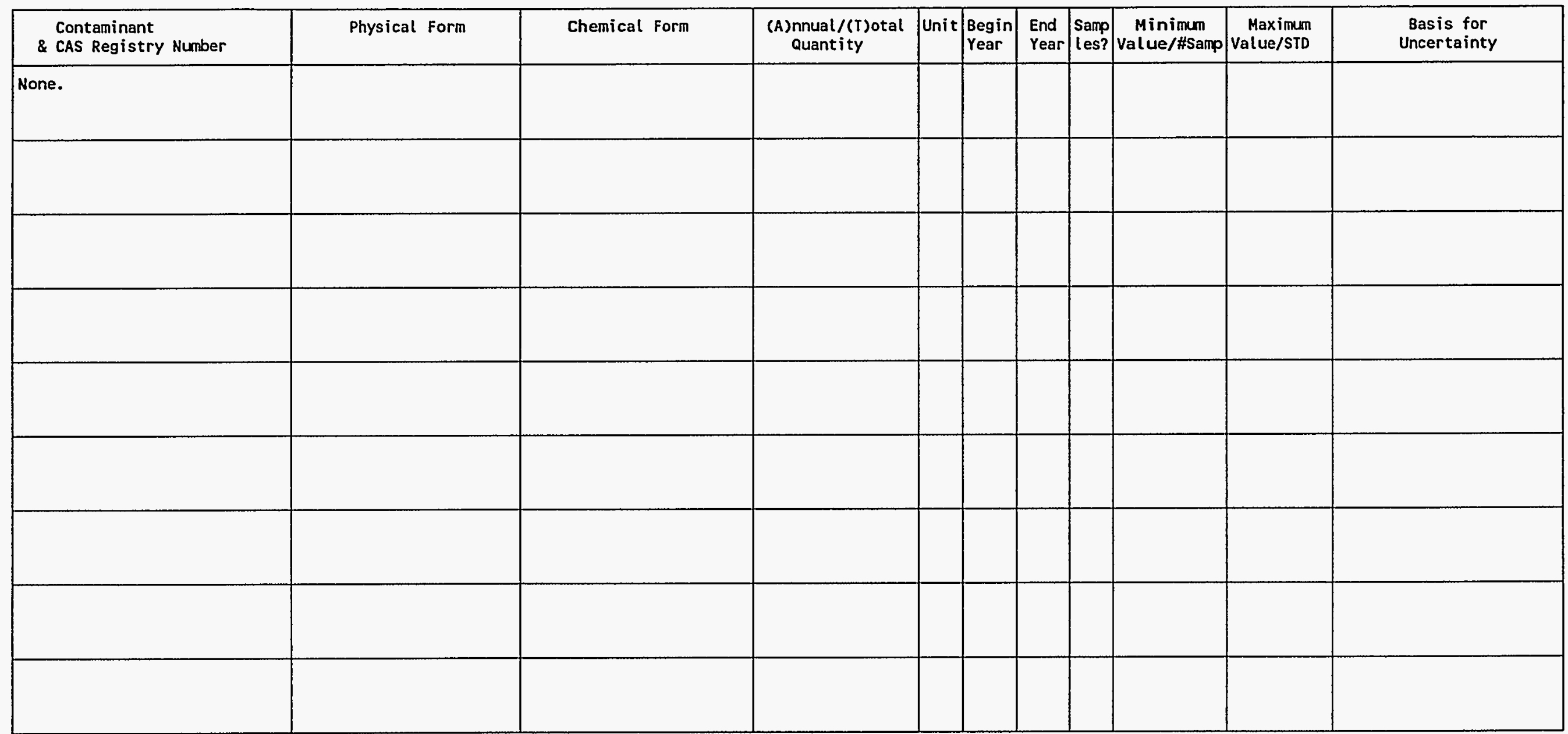

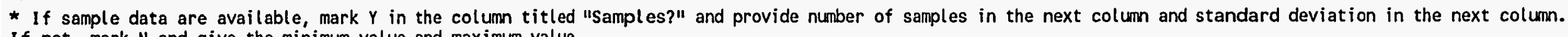
If not, mark $N$ and give the minimum value and maximum value.

Additional information or explanations (indicate pertinent contaminant) 


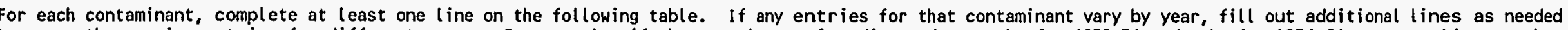

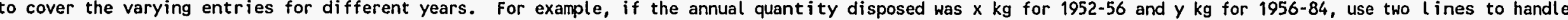
this situation.

\begin{tabular}{|c|c|c|c|c|c|c|c|c|c|c|}
\hline Radionucl ide & Physical Form & Chemical Form & $\begin{array}{l}\text { (A) nnual / ( T )otal } \\
\text { Quantity }\end{array}$ & Unit & $\begin{array}{l}\text { Begin } \\
\text { Year }\end{array}$ & $\begin{array}{l}\text { End } \\
\text { Year }\end{array}$ & $\begin{array}{l}\text { Samp } \\
\text { les? }\end{array}$ & $\begin{array}{c}\text { Minimum } \\
\text { Value/\#Samp }\end{array}$ & $\begin{array}{r}\text { Maximum } \\
\text { Value/STD }\end{array}$ & $\begin{array}{l}\text { Basis for } \\
\text { Uncertainty }\end{array}$ \\
\hline$H-3$ & Particulate. & Unknown. & $T .05741000000000$ & $\mathrm{Cl}$ & 1987 & 1987 & N & & & See continuation page. \\
\hline C- 14 & Particulate. & Unknown. & T .00770100000000 & CI & 1987 & 1987 & N & & & See continuation page. \\
\hline Fe-55 & Particulate. & Unknown. & T 1.330000000000 & Cl & 1987 & 1987 & N & & & See continuation page. \\
\hline $\mathrm{Ni}-59$ & Particulate. & Unknown. & $r .00039900000000$ & $\mathrm{CI}$ & 1987 & 1987 & N & & & See continuation page. \\
\hline $\mathrm{Ni}-63$ & Particulate. & Unknown. & T. .2240000000000 & $\mathrm{CI}$ & 1987 & 1987 & N & & & See continuation page. \\
\hline Co-60 & Particulate. & Unknown. & T. .46900000000000 & CI & 1987 & 1987 & N & & & See continuation page. \\
\hline $\mathrm{Sr}-90$ & Particulate. & Unknown. & $T .0006441000000$ & CI & 1987 & 1987 & N & & & See continuation page. \\
\hline Tc-99 & Particulate. & Unknown. & $T .00012600000000$ & $\mathrm{CI}$ & 1987 & 1987 & N & & & See continuation page. \\
\hline $1-129$ & Particulate. & Unknown. & T. .00000003276000 & CI & 1987 & 1987 & N & & & See continuation page. \\
\hline
\end{tabular}

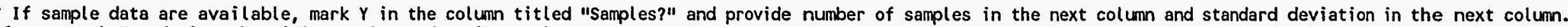
$f$ not, mark $N$ and give the minimum value and maximum value.

dditional information or explanations (indicate pertinent contaminant)

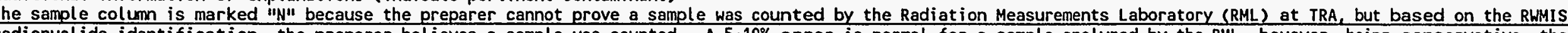

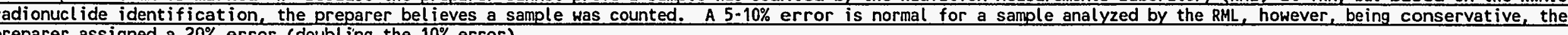
reparer assigned a $20 \%$ error (doubl ing the $10 \%$ error). 
- Type of source of information: check box)

X] RWMIS [ ] other database

] sample analysis data

] operating records [ ] interview

] expert judgment [ ] reports

] other

Do the estimates of contaminant uantities in Part $C$ and $D$ represent: X] best estimate

] worst case

] other

- Do the data conflict with RWMIS? (Historical or Present Data Only) ] no

$x]$ yes

- Major unknowns in inventories of ontaminants :

$A P$ and MFP and $U-B-G$ (unidentified eta-gamma) were unknown.
2. Details concerning source (names, report no., dates, etc.)

4. If other than best estimate, explain why:

6. If yes, explain why:

A portion of or all of the measurements were assumed to be taken using a G-M detector which only measures gamma emitters; beta emitters have been added to the RWMIS activity based on scaling factors. Therefore, the total activity will be greater than that in RWMIS.

8. Key assumptions used to deal with the unknowns:

MAP, MFP and U-B-G, were chosen based on EPRI NP-5077 for dry active waste for PWRs. PWR was chosen because it is similar to ATR, the reactor at TRA. The radionuclides were chosen based on a combination of the following: cross section, half-lives, and their fission products. 
Continuation of Part $\underline{D}$

Column or Question Number or Title Uncertainty basis.

Because of the various types of materials, it is not known whether a shipment's activity was determined with a G-M counter or a NAI detector using smears. Therefore, the true activity and uncertainty is unknown. The G-M counter method has been assumed as the method used to estimate the gamma emitting radionuclides. Although a G-M counter cannot estimate alpha emitting radionuclides, the alpha activities were scaled from the gamma activities. Therefore, the G-M counter correction applies for the alpha emitters, CIDRA calculated best estimate, and upper and lower bounds, accordingly. 
DATA INPUT FOR HISTORICAL DATA TASK FOR RWMC SUBSURFACE DISPOSAL AREA

ART A - GENERAL INFORMATION RDT - 299

- Preparer: Amaro, C.

- Generator: TRA

area or contractor - use code from attached list)

Number of waste stream from this facility:

IR

- Type of radioactive waste (check box):

] TRU or suspect TRU

X] LLW

] non-radioactive

Actual years disposed of at SDA:

tarting year 1993 Ending year 1993
2. Date prepared: 07/03/94

4. Particular facility: 670

(building number - use code from attached list)

6. Waste stream:

Beryllium reflectors from ATR.

9. Waste stream volume:

Amount 2.2920 Units Cubic meters.

Check box: [ ] annual or [X] total over all years

Check box: [ ] container volume or [X] waste volume

0. Comments (specify number of pertinent question): 


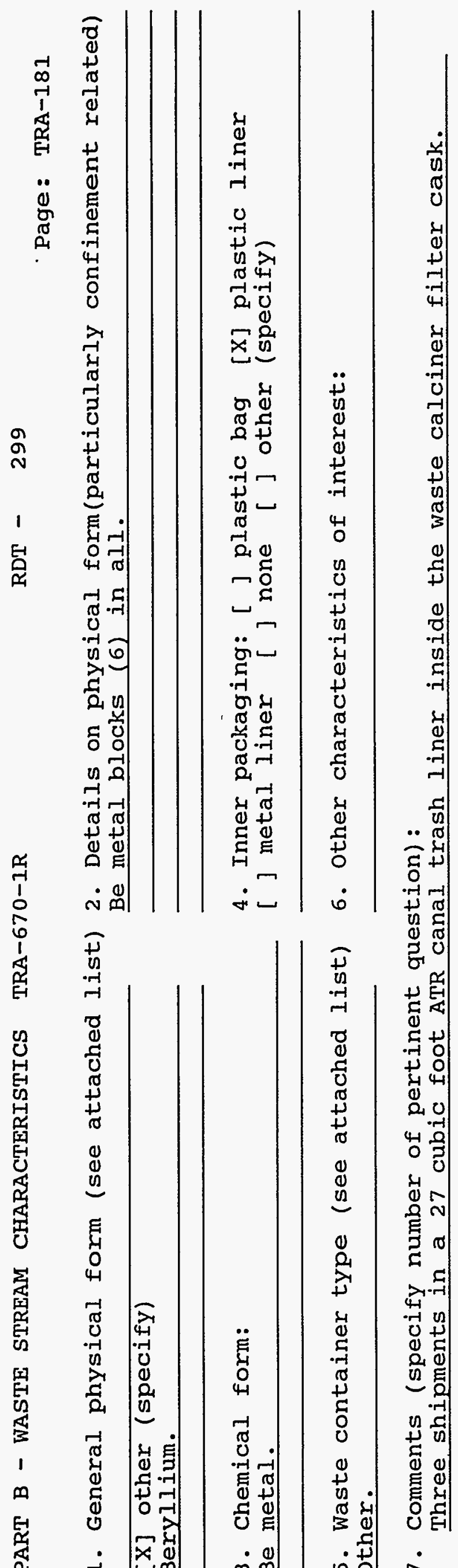


Type of source of information: neck box)

RWMIS [ ] other database sample analysis data operating records [X] interview expert judgment [ ] reports other

Do the estimates of contaminant antities in Part $C$ and $D$ represent: best estimate worst case other

Do the data conflict with RWMIS? (Historical or Present Data only) no. yes

Major unknowns in inventories of ataminants:

ne.
2. Details concerning source (names, report no., dates, etc.) See Continuation Page of Part E.

4. If other than best estimate, explain why:

6. If yes, explain why:

8. Key assumptions used to deal with the unknowns:

This waste stream was removed from the reactor about 1986. Short-lived radionuclides, such as $\mathrm{Cr}-51$ and $\mathrm{Fe}-59$, have decayed by 1993 to extremely small activities and are not included here. 
ontinuation of Part $\underline{E}$

Column or Question Number or Title 2.

.A. Jacobi letter to T.L. Clements, JAJ-006-93, "Beryllium Reflector Component Disposal Response," ept.17, 1993; J.0. Bower letter to R.N. Bhatt, JoB-09-94, "ATR Beryllium Inventory", March 16, 994; S.J. Maheras, et al, "Radioactive Waste Management Comples Low-Level Waste Radiological Waste adiological Performance Assessment", EGG-WM-8773, I.N.E.L., May 1994; P.K. Nagata distribution, Letter Report on Tritium Release from Buried Beryllium Reflectors", Dec. 14, 1994; T.A. Tomberlin etter to P.K.Nagata, TOM-1-94, "Review of Letter Report on Tritium Release from Buried Beryllium eflectors", Jan. 19, 1994; T.A. Tomberlin letter to M.M. Garland, ToM-2-94, "Projected Tritium nventory in the ATR Reflector at the End of Cycle 102B-1", Jan. 26, 1994; T. A. Tomberlin letter to .J. Toomer, TOM-7-92, "Characterization Update for Beryllium Reflector Components in the ATR anal", Nov. 5, 1992; Communication from T. Hopkins, EG\&G Idaho, Dec. 22, 1992; Communication from .J. Maheras to R.N. Beatty, EG\&E Idaho, Aug. 31, 1992; W.W. Gary letter to J.A. Lake, WWG-362-92, Disposition of ATR Beryllium"; G.R. Longhurst letter to J. G. Crocker, GRL-15-91, "Disposition of TR Beryllium", May 6, 1991; G.R. Longhurst letter to W.W. Gay, GRL-13-91, "Experiment Core and omponents Disposition", April 16, 1991; D. R. Wilkinson letter to L.J. Toomer, DWR-77-92, "Final eview and Evaluation for Test Reactors Area (TRA) Advanced Test Reactor (ATR) Waste haracterization Report", Oct. 20, 1992; T. A. Tomberlin letter to R.N. Beatty, TOM-04-91, Characterization of ATR Beryllium Reflector Components in the ATR Canal as of April 15, $1991 "$ pril 19, 1991; T.A. Tomberlin letter to R.N. Beatty, TOM-05-91, "Characterization of Outer Shim control cylinders in the ATR Canal as of April 15, 1991", May 8, 1991; J.A. Jacobi letter to D.R. ilkinson, JAJ-30-92, "Characterization of Beryllium Waste Stream", July 10, 1992; J.D. Wells letter o L.J. Toomer, JDW-43-92, "Waste Package Containment Requirements", Aug. 11, 1992; R.N. Beatty etter to S.J. Maheras, RNB-11-92, "Estimated Isotope Activity in ATR Beryllium Reflector Blocks and uter Shim Control Cylinders", June 11, 1992; D.R. Wilkinson letter to D.J. Bright, DRW-39-92, "Test eactors Area Advanced Test Reactor (TRA/ATR) Beryllium Action Item DLF \#324 Response", May 15, 992; F.C. Fogarty letter to H.P. Mann, FCF-146-92, "Disposal of Expended Beryllium Reflector laterial", May 11, 1992; G.R. Longhurst distribution, GRL-20-92, "Reclamation of ATR Beryllium", larch 23, 1992; R.M. Brown letter to A.D. Rodgers, RMB-01-88, "ATR Beryllium/Tritium Waste", OCt. 7, 987; B.R. Baldwin letter to R.A. Brown, BRB-17-87, "Tritium Data for ATR Beryllium Blocks", May 8, 987; B.R. Baldwin letter to D.W. Suthers, BRB-10-87, "Report of RWMC Beryllium Meeting", May 8, 987; T.A. Tomberlin letter to D.E. Sheldon, TOM-30-86, "Tritium Content and stability in Beryllium tored in ATR Canal", Dec. 22, 1986; J.O. Brower letter to R.N. Bhatt, JoB-23-94, "ATR Tritium Waste nventory", Oct. $28,1994$. 
DATA INPUT FOR HISTORICAL DATA TASK FOR RWMC SUBSURFACE DISPOSAL AREA

RT A - GENERAL INFORMATION PDT - 304

Preparer: Amaro, $C$.

Generator: TRA

rea or contractor - use code from attached list)

Number of waste stream from this facility: $1 \mathrm{P}$

Type of radioactive waste (check box):

] TRU or suspect TRU

LLW

non-radioactive

Actual years disposed of at SDA: arting year 1994 Ending year 2003
2. Date prepared: $07 / 16 / 94$

4. Particular facility: 603

(building number - use code from attached list)

6. Waste stream:

Resin.

9. Waste stream volume:

Amount $\quad 425.4600$ Units Cubic meters.

Check box: [ ] annual or [X] total over all years

Check box: [ ] container volume or [X] waste volume

Comments (specify number of pertinent question):

4. Also 670 and 632 . 
General physical form (see attached list) 2. Details on physical form(particularly confinement related) in. Granular. other (specify)

\section{Chemical form:}

nown.

Waste container type (see attached list) er.

Comments (specify number of pertinent question): 5. Also probably RD5; 0 is a cask.
4. Inner packaging: [ ] plastic bag [ ] plastic liner [] metal liner [ ] none [X] other (specify) other.

6. Other characteristics of interest: 
each contaminant, complete at least one line on the following table. If any entries for that contaminant vary by year, fill out additional lines as needed

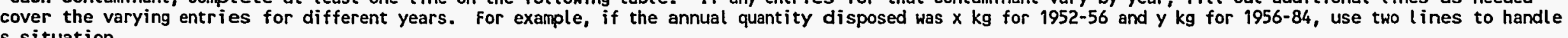
s situation.

\begin{tabular}{|c|c|c|c|c|c|c|c|c|c|c|c|}
\hline Radionuclide & Physical Form & Chemical Form & $\begin{array}{l}\text { (A)nnual } /(T) \text { otal } \\
\text { Quant ity }\end{array}$ & Unit & $\begin{array}{l}\text { Begin } \\
\text { Year }\end{array}$ & $\begin{array}{l}\text { End } \\
\text { Year }\end{array}$ & $\begin{array}{l}\text { Fore } \\
\text { cast }\end{array}$ & $\begin{array}{l}\text { Samp } \\
\text { Les? }\end{array}$ & $\begin{array}{c}\text { Minimum } \\
\text { Value/\#Samp }\end{array}$ & $\begin{array}{r}\text { Maximum } \\
\text { Value/STD }\end{array}$ & $\begin{array}{l}\text { Basis for } \\
\text { Uncertainty }\end{array}$ \\
\hline $\mathrm{Sr}-90$ & Resin. & Unknown. & T 2.7820000000000 & CI & 1994 & 2003 & $Y$ & N & & & \\
\hline $2 r-95$ & Resin. & Unknown. & T 1.1150000000000 & CI & 1994 & 2003 & $Y$ & N & & & \\
\hline $\mathrm{Nb}-95$ & Resin. & Unknown. & T 1.0920000000000 & $\mathrm{CI}$ & 1994 & 2003 & $Y$ & N & & & \\
\hline Mo-99 & Resin. & Unknown. & T. .19000000000000 & $\mathrm{CI}$ & 1994 & 2003 & $Y$ & N & & & \\
\hline$R u-103$ & Resin. & Unknown. & T. .12950000000000 & CI & 1994 & 2003 & $Y$ & N & & & \\
\hline$I-131$ & Resin. & Unknown. & T. .00200000000000 & CI & 1994 & 2003 & Y & N & & & \\
\hline$x e-133$ & Resin. & Unknown. & $\tau .00400000000000$ & CI & 1994 & 2003 & $Y$ & N & & & \\
\hline Cs- 134 & Resin. & Unknown. & T 1.0420000000000 & CI & 1994 & 2003 & $Y$ & N & & & \\
\hline Cs- 137 & Resin. & Unknown. & $T .40430000000000$ & CI & 1994 & 2003 & $Y$ & N & & & \\
\hline
\end{tabular}

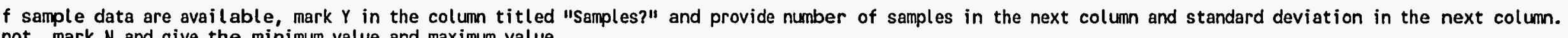
not, mark $N$ and give the minimum value and maximum value.

itional information or explanations (indicate pertinent contaminant) 
- Type of source of information: check box)

] RWMIS [ ] other database

] sample analysis data

operating records [ ] interview

] expert judgment [ ] reports

$\mathrm{x}$ ] generator forecasts

] other

- Do the estimates of contaminant uantities in Part $C$ and $D$ represent:

\section{$\mathrm{X}$ ] best estimate}

worst case

] other.

- Do the data conflict with RWMIS? (Historical or Present Data only) $\mathrm{X}]$ no

] Yes

- Major unknowns in inventories of ontaminants:

one.
2. Details concerning source (names, report no., dates, etc.) These numbers were based on S.J. Keating's letter to V.C. Randall, SJK-005-93, "Test Reactor Area (TRA) Radioactive Waste Forecasts", November 17, 1993.

4. If other than best estimate, explain why:

6. If yes, explain why:

8. Key assumptions used to deal with the unknowns: 


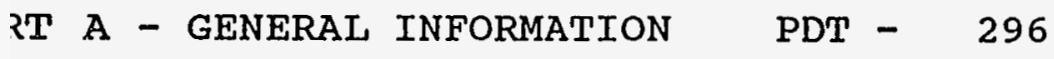

waste stream volume:

Amount 22.9200 Units Cubic meters.

Check box: [ ] annual or $[\mathrm{X}]$ total over all years

Check box: [ ] container volume or [X] waste volume

Comments (specify number of pertinent question): 4. This waste stream is the same as TRA-603-4, but is from 670 . 
General physical form (see attached list) ther core, reactor vessel, loop component ] other (specify)

- Chemical form:

etallic, such as stainless steel and luminum.

- Waste container type (see attached 1ist) thèr.

- Comments (specify number of pertinent question): 5. And $I ; O$ cannot be defined by the preparer.
2. Details on physical form (particularly confinement related) Solids, such as loop piping, stainless steel and aluminum pieces, equipment leads and pile tubes or end boxes from fuel elements.

4. Inner packaging: [ ] plastic bag [X] plastic liner [ ] metal liner [ ] none [ ] other (specify)

\section{Other characteristics of interest:}


each contaminant, complete at least one line on the following table. If any entries for that contaminant vary by year, fill out additional lines as needed

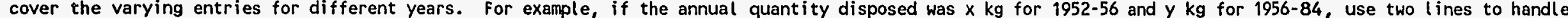
s situation.

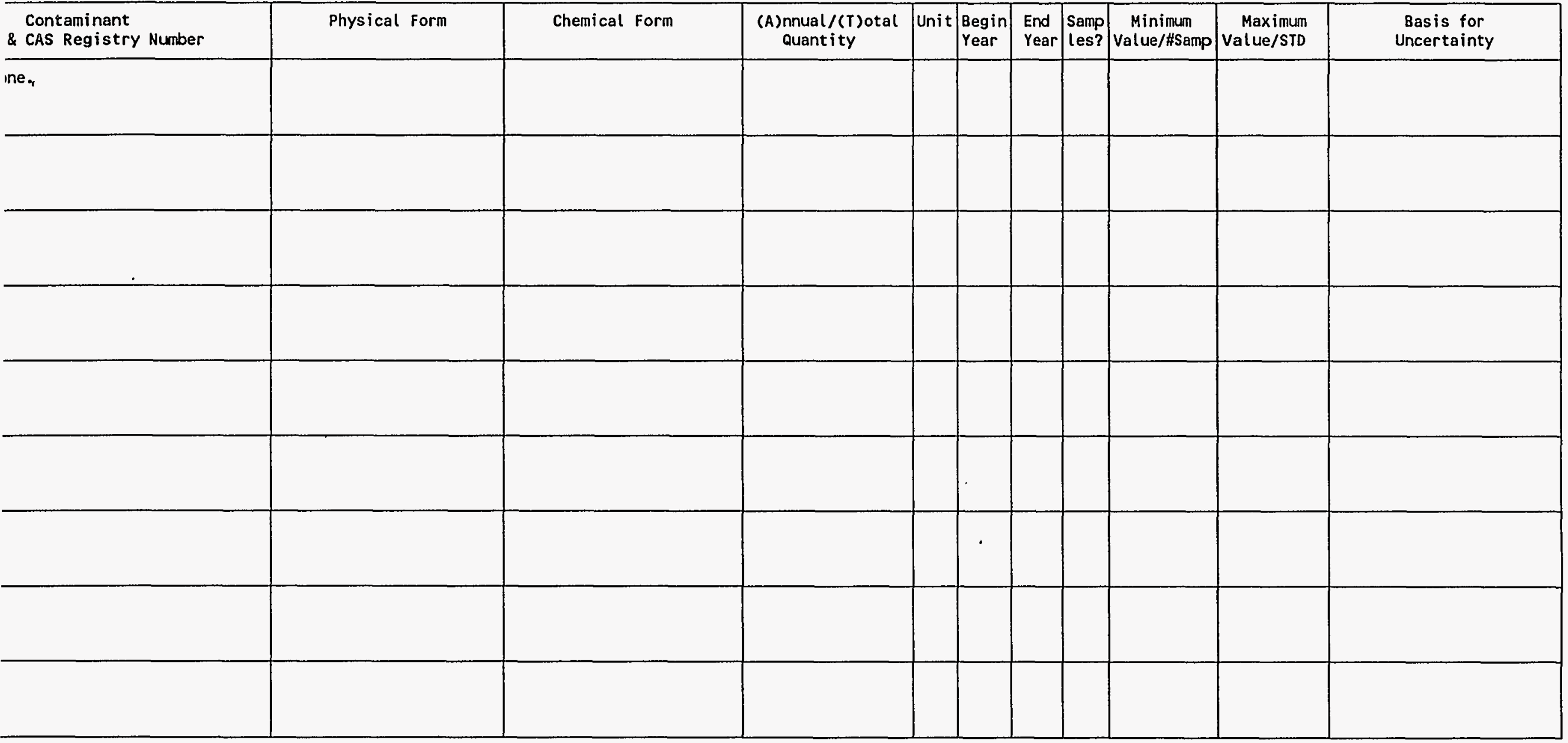

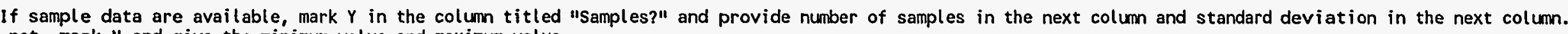
not, mark $N$ and give the minimum value and maximum value.

ditional information or explanations (indicate pertinent contaminant) 
each contaminant, complete at least one line on the following table. If any entries for that contaminant vary by year, fill out additional lines as needed over the varying entries for different years. For example, if the annual quantity disposed was $x \mathrm{~kg}$ for $1952-56$ and $y$ kg for $1956-84$, use two lines to handle situation.

\begin{tabular}{|c|c|c|c|c|c|c|c|c|c|c|c|}
\hline Radionuclide & Physical form & Chemical form & $\begin{array}{l}\text { (A)nnual/(T)otal } \\
\text { Quantity }\end{array}$ & Unit & $\begin{array}{l}\text { Begin } \\
\text { Year }\end{array}$ & $\begin{array}{l}\text { End } \\
\text { Year }\end{array}$ & 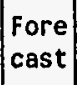 & $\begin{array}{l}\text { Samp } \\
\text { les? }\end{array}$ & $\begin{array}{c}\text { Minimum } \\
\text { Value/\#Samp }\end{array}$ & $\begin{array}{r}\text { Maximum } \\
\text { value/STD }\end{array}$ & $\begin{array}{l}\text { Basis for } \\
\text { Uncertainty }\end{array}$ \\
\hline Cs -137 & Metallic. & Unknown. & T 283.80000000000 & CI & 1994 & 1994 & N & N & & & \\
\hline $\mathrm{Ce}-144$ & Metallic. & Unknown. & T 6.6700000000000 & CI & 1994 & 1994 & N & N & & & \\
\hline Eu-154 & Metallic. & Unknown. & T. .00411600000000 & $\mathrm{CI}$ & 1994 & 1994 & N & N & & & \\
\hline$E u-155$ & Metallic. & Unknown. & T 13.340000000000 & CI & 1994 & 1994 & N & N & & & \\
\hline$U-234$ & Metallic. & Unknown. & T. .00298000000000 & $\mathrm{CI}$ & 1994 & 1994 & N & N & & & \\
\hline$U-235$ & Metallic. & Unknown. & T. .00006387000000 & $\mathrm{CI}$ & 1994 & 1994 & N & N & & & \\
\hline$U-236$ & Metallic. & Unknown. & T. .00113500000000 & $\mathrm{Cl}$ & 1994 & 1994 & N & $N$ & & & \\
\hline Np-237 & Metallic. & Unknown. & $T .00184500000000$ & $\mathrm{CI}$ & 1994 & 1994 & N & N & & & \\
\hline Pu-238 & Metallic. & Unknown. & T. .07664000000000 & CI & 1994 & 1994 & N & $N$ & & & \\
\hline
\end{tabular}

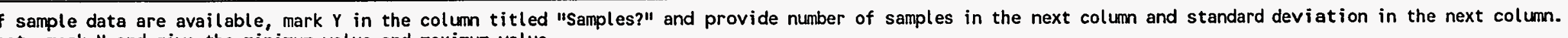
not, mark $N$ and give the minimum value and maximum value.

itional information or explanations (indicate pertinent contaminant) 
each contaminant, complete at least one line on the following table. If any entries for that contaminant vary by year, fill out additional lines as needed

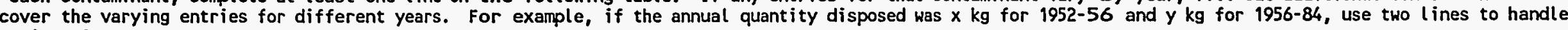
s situation.

\begin{tabular}{|c|c|c|c|c|c|c|c|c|c|c|c|}
\hline Radionucl ide & Physical Form & . Chemical Form & $\begin{array}{l}\text { (A)nnual/(T)otal } \\
\text { Quantity }\end{array}$ & Unit & $\begin{array}{l}\text { Begin } \\
\text { Year }\end{array}$ & $\begin{array}{l}\text { End } \\
\text { Year }\end{array}$ & $\begin{array}{l}\text { Fore } \\
\text { cast }\end{array}$ & $\begin{array}{l}\text { Samp } \\
\text { les? }\end{array}$ & $\begin{array}{c}\text { Minimum } \\
\text { Value/\#Samp }\end{array}$ & $\begin{array}{r}\text { Maximum } \\
\text { Value/STD }\end{array}$ & $\begin{array}{l}\text { Basis for } \\
\text { Uncertainty }\end{array}$ \\
\hline Pu-239 & Metallic. & Unknown. & T. .07664000000000 & $\mathrm{Cl}$ & 1994 & 1994 & $N$ & N & & & \\
\hline Pu-240 & Metallic. & Unknown. & $T .00809000000000$ & CI & 1994 & 1994 & $\mathbf{N}$ & N & & & \\
\hline Pu-241 & Metallic. & Unknown. & T 8.3730000000000 & CI & 1994 & 1994 & N & N & & & \\
\hline Am-241 & Metallic. & Unknown. & $T .03832000000000$ & CI & 1994 & 1994 & N & N & & & \\
\hline $\mathrm{Cm}-242$ & Metallic. & Unknown. & $T .03832000000000$ & CI & 1994 & 1994 & N & N & & & \\
\hline $\mathrm{Cm}-244$ & Metallic. & Unknown. & T. .03548000000000 & CI & 1994 & 1994 & N & N & & & \\
\hline$H-3$ & Metallic. & Unknown. & T 116.4000000000 & CI & 1995 & 1995 & $\mathbf{N}$ & N & & & \\
\hline C- 14 & Metallic. & Unknown. & T 15.610000000000 & CI & 1995 & 1995 & N & N & & & \\
\hline $\mathrm{Fe}-55$ & Metallic. & Unknown. & T 2697.0000000000 & CI & 1995 & 1995 & $\mathbf{N}$ & N & & & \\
\hline
\end{tabular}

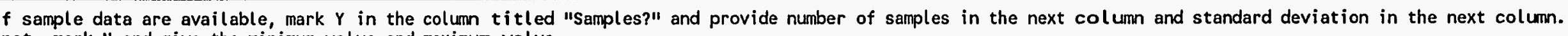
not, mark $N$ and give the minimum value and maximum value.

litional information or explanations (indicate pertinent contaminant) 


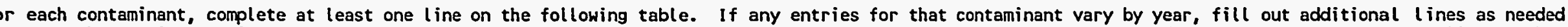

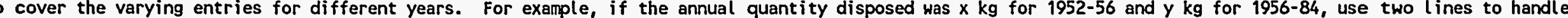
is situation.

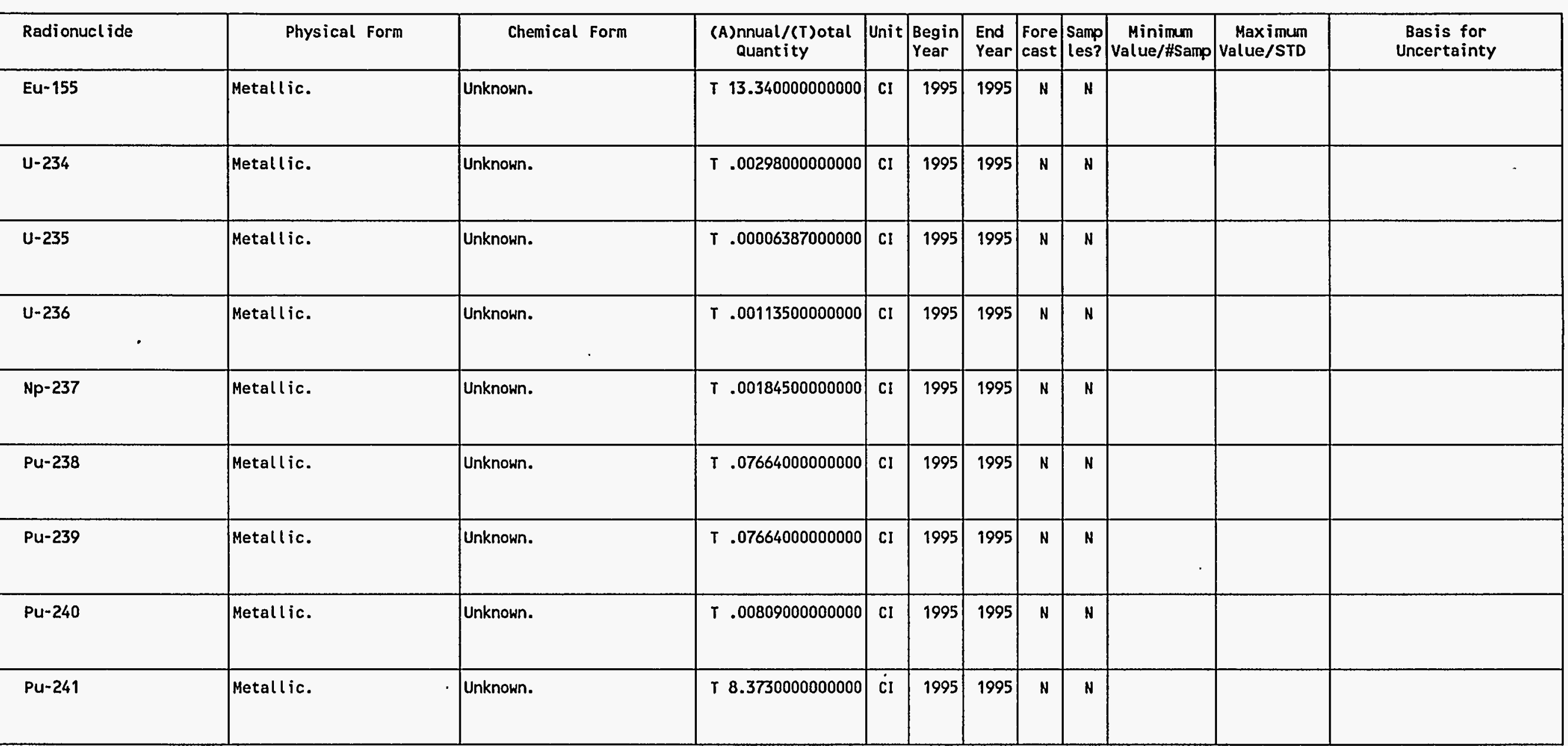

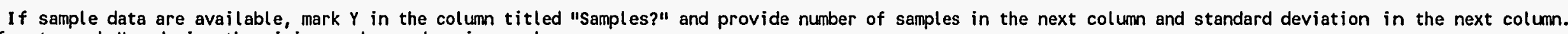
not, mark $N$ and give the minimum value and maximum value.

dditional information or explanations (indicate pertinent contaminant) 
each contaminant, complete at least one line on the following table. If any entries for that contaminant vary by year, fill out additional lines as needed

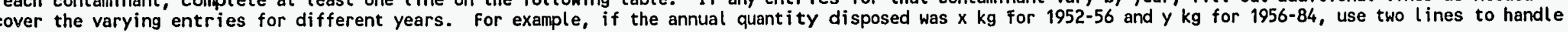
situation.

\begin{tabular}{|c|c|c|c|c|c|c|c|c|c|c|c|}
\hline Radionucl ide & Physical Form & Chemical form & $\begin{array}{l}\text { (A)nnual/( } T \text { )otal } \\
\text { Quant ity }\end{array}$ & Unit & $\begin{array}{l}\text { Begin } \\
\text { Year }\end{array}$ & $\begin{array}{l}\text { End } \\
\text { Year }\end{array}$ & $\begin{array}{l}\text { Fore } \\
\text { cast }\end{array}$ & $\begin{array}{l}\text { Samp } \\
\text { les? }\end{array}$ & $\begin{array}{c}\text { Minimum } \\
\text { Value/\#Samp }\end{array}$ & $\begin{array}{r}\text { Maximum } \\
\text { value/STD }\end{array}$ & $\begin{array}{l}\text { Basis for } \\
\text { Uncertainty }\end{array}$ \\
\hline Am-241 & Metallic. & Unknown. & T. .03832000000000 & CI & 1995 & 1995 & N & N & & & \\
\hline $\mathrm{Cm}-242$ & Metallic. & Unknown. & T. .03832000000000 & CI & 1995 & 1995 & N & N & & & \\
\hline $\mathrm{Cm}-244$ & Metallic. & Unknown. & T. .0354800000000 & CI & 1995 & 1995 & N & N & & & \\
\hline$H-3$ & Metallic. & Unknown. & T 116.40000000000 & $\mathrm{CI}$ & 1996 & 1996 & N & N & & & \\
\hline$C-14$ & Metallic. & Unknown. & $T 15.610000000000$ & $\mathrm{Cl}$ & 1996 & 1996 & N & N & & & \\
\hline $\mathrm{Fe}-55$ & Metallic. & Unknown. & T 2697.0000000000 & $\mathrm{CI}$ & 1996 & 1996 & N & N & & & \\
\hline $\mathrm{Ni}-59$ & Metallic. & Unknown. & T. .8090000000000 & CI & 1996 & 1996 & N & N & & & \\
\hline $\mathrm{Ni}-63$ & Metallic. & Unknown. & T 454.20000000000 & $\mathrm{CI}$ & 1996 & 1996 & N & N & & & \\
\hline Co-60 & Metallic. & Unknown. & T 950.9000000000 & CI & 1996 & 1996 & N & N & & & \\
\hline
\end{tabular}

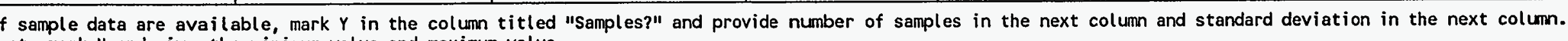
not, mark $N$ and give the minimum value and maximum value.

itional information or explanations (indicate pertinent contaminant) 


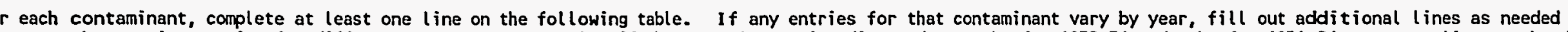

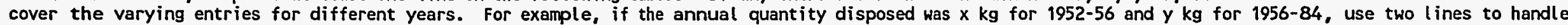
is situation.

\begin{tabular}{|c|c|c|c|c|c|c|c|c|c|c|c|}
\hline Radionucl ide & Physical Form & Chemical form & $\begin{array}{l}\text { (A)nnual/(T)otal } \\
\text { Quantity }\end{array}$ & Unit & $\begin{array}{l}\text { Begin } \\
\text { Year }\end{array}$ & $\begin{array}{l}\text { End } \\
\text { Year }\end{array}$ & $\begin{array}{l}\text { Fore } \\
\text { cast }\end{array}$ & $\begin{array}{l}\text { Samp } \\
\text { les? }\end{array}$ & $\begin{array}{c}\text { Minimum } \\
\text { value/\#Samp }\end{array}$ & $\begin{array}{r}\text { Maximum } \\
\text { Value/STD }\end{array}$ & $\begin{array}{l}\text { Basis for } \\
\text { Uncertainty }\end{array}$ \\
\hline Sr-90 & Metallic. & Unknown. & T 1.3060000000000 & CI & 1996 & 1996 & N & N & & & \\
\hline $\begin{array}{l}\text { Tc-99 } \\
\text {. }\end{array}$ & Metallic. & Unknown. & T.25550000000000 & CI & 1996 & 1996 & N & N & & & \\
\hline I - 129 & Metallic. & Unknown. & $T .00006642000000$ & $\mathrm{CI}$ & 1996 & 1996 & N & N & & & \\
\hline Cs -137 & Metallic. & Unknown. & T 283.80000000000 & $\mathrm{Cl}$ & 1996 & 1996 & N & N & & & \\
\hline $\mathrm{Ce}-144$ & Metallic. & Unknown. & T 6.6700000000000 & CI & 1996 & 1996 & N & N & & & \\
\hline Eu-154 & Metallic. & Unknown. & $T .00411600000000$ & $\mathrm{CI}$ & 1996 & 1996 & N & N & & & \\
\hline Eu-155 & Metallic. & Unknown. & T 13.340000000000 & CI & 1996 & 1996 & N & N & & & \\
\hline$U-234$ & Metallic. & Unknown. & T. .00298000000000 & CI & 1996 & 1996 & N & N & & & \\
\hline$U-235$ & Metallic. & Unknown. & $T .00006387000000$ & CI & 1996 & 1996 & N & N & & & \\
\hline
\end{tabular}

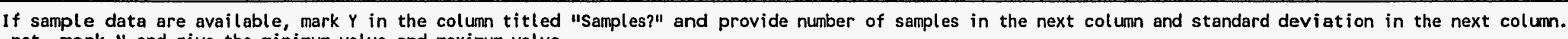
not, mark $N$ and give the minimum value and maximum value.

ditional information or explanations (indicate pertinent contaminant) 
each contaminant, complete at least one line on the following table. If any entries for that contaminant vary by year, fill out additional lines as needed

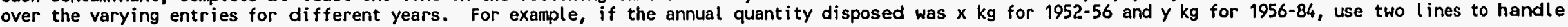
situation.

\begin{tabular}{|c|c|c|c|c|c|c|c|c|c|c|c|}
\hline Radionuclide & Physical form & Chemical Form & $\begin{array}{l}\text { (A)nnual/(T)otal } \\
\text { Quantity }\end{array}$ & Unit & $\begin{array}{l}\text { Begin } \\
\text { Year }\end{array}$ & $\begin{array}{l}\text { End } \\
\text { Year }\end{array}$ & $\begin{array}{l}\text { Fore } \\
\text { cast }\end{array}$ & $\begin{array}{l}\text { Samp } \\
\text { les? }\end{array}$ & $\begin{array}{c}\text { Minimum } \\
\text { Value/\#Samp }\end{array}$ & $\begin{array}{l}\text { Maximum } \\
\text { Value/STD }\end{array}$ & $\begin{array}{l}\text { Basis for } \\
\text { Uncertainty }\end{array}$ \\
\hline$U-236$ & Metallic. & Unknown. & T. .00113500000000 & CI & 1996 & 1996 & N & N & & & \\
\hline Np-237 & Metal L ic. & Unknown. & T. .00184500000000 & CI & 1996 & 1996 & N & N & & & \\
\hline Pu-238 & Metallic. & Unknown. & $T .07664000000000$ & CI & 1996 & 1996 & N & N & & & \\
\hline Pu-239 & Metallic. & Unknown. & T. .07664000000000 & CI & 1996 & 1996 & N & N & & & \\
\hline Pu-240 & Metal l ic. & Unknown. & $T .00809000000000$ & CI & 1996 & 1996 & N & N & & & \\
\hline Pu-241 & Metall ic. & Unknown. & T 8.3730000000000 & CI & 1996 & 1996 & N & N & & & \\
\hline Am-241 & Metall ic. & Unknown. & $T .03832000000000$ & CI & 1996 & 1996 & N & $N$ & & & \\
\hline $\mathrm{Cm}-242$ & Metallic. & Unknown. & $T .03832000000000$ & $\mathrm{CI}$ & 1996 & 1996 & N & H & & & \\
\hline $\mathrm{Cm}-244$ & Metallic. & Unknown. & T. .03548000000000 & CI & 1996 & 1996 & N & N & & & \\
\hline
\end{tabular}

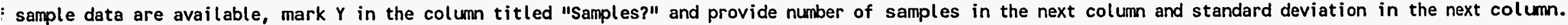

1ot, mark $N$ and give the minimum value and maximum value.

itional information or explanations (indicate pertinent contaminant) 
each contaminant, complete at least one line on the following table. If any entries for that contaminant vary by year, fill out additional lines as needed over the varying entries for different years. For example, if the annual quantity disposed was $x \mathrm{~kg}$ for 1952-56 and y $\mathrm{kg}$ for $1956-84$, use two lines to handle situation.

\begin{tabular}{|c|c|c|c|c|c|c|c|c|c|c|c|}
\hline Radionucl ide & Physical Form & Chemical Form & $\begin{array}{l}\text { (A)nnual /(T) otal } \\
\text { Quantity }\end{array}$ & Unit & $\begin{array}{l}\text { Begin } \\
\text { Year }\end{array}$ & $\begin{array}{l}\text { End } \\
\text { Year }\end{array}$ & $\begin{array}{l}\text { Fore } \\
\text { cast }\end{array}$ & \begin{tabular}{|l|} 
Samp \\
les?
\end{tabular} & $\begin{array}{c}\text { Minimum } \\
\text { Value/\#Samp }\end{array}$ & $\begin{array}{r}\text { Maximum } \\
\text { Value/STD }\end{array}$ & $\begin{array}{l}\text { Basis for } \\
\text { Uncertainty }\end{array}$ \\
\hline Cs- 137 & Metallic. & Unknown. & T 283.80000000000 & CI & 1997 & 1997 & N & N & & & \\
\hline $\mathrm{Ce}-144$ & Metallic. & Unknown. & T 6.6700000000000 & Cl & 1997 & 1997 & $\mathrm{~N}$ & N & & & \\
\hline Eu- 154 & Metallic. & Unknown. & T. .00411600000000 & CI & 1997 & 1997 & $\mathbf{N}$ & N & & & \\
\hline$E u-155$ & Metallic. & Unknown. & T 13.340000000000 & $\mathrm{Cl}$ & 1997 & 1997 & N & N & & & \\
\hline$U-234$ & Metallic. & Unknown. & T. .00298000000000 & CI & 1997 & 1997 & N & N & & & . \\
\hline$U-235$ & Metallic. & Unknown. & $T .00006387000000$ & CI & 1997 & 1997 & $\mathrm{~N}$ & N & & & \\
\hline$U-236$ & Metallic. & Unknown. & $T .00113500000000$ & $\mathrm{CI}$ & 1997 & 1997 & N & N & & & \\
\hline Np-237 & Metallic. & Unknown. & T. .00184500000000 & CI & 1997 & 1997 & N & N & & & \\
\hline Pu-238 & Metallic. & Unknown. & T. .07664000000000 & CI & 1997 & 1997 & N & N & & & \\
\hline
\end{tabular}

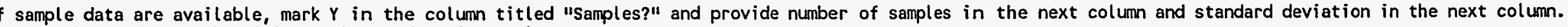

ot, mark $N$ and give the minimum value and maximum value.

itional information or explanations (indicate pertinent contaminant) 
- each contaminant, complete at least one line on the following table. If any entries for that contaminant vary by year, fill out additional lines as needed

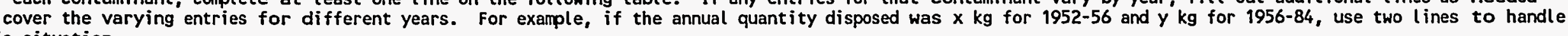
s situation.

\begin{tabular}{|c|c|c|c|c|c|c|c|c|c|c|c|}
\hline Radionuclide & Physical Form & Chemical Form & $\begin{array}{l}\text { (A)nnual/(T)otal } \\
\text { Quantity }\end{array}$ & Unit & $\begin{array}{l}\text { Begin } \\
\text { Year }\end{array}$ & $\begin{array}{l}\text { End } \\
\text { Year }\end{array}$ & $\begin{array}{l}\text { Fore } \\
\text { cast }\end{array}$ & $\begin{array}{l}\text { Samp } \\
\text { les? }\end{array}$ & $\begin{array}{c}\text { Minimum } \\
\text { Value/\#Samp }\end{array}$ & $\begin{array}{r}\text { Maximum } \\
\text { Value/STD }\end{array}$ & $\begin{array}{l}\text { Basis for } \\
\text { Uncertainty }\end{array}$ \\
\hline Pu-239 & Metallic. & Unknown. & $T .07664000000000$ & $\mathbf{C I}$ & 1997 & 1997 & N & H & & & \\
\hline Pu-240 & Metallic. & Unknown. & $T .00809000000000$ & CI & 1997 & 1997 & N & N & & & \\
\hline Pu-241 & Metallic. & Unknown. & T 8.3730000000000 & Cl & 1997 & 1997 & N & N & & & \\
\hline$A m-241$ & Metall ic. & Unknown. & T .03832000000000 & CI & 1997 & 1997 & N & N & & & \\
\hline $\mathrm{Cm}-242$ & Metallic. & Unknown. & $T .03832000000000$ & CI & 1997 & 1997 & N & N & & & \\
\hline $\mathrm{Cm}-244$ & Metallic. & Unknown. & T. .03548000000000 & CI & 1997 & 1997 & N & N & & & \\
\hline$H-3$ & Metallic. & Unknown. & T 116.40000000000 & CI & 1998 & 1998 & N & N & & & \\
\hline$C-14$ & Metallic. & Unknown. & T 15.610000000000 & CI & 1998 & 1998 & N & N & & & \\
\hline $\mathrm{Fe}-55$ & Metallic. & Unknown. & T 2697.0000000000 & CI & 1998 & 1998 & N & N & & & \\
\hline
\end{tabular}

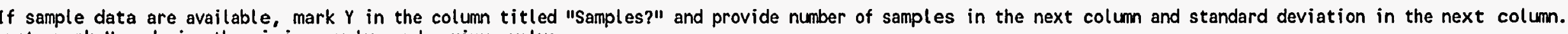
not, mark $N$ and give the minimum value and maximum value.

ditional information or explanations (indicate pertinent contaminant) 
ach contaminant, complete at least one line on the following table. If any entries for that contaminant vary by year, fill out additional lines as needed ver the varying entries for different years. For example, if the annual quantity disposed was $x \mathrm{~kg}$ for 1952-56 and $y$ kg for $1956-84$, use two lines to handle situation.

\begin{tabular}{|c|c|c|c|c|c|c|c|c|c|c|c|}
\hline adionuclide & Physical Form & Chemical form & $\begin{array}{l}\text { (A)nnual /(T)otal } \\
\text { Quant ity }\end{array}$ & Unit & $\begin{array}{l}\text { Begin } \\
\text { Year }\end{array}$ & $\begin{array}{l}\text { End } \\
\text { Year }\end{array}$ & $\begin{array}{l}\text { Fore } \\
\text { cast }\end{array}$ & $\begin{array}{l}\text { Samp } \\
\text { les? }\end{array}$ & $\begin{array}{c}\text { Minimum } \\
\text { Value/\#Samp }\end{array}$ & $\begin{array}{r}\text { Maximum } \\
\text { value/STD }\end{array}$ & $\begin{array}{l}\text { Basis for } \\
\text { Uncertainty }\end{array}$ \\
\hline i-59 & Metallic. & Unknown. & $r .80900000000000$ & CI & 1998 & 1998 & $\mathbf{N}$ & $N$ & & & \\
\hline$i-63$ & Metallic. & Unknown. & $T 454.20000000000$ & CI & 1998 & 1998 & N & H & & & \\
\hline $0-60$ & Metallic. & Unknown. & T 950.90000000000 & CI & 1998 & 1998 & N & N & & & \\
\hline$r-90$ & Metallic. & Unknown. & T 1.3060000000000 & CI & 1998 & 1998 & $\mathbf{N}$ & N & & & \\
\hline$c-99$ & Metallic. & Unknown. & T. .25550000000000 & CI & 1998 & 1998 & N & N & & & \\
\hline-129 & Metallic. & Unknown. & T. .00006642000000 & CI & 1998 & 1998 & N & N & & & \\
\hline s-137 & Metallic. & Unknown. & $T 283.80000000000$ & CI & 1998 & 1998 & N & $N$ & & & \\
\hline$e-144$ & Metallic. & Unknown. & T 6.6700000000000 & CI & 1998 & 1998 & N & N & & & \\
\hline$u-154$ & Metallic. & Unknown. & T. .00411600000000 & $\mathrm{CI}$ & 1998 & 1998 & $\mathbf{N}$ & $N$ & & & \\
\hline
\end{tabular}

sample data are available, mark $Y$ in the column titled "Samples?" and provide number of samples in the next column and standard deviation in the next column.

st, mark $N$ and give the minimum value and maximum value.

ional information or explanations (indicate pertinent contaminant) 
$r$ each contaminant, complete at least one line on the following table. If any entries for that contaminant vary by year, fill out additional lines as needed

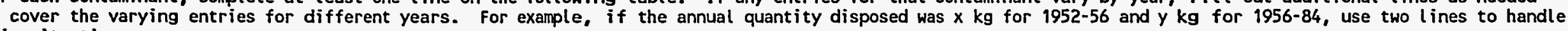
is situation.

\begin{tabular}{|c|c|c|c|c|c|c|c|c|c|c|c|}
\hline Radionuclide & Physical Form & Chemical Form & $\begin{array}{l}\text { (A)nnual/(T)otal } \\
\text { Quantity }\end{array}$ & Unit & $\begin{array}{l}\text { Begin } \\
\text { Year }\end{array}$ & $\begin{array}{l}\text { End } \\
\text { Year }\end{array}$ & $\begin{array}{l}\text { Fore } \\
\text { cast }\end{array}$ & $\begin{array}{l}\text { Samp } \\
\text { les? }\end{array}$ & $\underset{\text { Malue/\#Samp }}{\text { Minimum }}$ & $\begin{array}{c}\text { Maximum } \\
\text { Value/STD }\end{array}$ & $\begin{array}{l}\text { Basis for } \\
\text { Uncertainty }\end{array}$ \\
\hline Eu-155 & Metallic. & Unknown. & T 13.340000000000 & CI & 1998 & 1998 & N & N & & & \\
\hline$U-234$ & Metallic. & Unknown. & $T .00298000000000$ & $\mathrm{Cl}$ & 1998 & 1998 & N & N & & & \\
\hline$U-235$ & Metallic. & Unknown. & $T .00006387000000$ & CI & 1998 & 1998 & N & N & & & \\
\hline$U-236$ & Metallic. & Unknown. & T. .00113500000000 & $\mathrm{CI}$ & 1998 & 1998 & N & N & & & \\
\hline$N p-237$ & Metallic. & Unknown. & $T .00184500000000$ & CI & 1998 & 1998 & N & N & & & \\
\hline Pu-238 & Metallic. & Unknown. & $T .07664000000000$ & CI & 1998 & 1998 & $\mathbf{N}$ & N & & & \\
\hline Pu-239 & Metallic. & Unknown. & $T .07664000000000$ & $\mathrm{Cl}$ & 1998 & 1998 & $\mathbf{N}$ & N & & & \\
\hline$P u-240$ & Metallic. & Unknown. & $T .00809000000000$ & CI & 1998 & 1998 & N & N & & & \\
\hline Pu-241 & Metallic. & Unknown. & T 8.3730000000000 & CI & 1998 & 1998 & N & $\mathbf{N}$ & & & \\
\hline
\end{tabular}

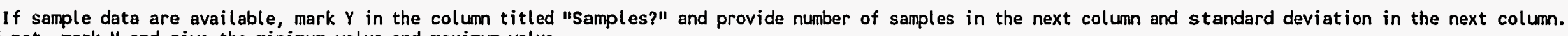
not, mark $N$ and give the minimum value and maximum value.

ditional information or explanations (indicate pertinent contaminant) 
ach contaminant, complete at least one line on the following table. If any entries for that contaminant vary by year, fill out additional lines as needed ver the varying entries for different years. For example, if the annual quantity disposed was $x \mathrm{~kg}$ for $1952-56$ and $y$ kg for $1956-84$, use two lines to handle situation.

\begin{tabular}{|c|c|c|c|c|c|c|c|c|c|c|c|}
\hline adionuclide & Physical Form & Chemical Form & $\begin{array}{l}\text { (A)nnual/(T)otal } \\
\text { Quantity }\end{array}$ & Unit & $\begin{array}{l}\text { Begin } \\
\text { Year }\end{array}$ & $\begin{array}{l}\text { End } \\
\text { Year }\end{array}$ & $\begin{array}{l}\text { Fore } \\
\text { cast }\end{array}$ & $\begin{array}{l}\text { Samp } \\
\text { les? }\end{array}$ & $\begin{array}{c}\text { Minimum } \\
\text { Value/\#Samp }\end{array}$ & $\begin{array}{c}\text { Maximum } \\
\text { Value/STD }\end{array}$ & $\begin{array}{l}\text { Basis for } \\
\text { Uncertainty }\end{array}$ \\
\hline$m-241$ & Metallic. & Unknown. & T. .03832000000000 & CI & 1998 & 1998 & N & N & & & \\
\hline$m-242$ & Metallic. & Unknown. & $T .03832000000000$ & CI & 1998 & 1998 & N & N & & & \\
\hline$m-244$ & Metallic. & Unknown. & $T .03548000000000$ & $\mathrm{CI}$ & 1998 & 1998 & N & $N$ & & & \\
\hline-3 & Metallic. & Unknown. & T 116.40000000000 & CI & 1999 & 1999 & N & $N$ & & & \\
\hline-14 & Metallic. & Unknown. & T 15.610000000000 & CI & 1999 & 1999 & N & N & & & \\
\hline$e-55$ & Metallic. & Unknown. & T 2697.0000000000 & CI & 1999 & 1999 & N & $N$ & & & \\
\hline i-59 & Metallic. & Unknown. & T. .80900000000000 & $\mathrm{ci}$ & 1999 & 1999 & N & $N$ & & & \\
\hline $1 i-63$ & Metallic. & Unknown. & T 454.20000000000 & $\mathrm{Cl}$ & 1999 & 1999 & N & N & & & \\
\hline $0-60$ & Metallic. & Unknown. & T 950.90000000000 & $\mathrm{CI}$ & 1999 & 1999 & N & N & & & \\
\hline
\end{tabular}

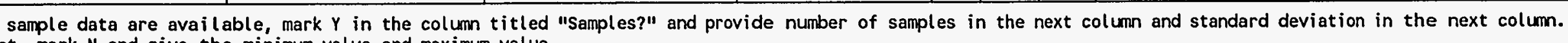
ot, mark $N$ and give the minimum value and maximum value.

tional information or explanations (indicate pertinent contaminant) 
each contaminant, complete at least one line on the following table. If any entries for that contaminant vary by year, fill out additional lines as needed

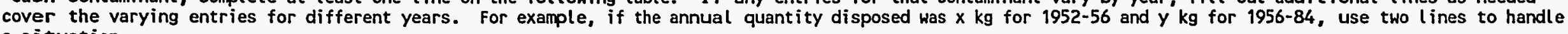
s situation.

\begin{tabular}{|c|c|c|c|c|c|c|c|c|c|c|c|}
\hline Radionucl ide & Physical Form & Chemical form & $\begin{array}{l}\text { (A)nnual/(T)otal } \\
\text { Quantity }\end{array}$ & Unit & $\begin{array}{l}\text { Begin } \\
\text { Year }\end{array}$ & $\begin{array}{l}\text { End } \\
\text { Year }\end{array}$ & $\begin{array}{l}\text { Fore } \\
\text { cast }\end{array}$ & $\begin{array}{l}\text { Samp } \\
\text { les? }\end{array}$ & $\begin{array}{l}\text { Minimum } \\
\text { Value/\#Samp }\end{array}$ & $\begin{array}{r}\text { Maximum } \\
\text { Value/STD }\end{array}$ & $\begin{array}{l}\text { Basis for } \\
\text { Uncertainty }\end{array}$ \\
\hline $\mathrm{Sr}-90$ & Metallic. & Unknown. & T 1.3060000000000 & CI & 1999 & 1999 & $\mathbf{N}$ & $\mathbf{N}$ & & & \\
\hline Tc-99 & Metallic. & Unknown. & T.25550000000000 & $\mathrm{CI}$ & 1999 & 1999 & N & $\mathbf{N}$ & & & \\
\hline $1-129$ & Metallic. & Unknown. & T. .00006642000000 & CI & 1999 & 1999 & N & N & & & \\
\hline Cs-137 & Metallic. & Unknown. & T 283.80000000000 & CI & 1999 & 1999 & $\mathbf{N}$ & N & & & \\
\hline $\mathrm{Ce}-144$ & Metallic. & Unknown. & T 6.6700000000000 & CI & 1999 & 1999 & N & N & & & \\
\hline Eu- 154 & Metallic. & Unknown. & T.00411600000000 & $\mathrm{Cl}$ & 1999 & 1999 & $N$ & N & & & \\
\hline Eu-155 & Metallic. & Unknown. & T 13.340000000000 & $\mathrm{Cl}$ & 1999 & 1999 & $\mathbf{N}$ & $\mathbf{N}$ & & & \\
\hline$U-234$ & Metallic. & Unknown. & T .00298000000000 & CI & 1999 & 1999 & N & N & & & \\
\hline$U-235$ & Metallic. & Unknown. & $T .00006387000000$ & CI & 1999 & 1999 & N & N & & & \\
\hline
\end{tabular}

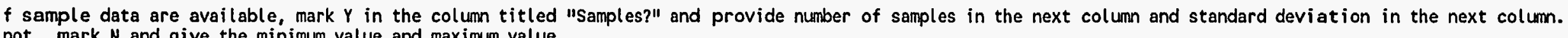
not, mark $N$ and give the minimum value and maximum value.

litional information or explanations (indicate pertinent contaminant) 
each contaminant, complete at least one line on the following table. If any entries for that contaminant vary by year, fill out additional lines as needed

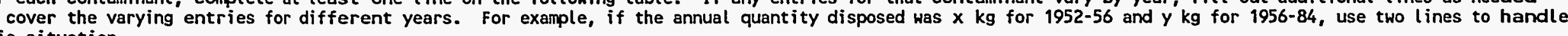
is situation.

\begin{tabular}{|c|c|c|c|c|c|c|c|c|c|c|c|}
\hline Radionuclide & Physical Form & Chemical Form & $\begin{array}{l}\text { (A)nnual/(T)otal } \\
\text { Quantity }\end{array}$ & Unit & $\begin{array}{l}\text { Begin } \\
\text { Year }\end{array}$ & $\begin{array}{l}\text { End } \\
\text { Year }\end{array}$ & $\begin{array}{l}\text { Fore } \\
\text { cast }\end{array}$ & $\begin{array}{l}\text { Samp } \\
\text { les? }\end{array}$ & $\begin{array}{c}\text { Minimum } \\
\text { Value/\#Samp }\end{array}$ & $\begin{array}{r}\text { Maximum } \\
\text { Value/STD }\end{array}$ & $\begin{array}{l}\text { Basis for } \\
\text { Uncertainty }\end{array}$ \\
\hline$H-3$ & Metallic. & Unknown. & T 116.40000000000 & CI & 2000 & 2000 & N & N & & & \\
\hline$c-14$ & Metallic. & Unknown. & T 15.610000000000 & CI & 2000 & 2000 & N & N & & & \\
\hline $\mathrm{Fe}-55$ & Metallic. & Unknown. & T 2697.0000000000 & CI & 2000 & 2000 & N & N & & & \\
\hline $\mathrm{Ni}-59$ & Metallic. & Unknown. & $T .80900000000000$ & CI & 2000 & 2000 & N & N & & & \\
\hline $\mathrm{Ni}-63$ & Metallic. & Unknown. & T 454.20000000000 & CI & 2000 & 2000 & N & N & & & \\
\hline Co-60 & Metallic. & Unknown. & T 950.90000000000 & CI & 2000 & 2000 & N & $\mathbf{N}$ & & & \\
\hline $\mathrm{sr}-90$ & Metallic. & Unknown. & $T 1.3060000000000$ & CI & 2000 & 2000 & N & N & & & \\
\hline Tc-99 & Metallic. & Unknown. & T.25550000000000 & CI & 2000 & 2000 & N & N & & & \\
\hline $1-129$ & Metallic. & Unknown. & T .00006642000000 & CI & 2000 & 2000 & N & N & & & \\
\hline
\end{tabular}

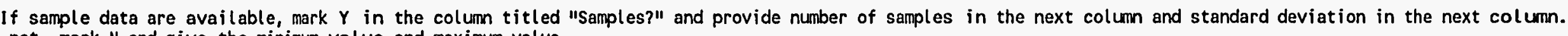
not, mark $N$ and give the minimum value and maximum value.

ditional information or explanations (indicate pertinent contaminant) 
each contaminant, complete at least one line on the following table. If any entries for that contaminant vary by year, fill out additional lines as needed over the varying entries for different years. For example, if the annual quantity disposed was $x \mathrm{~kg}$ for $1952-56$ and $y$ kg for $1956-84$, use two lines to handle si tuation.

\begin{tabular}{|c|c|c|c|c|c|c|c|c|c|c|c|}
\hline Radionucl ide & Physical form & Chemical Form & $\begin{array}{l}\text { (A)nnual/( } T \text { )otal } \\
\text { Quantity }\end{array}$ & Unit & $\begin{array}{l}\text { Begin } \\
\text { Year }\end{array}$ & $\begin{array}{l}\text { End } \\
\text { Year }\end{array}$ & $\begin{array}{l}\text { Fore } \\
\text { cast }\end{array}$ & $\begin{array}{l}\text { Samp } \\
\text { Les? }\end{array}$ & $\begin{array}{c}\text { Minimum } \\
\text { Value/\#Samp }\end{array}$ & $\begin{array}{c}\text { Maximum } \\
\text { Value/Sto }\end{array}$ & $\begin{array}{l}\text { Basis for } \\
\text { Uncertainty }\end{array}$ \\
\hline$C s-137$ & Metallic. & Unknown. & T 283.80000000000 & $\mathrm{Cl}$ & 2000 & 2000 & N & N & & & \\
\hline Ce-144 & Metallic. & Unknown. & T 6.6700000000000 & $\mathrm{Cl}$ & 2000 & 2000 & H & N & & & \\
\hline Eu-154 & Metallic. & Unknown. & $T .00411600000000$ & $\mathrm{CI}$ & 2000 & 2000 & N & $N$ & & & \\
\hline Eu-155 & Metallic. & Unknown. & T 13.340000000000 & $\mathrm{CI}$ & 2000 & 2000 & N & N & & & \\
\hline$U-234$ & Metallic. & Unknown. & $T .00298000000000$ & CI & 2000 & 2000 & N & N & & & \\
\hline$U-235$ & Metallic. & Unknown. & $T .00006387000000$ & CI & 2000 & 2000 & N & N & & & \\
\hline$U-236$ & Metallic. & Unknown. & T. .00113500000000 & CI & 2000 & 2000 & N & N & & & \\
\hline Np-237 & Metallic. & Unknown. & $T .00184500000000$ & CI & 2000 & 2000 & N & N & & & \\
\hline Pu-238 & Metallic. & Unknown. & T. .07664000000000 & $\mathrm{CI}$ & 2000 & 2000 & N & $\mathbf{N}$ & & & \\
\hline
\end{tabular}

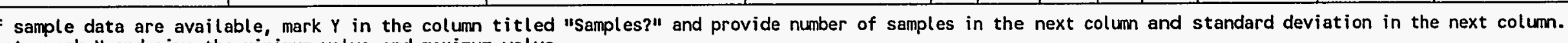
10t, mark $N$ and give the minimum value and maximum value.

tional information or explanations (indicate pertinent contaminant) 
zach contaminant, complete at least one line on the following table. If any entries for that contaminant vary by year, fill out additional lines as needed sver the varying entries for different years. For example, if the annual quantity disposed was $x \mathrm{~kg}$ for 1952-56 and y $\mathrm{kg}$ for $1956-84$, use two lines to handle situation.

\begin{tabular}{|c|c|c|c|c|c|c|c|c|c|c|c|}
\hline Radionucl ide & Physical Form & Chemical form & $\begin{array}{l}\text { (A)nnual } /(T) \text { otal } \\
\text { Quant ity }\end{array}$ & Unit & $\begin{array}{l}\text { Begin } \\
\text { Year }\end{array}$ & $\begin{array}{l}\text { End } \\
\text { Year }\end{array}$ & $\begin{array}{l}\text { Fore } \\
\text { cast }\end{array}$ & $\begin{array}{l}\text { Samp } \\
\text { les? }\end{array}$ & $\begin{array}{c}\text { Minimum } \\
\text { value/\#Samp }\end{array}$ & $\begin{array}{r}\text { Maximum } \\
\text { Value/STD }\end{array}$ & $\begin{array}{l}\text { Bas is for } \\
\text { Uncertainty }\end{array}$ \\
\hline $1 i-59$ & Metallic. & Unknown. & $T .80900000000000$ & $\mathrm{CI}$ & 2001 & 2001 & N & $\mathbf{N}$ & & & \\
\hline$v i-63$ & Metallic. & Unknown. & T 454.20000000000 & CI & 2001 & 2001 & N & $\mathbf{N}$ & & & \\
\hline $50-60$ & Metallic. & Unknown. & T 950.90000000000 & CI & 2001 & 2001 & $\mathbf{N}$ & N & & & \\
\hline $3 r-90$ & Metallic. & Unknown. & T 1.3060000000000 & CI & 2001 & 2001 & N & N & & & \\
\hline Tc-99 & Metallic. & Unknown. & T.25550000000000 & $\mathrm{Cl}$ & 2001 & 2001 & $\mathbf{N}$ & N & & & \\
\hline $1-129$ & Metallic. & Unknown. & $T .00006642000000$ & Cl & 2001 & 2001 & H & N & & & \\
\hline Cs -137 & Metallic. & Unknown. & T 283.80000000000 & CI & 2001 & 2001 & N & N & & & \\
\hline Ce- 144 & Metallic. & Unknown. & T 6.6700000000000 & $\mathrm{CI}$ & 2001 & 2001 & $\mathrm{~N}$ & N & & & \\
\hline Eu-154 & Metallic. & Unknown. & T. .00411600000000 & $\mathrm{Cl}$ & 2001 & 2001 & $\mathbf{N}$ & $N$ & & & \\
\hline
\end{tabular}

sample data are available, mark $Y$ in the column titled "Samples?" and provide number of samples in the next column and standard deviation in the next column. ot, mark $\mathrm{N}$ and give the minimum value and maximum value.

tional information or explanations (indicate pertinent contaminant) 
or each contaminant, complete at least one line on the following table. If any entries for that contaminant vary by year, fill out additional lines as needed

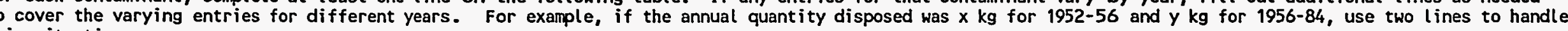
is situation.

\begin{tabular}{|c|c|c|c|c|c|c|c|c|c|c|c|}
\hline Radionucl ide & Physical Form & Chemical Form & $\begin{array}{l}\text { (A)nnual/(T)otal } \\
\text { Quantity }\end{array}$ & Unit & $\begin{array}{l}\text { Begin } \\
\text { Year }\end{array}$ & $\begin{array}{l}\text { End } \\
\text { Year }\end{array}$ & $\begin{array}{l}\text { Fore } \\
\text { cast }\end{array}$ & $\begin{array}{l}\text { Samp } \\
\text { les? }\end{array}$ & $\underset{\text { Malue/\#Samp }}{\text { Minimum }}$ & $\begin{array}{c}\text { Maximum } \\
\text { Value/STD }\end{array}$ & $\begin{array}{l}\text { Basis for } \\
\text { Uncertainty }\end{array}$ \\
\hline Eu-155 & Metallic. & Unknown. & T 13.340000000000 & CI & 2001 & 2001 & N & N & & & \\
\hline$U-234$ & Metall ic. & Unknown. & T. .00298000000000 & $\mathrm{CI}$ & 2001 & 2001 & N & N & & & \\
\hline$U-235$ & Metallic. & Unknown. & T. .00006387000000 & CI & 2001 & 2001 & N & N & & & \\
\hline$U-236$ & Metallic. & Unknown. & $T .00113500000000$ & CI & 2001 & 2001 & N & N & & & \\
\hline$N p-237$ & Metallic. & Unknown. & $T .00184500000000$ & CI & 2001 & 2001 & N & N & & & \\
\hline$P u-238$ & Metallic. & Unknown. & $T .07664000000000$ & CI & 2001 & 2001 & N & N & & & \\
\hline Pu-239 & Metallic. & Unknown. & $T .07664000000000$ & CI & 2001 & 2001 & N & N & & & \\
\hline Pu-240 & Metallic. & Unknown. & $T .00809000000000$ & CI & 2001 & 2001 & $\mathbf{N}$ & N & & & \\
\hline Pu-241 & Metallic. & Unknown. & T 8.3730000000000 & CI & 2001 & 2001 & $\mathbf{N}$ & $N$ & & & \\
\hline
\end{tabular}

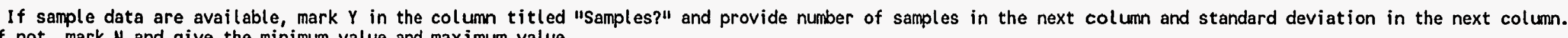

not, mark $N$ and give the minimum value and maximum value.
dditional information or explanations (indicate pertinent contaminant) 


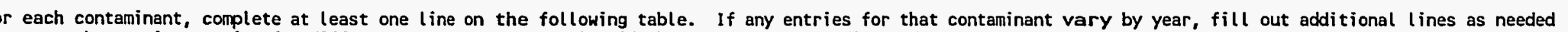

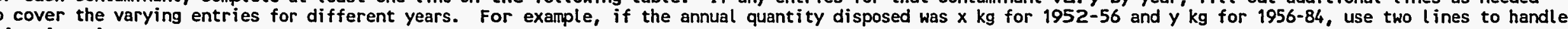
is situation.

\begin{tabular}{|c|c|c|c|c|c|c|c|c|c|c|c|}
\hline Radionucl ide & Physical Form & Chemical form & $\begin{array}{l}\text { (A)nnual/(T)otal } \\
\text { Quantity }\end{array}$ & Unit & $\begin{array}{l}\text { Begin } \\
\text { Year }\end{array}$ & $\begin{array}{l}\text { End } \\
\text { Year }\end{array}$ & $\begin{array}{l}\text { Fore } \\
\text { cast }\end{array}$ & $\begin{array}{l}\text { Samp } \\
\text { les? }\end{array}$ & $\begin{array}{c}\text { Minimum } \\
\text { Value/\#Samp }\end{array}$ & $\begin{array}{r}\text { Maximum } \\
\text { Value/STD }\end{array}$ & $\begin{array}{l}\text { Basis for } \\
\text { Uncertainty }\end{array}$ \\
\hline Sr-90 & Metallic. & Unknown. & T 1.3060000000000 & CI & 2002 & 2002 & N & N & & & \\
\hline Tc-99 & Metallic. & Unknown. & T. .25550000000000 & CI & 2002 & 2002 & N & N & & & \\
\hline$I-129$ & Metallic. & Unknown. & $T .00006642000000$ & CI & 2002 & 2002 & N & N & & & \\
\hline Cs -137 & Metallic. & Unknown. & T 283.80000000000 & CI & 2002 & 2002 & N & N & & & \\
\hline Ce- 144 & Metallic. & Unknown. & T 6.6700000000000 & CI & 2002 & 2002 & N & N & & & \\
\hline Eu-154 & Metallic. & Unknown. & $T .00411600000000$ & CI & 2002 & 2002 & N & N & & & \\
\hline Eu- 155 & Metallic. & Unknown. & T 13.340000000000 & CI & 2002 & 2002 & N & N & & & \\
\hline$U-234$ & Metallic. & Unknown. & $T .00298000000000$ & CI & 2002 & 2002 & N & N & & & \\
\hline$U-235$ & Metallic. & Unknown. & $T .00006387000000$ & CI & 2002 & 2002 & N & N & & & \\
\hline
\end{tabular}

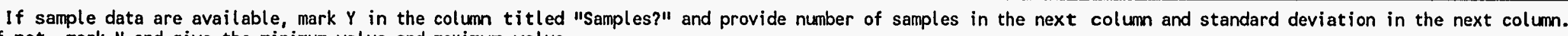
not, mark $N$ and give the minimum value and maximum value.

ditional information or explanations (indicate pertinent contaminant) 
each contaminant, complete at least one line on the following table. If any entries for that contaminant vary by year, fill out additional lines as needed over the varying entries for different years. For example, if the annual quantity disposed was $x \mathrm{~kg}$ for $1952-56$ and $y \mathrm{~kg}$ for $1956-84$, use two lines to handle situation.

\begin{tabular}{|c|c|c|c|c|c|c|c|c|c|c|c|}
\hline ladionucl ide & Physical Form & Chemical form & $\begin{array}{l}\text { (A) nnual/( } T \text { ) otal } \\
\text { Quantity }\end{array}$ & Unit & $\begin{array}{l}\text { Begin } \\
\text { Year }\end{array}$ & $\begin{array}{l}\text { End } \\
\text { Year }\end{array}$ & $\begin{array}{l}\text { Fore } \\
\text { cast }\end{array}$ & $\begin{array}{l}\text { Samp } \\
\text { les? }\end{array}$ & \begin{tabular}{|} 
Minimum \\
Value/\#Samp
\end{tabular} & $\begin{array}{l}\text { Maximum } \\
\text { Value/STD }\end{array}$ & $\begin{array}{l}\text { Basis for } \\
\text { Uncertainty }\end{array}$ \\
\hline j-236 & Metallic. & Unknown. & $T .00113500000000$ & $\mathrm{CI}$ & 2002 & 2002 & N & N & & & \\
\hline$p-237$ & Metallic. & Unknown. & T. .00184500000000 & CI & 2002 & 2002 & N & N & & & \\
\hline$u-238$ & Metallic. & Unknown. & T. .07664000000000 & CI & 2002 & 2002 & N & N & & & \\
\hline$u-239$ & Metallic. & Unknown. & T. .07664000000000 & $\mathrm{Cl}$ & 2002 & 2002 & $N$ & $N$ & & & \\
\hline$u-240$ & Metallic. & Unknown. & T. .00809000000000 & CI & 2002 & 2002 & N & $N$ & & & \\
\hline$u-241$ & Metallic. & Unknown. & T 8.3730000000000 & CI & 2002 & 2002 & $N$ & $N$ & & & \\
\hline $9 m-241$ & Metallic. & Unknown. & T. .03832000000000 & CI & 2002 & 2002 & N & N & & & \\
\hline $\mathrm{Cm}-242$ & Metallic. & Unknown. & $T .03832000000000$ & CI & 2002 & 2002 & N & N & . & & \\
\hline$m-244$ & Metallic. & Unknown. & $T .03548000000000$ & $\mathrm{Cl}$ & 2002 & 2002 & N & $N$ & & & \\
\hline
\end{tabular}

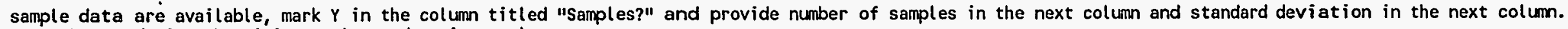

ot, mark $N$ and give the minimum value and maximum value.

tional information or explanations (indicate pertinent contaminant) 


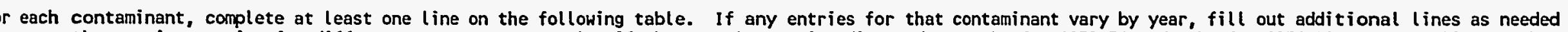

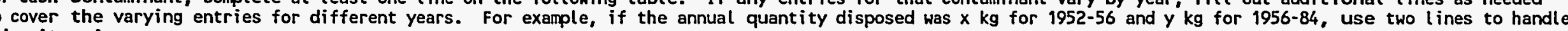
is situation.

\begin{tabular}{|c|c|c|c|c|c|c|c|c|c|c|c|}
\hline Radionucl ide & Physical Form & Chemical Form & $\begin{array}{l}\text { (A)nnual/(T)otal } \\
\text { Quantity }\end{array}$ & Unit & $\begin{array}{l}\text { Begin } \\
\text { Year }\end{array}$ & $\begin{array}{l}\text { End } \\
\text { Year }\end{array}$ & $\begin{array}{l}\text { Fore } \\
\text { cast }\end{array}$ & \begin{tabular}{|l|} 
Samp \\
les?
\end{tabular} & $\begin{array}{c}\text { Minimum } \\
\text { Value/\#Samp }\end{array}$ & $\begin{array}{r}\text { Maximum } \\
\text { Value/STD }\end{array}$ & $\begin{array}{l}\text { Basis for } \\
\text { Uncertainty }\end{array}$ \\
\hline $\mathrm{H}-3$ & Metallic. & Unknown. & T 116.40000000000 & Cl & 2003 & 2003 & N & N & & & \\
\hline$c-14$ & Metallic. & Unknown. & T 15.610000000000 & Cl & 2003 & 2003 & N & N & & & \\
\hline $\mathrm{Fe}-55$ & Metallic. & Unknown. & T 2697.0000000000 & $\mathrm{CI}$ & 2003 & 2003 & N & N & & & \\
\hline $\mathrm{Ni}-59$ & Metallic. & Unknown. & $T .80900000000000$ & CI & 2003 & 2003 & N & N & & & \\
\hline $\mathrm{Ni}-63$ & Metallic. & Unknown. & T 454.20000000000 & $\mathrm{CI}$ & 2003 & 2003 & N & N & & & \\
\hline Co- 60 & Metallic. & Unknown. & T 950.90000000000 & $\mathrm{Cl}$ & 2003 & 2003 & N & N & & & \\
\hline$S r-90$ & Metallic. & Unknown. & T 1.3060000000000 & $\mathrm{CI}$ & 2003 & 2003 & N & N & & & \\
\hline Tc-99 & Metallic. & Unknown. & $T .25550000000000$ & $\mathrm{Cl}$ & 2003 & 2003 & N & N & & & \\
\hline$I-129$ & Hetallic. & Unknown. & $T .00006642000000$ & $\mathrm{Cl}$ & 2003 & 2003 & N & N & & & \\
\hline
\end{tabular}

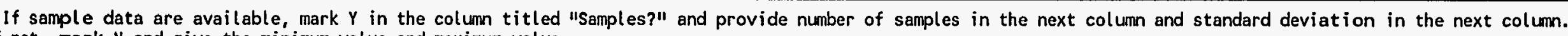
not, mark $N$ and give the minimum value and maximum value.

iditional information or explanations (indicate pertinent contaminant) 
each contaminant, complete at least one line on the following table. If any entries for that contaminant vary by year, fill out additional lines as needed

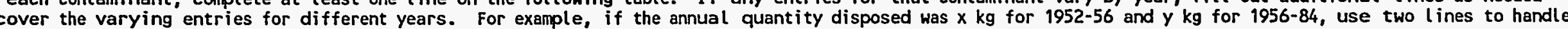
situation.

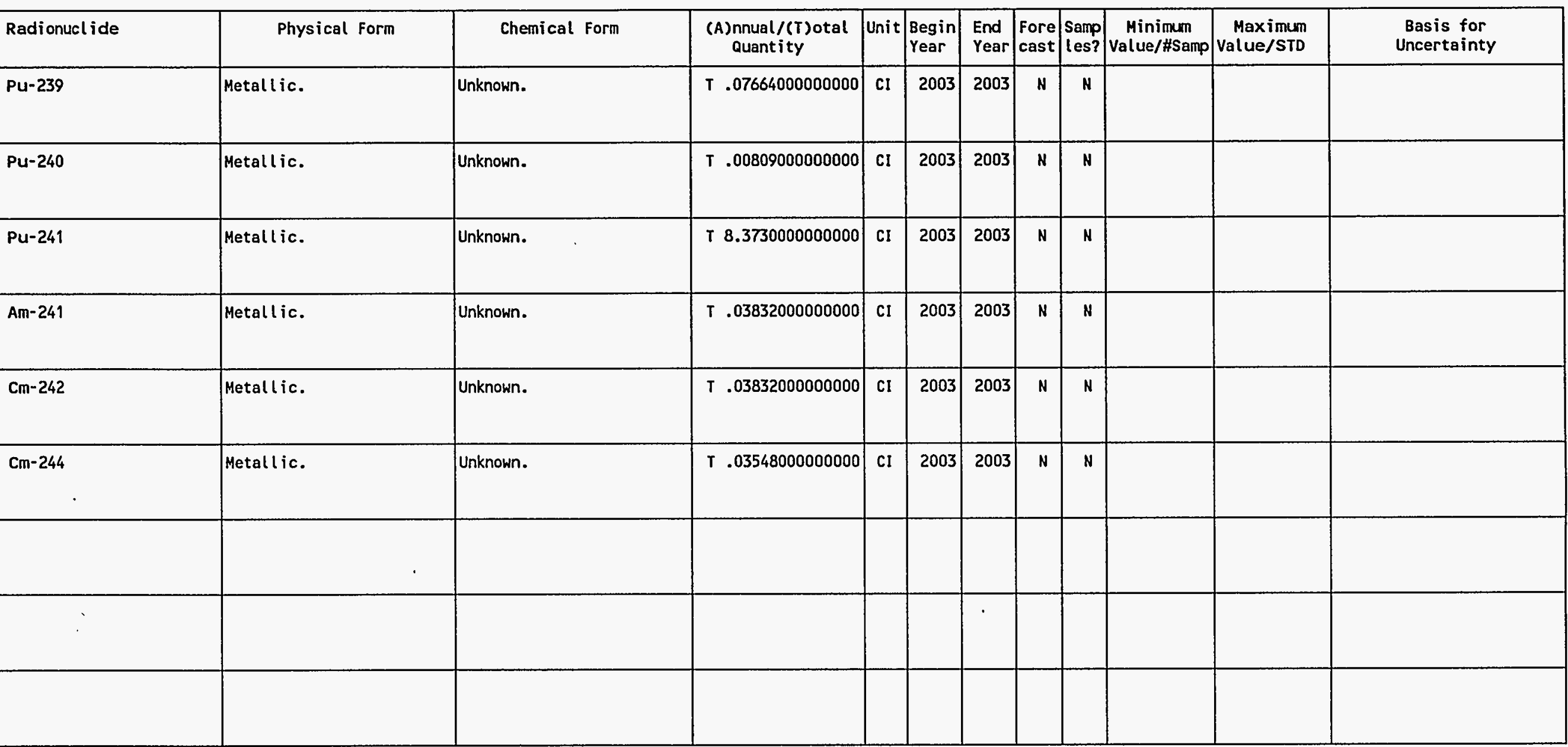

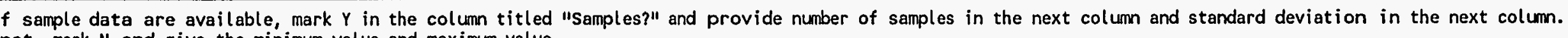
not, mark $N$ and give the minimum value and maximum value.

itional information or explanations (indicate pertinent contaminant) 
Type of source of information: leck box)

RWMIS [ ] other database

sample analysis data

operating records [ ] interview

expert judgment [ ] reports

generator forecasts

other

Do the estimates of contaminant antities in Part $C$ and $D$ represent: best estimate

worst case

other

Do the data conflict with RWMTS? (Historical or Present Data only) no

yes

Major unknowns in inventories of ataminants:

identified beta gamma (U-B-G) was known. The $\mathrm{Ci}$ content was unknown.
2. Details concerning source (names, report no., dates, etc.)

4. If other than best estimate, explain why:

6. If yes, explain why:

A portion of or all of the measurements were assumed to be taken using a G-M detector which only measures gamma emitters; beta emitters have been added to the RWMIS activity based on scaling factors. Therefore, the total. activity will be greater than that in RWMIS.

8. Key assumptions used to deal with the unknowns: U-B-G radionuclides were chosen based on EPR1 NP-5077 for dry active waste for PWRs. PWR was chosen because it is similar to the ATR, the reactor at TRA. The radionuclides chosen were based on a combination of the following: cross sections, half lives and fission products. To determine the Ci content, 1991-93 Ci content was averaged, then multiplied by 10 (for 1994-2003 - 10 years) and this $\mathrm{Ci}$ volume was assigned to the unidentified beta-gamma. 
DATA INPUT FOR HISTORICAL DATA TASK FOR RWMC SUBSURFACE DISPOSAL AREA

\section{ART A - GENERAL INFORMATION PDT - 297}

- Preparer: Amaro, C.

- Generator: TRA

area or contractor - use code from attached list)

- Number of waste stream from this facility:

$15 \mathrm{P}$

- Type of radioactive waste (check box):

] TRU or suspect TRU

X] LLW

] non-radioactive

- Actual years disposed of at SDA: tarting year 1994 Ending year 2003

0 . Comments (specify number of pertinent question): 4. Also probably $632,670,635$ and others.
2. Date prepared: $07 / 16 / 94$

4. Particular facility: 603

(building number - use code from attached list)

6. Waste stream:

Metal, such as aluminum and stainless steel.

9. Waste stream volume:

Amount

85.0000 Units Cubic meters.

Check box: [ ] annual or [X] total over all years

Check box: [ ] container volume or [X] waste volume 
General physical form (see attached list) ler scrap metals. other (specify)

\section{Chemical form:}

nown.

Waste container type (see attached 1ist)

sert.

Comments (specify number of pertinent question) :

5. Probably also $0, \mathrm{~PB} 3, \mathrm{BXM}, \mathrm{BXW}, \mathrm{RD} 5, \mathrm{BLX}$, and BLM.
2. Details on physical form(particularly confinement related) A variety of metals, such as stainless steel and aluminum.

4. Inner packaging: [ ] plastic bag [X] plastic liner

[ ] metal liner [ ] none [ ] other (specify)

6. Other characteristics of interest: 
- each contaminant, complete at least one line on the following table. If any entries for that contaminant vary by year, fill out additional lines as needed

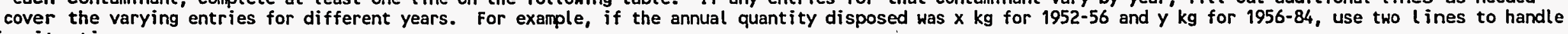
is situation.

\begin{tabular}{|c|c|c|c|c|c|c|c|c|c|c|c|}
\hline Radionucl ide & Physical Form & Chemical Form & $\begin{array}{l}\text { (A)nnual/( } T \text { ) otal } \\
\text { Quantity }\end{array}$ & Unit & $\begin{array}{l}\text { Begin } \\
\text { Year }\end{array}$ & $\begin{array}{l}\text { End } \\
\text { Year }\end{array}$ & $\begin{array}{l}\text { Fore } \\
\text { cast }\end{array}$ & $\begin{array}{l}\text { Samp } \\
\text { les? }\end{array}$ & $\begin{array}{c}\text { Minimum } \\
\text { Value/\#Samp }\end{array}$ & $\begin{array}{c}\text { Maximum } \\
\text { Value/STD }\end{array}$ & $\begin{array}{l}\text { Basis for } \\
\text { Uncertainty }\end{array}$ \\
\hline Cs -137 & Metallic. & Unknown. & T 1.6940000000000 & CI & 1994 & 1994 & N & N & & & \\
\hline Ce -144 & Metallic. & Unknown. & $T .03982000000000$ & CI & 1994 & 1994 & N & N & & & \\
\hline Eu- 154 & Metallic. & Unknown. & T.00002457000000 & $\mathrm{CI}$ & 1994 & 1994 & N & N & & & \\
\hline$E u-155$ & Metallic. & Unknown. & T. .07963000000000 & CI & 1994 & 1994 & N & N & & & \\
\hline$U-234$ & Metallic. & Unknown. & $\mathrm{T} .00001779000000$ & CI & 1994 & 1994 & N & N & & & \\
\hline$U-235$ & Metallic. & Unknown. & T. .00000038120000 & CI & 1994 & 1994 & N & N & & & \\
\hline$v-236$ & Metallic. & Unknown. & T. .000006777700000 & $\mathrm{CI}$ & 1994 & 1994 & N & N & & & \\
\hline$N p-237$ & Metallic. & Unknown. & $T .00001101000000$ & CI & 1994 & 1994 & N & N & & & \\
\hline Pu-238 & Metallic. & Unknown. & $T .00045750000000$ & $\mathrm{CI}$ & 1994 & 1994 & N & N & & & \\
\hline
\end{tabular}

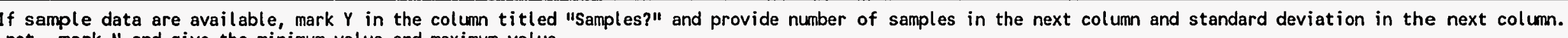
not, mark $N$ and give the minimum value and maximum value.

ditional information or explanations (indicate pertinent contaminant) 
each contaminant, complete at least one line on the following table. If any entries for that contaminant vary by year, fill out additional lines as needed over the varying entries for different years. For example, if the annual quantity disposed was $x \mathrm{~kg}$ for 1952-56 and y $\mathrm{kg}$ for $1956-84$, use two lines to handle situation.

\begin{tabular}{|c|c|c|c|c|c|c|c|c|c|c|c|}
\hline Radionuclide & Physical Form & Chemical Form & $\begin{array}{l}\text { (A)nnual/(T) otal } \\
\text { Quantity }\end{array}$ & Unit & $\begin{array}{l}\text { Begin } \\
\text { Year }\end{array} \mid$ & $\begin{array}{l}\text { End } \\
\text { Year }\end{array}$ & $\begin{array}{l}\text { Fore } \\
\text { cast }\end{array}$ & $\begin{array}{l}\text { Samp } \\
\text { les? }\end{array}$ & $\begin{array}{c}\text { Minimum } \\
\text { Value/\#Samp }\end{array}$ & $\begin{array}{r}\text { Maximum } \\
\text { Value/STD }\end{array}$ & $\begin{array}{l}\text { Basis for } \\
\text { Uncertainty }\end{array}$ \\
\hline Pu-239 & Metallic. & Unknown. & T. .00045750000000 & CI & 1994 & 1994 & N & N & & & \\
\hline Pu-240 & Metallic. & Unknown. & T. .00004829000000 & CI & 1994 & 1994 & N & N & & & \\
\hline Pu-241 & Metall ic. & Unknown. & T.04998000000000 & CI & 1994 & 1994 & N & N & & & \\
\hline$A m-241$ & Metal l ic. & Unknown. & T.00022870000000 & Cl & 1994 & 1994 & N & N & & & \\
\hline $\mathrm{Cm}-242$ & Metal L ic. & Unknown. & T. .00022870000000 & CI & 1994 & 1994 & N & N & & & \\
\hline Cm-244 & Metal L ic. & Unknown. & T. .00021180000000 & Cl & 1994 & 1994 & N & N & & & \\
\hline$H-3$ & Metall ic. & Unknown. & T.69470000000000 & CI & 1995 & 1995 & N & N & & & \\
\hline$c-14$ & Metall ic. & Unknown. & T. .09319000000000 & $\mathrm{CI}$ & 1995 & 1995 & $\mathrm{~N}$ & N & & & \\
\hline Fe-55 & Metall ic. & Unknown. & T 16.100000000000 & CI & 1995 & 1995 & N & $\mathbf{N}$ & & & \\
\hline
\end{tabular}

sample data are available, mark $Y$ in the column titled "Samples?" and provide number of samples in the next column and standard deviation in the next column. 1ot, mark $N$ and give the minimum value and maximum value.

tional information or explanations (indicate pertinent contaminant) 
each contaminant, complete at least one line on the following table. If any entries for that contaminant vary by year, fill out additional lines as needed

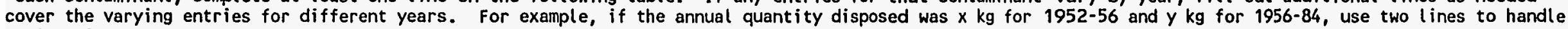
s situation.

\begin{tabular}{|c|c|c|c|c|c|c|c|c|c|c|c|}
\hline Radionuclide & Physical Form & Chemical Form & $\begin{array}{l}\text { (A)nnual/(T)otal } \\
\text { Quantity }\end{array}$ & Unit & $\begin{array}{l}\text { Begin } \\
\text { Year }\end{array}$ & $\begin{array}{l}\text { End } \\
\text { Year }\end{array}$ & $\begin{array}{l}\text { Fore } \\
\text { cast }\end{array}$ & $\begin{array}{l}\text { Samp } \\
\text { les? }\end{array}$ & $\begin{array}{c}\text { Minimum } \\
\text { Value/\#Samp }\end{array}$ & $\begin{array}{r}\text { Maximum } \\
\text { value/STD }\end{array}$ & $\begin{array}{c}\text { Basis for } \\
\text { Uncertainty }\end{array}$ \\
\hline Eu-155 & Metallic. & Unknown. & T. .07963000000000 & CI & 1995 & 1995 & N & N & & & \\
\hline$U-234$ & Metallic. & Unknown. & T. .00001779000000 & CI & 1995 & 1995 & N & N & & & \\
\hline$U-235$ & Metallic. & Unknown. & T. .00000038120000 & $\mathrm{CI}$ & 1995 & 1995 & N & N & & & \\
\hline $\mathrm{U}-236$ & Metallic. & Unknown. & T. .00000677700000 & $\mathrm{Cl}$ & 1995 & 1995 & N & N & & & \\
\hline Np-237 & Metallic. & Unknown. & T. .00001101000000 & CI & 1995 & 1995 & N & N & & & \\
\hline Pu-238 & Metallic. & Unknown. & $T .00045750000000$ & CI & 1995 & 1995 & N & N & & & \\
\hline Pu-239 & Metallic. & Unknown. & $T .00045750000000$ & CI & 1995 & 1995 & N & N & & & \\
\hline Pu-240 & Metallic. & Unknown. & $T .00004829000000$ & CI & 1995 & 1995 & N & N & & & \\
\hline Pu-241 & Metallic. & Unknown. & T. .04998000000000 & CI & 1995 & 1995 & N & N & & & \\
\hline
\end{tabular}

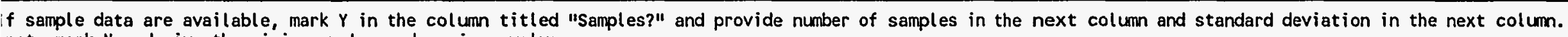
not, mark $N$ and give the minimum value and maximum value.

ditional information or explanations (indicate pertinent contaminant) 
ach contaminant, complete at least one line on the following table. If any entries for that contaminant vary by year, fill out additional lines as needed ver the varying entries for different years. For example, if the annual quantity disposed was $x \mathrm{~kg}$ for $1952-56$ and $y$ kg for $1956-84$, use two lines to handle situation.

\begin{tabular}{|c|c|c|c|c|c|c|c|c|c|c|c|}
\hline adionucl ide & Physical Form & Chemical Form & $\begin{array}{l}\text { (A)nnual } /(T) \text { otal } \\
\text { Quantity }\end{array}$ & Unit & $\begin{array}{l}\text { Begin } \\
\text { Year }\end{array}$ & $\begin{array}{l}\text { End } \\
\text { Year }\end{array}$ & $\begin{array}{l}\text { Fore } \\
\text { cast }\end{array}$ & $\begin{array}{l}\text { Samp } \\
\text { les? }\end{array}$ & $\begin{array}{c}\text { Minimum } \\
\text { Value/\#Samp }\end{array}$ & $\begin{array}{r}\text { Maximum } \\
\text { Value/STo }\end{array}$ & $\begin{array}{l}\text { Basis for } \\
\text { Uncertainty }\end{array}$ \\
\hline-90 & Metallic. & Unknown. & $T .00779400000000$ & $\mathrm{CI}$ & 1996 & 1996 & $N$ & $N$ & & & \\
\hline-99 & Metallic. & Unknown. & T. .00152500000000 & CI & 1996 & 1996 & $N$ & H & & & \\
\hline-129 & Metallic. & Unknown. & T. .00000039645000 & $\mathrm{Cl}$ & 1996 & 1996 & N & N & & & \\
\hline-137 & Metallic. & Unknown. & T 1.6940000000000 & CI & 1996 & 1996 & N & N & & & \\
\hline$=-144$ & Metallic. & Unknown. & T. .03982000000000 & $\mathrm{Cl}$ & 1996 & 1996 & N & N & & & \\
\hline $1-154$ & Metallic. & Unknown. & T. .00002457000000 & CI & 1996 & 1996 & N & N & & & \\
\hline-155 & Metallic. & Unknown. & T. .07963000000000 & CI & 1996 & 1996 & N & N & & & \\
\hline-234 & Metallic. & Unknown. & T. .00001779000000 & CI & 1996 & 1996 & N & N & & & \\
\hline-235 & Metallic. & Unknown. & T.00000038120000 & CI & 1996 & 1996 & $N$ & N & & & \\
\hline
\end{tabular}

sample data are available, mark $Y$ in the column titled "Samples?" and provide number of samples in the next column and standard deviation in the next column. $t$, mark $N$ and give the minimum value and maximum value.

ional information or explanations (indicate pertinent contaminant) 
each contaminant, complete at least one line on the following table. If any entries for that contaminant vary by year, fill out additional lines as needed

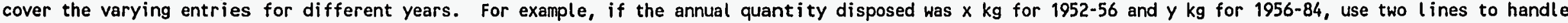
s situation.

\begin{tabular}{|c|c|c|c|c|c|c|c|c|c|c|c|}
\hline Radionuclide & Physical Form & Chemical form & $\begin{array}{l}\text { (A)nnual } /(T) \text { otal } \\
\text { Quantity }\end{array}$ & Unit & $\begin{array}{l}\text { Begin } \\
\text { Year }\end{array}$ & $\begin{array}{l}\text { End } \\
\text { Year }\end{array}$ & $\begin{array}{l}\text { Fore } \\
\text { cast }\end{array}$ & $\begin{array}{l}\text { Samp } \\
\text { les? }\end{array}$ & $\begin{array}{c}\text { Minimum } \\
\text { Value/\#Samp }\end{array}$ & $\begin{array}{r}\text { Maximum } \\
\text { Value/STD }\end{array}$ & $\begin{array}{l}\text { Basis for } \\
\text { Uncertainty }\end{array}$ \\
\hline$H-3$ & Metallic. & Unknown. & T. .69470000000000 & CI & 1997 & 1997 & N & N & & & \\
\hline c- 14 & Metallic. & Unknown. & T. .09319000000000 & $\mathrm{CI}$ & 1997 & 1997 & N & N & & & \\
\hline $\mathrm{Fe}-55$ & Metallic. & Unknown. & T 16.10000000000 & CI & 1997 & 1997 & N & N & & & \\
\hline $\mathrm{Ni}-59$ & Metallic. & Unknown. & $T .00482900000000$ & $\mathrm{CI}$ & 1997 & 1997 & N & N & & & \\
\hline $\mathrm{Ni}-63$ & Metallic. & Unknown. & T 2.7110000000000 & CI & 1997 & 1997 & $N$ & $N$ & & & \\
\hline Co-60 & Metallic. & Unknown. & T 5.6760000000000 & CI & 1997 & 1997 & N & N & & & \\
\hline Sr-90 & Metallic. & Unknown. & $T .00779400000000$ & CI & 1997 & 1997 & N & N & & & \\
\hline Tc-99 & Metallic. & Unknown. & T. .00152500000000 & $\mathrm{CI}$ & 1997 & 1997 & N & N & & & \\
\hline $1-129$ & Metallic. & Unknown. & T. .00000039645000 & CI & 1997 & 1997 & N & $\mathbf{N}$ & & & \\
\hline
\end{tabular}

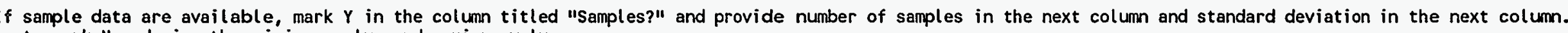
not, mark $N$ and give the minimum value and maximum value.

itional information or explanations (indicate pertinent contaminant) 
- each contaminant, complete at least one line on the following table. If any entries for that contaminant vary by year, fill out additional lines as needed

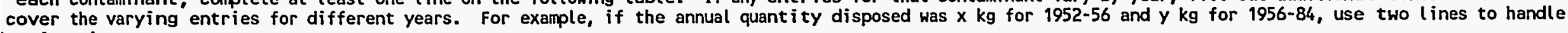
is situation.

\begin{tabular}{|c|c|c|c|c|c|c|c|c|c|c|c|}
\hline Radionuclide & Physical form & Chemical Form & $\begin{array}{l}\text { (A)nnual/(T)otal } \\
\text { Quantity }\end{array}$ & Unit & $\begin{array}{l}\text { Begin } \\
\text { Year }\end{array}$ & $\begin{array}{l}\text { End } \\
\text { Year }\end{array}$ & $\begin{array}{l}\text { Fore } \\
\text { cast }\end{array}$ & $\mid \begin{array}{l}\text { Samp } \\
\text { les? }\end{array}$ & $\begin{array}{c}\text { Minimum } \\
\text { Value/\#Samp }\end{array}$ & $\begin{array}{r}\text { Maximum } \\
\text { Value/STD }\end{array}$ & $\begin{array}{l}\text { Basis for } \\
\text { Uncertainty }\end{array}$ \\
\hline Cs -137 & Metallic. & Unknown. & T 1.6940000000000 & $\mathrm{CI}$ & 1997 & 1997 & N & N & & & \\
\hline Ce-144 & Metallic. & Unknown. & $T .03982000000000$ & $\mathrm{CI}$ & 1997 & 1997 & $\mathbf{N}$ & $\mathbf{N}$ & & & \\
\hline Eu-154 & Metallic. & Unknown. & $T .00002457000000$ & CI & 1997 & 1997 & N & N & & & \\
\hline Eu-155 & Metallic. & Unknown. & $\mathrm{T} .07963000000000$ & CI & 1997 & 1997 & $\mathbf{N}$ & $N$ & & & \\
\hline$U-234$ & Metallic. & Unknown. & T. .00001779000000 & CI & 1997 & 1997 & N & N & & & \\
\hline$U-235$ & Metallic. & Unknown. & $T .00000038120000$ & $\mathrm{Cl}$ & 1997 & 1997 & N & N & & & \\
\hline$U-236$ & Metallic. & Unknown. & $T .00000677700000$ & CI & 1997 & 1997 & N & N & & & \\
\hline$N p-237$ & Metallic. & Unknown. & $T .00001101000000$ & CI & 1997 & 1997 & $N$ & N & & & \\
\hline Pu-238 & Metallic. & Unknown. & $T .00045750000000$ & CI & 1997 & 1997 & N & N & & & \\
\hline
\end{tabular}

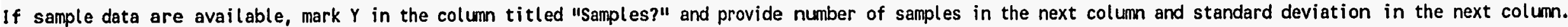
not, mark $N$ and give the minimum value and maximum value.

ditional information or explanations (indicate pertinent contaminant) 
$r$ each contaminant, complete at least one line on the following table. If any entries for that contaminant vary by year, fill out additional lines as needed

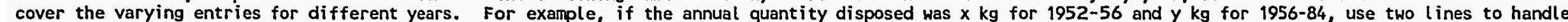
is situation.

\begin{tabular}{|c|c|c|c|c|c|c|c|c|c|c|c|}
\hline Radionuclide & Physical Form & Chemical Form & $\begin{array}{l}\text { (A)nnual/(T)otal } \\
\text { Quantity }\end{array}$ & Unit & $\begin{array}{l}\text { Begin } \\
\text { Year }\end{array}$ & $\begin{array}{l}\text { End } \\
\text { Year }\end{array}$ & $\begin{array}{l}\text { Fore } \\
\text { cast }\end{array}$ & $\begin{array}{l}\text { Samp } \\
\text { les? }\end{array}$ & $\begin{array}{c}\text { Minimum } \\
\text { Value/\#Samp }\end{array}$ & $\begin{array}{r}\text { Maximum } \\
\text { Value/STo }\end{array}$ & $\begin{array}{l}\text { Basis for } \\
\text { Uncertainty }\end{array}$ \\
\hline $\mathrm{Ni}-59$ & Hetallic. & Unknown. & $T .00482900000000$ & CI & 1998 & 1998 & N & N & & & \\
\hline $\mathrm{Ni}-63$ & Hetallic. & Unknown. & T 2.7110000000000 & CI & 1998 & 1998 & $\mathbf{N}$ & N & & & \\
\hline Co-60 & Metallic. & Unknown. & T 5.6760000000000 & $\mathrm{Cl}$ & 1998 & 1998 & N & N & & & \\
\hline $\mathrm{Sr}-90$ & Metallic. & Unknown. & T. .00779400000000 & $\mathrm{Cl}$ & 1998 & 1998 & N & N & & & \\
\hline Tc-99 & Metallic. & Unknown. & T. .00152500000000 & $\mathrm{CI}$ & 1998 & 1998 & $N$ & N & & & \\
\hline $1-129$ & Metallic. & Unknown. & $T .00000039645000$ & CI & 1998 & 1998 & N & N & & & \\
\hline Cs-137 & Metallic. & Unknown. & T 1.6940000000000 & CI & 1998 & 1998 & N & N & & & \\
\hline $\mathrm{Ce}-144$ & Metallic. & Unknown. & $T .03982000000000$ & CI & 1998 & 1998 & N & N & & & \\
\hline Eu-154 & Metallic. & Unknown. & $T .00002457000000$ & CI & 1998 & 1998 & N & N & & & \\
\hline
\end{tabular}

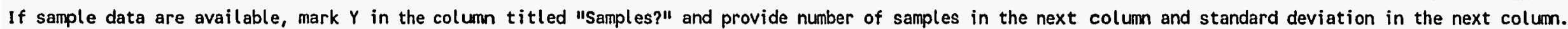
f not, mark $N$ and give the minimum value and maximum value.

dditional information or explanations (indicate pertinent contaminant) 
each contaminant, complete at least one line on the following table. If any entries for that contaminant vary by year, fill out additional lines as needed

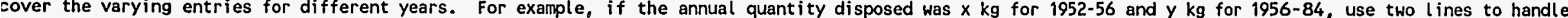
s situation.

\begin{tabular}{|c|c|c|c|c|c|c|c|c|c|c|c|}
\hline Radionuclide & Physical Form & Chemical Form & $\begin{array}{l}\text { (A)nnual/(T)otal } \\
\text { Quantity }\end{array}$ & Unit & $\begin{array}{l}\text { Begin } \\
\text { Year }\end{array}$ & $\begin{array}{l}\text { End } \\
\text { Year }\end{array}$ & $\begin{array}{l}\text { Fore } \\
\text { cast }\end{array}$ & $\begin{array}{l}\text { Samp } \\
\text { les? }\end{array}$ & $\begin{array}{c}\text { Minimum } \\
\text { Value/\#Samp }\end{array}$ & $\begin{array}{r}\text { Maximum } \\
\text { value/STD }\end{array}$ & $\begin{array}{l}\text { Basis for } \\
\text { Uncertainty }\end{array}$ \\
\hline Eu- 955 & Metallic. & Unknown. & T. .07963000000000 & CI & 1998 & 1998 & N & N & & & \\
\hline$U-234$ & Metallic. & Unknown. & T. .00001779000000 & CI & 1998 & 1998 & N & N & & & \\
\hline$U-235$ & Metallic. & Unknown. & T. .00000038120000 & $\mathrm{CI}$ & 1998 & 1998 & N & N & & & \\
\hline$U-236$ & Metallic. & Unknown. & T. .00000677700000 & $\mathrm{Cl}$ & 1998 & 1998 & N & N & & & \\
\hline$N p-237$ & Metallic. & Unknown. & $T .00001101000000$ & $\mathrm{Cl}$ & 1998 & 1998 & N & N & & & \\
\hline Pu-238 & Metallic. & Unknown. & T. .00045750000000 & CI & 1998 & 1998 & N & N & & & \\
\hline Pu-239 & Metallic. & Unknown. & $T .00045750000000$ & $\mathrm{CI}$ & 1998 & 1998 & N & N & & & \\
\hline Pu-240 & Metallic. & Unknown. & $T .00004829000000$ & $\mathrm{CI}$ & 1998 & 1998 & N & N & & & \\
\hline Pu-241 & Metallic. & Unknown. & $T .04998000000000$ & $\mathrm{Cl}$ & 1998 & 1998 & $\mathbf{N}$ & N & & & \\
\hline
\end{tabular}

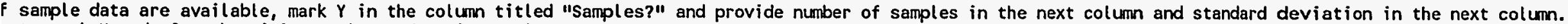
not, mark $N$ and give the minimum value and maximum value.

itional information or explanations (indicate pertinent contaminant) 
- each contaminant, complete at least one line on the following table. If any entries for that contaminant vary by year, fill out additional lines as needed

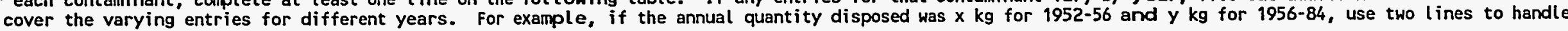
s situation.

\begin{tabular}{|c|c|c|c|c|c|c|c|c|c|c|c|}
\hline Radionucl ide & Physical Form & Chemical Form & $\begin{array}{l}\text { (A)nnual/(T)otal } \\
\text { Quantity }\end{array}$ & Unit & \begin{tabular}{|l|} 
Begin \\
Year
\end{tabular} & $\begin{array}{l}\text { End } \\
\text { Year }\end{array}$ & $\begin{array}{l}\text { Fore } \\
\text { cast }\end{array}$ & $\begin{array}{l}\text { Samp } \\
\text { Les? }\end{array}$ & $\begin{array}{c}\text { Minimum } \\
\text { Value/\#Samp }\end{array}$ & $\begin{array}{r}\text { Maximum } \\
\text { value/STD }\end{array}$ & $\begin{array}{l}\text { Basis for } \\
\text { Uncertainty }\end{array}$ \\
\hline$A m-241$ & Metallic. & Unknown. & $T .00022870000000$ & CI & 1998 & 1998 & N & N & & & \\
\hline $\mathrm{Cm}-242$ & Metallic. & Unknown. & $T .00022870000000$ & CI & 1998 & 1998 & $\mathbf{N}$ & N & & & \\
\hline $\mathrm{Cm}-244$ & Metallic. & Unknown. & $T .00021180000000$ & CI & 1998 & 1998 & $\mathbf{N}$ & N & & & \\
\hline$H-3$ & Metallic. & Unknown. & $T .69470000000000$ & CI & 1999 & 1999 & $\mathbf{N}$ & $N$ & & & \\
\hline C- 14 & Metallic. & Unknown. & $T .09319000000000$ & CI & 1999 & 1999 & $\mathbf{N}$ & N & & & \\
\hline $\mathrm{Fe}-55$ & Metallic. & Unknown. & T 16.100000000000 & CI & 1999 & 1999 & $N$ & N & & & \\
\hline $\mathrm{Ni}-59$ & Metallic. & Unknown. & $T .00482900000000$ & $\mathrm{CI}$ & 1999 & 1999 & N & N & & & \\
\hline $\mathrm{Ni}-63$ & Metallic. & Unknown. & T 2.7110000000000 & $\mathrm{Cl}$ & 1999 & 1999 & $\mathbf{N}$ & N & & & \\
\hline Co- 60 & Metallic. & Unknown. & T 5.6760000000000 & $\mathrm{Cl}$ & 1999 & 1999 & $\mathbf{N}$ & N & & & \\
\hline
\end{tabular}

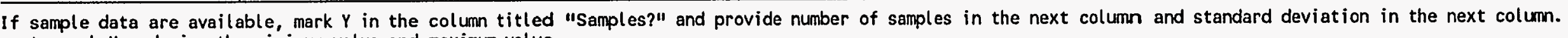
not, mark $N$ and give the minimum value and maximum value.

ditional information or explanations (indicate pertinent contaminant) 
each contaminant, complete at least one line on the following table. If any entries for that contaminant vary by year, fill out additional lines as needed

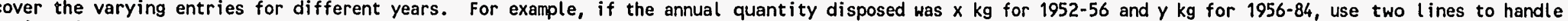
situation.

\begin{tabular}{|c|c|c|c|c|c|c|c|c|c|c|c|}
\hline Radionucl ide & Physical form & Chemical form & $\begin{array}{l}\text { (A)nnual/(T)otal } \\
\text { Quantity }\end{array}$ & Unit & $\begin{array}{l}\text { Begin } \\
\text { Year }\end{array}$ & $\begin{array}{l}\text { End } \\
\text { Year }\end{array}$ & $\begin{array}{l}\text { Fore } \\
\text { cast }\end{array}$ & $\begin{array}{l}\text { Samp } \\
\text { les? }\end{array}$ & $\begin{array}{c}\text { Minimum } \\
\text { Value/\#Samp }\end{array}$ & $\begin{array}{l}\text { Maximum } \\
\text { Value/STD }\end{array}$ & $\begin{array}{l}\text { Basis for } \\
\text { Uncertainty }\end{array}$ \\
\hline $5 r-90$ & Metallic. & Unknown. & T. .00779400000000 & $\mathbf{C I}$ & 1999 & 1999 & N & N & & & \\
\hline TC-99 & Metallic. & Unknown. & $T .00152500000000$ & CI & 1999 & 1999 & N & H & & & \\
\hline$I-129$ & Metallic. & Unknown. & $T .00000039645000$ & CI & 1999 & 1999 & N & N & & & \\
\hline Cs-137 & Metallic. & Unknown. & T 1.6940000000000 & CI & 1999 & 1999 & N & N & & & \\
\hline $\mathrm{Ce}-144$ & Metallic. & Unknown. & $T .03982000000000$ & $\mathrm{CI}$ & 1999 & 1999 & N & N & & & \\
\hline Eu-154 & Metallic. & Unknown. & $T .00002457000000$ & CI & 1999 & 1999 & N & N & & & \\
\hline Eu-155 & Metallic. & Unknown. & $T .07963000000000$ & CI & 1999 & 1999 & N & N & & & \\
\hline$U-234$ & Metallic. & Unknown. & $T .00001779000000$ & CI & 1999 & 1999 & N & N & & & \\
\hline U-235 & Metallic. & Unknown. & $T .00000038120000$ & CI & 1999 & 1999 & N & N & & & \\
\hline
\end{tabular}

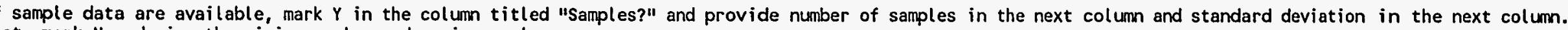
ot, mark $N$ and give the minimum value and maximum value.

tional information or explanations (indicate pertinent contaminant) 
- each contaminant, complete at least one line on the following table. If any entries for that contaminant vary by year, fill out additional lines as needed

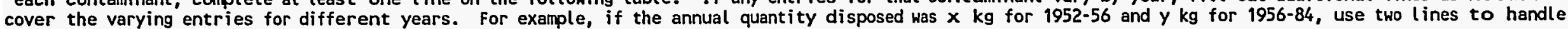
s situation.

\begin{tabular}{|c|c|c|c|c|c|c|c|c|c|c|c|}
\hline Radionuclide & Physical Form & Chemical form & $\begin{array}{l}\text { (A)nnual } /(T) \text { otal } \\
\text { Quantity }\end{array}$ & Unit & $\begin{array}{l}\text { Begin } \\
\text { Year }\end{array}$ & $\begin{array}{l}\text { End } \\
\text { Year }\end{array}$ & $\begin{array}{l}\text { Fore } \\
\text { cast }\end{array}$ & $\begin{array}{l}\text { Samp } \\
\text { Les? }\end{array}$ & \begin{tabular}{|c|} 
Minimum \\
value/\#Samp
\end{tabular} & $\begin{array}{r}\text { Maximum } \\
\text { Value/STD }\end{array}$ & $\begin{array}{l}\text { Basis for } \\
\text { Uncertainty }\end{array}$ \\
\hline $\begin{array}{c}\text { U-236 } \\
.\end{array}$ & Metallic. & Unknown. & T. .00000677700000 & CI & 1999 & 1999 & N & N & & & \\
\hline$N p-237$ & Metallic. & Unknown. & $T .00001101000000$ & CI & 1999 & 1999 & N & N & & & \\
\hline Pu-238 & Metallic. & Unknown. & $T .00045750000000$ & $\mathrm{CI}$ & 1999 & 1999 & N & N & & & \\
\hline Pu-239 & Metallic. & Unknown. & T. .00045750000000 & CI & 1999 & 1999 & N & N & & & \\
\hline Pu-240 & Metallic. & Unknown. & T. .00004829000000 & CI & 1999 & 1999 & N & $N$ & & & \\
\hline $\mathrm{Pu}-241$ & Metallic. & Unknown. & $T .04998000000000$ & CI & 1999 & 1999 & $\mathbf{N}$ & N & & & \\
\hline$A m-241$ & Metall ic. & Unknown. & $T .00022870000000$ & CI & 1999 & 1999 & N & N & & & \\
\hline $\mathrm{Cm}-242$ & Metallic. & Unknown. & $T .00022870000000$ & CI & 1999 & 1999 & N & N & & & \\
\hline $\mathrm{Cm}-244$ & Metallic. & Unknown. & $T .00021180000000$ & CI & 1999 & 1999 & $N$ & N & & & \\
\hline
\end{tabular}

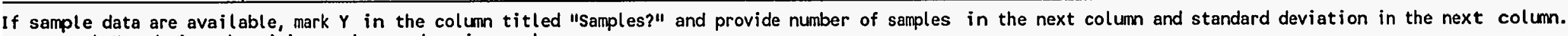
not, mark $N$ and give the minimum value and maximum value.

ditional information or explanations (indicate pertinent contaminant) 
each contaminant, complete at least one line on the following table. If any entries for that contaminant vary by year, fill out additional lines as needed

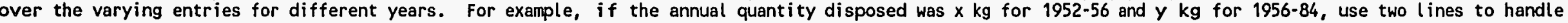
situation.

\begin{tabular}{|c|c|c|c|c|c|c|c|c|c|c|c|}
\hline Radionuclide & Physical Form & Chemical Form & $\begin{array}{l}\text { (A)nnual/(T)otal } \\
\text { Quantity }\end{array}$ & Unit & $\begin{array}{l}\text { Begin } \\
\text { Year }\end{array}$ & $\begin{array}{l}\text { End } \\
\text { Year }\end{array}$ & $\begin{array}{l}\text { Fore } \\
\text { cast }\end{array}$ & $\begin{array}{l}\text { Samp } \\
\text { les? }\end{array}$ & $\begin{array}{c}\text { Minimum } \\
\text { Value/\#Samp }\end{array}$ & $\begin{array}{r}\text { Maximum } \\
\text { Value/STD }\end{array}$ & $\begin{array}{l}\text { Basis for } \\
\text { Uncertainty }\end{array}$ \\
\hline$H-3$ & Metallic. & Unknown. & T.69470000000000 & CI & 2000 & 2000 & $N$ & N & & & \\
\hline$c-14$ & Metallic. & Unknown. & $T .09319000000000$ & $\mathrm{Cl}$ & 2000 & 2000 & $N$ & $\mathbf{N}$ & & & \\
\hline $\mathrm{Fe}-55$ & Metallic. & Unknown. & $T 16.100000000000$ & $\mathrm{CI}$ & 2000 & 2000 & $N$ & $\mathbf{N}$ & & & \\
\hline i -59 & Metallic. & Unknown. & $T .00482900000000$ & CI & 2000 & 2000 & N & $\mathbf{N}$ & & & \\
\hline$N i-63$ & Metallic. & Unknown. & T 2.7110000000000 & CI & 2000 & 2000 & $N$ & $\mathbf{N}$ & & & \\
\hline Co-60 & Metallic. & Unknown. & T 5.6760000000000 & CI & 2000 & 2000 & H & $\mathbf{N}$ & & & \\
\hline $5 r-90$ & Metallic. & Unknown. & $T .00779400000000$ & CI & 2000 & 2000 & N & $\mathbf{N}$ & & & \\
\hline Tc-99 & Metallic. & Unknown. & $T .00152500000000$ & $\mathrm{CI}$ & 2000 & 2000 & N & $\mathbf{N}$ & & & \\
\hline I - 129 & Metallic. & Unknown. & $T .00000039645000$ & $\mathrm{Cl}$ & 2000 & 2000 & $N$ & $\mathbf{N}$ & & & \\
\hline
\end{tabular}

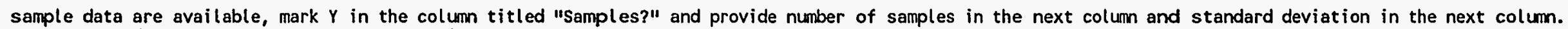

or, mark $N$ and give the minimum value and maximum value.

tional information or explanations (indicate pertinent contaminant) 
each contaminant, complete at least one line on the following table. If any entries for that contaminant vary by year, fill out additional lines as needed over the varying entries for different years. For example, if the annual quantity disposed was $x \mathrm{~kg}$ for $1952-56$ and $y$ kg for $1956-84$, use two lines to handle situation.

\begin{tabular}{|c|c|c|c|c|c|c|c|c|c|c|c|}
\hline Radionuclide & Physical Form & Chemical Form & $\begin{array}{l}\text { (A) nnual /(T)otal } \\
\text { Quantity }\end{array}$ & Unit & $\begin{array}{l}\text { Begin } \\
\text { Year }\end{array}$ & $\begin{array}{l}\text { End } \\
\text { Year }\end{array}$ & $\begin{array}{l}\text { Fore } \\
\text { cast }\end{array}$ & $\begin{array}{l}\text { Samp } \\
\text { les? }\end{array}$ & $\begin{array}{c}\text { Minimum } \\
\text { value/\#Samp }\end{array}$ & $\begin{array}{r}\text { Maximum } \\
\text { Value/STD }\end{array}$ & $\begin{array}{l}\text { Basis for } \\
\text { Uncertainty }\end{array}$ \\
\hline$u-155$ & Metallic. & Unknown. & T. .07963000000000 & CI & 2001 & 2001 & N & N & & & \\
\hline J-234 & Metallic. & Unknown. & $T .00001779000000$ & Cl & 2001 & 2001 & N & N & & & \\
\hline$j-235$ & Metallic. & Unknown. & $T .00000038120000$ & CI & 2001 & 2001 & N & N & & & \\
\hline$J-236$ & Metallic. & Unknown. & T. .00000677700000 & CI & 2001 & 2001 & $\mathrm{~N}$ & N & & & \\
\hline $\mathrm{Np}-237$ & Metallic. & Unknown. & $T .00001101000000$ & CI & 2001 & 2001 & N & N & & & \\
\hline Pu-238 & Metallic. & Unknown. & T. .00045750000000 & CI & 2001 & 2001 & N & N & & & \\
\hline Pu-239 & Metallic. & Unknown. & $T .00045750000000$ & CI & 2001 & 2001 & N & N & & & \\
\hline Pu-240 & Metallic. & Unknown. & $T .00004829000000$ & CI & 2001 & 2001 & N & N & & & \\
\hline Pu-241 & Metallic. & Unknown. & $T .04998000000000$ & CI & 2001 & 2001 & $N$ & $N$ & & & \\
\hline
\end{tabular}

sample data are available, mark $Y$ in the column titled "Samples?" and provide number of samples in the next column and standard deviation in the next column.

1ot, mark $N$ and give the minimum value and maximum value.

tional information or explanations (indicate pertinent contaminant) 
$r$ each contaminant, complete at least one line on the following table. If any entries for that contaminant vary by year, fill out additional lines as needed

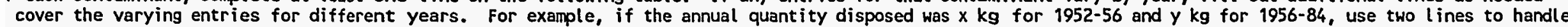
is situation.

\begin{tabular}{|c|c|c|c|c|c|c|c|c|c|c|c|}
\hline Radionucl ide & Physical form & Chemical form & $\begin{array}{l}\text { (A)nnual/(T)otal } \\
\text { Quantity }\end{array}$ & Unit & $\begin{array}{l}\text { Begin } \\
\text { Year }\end{array}$ & $\begin{array}{l}\text { End } \\
\text { Year }\end{array}$ & $\begin{array}{l}\text { Fore } \\
\text { cast }\end{array}$ & $\begin{array}{l}\text { Samp } \\
\text { les? }\end{array}$ & $\begin{array}{c}\text { Minimum } \\
\text { Value/\#Samp }\end{array}$ & $\begin{array}{r}\text { Maximum } \\
\text { Value/STD }\end{array}$ & $\begin{array}{l}\text { Basis for } \\
\text { Uncertainty }\end{array}$ \\
\hline Am-241 & Metallic. & Unknown. & T.00022870000000 & $\mathrm{Cl}$ & 2001 & 2001 & N & N & & & \\
\hline $\mathrm{Cm}-242$ & Metallic. & Unknown. & T.00022870000000 & CI & 2001 & 2001 & $\mathbf{N}$ & N & & & \\
\hline $\mathrm{Cm}-244$ & Metallic. & Unknown. & $T .00021180000000$ & CI & 2001 & 2001 & N & $N$ & & & \\
\hline$H-3$ & Metallic. & Unknown. & $T .69470000000000$ & $\mathrm{CI}$ & 2002 & 2002 & N & N & & & \\
\hline$c-14$ & Metallic. & Unknown. & T. .09319000000000 & $\mathrm{Cl}$ & 2002 & 2002 & N & N & & & \\
\hline $\mathrm{Fe}-55$ & Metallic. & Unknown. & T 16.100000000000 & CI & 2002 & 2002 & N & $N$ & & & \\
\hline $\mathrm{Ni}-59$ & Metallic. & Unknown. & $T .00482900000000$ & $\mathrm{CI}$ & 2002 & 2002 & $N$ & N & & & \\
\hline $\mathrm{Ni}-63$ & Metallic. & Unknown. & T 2.7110000000000 & $\mathrm{CI}$ & 2002 & 2002 & N & N & & & \\
\hline Co-60 & Metallic. & Unknown. & T 5.6760000000000 & $\mathrm{CI}$ & 2002 & 2002 & N & $\mathbf{N}$ & & & \\
\hline
\end{tabular}

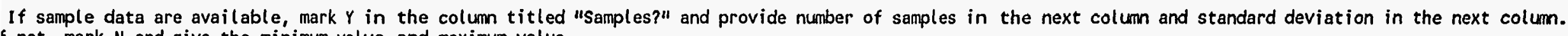
f not, mark $N$ and give the minimum value and maximum value.

dditional information or explanations (indicate pertinent contaminant) 
each contaminant, complete at least one line on the following table. If any entries for that contaminant vary by year, fill out additional lines as needed

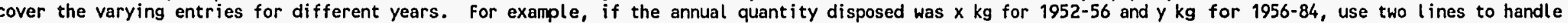
situation.

\begin{tabular}{|c|c|c|c|c|c|c|c|c|c|c|c|}
\hline Radionuclide & Physical Form & Chemical Form & $\begin{array}{l}\text { (A)nnual/(T)otal } \\
\text { Quantity }\end{array}$ & Unit & $\begin{array}{l}\text { Begin } \\
\text { Year }\end{array}$ & $\begin{array}{l}\text { End } \\
\text { Year }\end{array}$ & $\begin{array}{l}\text { Fore } \\
\text { cast }\end{array}$ & $\begin{array}{l}\text { Samp } \\
\text { les? }\end{array}$ & $\begin{array}{c}\text { Minimum } \\
\text { value/\#Samp }\end{array}$ & $\begin{array}{r}\text { Maximum } \\
\text { Value/STD }\end{array}$ & $\begin{array}{l}\text { Basis for } \\
\text { Uncertainty }\end{array}$ \\
\hline Sr-90 & Metallic. & Unknown. & T. .00779400000000 & CI & 2002 & 2002 & N & N & & & \\
\hline TC- 99 & Metallic. & Unknown. & T. .00152500000000 & $\mathrm{CI}$ & 2002 & 2002 & N & N & & & \\
\hline $1-129$ & Metallic. & Unknown. & T. .00000039645000 & $\mathrm{Cl}$ & 2002 & 2002 & N & N & & & \\
\hline Cs- 137 & Metallic. & Unknown. & T 1.6940000000000 & $\mathrm{CI}$ & 2002 & 2002 & $N$ & N & & & \\
\hline $\mathrm{Ce}-144$ & Metallic. & Unknown. & T. .03982000000000 & CI & 2002 & 2002 & N & N & & & \\
\hline Eu-154 & Metallic. & Unknown. & $T .00002457000000$ & $\mathrm{CI}$ & 2002 & 2002 & N & N & & & \\
\hline Eu-155 & Metallic. & Unknown. & $T .07963000000000$ & CI & 2002 & 2002 & N & N & & & \\
\hline$U-234$ & Metallic. & Unknown. & $\tau .00001779000000$ & CI & 2002 & 2002 & N & N & & & \\
\hline U-235 & Metallic. & Unknown. & $T .00000038120000$ & CI & 2002 & 2002 & N & N & & & \\
\hline
\end{tabular}

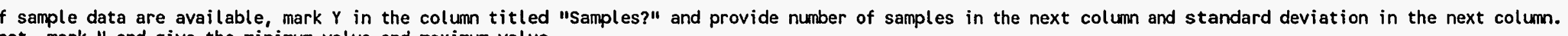
not, mark $N$ and give the minimum value and maximum value.

itional information or explanations (indicate pertinent contaminant) 
- each contaminant, complete at least one line on the following table. If any entries for that contaminant vary by year, fill out additional lines as needed

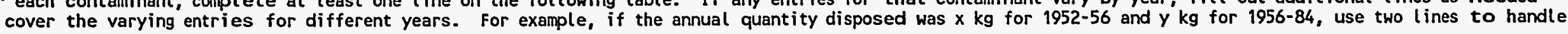
is situation.

\begin{tabular}{|c|c|c|c|c|c|c|c|c|c|c|c|}
\hline Radionucl ide & Physical Form & Chemical Form & $\begin{array}{l}\text { (A)nnual/(T)otal } \\
\text { Quantity }\end{array}$ & Unit & $\begin{array}{l}\text { Begin } \\
\text { Year }\end{array}$ & $\begin{array}{l}\text { End } \\
\text { Year }\end{array}$ & $\begin{array}{l}\text { Fore } \\
\text { cast }\end{array}$ & $\begin{array}{l}\text { Samp } \\
\text { les? }\end{array}$ & $\begin{array}{c}\text { Minimum } \\
\text { Value/\#Samp }\end{array}$ & $\begin{array}{r}\text { Maximum } \\
\text { Value/STD }\end{array}$ & $\begin{array}{l}\text { Basis for } \\
\text { Uncertainty }\end{array}$ \\
\hline$U-236$ & Metallic. & Unknown. & T. .00000677700000 & CI & 2002 & 2002 & N & N & & & \\
\hline$N p-237$ & Metallic. & Unknown. & T.00001101000000 & CI & 2002 & 2002 & $\mathbf{N}$ & N & & & \\
\hline$P u-238$ & Metallic. & Unknown. & T. .00045750000000 & CI & 2002 & 2002 & N & N & & & \\
\hline Pu-239 & Metallic. & Unknown. & T. .00045750000000 & $\mathrm{CI}$ & 2002 & 2002 & $\mathbf{N}$ & $\mathbf{N}$ & & & \\
\hline Pu-240 & Metallic. & Unknown. & T. .00004829000000 & $\mathrm{CI}$ & 2002 & 2002 & N & $\mathbf{N}$ & & & \\
\hline Pu-241 & Metallic. & Unknown. & T. .04998000000000 & $\mathrm{CI}$ & 2002 & 2002 & N & N & & & \\
\hline$A m-241$ & Metallic. & Unknown. & T. .00022870000000 & $\mathrm{Cl}$ & 2002 & 2002 & N & N & & & \\
\hline Cm-242 & Metallic. & Unknown. & $T .00022870000000$ & CI & 2002 & 2002 & $N$ & N & & & \\
\hline $\mathrm{Cm}-244$ & Metallic. & Unknown. & T. .00021180000000 & CI & 2002 & 2002 & N & $N$ & & & \\
\hline
\end{tabular}

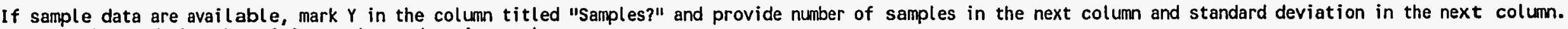
not, mark $H$ and give the minimum value and maximum value.

ditional information or explanations (indicate pertinent contaminant) 
each contaminant, complete at least one line on the following table. If any entries for that contaminant vary by year, fill out additional lines as needed

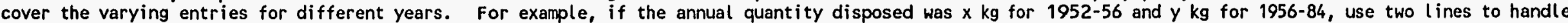
s situation.

\begin{tabular}{|c|c|c|c|c|c|c|c|c|c|c|c|}
\hline Radionuclide & Physical Form & Chemical form & $\begin{array}{l}\text { (A)nnual /(T ) otal } \\
\text { Quantity }\end{array}$ & Unit & $\begin{array}{l}\text { Begin } \\
\text { Year }\end{array}$ & $\begin{array}{l}\text { End } \\
\text { Year }\end{array}$ & $\begin{array}{l}\text { Fore } \\
\text { cast }\end{array}$ & $\begin{array}{l}\text { Samp } \\
\text { les? }\end{array}$ & $\begin{array}{c}\text { Minimum } \\
\text { Value/\#Samp }\end{array}$ & $\begin{array}{r}\text { Maximum } \\
\text { Value/STD }\end{array}$ & $\begin{array}{l}\text { Bas is for } \\
\text { Uncertainty }\end{array}$ \\
\hline$H-3$ & Metallic. & Unknown. & $T .69470000000000$ & $\mathrm{Cl}$ & 2003 & 2003 & N & $\mathbf{N}$ & & & \\
\hline$C-14$ & Metallic. & Unknown. & $T .09319000000000$ & $\mathrm{CI}$ & 2003 & 2003 & N & N & & & \\
\hline $\mathrm{Fe}-55$ & Metallic. & Unknown. & T 16.100000000000 & $\mathrm{Cl}$ & 2003 & 2003 & $\mathbf{N}$ & N & & & \\
\hline $\mathrm{Ni}-59$ & Metallic. & Unknown. & $T .00482900000000$ & CI & 2003 & 2003 & N & N & & & \\
\hline $\mathrm{Ni}-63$ & Metallic. & Unknown. & T 2.7110000000000 & CI & 2003 & 2003 & $\mathbf{N}$ & $\mathrm{N}$ & & & \\
\hline Co-60 & Metallic. & Unknown. & T 5.6760000000000 & $\mathrm{CI}$ & 2003 & 2003 & N & N & & & \\
\hline$S r-90$ & Metallic. & Unknown. & T.00779400000000 & CI & 2003 & 2003 & $\mathbf{N}$ & N & & & \\
\hline Tc-99 & Metallic. & Unknown. & $T .00152500000000$ & CI & 2003 & 2003 & $\mathrm{~N}$ & $\mathbf{N}$ & & & \\
\hline $1-129$ & Metallic. & Unknown. & $T .00000039645000$ & CI & 2003 & 2003 & $\mathbf{N}$ & $\mathbf{N}$ & & & \\
\hline
\end{tabular}

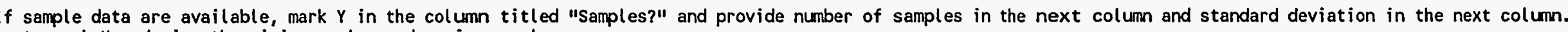
not, mark $N$ and give the minimum value and maximum value.

itional information or explanations (indicate pertinent contaminant) 
or each contaminant, complete at least one line on the following table. If any entries for that contaminant vary by year, fill out additional lines as needed

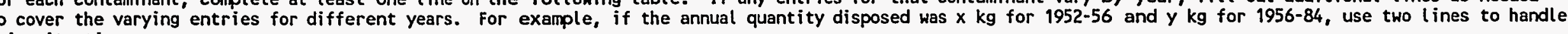
is situation.

\begin{tabular}{|c|c|c|c|c|c|c|c|c|c|c|c|}
\hline Radionucl ide & Physical Form & Chemical form & $\begin{array}{l}\text { (A)nnual/(T)otal } \\
\text { Quantity }\end{array}$ & Unit & $\begin{array}{l}\text { Begin } \\
\text { Year }\end{array}$ & $\begin{array}{l}\text { End } \\
\text { Year }\end{array}$ & $\begin{array}{l}\text { Fore } \\
\text { cast }\end{array}$ & $\begin{array}{l}\text { Samp } \\
\text { les? }\end{array}$ & $\begin{array}{c}\text { Minimum } \\
\text { Value/\#Samp }\end{array}$ & $\begin{array}{c}\text { Maximum } \\
\text { Value/sto }\end{array}$ & $\begin{array}{l}\text { Basis for } \\
\text { Uncertainty }\end{array}$ \\
\hline Cs-137 & Metallic. & Unknown. & T 1.6940000000000 & CI & 2003 & 2003 & N & N & & & \\
\hline Ce-144 & Metallic. & Unknown. & T. .03982000000000 & CI & 2003 & 2003 & N & N & & & \\
\hline Eu-154 & Metallic. & Unknown. & $T .00002457000000$ & CI & 2003 & 2003 & N & N & & & \\
\hline Eu-155 & Metallic. & Unknown. & $T .07963000000000$ & CI & 2003 & 2003 & $\mathbf{N}$ & N & & & \\
\hline$U-234$ & Metallic. & Unknown. & T. .00001779000000 & CI & 2003 & 2003 & $\mathbf{N}$ & N & & & \\
\hline U-235 & Metallic. & Unknown. & T. .00000038120000 & CI & 2003 & 2003 & N & N & & & \\
\hline$U-236$ & Metallic. & Unknown. & T. .00000677700000 & CI & 2003 & 2003 & N & N & & & \\
\hline$N p-237$ & Metallic. & Unknown. & T.00001101000000 & CI & 2003 & 2003 & $N$ & N & & & \\
\hline Pu-238 & Metallic. & Unknown. & $T .00045750000000$ & CI & 2003 & 2003 & $\mathbf{N}$ & $\mathbf{N}$ & & & \\
\hline
\end{tabular}

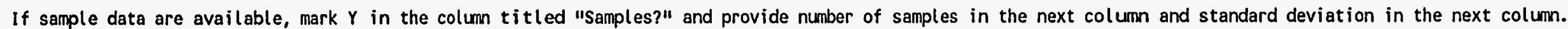
$f$ not, mark $N$ and give the minimum value and maximum value.

dditional information or explanations (indicate pertinent contaminant) 
Type of source of information: check box)

X] RWMIS [ ] other database

] sample analysis data

operating records [ ] interview

] expert judgment [ ] reports

generator forecasts

other

- Do the estimates of contaminant

uantities in Part $C$ and $D$ represent:

$\mathrm{X}$ ] best estimate

] worst case

] other

- Do the data conflict with RWMIS? (Historical or Present Data only) ] no

$\mathrm{x}]$ yes

- Major unknowns in inventories of :ontaminants:

inidentified beta gamma (U-B-G) were inknown. The $C i$ content was unknown.
2. Details concerning source (names, report no., dates, etc.)

4. If other than best estimate, explain why:

6. If yes, explain why:

A portion of or all of the measurements were assumed to be taken using a G-M detector which only measures gamma emitters; beta emitters have been added to the RWMIS activity based on scaling factors. Therefore, the total activity will be greater than that in RWMIS.

8. Key assumptions used to deal with the unknowns: U-B-G were identified based on EPRI NP-5077 for PWRs. PWR was chosen because it is similar to ATR, the reactor at TRA. The radionuclides chosen were based on a combination of the following: cross section, half lives and fission products. The preparer averaged the $\mathrm{Ci}$ content over 1984-1993, then multiplied that number by 10 to reflect the number of projected years. 
DATA INPUT FOR HISTORICAL DATA TASK FOR RWMC SUBSURFACE DISPOSAL AREA

\section{RT A - GENERAL INFORMATION PDT - 298}

Preparer: Amaro, C.

Generator: TRA

cea or contractor - use code from attached list)

Number of waste stream from this facility:

$27 \mathrm{P}$

Type of radioactive waste (check box):

TRU or suspect TRU

LLW

non-radioactive

Actual years disposed of at SDA:

arting year 1994 Ending year 2003
2. Date prepared: $07 / 16 / 94$

4. Particular facility: 603

(building number - use code from attached list)

6. Waste stream:

Non-compactible waste, such as glass, metal, etc.

9. Waste stream volume:

Amount $\quad 250.0000$ Units Cubic meters.

Check box: [ ] annual or [X] total over all years

Check box: [ ] container volume or [X] waste volume

Comments (specify number of pertinent question):

4. Also 670 and probably several other buildings. 
- General physical form (see attached list) ther scrap metals. $\mathrm{X}$ ] other (specify) lass.

\section{- 'Chemical form:} nknown.

- Waste container type (see attached list) ooden box.

- Comments (specify number of pertinent question): 5. Probably also BLM and other containers.
2. Details on physical form(particularly confinement related) This stream is a combination of non-compactible items, such as glass and metal.

4. Inner packaging: [ ] plastic bag [X] plastic liner [ ] metal liner [ ] none [ ] other (specify)
6. Other characteristics of interest: 


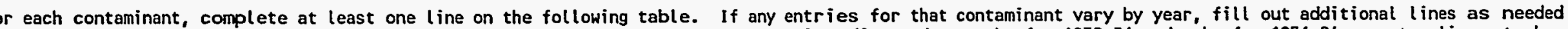

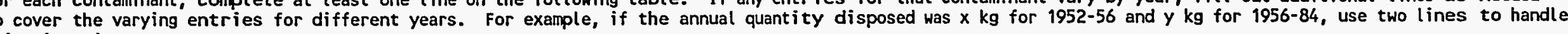
is situation.

\begin{tabular}{|c|c|c|c|c|c|c|c|c|c|c|c|}
\hline Radionuclide & Physical Form & Chemical Form & $\begin{array}{l}\text { (A)nnual / ( T)otal } \\
\text { Quantity }\end{array}$ & Unit & $\begin{array}{l}\text { Begin } \\
\text { Year }\end{array}$ & $\begin{array}{l}\text { End } \\
\text { Year }\end{array}$ & $\begin{array}{l}\text { Fore } \\
\text { cast }\end{array}$ & $\begin{array}{l}\text { Samp } \\
\text { les? }\end{array}$ & $\begin{array}{c}\text { Minimum } \\
\text { Value/\#Samp }\end{array}$ & $\begin{array}{c}\text { Maximum } \\
\text { Value/STD }\end{array}$ & $\begin{array}{l}\text { Basis for } \\
\text { Uncertainty }\end{array}$ \\
\hline$H-3$ & Unknown. & Unknown. & T 2.0460000000000 & CI & 1994 & 1994 & $Y$ & N & & & \\
\hline$C-14$ & Unknown. & Unknown. & T. .2744000000000 & $\mathrm{Cl}$ & 1994 & 1994 & $Y$ & N & & & \\
\hline Fe-55 & Unknown. & Unknown. & T 47.400000000000 & CI & 1994 & 1994 & $Y$ & N & & & \\
\hline $\mathrm{Ni}-59$ & Unknown. & Unknown. & $T .01422000000000$ & CI & 1994 & 1994 & Y & N & & & \\
\hline $\mathrm{Ni}-63$ & Unknown. & Unknown. & T 7.9840000000000 & CI & 1994 & 1994 & $Y$ & N & & & \\
\hline Co- 60 & Unknown. & Unknown. & T 16.720000000000 & CI & 1994 & 1994 & Y & N & & & \\
\hline $\mathrm{Sr}-90$ & Unknown. & Unknown. & T. .02295000000000 & CI & 1994 & 1994 & $Y$ & N & & & \\
\hline TC-99 & Unknown. & Unknown. & T.00449100000000 & CI & 1994 & 1994 & $Y$ & N & & & \\
\hline I-129 & Unknown. & Unknown. & T. .00000116730000 & CI & 1994 & 1994 & $Y$ & N & & & \\
\hline
\end{tabular}

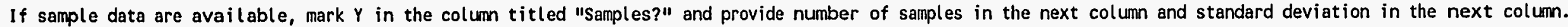

f not, mark $N$ and give the minimum value and maximum value.

dditional information or explanations (indicate pertinent contaminant) 
each contaminant, complete at least one line on the following table. If any entries for that contaminant vary by year, fill out additional lines as needed

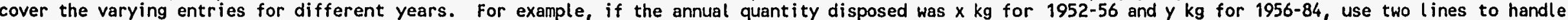
s situation.

\begin{tabular}{|c|c|c|c|c|c|c|c|c|c|c|c|}
\hline Radionuclide & Physical form & Chemical form & $\begin{array}{l}\text { (A)nnual/(T)otal } \\
\text { Quantity }\end{array}$ & Unit & $\begin{array}{l}\text { Begin } \\
\text { Year }\end{array}$ & $\begin{array}{l}\text { End } \\
\text { Year }\end{array}$ & $\begin{array}{l}\text { Fore } \\
\text { cast }\end{array}$ & $\begin{array}{l}\text { Samp } \\
\text { les? }\end{array}$ & $\begin{array}{c}\text { Minimum } \\
\text { Value/\#Samp }\end{array}$ & $\begin{array}{r}\text { Maximum } \\
\text { Value/STD }\end{array}$ & $\begin{array}{l}\text { Basis for } \\
\text { Uncertainty }\end{array}$ \\
\hline Cs- 137 & Unknown. & Unknown. & Y 4.9900000000000 & $\mathrm{CI}$ & 1994 & 1994 & $Y$ & N & & & \\
\hline Ce- 144 & Unknown. & Unknown. & $T .11730000000000$ & CI & 1994 & 1994 & $Y$ & N & & & \\
\hline Eu-154 & Unknown. & Unknown. & $T .00007235000000$ & CI & 1994 & 1994 & $Y$ & $N$ & & & \\
\hline Eu-155 & Unknown. & Unknown. & $T .23450000000000$ & CI & 1994 & 1994 & $Y$ & N & & & \\
\hline U-234 & Unknown. & Unknown. & $T .00005239000000$ & $\mathrm{CI}$ & 1994 & 1994 & $Y$ & $N$ & & & \\
\hline$U-235$ & Unknown. & Unknown. & T. .00000112300000 & CI & 1994 & 1994 & $Y$ & $\mathbf{N}$ & & & \\
\hline$U-236$ & Unknown. & Unknown. & T. .00001996000000 & $\mathrm{Cl}$ & 1994 & 1994 & $\mathbf{Y}$ & N & & & \\
\hline Np-237 & Unknown. & Unknown. & $T .00003243000000$ & CI & 1994 & 1994 & $\mathbf{Y}$ & N & & & \\
\hline Pu-238 & Unknown. & Unknown. & T. .00134700000000 & CI & 1994 & 1994 & $=Y$ & $\mathbf{N}$ & & & \\
\hline
\end{tabular}

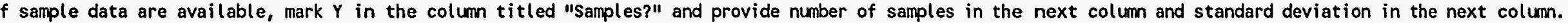
not, mark $N$ and give the minimum value and maximum value.

itional information or explanations (indicate pertinent contaminant) 


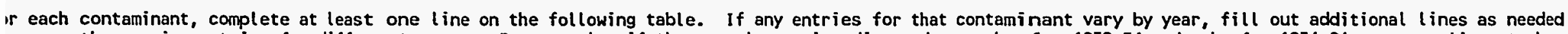

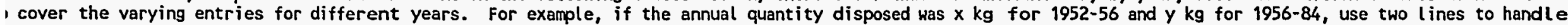
is situation.

\begin{tabular}{|c|c|c|c|c|c|c|c|c|c|c|c|}
\hline Radionuclide & Physical Form & Chemical form & $\begin{array}{l}\text { (A)nnual/(T)otal } \\
\text { Quant ity }\end{array}$ & Unit & $\begin{array}{l}\text { Begin } \\
\text { Year }\end{array}$ & $\begin{array}{l}\text { End } \\
\text { Year }\end{array}$ & $\begin{array}{l}\text { Fore } \\
\text { cast }\end{array}$ & $\begin{array}{l}\text { Samp } \\
\text { les? }\end{array}$ & $\begin{array}{c}\text { Minimum } \\
\text { Value/\#Samp }\end{array}$ & $\begin{array}{l}\text { Maximum } \\
\text { value/STD }\end{array}$ & $\begin{array}{l}\text { Basis for } \\
\text { Uncertainty }\end{array}$ \\
\hline Pu-239 & Unknown. & Unknown. & T.00134700000000 & CI & 1994 & 1994 & Y & N & & & \\
\hline Pu-240 & Unknown. & Unknown. & T. .00014220000000 & CI & 1994 & 1994 & $Y$ & N & & & \\
\hline$P u-241$ & Unknown. & Unknown. & T. 14720000000000 & CI & 1994 & 1994 & Y & $N$ & & & \\
\hline$A m-241$ & Unknown. & Unknown. & T. .00067360000000 & CI & 1994 & 1994 & Y & N & & & \\
\hline $\mathrm{Cm}-242$ & Unknown. & Unknown. & T. .00067360000000 & CI & 1994 & 1994 & $Y$ & N & & & \\
\hline $\mathrm{Cm}-244$ & Unknown. & Unknown. & T. .00062370000000 & CI & 1994 & 1994 & $Y$ & N & & & \\
\hline $\mathrm{H}-3$ & Unknown. & Unknown. & T 2.0460000000000 & CI & 1995 & 1995 & $Y$ & N & & & \\
\hline$C-14$ & Unknown. & Unknown. & T.27440000000000 & CI & 1995 & 1995 & $Y$ & N & & & \\
\hline $\mathrm{Fe}-55$ & Unknown. & Unknown. & T 47.400000000000 & CI & 1995 & 1995 & Y & N & & & \\
\hline
\end{tabular}

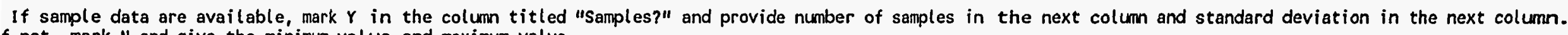
f not, mark $N$ and give the minimum value and maximum value.

dditional information or explanations (indicate pertinent contaminant) 
each contaminant, complete at least one line on the following table. If any entries for that contaminant vary by year, fill out additional lines as needed

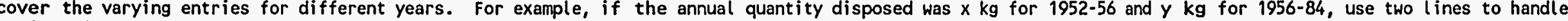
situation.

\begin{tabular}{|c|c|c|c|c|c|c|c|c|c|c|c|}
\hline Radionucl ide & Physical Form & Chemical Form & $\begin{array}{l}\text { (A)nnual/( } T \text { ) otal } \\
\text { Quantity }\end{array}$ & Unit & $\begin{array}{l}\text { Begin } \\
\text { Year }\end{array}$ & $\begin{array}{l}\text { End } \\
\text { Year }\end{array}$ & $\begin{array}{l}\text { Fore } \\
\text { cast }\end{array}$ & $\begin{array}{l}\text { Samp } \\
\text { les? }\end{array}$ & $\begin{array}{c}\text { Minimum } \\
\text { Value/\#Samp }\end{array}$ & $\begin{array}{r}\text { Maximum } \\
\text { value/STD }\end{array}$ & $\begin{array}{l}\text { Basis for } \\
\text { Uncertainty }\end{array}$ \\
\hline $\mathrm{Ni}-59$ & Unknown. & Unknown. & T. .01422000000000 & $\mathrm{CI}$ & 1995 & 1995 & $Y$ & N & & & \\
\hline $\mathrm{Ni}-63$ & Unknown. & Unknown. & T 7.9840000000000 & $\mathrm{CI}$ & 1995 & 1995 & $Y$ & $\mathbf{N}$ & & & \\
\hline Co- 60 & Unknown. & Unknown. & T 16.720000000000 & CI & 1995 & 1995 & $\gamma$ & N & & & \\
\hline$S r-90$ & Unknown. & Unknown. & T.02295000000000 & $\mathrm{CI}$ & 1995 & 1995 & $Y$ & N & & & \\
\hline Tc-99 & Unknown. & Unknown. & T. .00449100000000 & CI & 1995 & 1995 & $Y$ & N & & & \\
\hline $1-129$ & Unknown. & Unknown. & T. .00000116730000 & CI & 1995 & 1995 & $\gamma$ & N & & & \\
\hline Cs-137 & Unknown. & Unknown. & T 4.9900000000000 & $\mathrm{Cl}$ & 1995 & 1995 & $Y$ & N & & & \\
\hline $\mathrm{Ce}-144$ & Unknown. & Unknown. & T.11730000000000 & CI & 1995 & 1995 & $Y$ & $\mathbf{N}$ & & & \\
\hline Eu- 154 & Unknown. & Unknown. & T. .00007235000000 & CI & 1995 & 1995 & $Y$ & N & & & \\
\hline
\end{tabular}

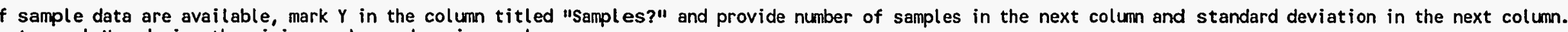
not, mark $N$ and give the minimum value and maximum value.

itional information or explanations (indicate pertinent contaminant) 


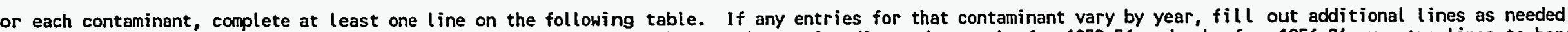

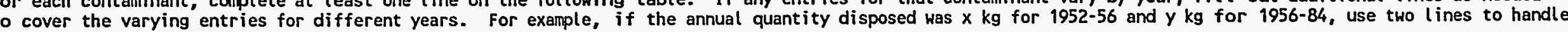
his situation.

\begin{tabular}{|c|c|c|c|c|c|c|c|c|c|c|c|}
\hline Radionuclide & Physical form & Chemical Form & $\begin{array}{l}\text { (A)nnual/(T)otal } \\
\text { Quantity }\end{array}$ & Unit & $\begin{array}{l}\text { Begin } \\
\text { Year }\end{array}$ & $\begin{array}{l}\text { End } \\
\text { Year }\end{array}$ & $\begin{array}{l}\text { Fore } \\
\text { cast }\end{array}$ & $\begin{array}{l}\text { Samp } \\
\text { les? }\end{array}$ & $\begin{array}{c}\text { Minimum } \\
\text { Value/\#Samp }\end{array}$ & $\begin{array}{r}\text { Maximum } \\
\text { Value/STD }\end{array}$ & $\begin{array}{l}\text { Basis for } \\
\text { Uncertainty }\end{array}$ \\
\hline Eu-155 & Unknown. & Unknown. & T. .23450000000000 & CI & 1995 & 1995 & $\gamma$ & N & & & \\
\hline$U-234$ & Unknown. & Unknown. & T. .00005239000000 & CI & 1995 & 1995 & Y & N & & & \\
\hline$U-235$ & Unknown. & Unknown. & T. .00000112300000 & CI & 1995 & 1995 & $Y$ & N & & & \\
\hline$U-236$ & Unknown. & Unknown. & $T .00001996000000$ & CI & 1995 & 1995 & $Y$ & N & & & \\
\hline Np-237 & Unknown. & Unknown. & T. .00003243000000 & CI & 1995 & 1995 & $Y$ & N & & & \\
\hline Pu-238 & Unknown. & Unknown. & T. .00134700000000 & $\mathrm{CI}$ & 1995 & 1995 & $Y$ & N & & & \\
\hline Pu-239 & Unknown. & Unknown. & T. .00134700000000 & $\mathrm{Cl}$ & 1995 & 1995 & $Y$ & N & & & \\
\hline Pu-240 & Unknown. & Unknown. & $T .00014220000000$ & CI & 1995 & 1995 & Y & N & & & \\
\hline Pu-241 & Unknown. & Unknown. & T. .14720000000000 & CI & 1995 & 1995 & $\mathbf{Y}$ & N & & & \\
\hline
\end{tabular}

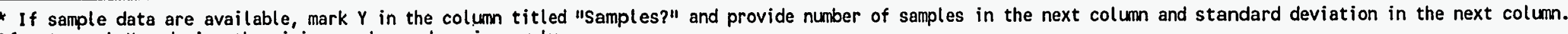
If not, mark $N$ and give the minimum value and maximum value.

Additional information or explanations (indicate pertinent contaminant) 
each contaminant, complete at least one line on the following table. If any entries for that contaminant vary by year, fill out additional lines as needed

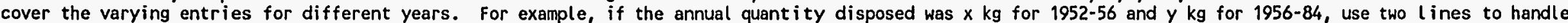
s situation.

\begin{tabular}{|c|c|c|c|c|c|c|c|c|c|c|c|}
\hline Radionucl ide & Physical Form & Chemical form & $\begin{array}{l}\text { (A)nnual/(T)otal } \\
\text { Quantity }\end{array}$ & Unit & $\begin{array}{l}\text { Begin } \\
\text { Year }\end{array}$ & $\begin{array}{l}\text { End } \\
\text { Year }\end{array}$ & $\begin{array}{l}\text { Fore } \\
\text { cast }\end{array}$ & $\begin{array}{l}\text { Samp } \\
\text { les? }\end{array}$ & $\begin{array}{c}\text { Minimum } \\
\text { Value/\#Samp }\end{array}$ & $\begin{array}{r}\text { Maximum } \\
\text { Value/STD }\end{array}$ & $\begin{array}{l}\text { Basis for } \\
\text { Uncertainty }\end{array}$ \\
\hline$A m-241$ & Unknown. & Unknown. & $T .00067360000000$ & CI & 1995 & 1995 & $Y$ & N & & & \\
\hline $\mathrm{Cm}-242$ & Unknown. & Unknown. & T.00067360000000 & $\mathrm{Cl}$ & 1995 & 1995 & $\mathbf{Y}$ & $\mathbf{N}$ & & & \\
\hline $\mathrm{Cm}-244$ & Unknown. & Unknown. & T. .00062370000000 & $\mathrm{Cl}$ & 1995 & 1995 & $Y$ & $N$ & & & \\
\hline $\mathrm{H}-3$ & Unknown. & Unknown. & T 2.046000000000 & $\mathrm{Cl}$ & 1996 & 1996 & $Y$ & N & & & \\
\hline$c-14$ & Unknown. & Unknown. & T. .27440000000000 & $\mathrm{Cl}$ & 1996 & 1996 & $\mathbf{Y}$ & N & & & \\
\hline $\mathrm{Fe}-55$ & Unknown. & Unknown. & T 47.400000000000 & $\mathrm{CI}$ & 1996 & 1996 & $Y$ & $N$ & & & \\
\hline Ni-59 & Unknown. & Unknown. & $T .01422000000000$ & CI & 1996 & 1996 & $Y$ & $N$ & & & \\
\hline $\mathrm{Ni}-63$ & Unknown. & Unknown. & T 7.9840000000000 & CI & 1996 & 1996 & $y$ & N & & & \\
\hline $\mathrm{Co}-60$ & Unknown. & Unknown. & T 16.720000000000 & Cl & 1996 & 1996 & $Y$ & N & & & \\
\hline
\end{tabular}

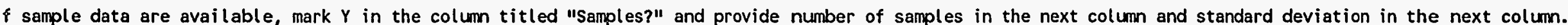
not, mark $N$ and give the minimum value and maximum value.

itional information or explanations (indicate pertinent contaminant) 
each contaminant, complete at least one line on the following table. If any entries for that contaminant vary by year, fill out additional lines as needed

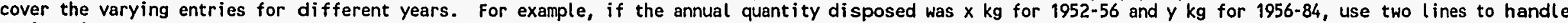
s situation.

\begin{tabular}{|c|c|c|c|c|c|c|c|c|c|c|c|}
\hline Radionuclide & Physical form & Chemical form & $\begin{array}{l}\text { (A) nnual / ( T )otal } \\
\text { Quant i ty }\end{array}$ & Unit & $\begin{array}{l}\text { Begin } \\
\text { Year }\end{array}$ & $\begin{array}{l}\text { End } \\
\text { Year }\end{array}$ & $\begin{array}{l}\text { Fore } \\
\text { cast }\end{array}$ & $\begin{array}{l}\text { Samp } \\
\text { les? }\end{array}$ & $\begin{array}{c}\text { Minimum } \\
\text { Value/\#Samp }\end{array}$ & $\begin{array}{r}\text { Maximum } \\
\text { Value/STD }\end{array}$ & $\begin{array}{l}\text { Basis for } \\
\text { Uncertainty }\end{array}$ \\
\hline$U-236$ & Unknown. & Unknown. & T.00001996000000 & $\mathrm{Cl}$ & 1996 & 1996 & $Y$ & N & & & \\
\hline Np-237 & Unknown. & Unknown. & T. .00003243000000 & CI & 1996 & 1996 & $Y$ & N & & & \\
\hline Pu-238 & Unknown. & Unknown. & T. .00134700000000 & $\mathrm{Cl}$ & 1996 & 1996 & $Y$ & N & & & \\
\hline Pu-239 & Unknown. & Unknown. & T. .00134700000000 & CI & 1996 & 1996 & $Y$ & N & & & \\
\hline Pu-240 & Unknown. & Unknown. & T. .00014220000000 & CI & 1996 & 1996 & $Y$ & N & & & \\
\hline Pu-241 & Unknown. & Unknown. & T. .14720000000000 & $\mathrm{CI}$ & 1996 & 1996 & $Y$ & N & & & \\
\hline$A m-241$ & Unknown. & Unknown. & T. .00067360000000 & CI & 1996 & 1996 & $\mathbf{Y}$ & N & & & \\
\hline $\mathrm{Cm}-242$ & Unknown. & Unknown. & T. .00067360000000 & CI & 1996 & 1996 & $Y$ & $N$ & & & \\
\hline $\mathrm{Cm}-244$ & Unknown. & Unknown. & T. .00062370000000 & CI & 1996 & 1996 & $Y$ & N & & & \\
\hline
\end{tabular}

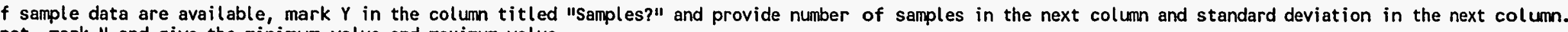
not, mark $N$ and give the minimum value and maximum value.

itional information or explanations (indicate pertinent contaminant) 


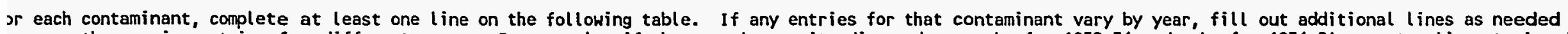

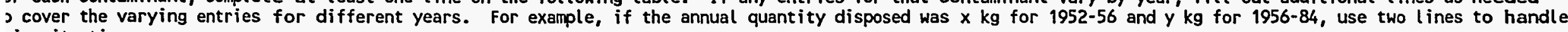
iis situation.

\begin{tabular}{|c|c|c|c|c|c|c|c|c|c|c|c|}
\hline Radionuclide & Physical Form & Chemical Form & $\begin{array}{l}\text { (A)nnual/(T)otal } \\
\text { Quantity }\end{array}$ & Unit & $\begin{array}{l}\text { Begin } \\
\text { Year }\end{array}$ & $\begin{array}{l}\text { End } \\
\text { Year }\end{array}$ & $\begin{array}{l}\text { Fore } \\
\text { cast }\end{array}$ & $\begin{array}{l}\text { Samp } \\
\text { les? }\end{array}$ & $\begin{array}{c}\text { Minimum } \\
\text { Value/\#Samp }\end{array}$ & $\begin{array}{r}\text { Maximum } \\
\text { value/STD }\end{array}$ & $\begin{array}{l}\text { Basis for } \\
\text { Uncertainty }\end{array}$ \\
\hline$H-3$ & Unknown. & Unknown. & T 2.0460000000000 & CI & 1997 & 1997 & $Y$ & N & & & \\
\hline$c-14$ & Unknown. & Unknown. & T.27440000000000 & CI & 1997 & 1997 & $Y$ & N & & & \\
\hline Fe-55 & Unknown. & Unknown. & T 47.400000000000 & CI & 1997 & 1997 & $Y$ & N & & & \\
\hline $\mathrm{Ni}-59$ & Unknown. & Unknown. & T. .01422000000000 & CI & 1997 & 1997 & Y & N & & & \\
\hline $\mathrm{Ni}-63$ & Unknown. & Unknown. & T 7.9840000000000 & CI & 1997 & 1997 & $Y$ & N & & & \\
\hline Co-60 & Unknown. & Unknown. & T 16.720000000000 & $\mathrm{Cl}$ & 1997 & 1997 & $Y$ & N & & & \\
\hline$S r-90$ & Unknown. & Unknown. & T.02295000000000 & $\mathrm{CI}$ & 1997 & 1997 & $Y$ & N & & & \\
\hline Tc-99 & Unknown. & Unknown. & T.00449100000000 & CI & 1997 & 1997 & $Y$ & $N$ & & & \\
\hline$I-129$ & Unknown. & Unknown. & T. .00000116730000 & CI & 1997 & 1997 & $Y$ & N & & & \\
\hline
\end{tabular}

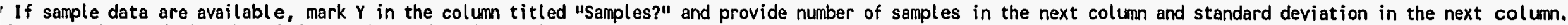
f not, mark $N$ and give the minimum value and maximum value.

idditional information or explanations (indicate pertinent contaminant) 
- each contaminant, complete at least one line on the following table. If any entries for that contaminant vary by year, fill out additional lines as needed

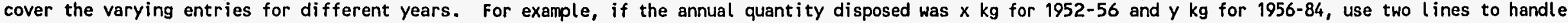
is situation.

\begin{tabular}{|c|c|c|c|c|c|c|c|c|c|c|c|}
\hline Radionucl ide & Physical Form & Chemical Form & $\begin{array}{l}\text { (A)nnual/(T)otal } \\
\text { Quantity }\end{array}$ & Unit & $\begin{array}{l}\text { Begin } \\
\text { Year }\end{array}$ & $\begin{array}{l}\text { End } \\
\text { Year }\end{array}$ & $\begin{array}{l}\text { Fore } \\
\text { cast }\end{array}$ & $\begin{array}{l}\text { Samp } \\
\text { les? }\end{array}$ & $\begin{array}{c}\text { Minimum } \\
\text { Value/\#Samp }\end{array}$ & $\begin{array}{r}\text { Maximum } \\
\text { Value/STD }\end{array}$ & $\begin{array}{l}\text { Basis for } \\
\text { Uncertainty }\end{array}$ \\
\hline Cs-137 & Unknown. & Unknown. & T 4.9900000000000 & CI & 1997 & 1997 & $Y$ & $N$ & & & \\
\hline $\mathrm{Ce}-144$ & Unknown. & Unknown. & T.11730000000000 & $\mathrm{CI}$ & 1997 & 1997 & $Y$ & N & & & \\
\hline Eu- 154 & Unknown. & Unknown. & T. .00007235000000 & CI & 1997 & 1997 & $Y$ & N & & & \\
\hline Eu- 155 & Unknown. & Unknown. & $T .23450000000000$ & $\mathrm{CI}$ & 1997 & 1997 & $Y$ & $N$ & & & \\
\hline$U-234$ & Unknown. & Unknown. & T. .00005239000000 & $\mathrm{Cl}$ & 1997 & 1997 & $\gamma$ & N & & & \\
\hline$U-235$ & Unknown. & Unknown. & T. .00000112300000 & CI & 1997 & 1997 & $\gamma$ & N & & & \\
\hline$U-236$ & Unknown. & Unknown. & $T .00001996000000$ & $\mathrm{CI}$ & 1997 & 1997 & $Y$ & N & & & \\
\hline Np-237 & Unknown. & Unknown. & $T .00003243000000$ & $\mathrm{Cl}$ & 1997 & 1997 & $Y$ & N & & & \\
\hline Pu-238 & Unknown. & Unknown. & $T .00134700000000$ & $\mathrm{CI}$ & 1997 & 1997 & $\mathbf{Y}$ & N & & & \\
\hline
\end{tabular}

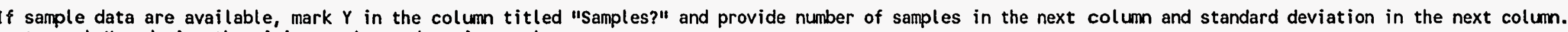
not, mark $N$ and give the minimum value and maximum value.

ditional information or explanations (indicate pertinent contaminant) 
$r$ each contaminant, complete at least one line on the following table. If any entries for that contaminant vary by year, fill out additional lines as needed

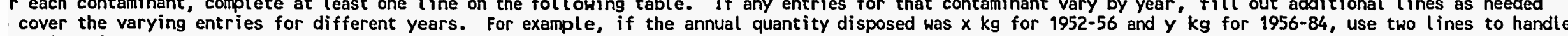
is situation.

\begin{tabular}{|c|c|c|c|c|c|c|c|c|c|c|c|}
\hline Radionucl ide & Physical Form & Chemical Form & $\begin{array}{l}\text { (A)nnual/(T)otal } \\
\text { Quantity }\end{array}$ & Unit & $\begin{array}{l}\text { Begin } \\
\text { Year }\end{array}$ & $\begin{array}{l}\text { End } \\
\text { Year }\end{array}$ & $\begin{array}{l}\text { Fore } \\
\text { cast }\end{array}$ & $\begin{array}{l}\text { Samp } \\
\text { les? }\end{array}$ & $\begin{array}{c}\text { Minimum } \\
\text { Value/\#Samp }\end{array}$ & $\begin{array}{r}\text { Maximum } \\
\text { Value/STD }\end{array}$ & $\begin{array}{l}\text { Basis for } \\
\text { Uncertainty }\end{array}$ \\
\hline Eu-155 & Unknown. & Unknown. & T.23450000000000 & CI & 1998 & 1998 & $Y$ & N & & & \\
\hline$U-234$ & Unknown. & Unknown. & T. .00005239000000 & CI & 1998 & 1998 & $\gamma$ & $\mathbf{N}$ & & & \\
\hline$U-235$ & Unknown. & Unknown. & $T .00000112300000$ & CI & 1998 & 1998 & $\gamma$ & N & & & \\
\hline$U-236$ & Unknown. & Unknown. & T. .00001996000000 & $\mathrm{CI}$ & 1998 & 1998 & $\gamma$ & N & & & \\
\hline Np-237 & Unknown. & Unknown. & $T .00003243000000$ & $\mathrm{Cl}$ & 1998 & 1998 & $Y$ & N & & & \\
\hline Pu-238 & Unknown. & Unknown. & T. .00134700000000 & CI & 1998 & 1998 & $Y$ & N & & & \\
\hline Pu-239 & Unknown. & Unknown. & $T .00134700000000$ & CI & 1998 & 1998 & $Y$ & $N$ & & & \\
\hline$P u-240$ & Unknown. & Unknown. & $T .00014220000000$ & CI & 1998 & 1998 & $Y$ & $\boldsymbol{N}$ & & & \\
\hline Pu-241 & Unknown. & Unknown. & $T .14720000000000$ & $\mathrm{CI}$ & 1998 & 1998 & $Y$ & $\mathbf{N}$ & & & \\
\hline
\end{tabular}

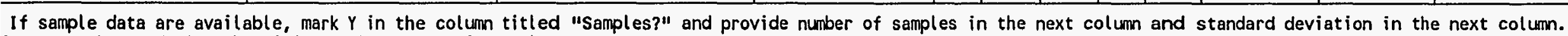
$f$ not, mark $N$ and give the minimum value and maximum value.

dditional information or explanations (indicate pertinent contaminant) 
each contaminant, complete at least one line on the following table. If any entries for that contaminant vary by year, fill out additional lines as needed

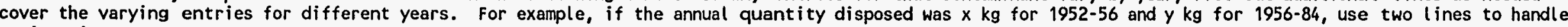
s situation.

\begin{tabular}{|c|c|c|c|c|c|c|c|c|c|c|c|}
\hline Radionucl ide & Physical Form & Chemical form & $\begin{array}{l}\text { (A)nnual /(T)otal } \\
\text { Quantity }\end{array}$ & Unit & $\begin{array}{l}\text { Begin } \\
\text { Year }\end{array}$ & $\begin{array}{l}\text { End } \\
\text { Year }\end{array}$ & $\begin{array}{l}\text { Fore } \\
\text { cast }\end{array}$ & $\begin{array}{l}\text { Samp } \\
\text { les? }\end{array}$ & $\begin{array}{c}\text { Minimum } \\
\text { Value/\#Samp }\end{array}$ & $\begin{array}{r}\text { Maximum } \\
\text { Value/STD }\end{array}$ & $\begin{array}{l}\text { Basis for } \\
\text { Uncertainty }\end{array}$ \\
\hline Am-241 & Unknown. & Unknown. & T. .00067360000000 & CI & 1998 & 1998 & $Y$ & N & & & \\
\hline $\mathrm{Cm}-242$ & Unknown. & Unknown. & T. .00067360000000 & CI & 1998 & 1998 & $Y$ & $N$ & & & \\
\hline $\mathrm{Cm}-244$ & Unknown. & Unknown. & T. .00062370000000 & CI & 1998 & 1998 & $Y$ & $N$ & & & \\
\hline$H-3$ & Unknown. & Unknown. & T 2.0460000000000 & CI & 1999 & 1999 & $\gamma$ & $N$ & & & \\
\hline$C-14$ & Unknown. & Unknown. & $T .27440000000000$ & $\mathrm{CI}$ & 1999 & 1999 & $\gamma$ & N & & & \\
\hline $\mathrm{Fe}-55$ & Unknown. & Unknown. & T 47.400000000000 & CI & 1999 & 1999 & $Y$ & N & & & \\
\hline $\mathrm{Ni}-59$ & Unknown. & Unknown. & T. .01422000000000 & $\mathrm{Cl}$ & 1999 & 1999 & $Y$ & $N$ & & & \\
\hline $\mathrm{Ni}-63$ & Unknown. & Unknown. & T 7.9840000000000 & CI & 1999 & 1999 & Y & N & & & \\
\hline Co-60 & Unknown. & Unknown. & T 16.720000000000 & CI & 1999 & 1999 & $Y$ & $N$ & & & \\
\hline
\end{tabular}

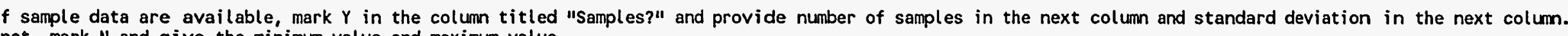
not, mark $N$ and give the minimum value and maximum value.

itional information or explanations (indicate pertinent contaminant) 
each contaminant, complete at least one line on the following table. If any entries for that contaminant vary by year, fill out additional lines as needed

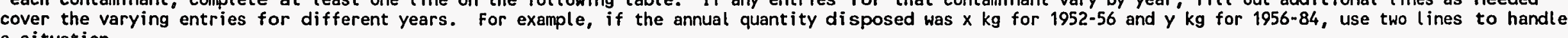
s situation.

\begin{tabular}{|c|c|c|c|c|c|c|c|c|c|c|c|}
\hline Radionucl ide & Physical Form & Chemical Form & $\begin{array}{l}\text { (A)nnual / }(T \text { ) otal } \\
\text { Quant i ty }\end{array}$ & Unit & $\begin{array}{l}\text { Begin } \\
\text { Year }\end{array}$ & $\begin{array}{l}\text { End } \\
\text { Year }\end{array}$ & $\begin{array}{l}\text { Fore } \\
\text { cast }\end{array}$ & $\begin{array}{l}\text { Samp } \\
\text { les? }\end{array}$ & $\begin{array}{c}\text { Minimum } \\
\text { Value/\#Samp }\end{array}$ & $\begin{array}{c}\text { Maximum } \\
\text { value/STD }\end{array}$ & $\begin{array}{l}\text { Basis for } \\
\text { Uncertainty }\end{array}$ \\
\hline$U-236$ & Unknown. & Unknown. & T. .00001996000000 & CI & 1999 & 1999 & Y & N & & & \\
\hline Np-237 & Unknown. & Unknown. & $T .00003243000000$ & $\mathrm{Cl}$ & 1999 & 1999 & $Y$ & N & & & \\
\hline Pu-238 & Unknown. & Unknown. & T.00134700000000 & $\mathrm{CI}$ & 1999 & 1999 & $Y$ & N & & & \\
\hline Pu-239 & Unknown. & Unknown. & $T .00134700000000$ & $\mathrm{Cl}$ & 1999 & 1999 & $\gamma$ & N & & & \\
\hline Pu-240 & Unknown. & Unknown. & $T .00014220000000$ & CI & 1999 & 1999 & $Y$ & N & & & \\
\hline Pu-24? & Unknown. & Unknown. & T.14720000000000 & CI & 1999 & 1999 & $Y$ & $N$ & & & \\
\hline$A m-241$ & Unknown. & Unknown. & $T .00067360000000$ & $\mathrm{Cl}$ & 1999 & 1999 & $Y$ & N & & & \\
\hline $\mathrm{Cm}-242$ & Unknown. & Unknown. & T.00067360000000 & CI & 1999 & 1999 & $Y$ & $\mathbf{N}$ & & & \\
\hline $\mathrm{Cm}-244$ & Unknown. & Unknown. & T. .00062370000000 & CI & 1999 & 1999 & $\mathbf{Y}$ & $\mathbf{N}$ & & & \\
\hline
\end{tabular}

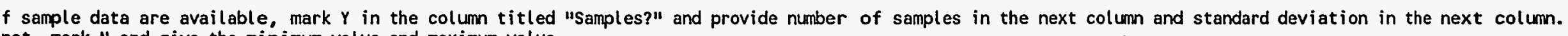
not, mark $N$ and give the minimum value and maximum value.

itional information or explanations (indicate pertinent contaminant) 
r each contaminant, complete at least one line on the following table. If any entries for that contaminant vary by year, fill out additional lines as needed

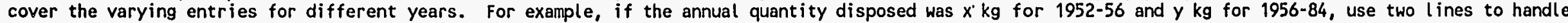
is situation.

\begin{tabular}{|c|c|c|c|c|c|c|c|c|c|c|c|}
\hline Radionuclide & Physical form & Chemical form & $\begin{array}{l}\text { (A)nnual / ( T )otal } \\
\text { Quantity }\end{array}$ & Unit & $\begin{array}{l}\text { Begin } \\
\text { Year }\end{array}$ & $\begin{array}{l}\text { End } \\
\text { Year }\end{array}$ & 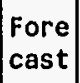 & $\begin{array}{l}\text { Samp } \\
\text { les? }\end{array}$ & $\begin{array}{c}\text { Minimum } \\
\text { Value/\#Samp }\end{array}$ & $\begin{array}{r}\text { Maximum } \\
\text { Value/STD }\end{array}$ & $\begin{array}{l}\text { Basis for } \\
\text { Uncertainty }\end{array}$ \\
\hline Cs- 137 & Unknown. & Unknown. & T 4.9900000000000 & CI & 2000 & 2000 & $\mathbf{Y}$ & N & & & \\
\hline Ce-144 & Unknown. & Unknown. & T. .11730000000000 & CI & 2000 & 2000 & 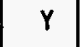 & $N$ & & & \\
\hline Eu-154 & Unknown. & Unknown. & $T .00007235000000$ & CI & 2000 & 2000 & Y & N & & & \\
\hline EU-155 & Unknown. & Unknown. & T. .23450000000000 & $\mathrm{Cl}$ & 2000 & 2000 & $Y$ & N & & & \\
\hline$U-234$ & Unknown. & Unknown. & T. .00005239000000 & $\mathrm{CI}$ & 2000 & 2000 & $Y$ & $N$ & & & \\
\hline$U-235$ & Unknown. & Unknown. & $T .00000112300000$ & CI & 2000 & 2000 & $\mathbf{Y}$ & N & & & \\
\hline $\mathbf{U}-236$ & Unknown. & Unknown. & T.00001996000000 & CI & 2000 & 2000 & $Y$ & N & & & \\
\hline$N p-237$ & Unknown. & Unknown. & T. .00003243000000 & CI & 2000 & 2000 & $Y$ & N & & & \\
\hline Pu-238 & Unknown. & Unknown. & T.00134700000000 & $\mathrm{Cl}$ & 2000 & 2000 & Y & M & & & \\
\hline
\end{tabular}

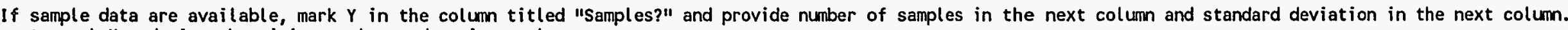
not, mark $N$ and give the minimum value and maximum value.

ditional information or explanations (indicate pertinent contaminant) 
each contaminant, complete at least one line on the following table. If any entries for that contaminant vary by year, fill out additional lines as needed over the varying entries for different years. For example, if the annual quantity disposed was $x \mathrm{~kg}$ for 1952-56 and y $\mathrm{kg}$ for $1956-84$, use two lines to handle situation.

\begin{tabular}{|c|c|c|c|c|c|c|c|c|c|c|c|}
\hline Radionuclide & Physical Form & Chemical Form & $\begin{array}{l}\text { (A)nnual/(T) otal } \\
\text { Quant ity }\end{array}$ & Unit & $\begin{array}{l}\text { Begin } \\
\text { Year }\end{array}$ & $\begin{array}{l}\text { End } \\
\text { Year }\end{array}$ & $\begin{array}{l}\text { Fore } \\
\text { cast }\end{array}$ & $\begin{array}{l}\text { Samp } \\
\text { les? }\end{array}$ & $\begin{array}{c}\text { Minimum } \\
\text { Value/\#Samp }\end{array}$ & $\begin{array}{r}\text { Maximum } \\
\text { value/STD }\end{array}$ & $\begin{array}{l}\text { Basis for } \\
\text { Uncertainty }\end{array}$ \\
\hline$i-59$ & Unknown. & Unknown. & T. .01422000000000 & CI & 2001 & 2001 & $Y$ & N & & & \\
\hline$i-63$ & Unknown. & Unknown. & T 7.9840000000000 & Cl & 2001 & 2001 & $Y$ & N & & & \\
\hline $0-60$ & Unknown. & Unknown. & T 16.720000000000 & $\mathrm{CI}$ & 2001 & 2001 & Y & N & & & \\
\hline$r-90$ & Unknown. & Unknown. & $T .02295000000000$ & CI & 2001 & 2001 & Y & $\mathbf{N}$ & & & \\
\hline c-99 & Unknown. & Unknown. & T.00449100000000 & CI & 2001 & 2001 & $Y$ & N & & & \\
\hline $1-129$ & Unknown. & Unknown. & $T .00000116730000$ & CI & 2001 & 2001 & $Y$ & N & & & \\
\hline Cs -137 & Unknown. & Unknown. & T 4.9900000000000 & CI & 2001 & 2001 & $Y$ & N & & & \\
\hline $\mathrm{Ce}-144$ & Unknown. & Unknown. & T. .11730000000000 & $\mathrm{CI}$ & 2001 & 2001 & $\mathbf{Y}$ & N & & & \\
\hline Eu-154 & Unknown. & Unknown. & T. .00007235000000 & $\mathrm{CI}$ & 2001 & 2001 & $Y$ & $N$ & & & \\
\hline
\end{tabular}

sample data are available, mark $Y$ in the column titled "Samples?" and provide number of samples in the next column and standard deviation in the next column. ot, mark $N$ and give the minimum value and maximum value.

tional information or explanations (indicate pertinent contaminant) 


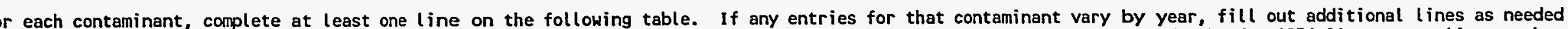

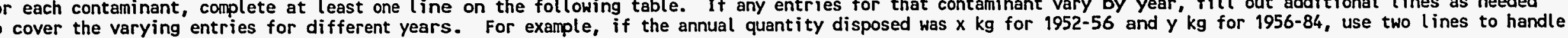
is situation.

\begin{tabular}{|c|c|c|c|c|c|c|c|c|c|c|c|}
\hline Radionucl ide & Physical Form & Chemical Form & $\begin{array}{l}\text { (A)nnual/(T)otal } \\
\text { Quantity }\end{array}$ & Unit & $\begin{array}{l}\text { Begin } \\
\text { Year }\end{array}$ & $\begin{array}{l}\text { End } \\
\text { Year }\end{array}$ & $\begin{array}{l}\text { Fore } \\
\text { cast }\end{array}$ & $\begin{array}{l}\text { Samp } \\
\text { les? }\end{array}$ & $\begin{array}{c}\text { Minimum } \\
\text { Value/\#Samp }\end{array}$ & $\begin{array}{c}\text { Maximum } \\
\text { Value/STD }\end{array}$ & $\begin{array}{l}\text { Basis for } \\
\text { Uncertainty }\end{array}$ \\
\hline Eu-155 & Unknown. & Unknown. & T. .23450000000000 & CI & 2001 & 2001 & $\gamma$ & N & & & \\
\hline$U-234$ & Unknown. & Unknown. & T. .00005239000000 & CI & 2001 & 2001 & $\mathbf{Y}$ & N & & & \\
\hline U-235 & Unknown. & Unknown. & $T .00000112300000$ & $\mathrm{CI}$ & 2001 & 2001 & $\mathbf{Y}$ & N & & & \\
\hline$U-236$ & Unknown. & Unknown. & T. .00001996000000 & CI & 2001 & 2001 & $Y$ & N & & & \\
\hline Np-237 & Unknown. & Unknown. & $T .00003243000000$ & CI & 2001 & 2001 & $Y$ & N & & & \\
\hline Pu-238 & Unknown. & Unknown. & T. .00134700000000 & CI & 2001 & 2001 & $Y$ & N & & & \\
\hline Pu-239 & Unknown. & Unknown. & $r .00134700000000$ & CI & 2001 & 2001 & $\mathbf{Y}$ & N & & & \\
\hline Pu-240 & Unknown. & Unknown. & $T .00014220000000$ & Cl & 2001 & 2001 & $Y$ & N & & & \\
\hline Pu-241 & Unknown. & Unknown. & $T .14720000000000$ & $\mathrm{CI}$ & 2001 & 2001 & $\mathbf{Y}$ & N & & & \\
\hline
\end{tabular}

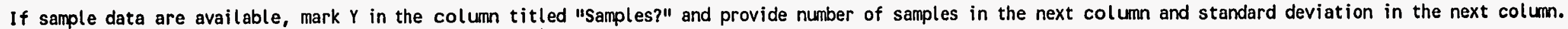
f not, mark $N$ and give the minimum value and maximum value.

dditional information or explanations (indicate pertinent contaminant) 
each contaminant, complete at least one line on the following table. If any entries for that contaminant vary by year, fill out additional lines as needed

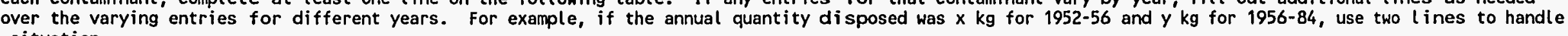
situation.

\begin{tabular}{|c|c|c|c|c|c|c|c|c|c|c|c|}
\hline Radionucl ide & Physical Form & Chemical Form & $\begin{array}{l}\text { (A)nnual / ( T ) otal } \\
\text { Quant i ty }\end{array}$ & Unit & $\begin{array}{l}\text { Begin } \\
\text { Year }\end{array}$ & $\begin{array}{l}\text { End } \\
\text { Year }\end{array}$ & $\begin{array}{l}\text { Fore } \\
\text { cast }\end{array}$ & $\begin{array}{l}\text { Samp } \\
\text { les? }\end{array}$ & $\begin{array}{c}\text { Minimum } \\
\text { Value/\#Samp }\end{array}$ & $\begin{array}{l}\text { Maximum } \\
\text { Value/STD }\end{array}$ & $\begin{array}{l}\text { Basis for } \\
\text { Uncertainty }\end{array}$ \\
\hline$U-236$ & Unknown. & Unknown. & T.00001996000000 & CI & 2002 & 2002 & $Y$ & N & & & \\
\hline Np-237 & Unknown. & Unknown. & $T .00003243000000$ & $\mathrm{CI}$ & 2002 & 2002 & $Y$ & N & & & \\
\hline Pu-238 & Unknown. & Unknown. & T. .00134700000000 & CI & 2002 & 2002 & $y$ & $\mathbf{N}$ & & & \\
\hline Pu-239 & Unknown. & Unknown. & $T .00134700000000$ & CI & 2002 & 2002 & $Y$ & N & & & \\
\hline Pu-240 & Unknown. & Unknown. & T. .00014220000000 & CI & 2002 & 2002 & $Y$ & N & & & \\
\hline Pu-241 & Unknown. & Unknown. & T. .14720000000000 & $\mathrm{CI}$ & 2002 & 2002 & $Y$ & $N$ & & & \\
\hline$A m-241$ & Unknown. & Unknown. & $T .00067360000000$ & $\mathrm{CI}$ & 2002 & 2002 & $Y$ & $N$ & & & \\
\hline $\mathrm{Cm}-242$ & Unknown. & Unknown. & $T .00067360000000$ & CI & 2002 & 2002 & $Y$ & N & & & \\
\hline $\mathrm{Cm}-244$ & Unknown. & Unknown. & T. .00062370000000 & $\mathrm{CI}$ & 2002 & 2002 & $\mathbf{Y}$ & $N$ & & & \\
\hline
\end{tabular}

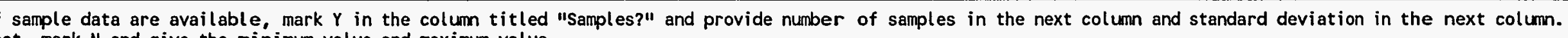
ot, mark $N$ and give the minimum value and maximum value.

tional information or explanations (indicate pertinent contaminant) 


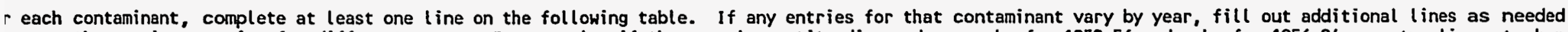

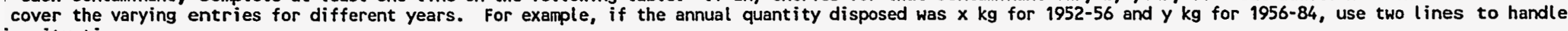
is situation.

\begin{tabular}{|c|c|c|c|c|c|c|c|c|c|c|c|}
\hline Radionuclide & Physical Form & Chemical Form & $\begin{array}{l}\text { (A)nnual /(T)otal } \\
\text { Quantity }\end{array}$ & Unit & $\begin{array}{l}\text { Begin } \\
\text { Year }\end{array}$ & $\begin{array}{l}\text { End } \\
\text { Year }\end{array}$ & $\begin{array}{l}\text { Fore } \\
\text { cast }\end{array}$ & $\begin{array}{l}\text { Samp } \\
\text { les? }\end{array}$ & $\begin{array}{c}\text { Minimum } \\
\text { Value/\#Samp }\end{array}$ & $\begin{array}{r}\text { Maximum } \\
\text { Value/STD }\end{array}$ & $\begin{array}{l}\text { 8asis for } \\
\text { Uncertainty }\end{array}$ \\
\hline$H-3$ & Unknown. & Unknown. & T 2.0460000000000 & Cl & 2003 & 2003 & $Y$ & N & & & \\
\hline$c-14$ & Unknown. & Unknown. & T.27440000000000 & Cl & 2003 & 2003 & $\mathbf{Y}$ & N & & & \\
\hline $\mathrm{Fe}-55$ & Unknown. & Unknown. & T 47.400000000000 & CI & 2003 & 2003 & $\mathbf{Y}$ & N & & & \\
\hline $\mathrm{Ni}-59$ & Unknown. & Unknown. & T. .01422000000000 & CI & 2003 & 2003 & $Y$ & N & & & \\
\hline $\mathrm{Ni}-63$ & Unknown. & Unknown. & T 7.9840000000000 & CI & 2003 & 2003 & $Y$ & N & & & \\
\hline Co- 60 & Unknown. & Unknown. & T 16.720000000000 & CI & 2003 & 2003 & $\mathbf{Y}$ & $\mathbf{N}$ & & & \\
\hline $\mathrm{Sr}-90$ & Unknown. & Unknown. & $T .02295000000000$ & $\mathrm{Cl}$ & 2003 & 2003 & $Y$ & N & & & \\
\hline Tc-99 & Unknown. & Unknown. & $T .00449100000000$ & CI & 2003 & 2003 & $\mathbf{Y}$ & N & & & \\
\hline$I-129$ & Unknown. & Unknown. & T.00000116730000 & CI & 2003 & 2003 & $\mathbf{Y}$ & $\mathbf{N}$ & & & \\
\hline
\end{tabular}

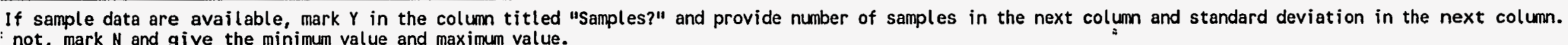
not, mark $N$ and give the minimum value and maximum value.

iditional information or explanations (indicate pertinent contaminant) 
- each contaminant, complete at least one line on the following table. If any entries for that contaminant vary by year, fill out additional lines as needed

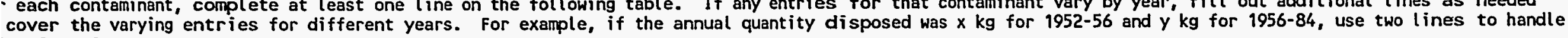
is situation.

\begin{tabular}{|c|c|c|c|c|c|c|c|c|c|c|c|}
\hline Radionuclide & Physical Form & Chemical Form & $\begin{array}{l}\text { (A)nnual/(T)otal } \\
\text { Quant ity }\end{array}$ & Unit & $\begin{array}{l}\text { Begin } \\
\text { Year }\end{array}$ & $\begin{array}{l}\text { End } \\
\text { Year }\end{array}$ & $\begin{array}{l}\text { Fore } \\
\text { cast }\end{array}$ & $\begin{array}{l}\text { Samp } \\
\text { les? }\end{array}$ & $\begin{array}{c}\text { Minimum } \\
\text { Value/\#Semp }\end{array}$ & $\begin{array}{c}\text { Maximum } \\
\text { Value/STD }\end{array}$ & $\begin{array}{l}\text { Basis for } \\
\text { Uncertainty }\end{array}$ \\
\hline Pu-239 & Unknown. & Unknown. & T. .00134700000000 & Cl & 2003 & 2003 & $Y$ & N & & & \\
\hline Pu-240 & Unknown. & Unknown. & $T .00014220000000$ & $\mathrm{CI}$ & 2003 & 2003 & $Y$ & N & & & \\
\hline Pu-241 & Unknown. & Unknown. & $T .14720000000000$ & CI & 2003 & 2003 & $Y$ & $N$ & & & \\
\hline$A m-241$ & Unknown. & Unknown. & T. .00067360000000 & CI & 2003 & 2003 & $Y$ & $N$ & & & \\
\hline $\mathrm{Cm}-242$ & Unknown. & Unknown. & T. .00067360000000 & $\mathrm{Cl}$ & 2003 & 2003 & $Y$ & $N$ & & & \\
\hline $\mathrm{cm}-244$ & Unknown. & Unknown. & $r .00062370000000$ & CI & 2003 & 2003 & $Y$ & N & & & \\
\hline & & & & & & & & & & & \\
\hline & & & & & & & & & & & \\
\hline & & & & & & & & & & & \\
\hline & & & & & & & & & & & \\
\hline & & & & & & & & & & & * \\
\hline
\end{tabular}

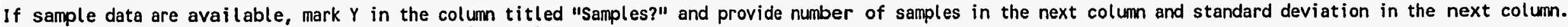
not, mark $N$ and give the minimum value and maximum value.

iditional information or explanations (indicate pertinent contaminant) 
Type of source of information: heck box)

] RWMIS [ ] other database sample analysis data operating records [ ] interview ] expert judgment [ ] reports generator forecasts other

Do the estimates of contaminant antities in Part $C$ and $D$ represent:

] best estimate

worst case

] other

Do the data conflict with RWMIs? (Historical or Present Data Only) ] no

] yes

Major unknowns in inventories of intaminants:

identified beta gamma (U-B-G) was known. The Ci content is unknown.
2. Details concerning source (names, report no., dates, etc.) SJK-005-93, S.J. Keating's letter to V.C. Randall on November 17, 1993, "Test Reactor Area (TRA) Radioactive Waste Forecasts".

4. If other than best estimate, explain why:

6. If yes, explain why:

A portion of or all of the measurements were assumed to be taken using a G-M detector which only measures gamma emitters; beta emitters have been added to the RWMIS activity based on scaling factors. Therefore, the total activity will be greater than that in RWMIS.

8. Key assumptions used to deal with the unknowns: $\mathrm{U}-\mathrm{B}-\mathrm{G}$ radionuclides were chosen based on EPRI NP-5077 for dry active waste for PWRs. The PWR similar to ATR, the reactor at TRA. The radionuclides chosen were based on a combination of the following: cross section, half lives, and fission products. This stream is a combination of what is reported in S.J. Keating letter to V.C. Randall, SJK-005-93 "Test Reactor Area (TRA) Radioactive Wastes Forecasts" November 17, 1993 and averaging 1984-1993 noncompactible waste. The present waste average was multiplied by 10 to reflect the 10 projected years. 
DATA INPUT FOR HISTORICAL DATA TASK FOR RWMC SUBSURFACE DISPOSAL AREA

\section{PART A - GENERAL INFORMATION PDT - 300}

. Preparer: Amaro, C.

3. Generator: TRA

(area or contractor - use code from attached list)

j. Number of waste stream from this facility: $1 \mathrm{P}$

7. Type of radioactive waste (check box):

[ ] TRU or suspect TRU

[X] LLW

] non-radioactive

3. Actual years disposed of at SDA: starting year 1995 Ending year 2000

10. Comments (specify number of pertinent question):
2. Date prepared: $07 / 16 / 94$

4. Particular facility: 670

(building number - use code from attached list)

6. Waste stream:

Beryllium block and shims from ATR.

9. Waste stream volume:

Amount

648.0000 Units Cubic meters.

Check box: [ ] annual or $[\mathrm{x}]$ total over all years

Check box: [ ] container volume or [X] waste volume 
General physical form (see attached list) 2. Details on physical form(particularly confinement related) X] other (specify)

eryllium.

\section{Chemical form:}

metal.

Waste container type (see attached list) ther.

Comments (specify number of pertinent question):
4. Inner packaging: [ ] plastic bag [X] plastic liner

[ ] metal liner [ ] none [ ] other (specify)

6. Other characteristics of interest: 


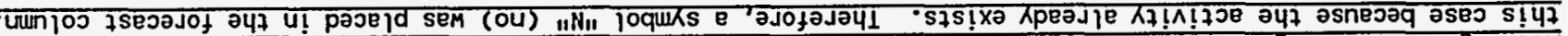

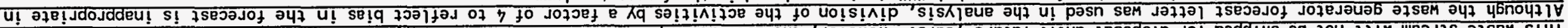

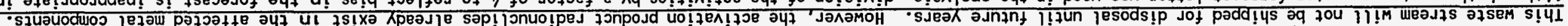

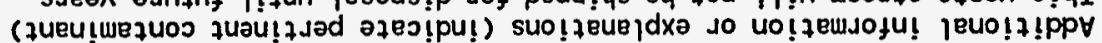

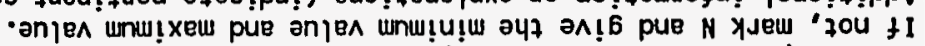

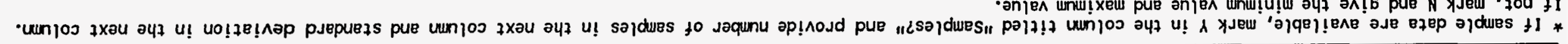

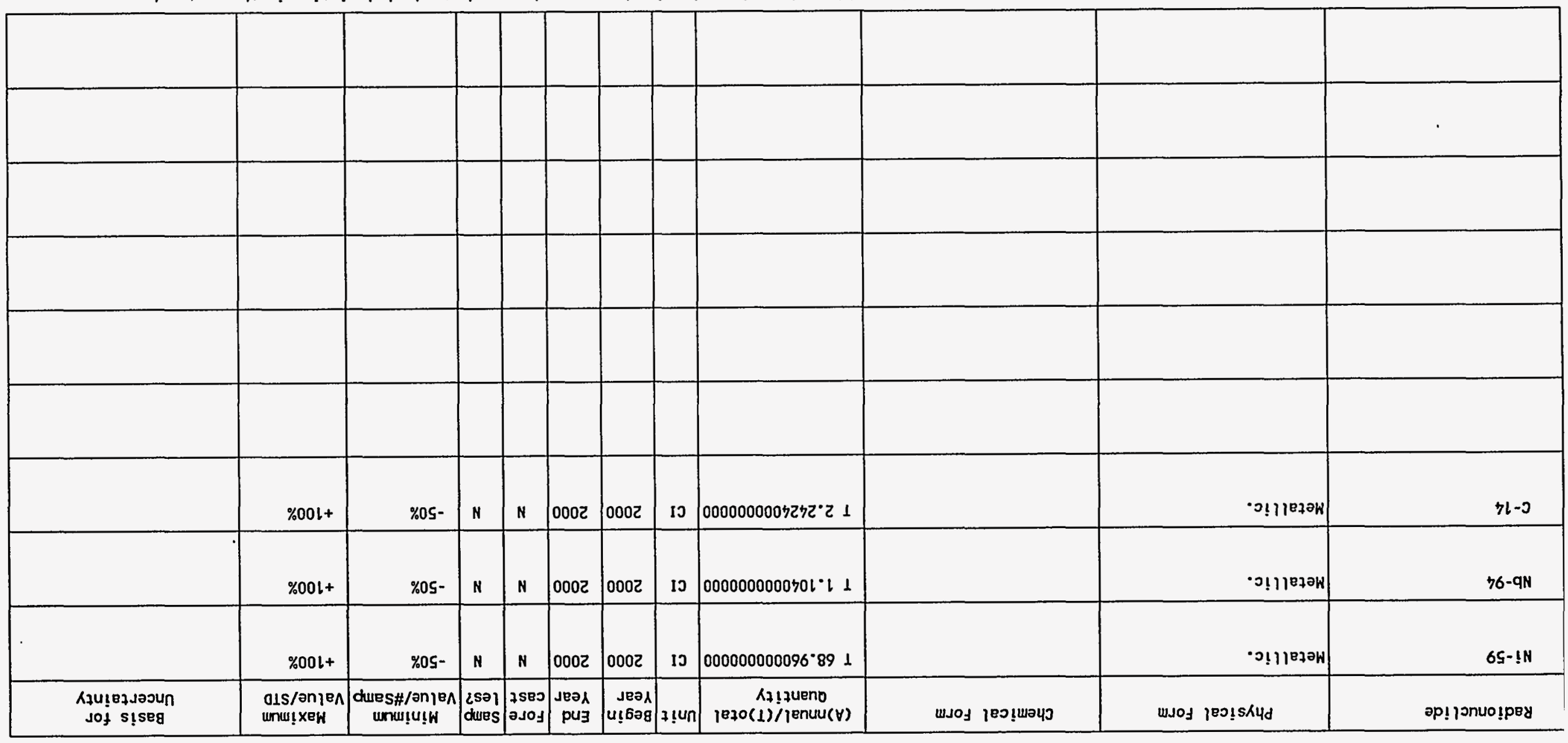

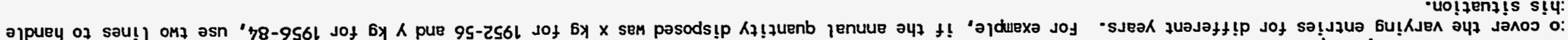

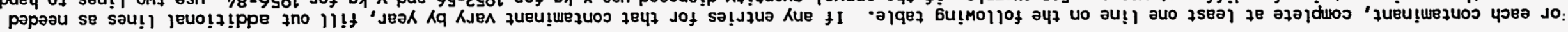


Type of source of information: heck box)

RWMIS [ ] other database

sample analysis data

operating records [X] interview

expert judgment [ ] reports

generator forecasts

other

Do the estimates of contaminant

antities in Part $C$ and $D$ represent:

best estimate

worst case

other

Do the data conflict with RWMIS?

(Historical or Present Data only)

] no

yes

Major unknowns in inventories of ntaminants:

ne.
2. Details concerning source (names, report no., dates, etc.) See Continuation Page of Part E.

4. If other than best estimate, explain why:

6. If yes, explain why:

8. Key assumptions used to deal with the unknowns: The short-lived $\mathrm{Cr}-5 \mathrm{I}$ and Fe-59 were deleted, based on assuming that they will have been almost completely decayed by the time the waste is buried, after storage for several years.

on forecasted waste, totals on the individual radionuclides can't match the forecasted totals. Twelve shipments each with two blocks for the year 1995. Four shipments each with four outer shims for the year 2000 . 


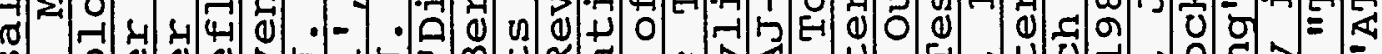

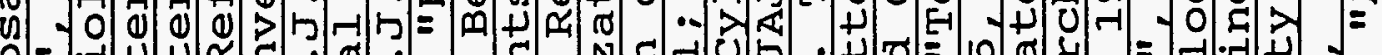

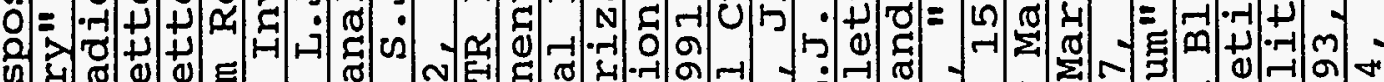
U Q

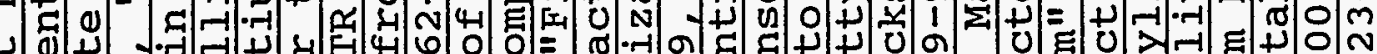

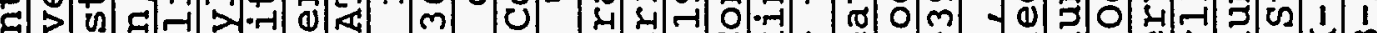

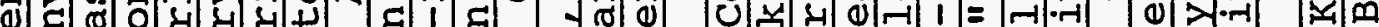
(A

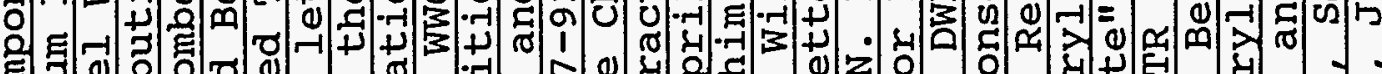

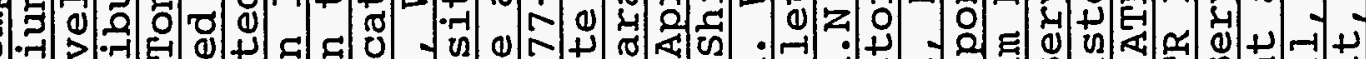

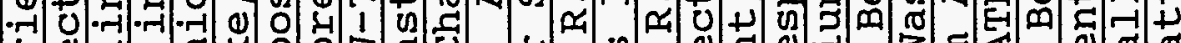

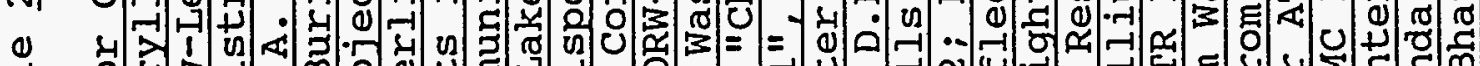
O t)

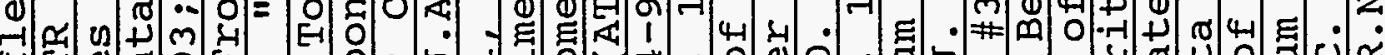

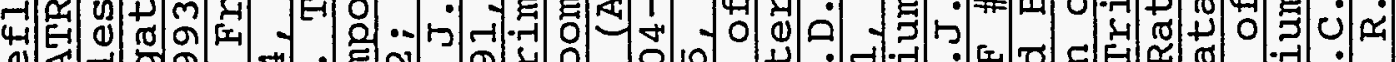

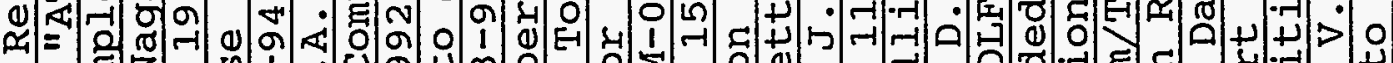

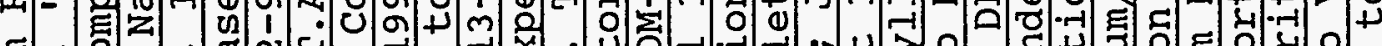

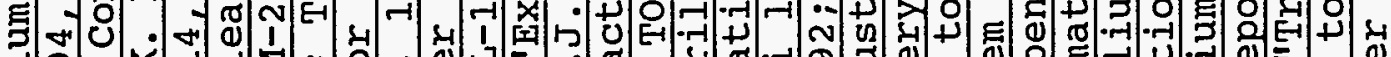

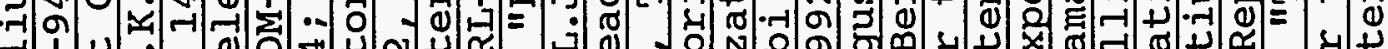
-1

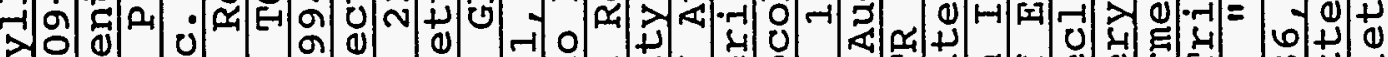

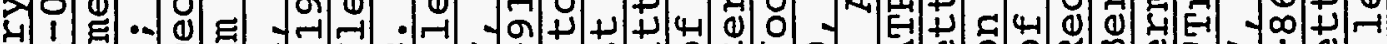
0.

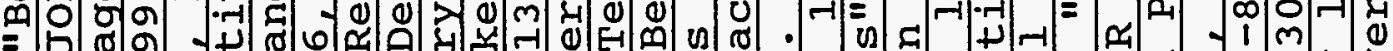

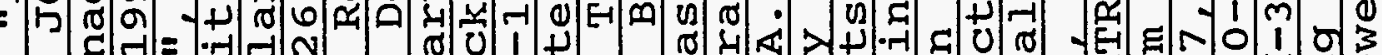
1 U m) 9)

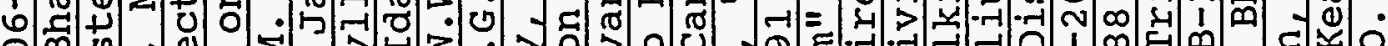

이에 1. b Z Z

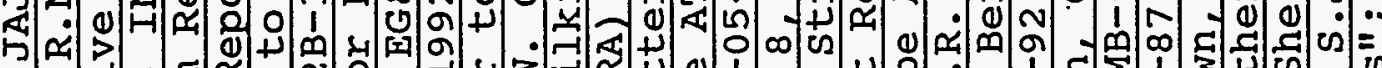
-

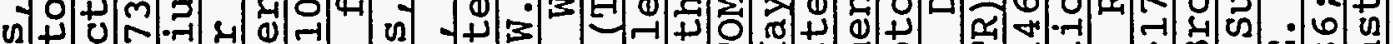

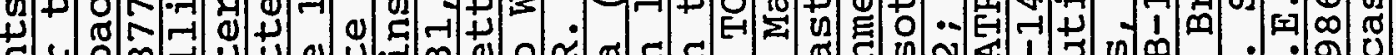

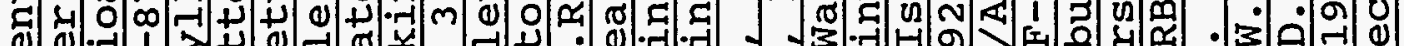
(3) d (1)

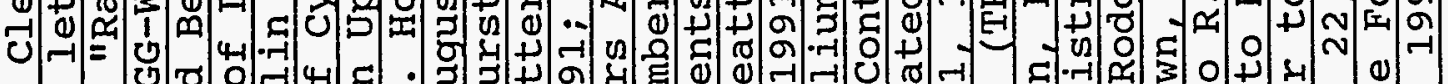
- 4 -

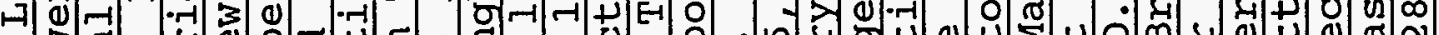

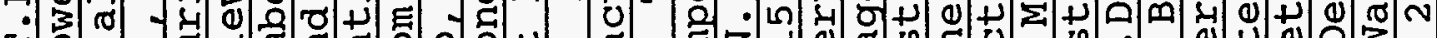
E-1 - J

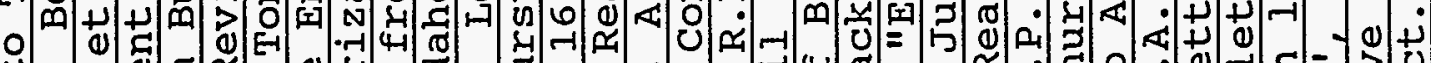
+0 4 u u 0)

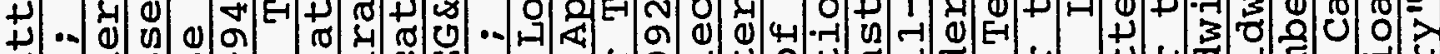

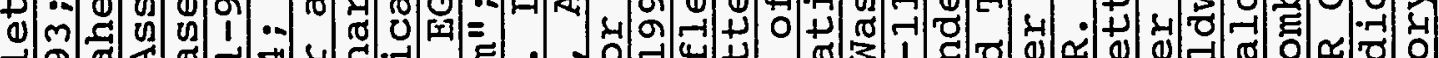
न1 の

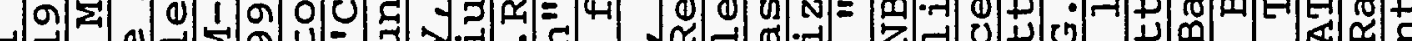

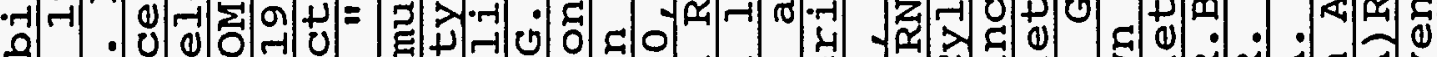

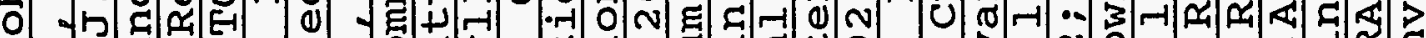
U઼

$\triangleright$. .

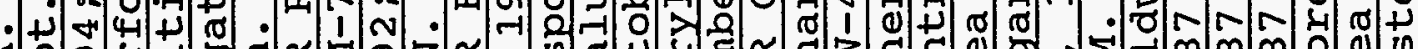

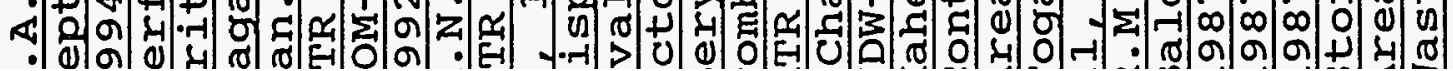


$\ldots+\ldots$

...

. 
DATA INPUT FOR HISTORICAL DATA TASK FOR RWMC SUBSURFACE DISPOSAL AREA

ART A - GENERAL INFORMATION RDT - 257

- Preparer: Rhodes, Donald W.

- Generator: WER

area or contractor - use code from attached list)

Number of waste stream from this facility: IR

- Type of radioactive waste (check box):

] TRU or suspect TRU

X] LLW

] non-radioactive

- Actual years disposed of at SDA: tarting year 1987 Ending year 1991

o. Comments (specify number of pertinent question):
2. Date prepared:07/06/94

4. Particular facility: COM

(building number - use code from attached list)

6. Waste stream:

Compactible wastes with low radionuclide content received from all of the facilities at the INEL for size reduction by compaction.

9. Waste stream volume:

Amount 826.0000 Units Cubic meters.

check box: [ ] annual or [X] total over all years

Check box: [X] container volume or [ ] waste volume 
General physical form (see attached list) 2. Details on physical form(particularly confinement related) er scrap metals. Metal, filters, cloth, paper, plastic. Waste is compacted using a 200-ton compacter to reduce the volume prior to disposal in the RWMC.

\section{Chemical form:}

bon steel, stainless steel, fiberglass Iters), plastic (halogenated and -halogenated), and cellulose (wood).

Waste container type (see attached Iist) al box.

4. Inner packaging: [ ] plastic bag [ ] plastic liner

[ ] metal liner [X] none [ ] other (specify)

6. Other characteristics of interest:

A total of 292 shipments was made to the RWMC.

Comments (specify number of pertinent question):

1. Also $21,22,23,42$, and 44 .

5. Also BIN. 
each contaminant, complete at least one line on the following table. If any entries for that contaminant vary by year, fill out additional lines as needed

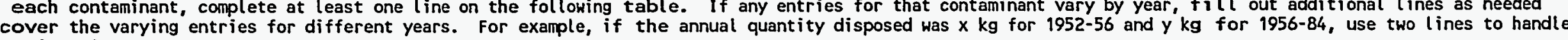
cover the varying entries for differe.
situation.

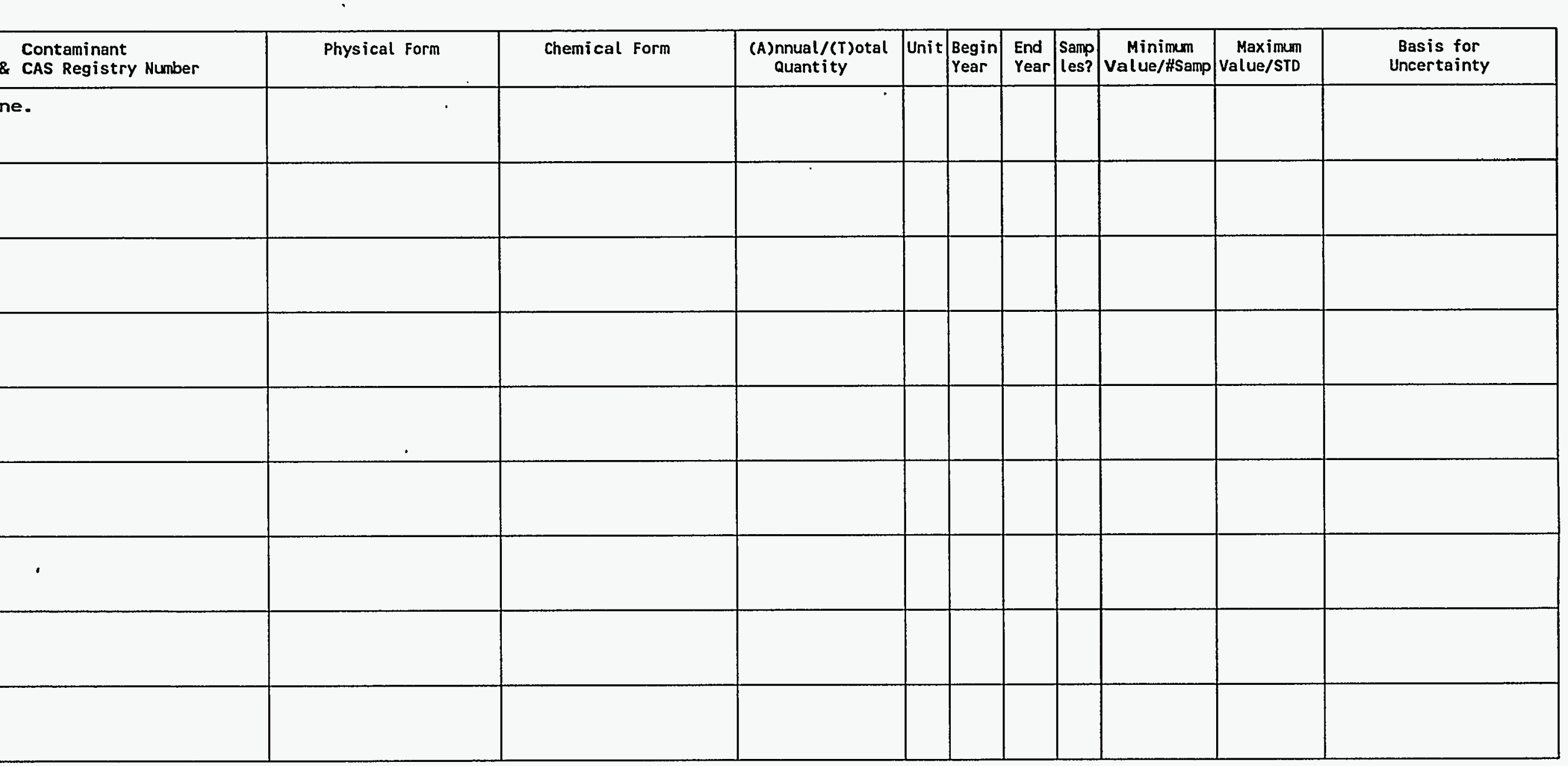

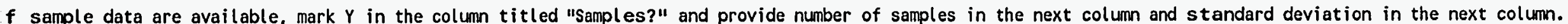
not, mark $N$ and give the minimum value and maximum value.

itional information or explanations (indicate pertinent contaminant) 
each contaminant, complete at least one line on the following table. If any entries for that contaminant vary by year, fill out additional lines as needed over the varying entries for different years. For example, if the annual quantity disposed was $x \mathrm{~kg}$ for $1952-56$ and $y \mathrm{~kg}$ for $1956-84$, use two lines to handle situation.

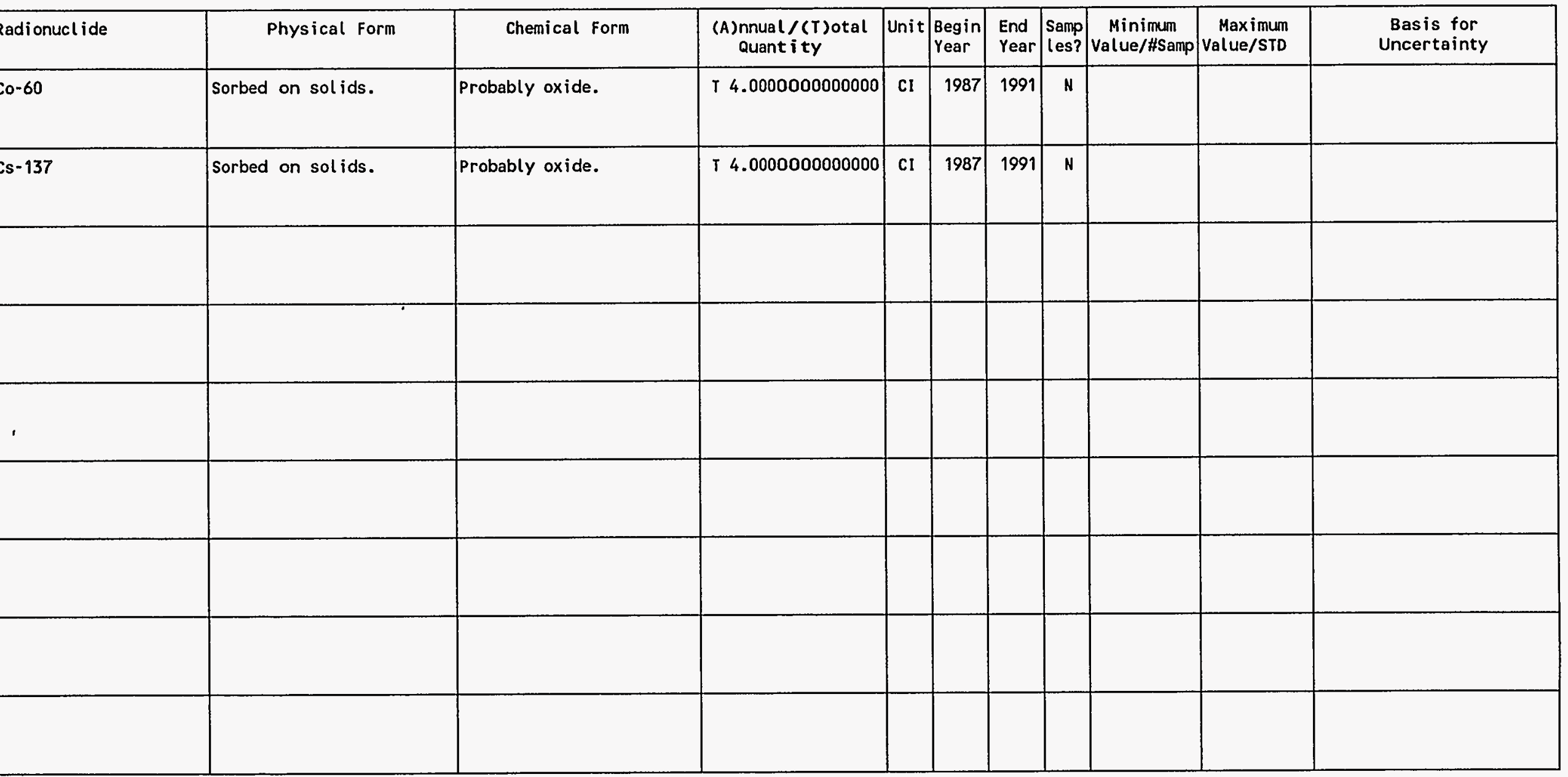

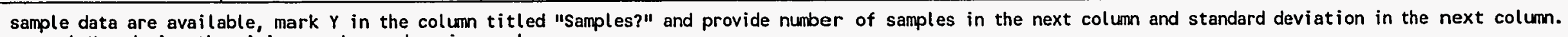

ot, mark $N$ and give the minimum value and maximum value.

tional information or explanations (indicate pertinent contaminant)

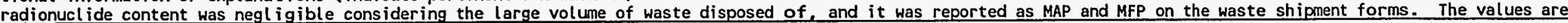
rted here as $\mathrm{Co}-60$ and $\mathrm{cs}-137$, since these would be the radionuclides most easily measured by radiation instruments. 
Type of source of information: heck box)

:] RWMIS [X] other database

] sample analysis data

] operating records [ ] interview

:] expert juagment [ ] reports

other

Do the estimates of contaminant lantities in Part $C$ and $D$ represent:

i] best estimate

] worst case

] other

Do the data conflict with RWMIS? (Historical or Present Data only) I] no

] yes

Major unknowns in inventories of ontaminants :

idividual radionuclides were not reported.
2. Details concerning source (names, report no., dates, etc.) RWMIS printout for 1984-93. Tech Note data compiled from RWMIS by C.J. Barnard and M.J. Schlafman. Report WM-F1-81-026 Rev. 9, June 1993, SAR for the waste Experimental Reduction Facility.

4. If other than best estimate, explain why:

6. If yes, explain why:

8. Key assumptions used to deal with the unknowns: The MAP and MFP were considered to be Co-60 and Cs-137 respectively. 
DATA INPUT FOR HISTORICAL DATA TASK FOR RWMC SUBSURFACE DISPOSAL AREA

\section{RT A - GENERAL INFORMATION RDT - 256}

Preparer: Rhodes, Donald W.

Generator: WER

rea or contractor - use code from attached list)

Number of waste stream from this facility: IR

Type of radioactive waste (check box):

TRU or suspect TRU

LLW

non-radioactive

Actual years disposed of at SDA:

arting year 1987 Ending year 1993
2. Date prepared: 07/06/94

4. Particular facility: INC

(building number - use code from attached list)

6. Waste stream:

Waste received from all facilities at the INEL was incinerated prior to disposal. The ash was disposed of at the RWMC.

9. Waste stream volume:

Amount

162.0000 Units Cubic meters.

Check box: [ ] annual or [X] total over all years

Check box: [X] container volume or [ ] waste volume

\section{Comments (specify number of pertinent question):}

6. Waste was required to meet the strict criteria with respect to radionuclide and hazardous material content before it could be incinerated. 
General physical form (see attached list) sot, ash.

] other (specify)

Chemical form:

ilica, calcium, and silicate.

. Waste container type (see attached list) tal barrel.

Comments (specify number of pertinent question) :

1. Also $11,42,41$, and 44 .

5. Also BXW, MWB, BIN, TX4, and BXM.
2. Details on physical form (particularly confinement related) Waste was primarily ash from the incineration of combustible materials, but also sometimes was solidified in concrete before disposal at the RWMC. Sometimes minor amounts of glass or metal chips were part of the waste.

4. Inner packaging: [X] plastic bag [ ] plastic liner [ ] metal liner [ ] none [ ] other (specify)

6. Other characteristics of interest: 
Type of source of information: heck box)

RWMIS

[X] other database

sample analysis data

operating records [ ] interview

] expert judgment [ ] reports

other

Do the estimates of contaminant lantities in Part $C$ and $D$ represent: ] best estimate

] worst case

other

Do the data conflict with RWMIS?

(Historical or Present Data only) no

] yes

Major unknowns in inventories of ntaminants :

jor radionuclide contamination reported MAP and MFP
2. Details concerning source (names, report no., dates, etc.) RWMIS printout 1984-93. Tech Note data compiled from RWMIS

by C.J. Barnard and M.J. Schlafman. Report WM-F1-81-026 Rev. 5 , September 1985 , SAR for the Waste Experimental Reduction Facility.

4. If other than best estimate, explain why:

6. If yes, explain why:

8. Key assumptions used to deal with the unknowns: MAP and MFP assumed to be Co-60 and Cs-137 respectively. 
DATA INPUT FOR HISTORICAL DATA TASK FOR RWMC SUBSURFACE DISPOSAL AREA

\section{RT A - GENERAL INFORMATION RDT - 258}

Preparer: Rhodes, Donald W.

Generator: WER

irea or contractor - use code from attached list)

Number of waste stream from this facility: IR

Type of radioactive waste (check box):

] TRU or suspect TRU

r] LLW

] non-radioactive

Actual years disposed of at SDA: :arting year 1985 Ending year 1991

). Comments (specify number of pertinent question):
2. Date prepared: $07 / 06 / 94$

4. Particular facility: MET

(building number - use code from attached list)

6. Waste stream:

Primarily structural materials and equipment that have been reduced in size at the WERF facility to minimize the volume prior to disposal.

9. Waste stream volume:

Amount

879.0000 Units Cubic meters.

Check box: [ ] annual or [X] total over all years

Check box: [X] container volume or [ ] waste volume 
General physical form (see attached 1 ist) ier scrap metals. other (specify)

Chemical form:

:al, carbon steel, stainless steel, and iminum.

Waste container type (see attached list) den box.

Comments (specify number of pertinent question): 1. Also 21 and 42 .

5. Also BLX, ING, $\mathrm{O}, \mathrm{Pb} 2$ and $\mathrm{Pb} 3$.
2. Details on physical form(particularly confinement related) Structural materials are dismantled and cut up with a plasma arc torch so they will use up less storage volume at the RWMC. Some metals were melted and cast into ingot.

4. Inner packaging: [ ] plastic bag [ ] plastic liner [ ] metal liner [X] none [ ] other (specify)

6. Other characteristics of interest: 


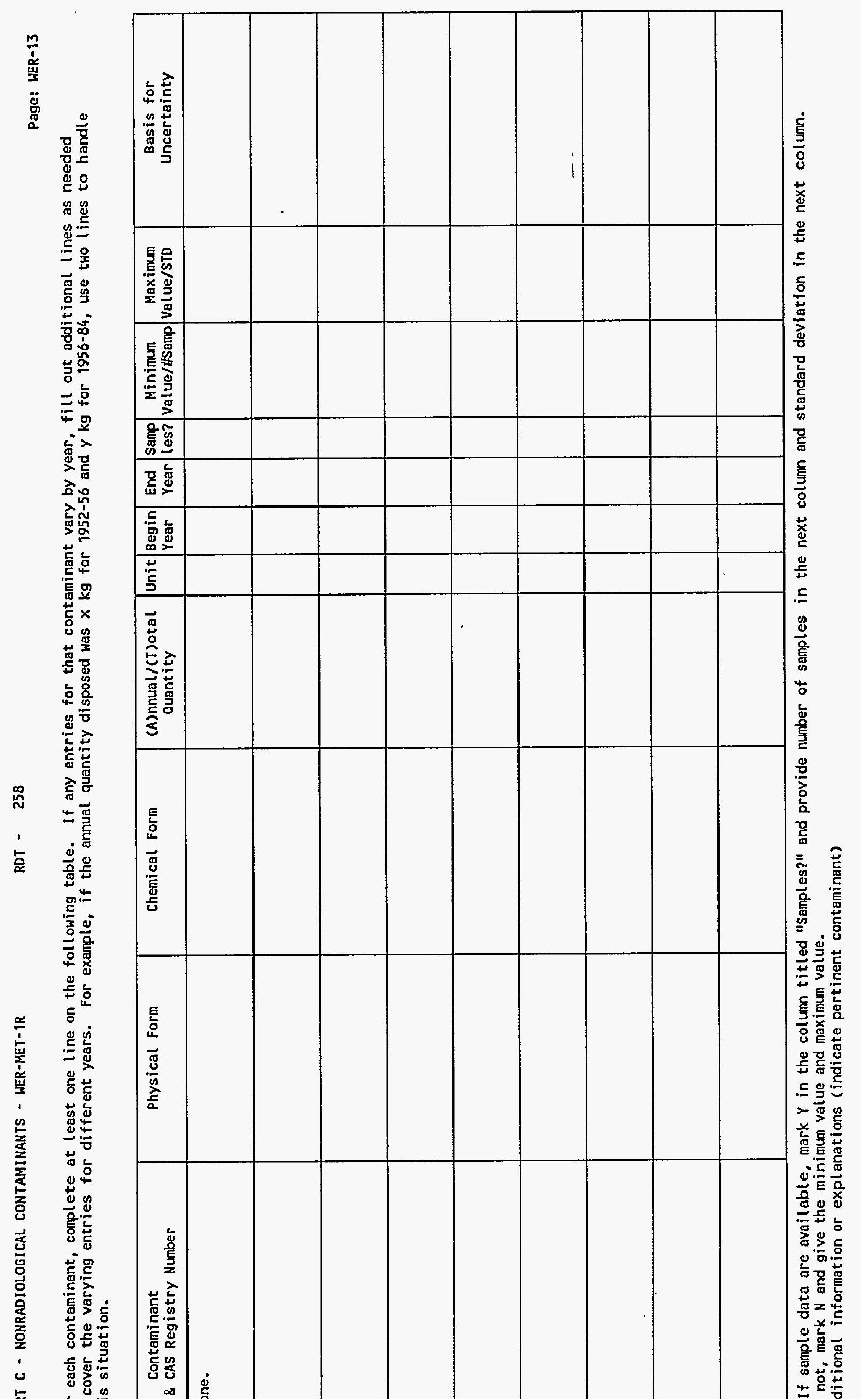


- Type of source of information: sheck box)

K] RWMIS [X] other database

] sample analysis data

] operating records [ ] interview

x] expert judgment [X] reports

] other

Do the estimates of contaminant dantities in Part $C$ and $D$ represent:

$x]$ best estimate

] worst case

] other

Do the data conflict with RWMIS? (Historical or Present Data only) $x]$ no

] yes

- Major unknowns in inventories of ontaminants:

he radionuclide contaminants are reported S MAP and MFP.
2. Details concerning source (names, report no., dates, etc.) RWMIS printout for 1984-93. Tech Note data compiled from RWMIS by C.J. Barnard and M.J. Schlafman. Report WM-F1-81-026, SAR for the Waste Experimental Reduction Facility, Rev. 5, September 1985.

4. If other than best estimate, explain why:

6. If yes, explain why:

8. Key assumptions used to deal with the unknowns:

Assumed that the major radionuclides were Co-60 and Cs-137 for MAP and MFP respectively. 


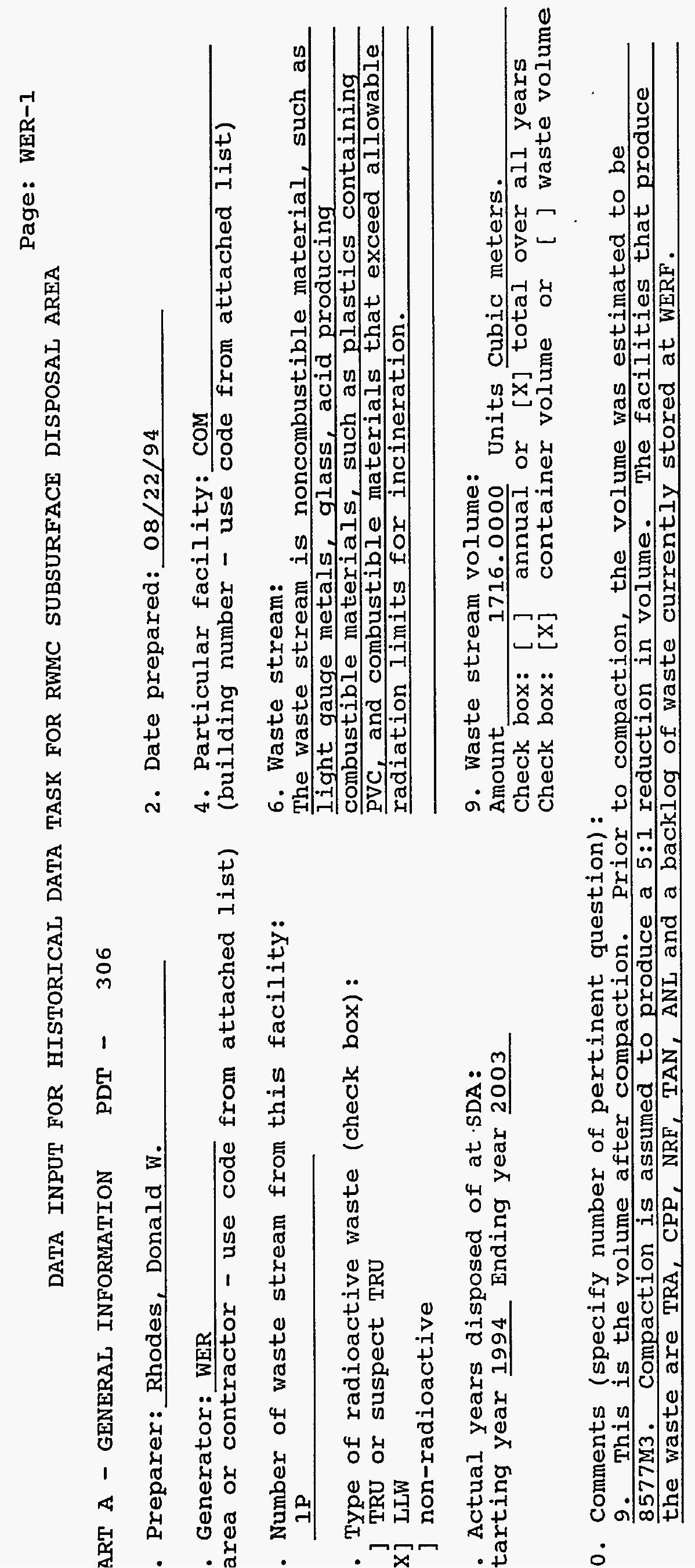


General physical form (see attached list) 2. Details on physical form(particularly confinement related) ier scrap metals. Light gauge metals, wood, plastic, glass. In general, other (specify) noncombustibles with low radioactive contamination.

\section{Chemical form:}

al (stainless steel, carbon steel iminum) halogen-containing plastics, and lulose (wood).

Waste container type (see attached list) :al box.

Comments (specify number of pertinent question) :

1. Also 42 and 44 .

4. Inner packaging: [ ] plastic bag [ ] plastic liner

[ ] metal liner $[X]$ none [ ] other (specify)

6. Other characteristics of interest: 
each contaminant, complete at least one line on the following table. If any entries for that contaminant vary by year, fill out additional lines as needed

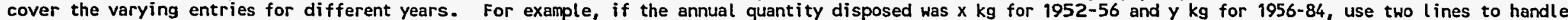
s situation.

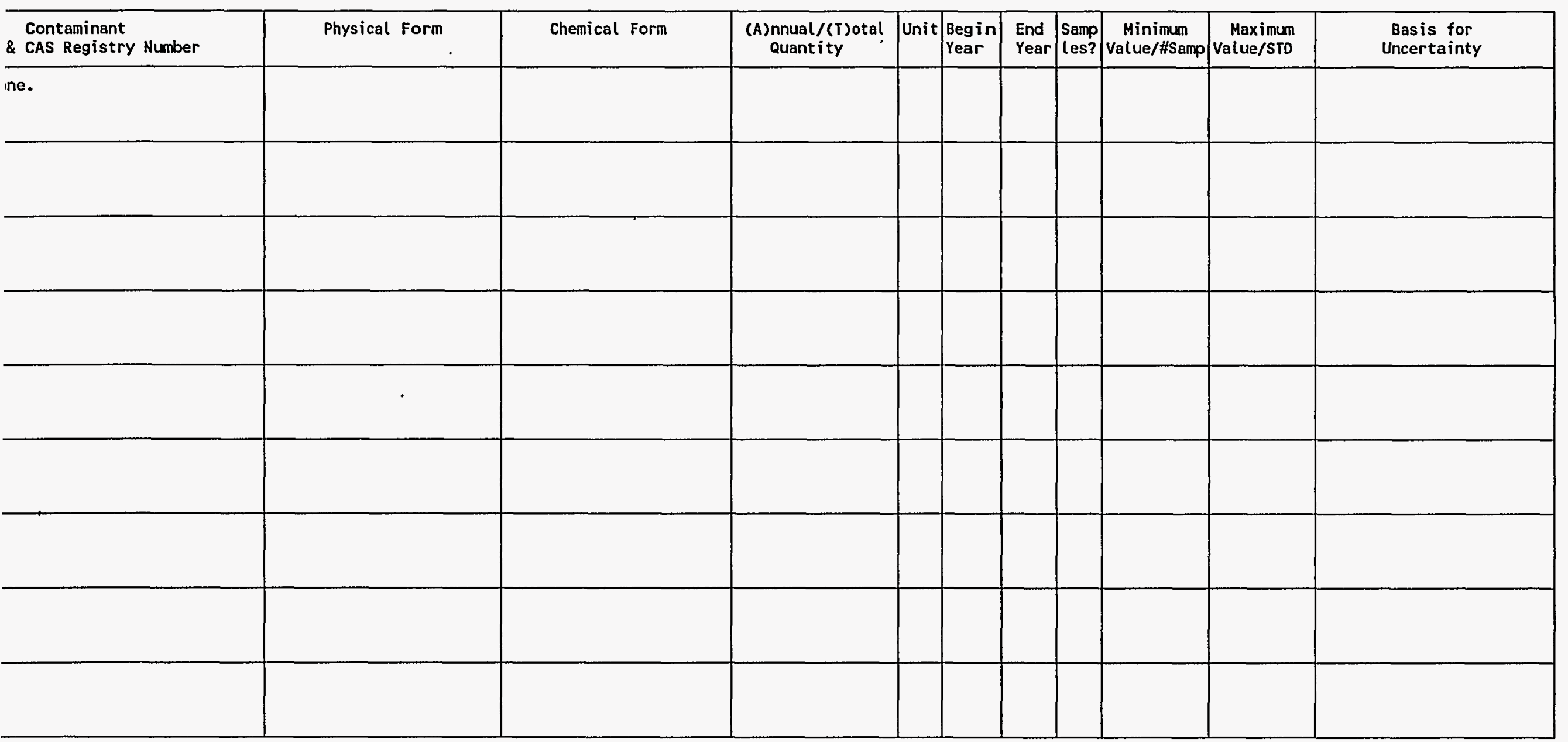

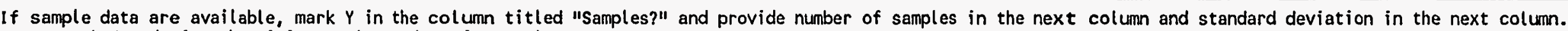
not, mark $N$ and give the minimum value and maximum value.

ditional information or explanations (indicate pertinent contaminant) 
ach contaminant, complete at least one line on the following table. If any entries for that contaminant vary by year, fill out additional lines as needed ver the varying entries for different years. For example, if the annual quantity disposed was $x \mathrm{~kg}$ for $1952-56$ and $y \mathrm{~kg}$ for $1956-84$, use two lines to handle situation.

\begin{tabular}{|c|c|c|c|c|c|c|c|c|c|c|c|}
\hline adionuclide & Physical form & Chemical Form & $\begin{array}{l}\text { (A)nnual } /(T) \text { otal } \\
\text { Quant ity }\end{array}$ & Unit & $\begin{array}{l}\text { Begin } \\
\text { Year }\end{array} \mid$ & $\begin{array}{l}\text { End } \\
\text { Year }\end{array}$ & $\begin{array}{l}\text { Fore } \\
\text { cast }\end{array}$ & $\begin{array}{l}\text { Samp } \\
\text { les? }\end{array}$ & $\begin{array}{c}\text { Minimum } \\
\text { Value/\#Samp }\end{array}$ & $\begin{array}{r}\text { Maximum } \\
\text { Value/STD }\end{array}$ & $\begin{array}{l}\text { Basis for } \\
\text { Uncertainty }\end{array}$ \\
\hline$e-55$ & Occluded in metal. & Metal. & T 9.0600000000000 & $\mathrm{Cl}$ & 1994 & 2003 & $Y$ & N & & & \\
\hline$i-63$ & Occluded in metal. & Metal. & T 1.6200000000000 & CI & 1994 & 2003 & $Y$ & N & & & \\
\hline $0-60$ & Occluded in metal. & Metal. & T 40.110000000000 & CI & 1994 & 2003 & $Y$ & N & & & \\
\hline $0-58$ & Occluded in metal. & Metal. & T 5.3800000000000 & $\mathrm{CI}$ & 1994 & 2003 & $Y$ & N & & & \\
\hline $1 n-54$ & Occluded in metal. & Metal. & T 3.3700000000000 & $\mathrm{CI}$ & 1994 & 2003 & Y & N & & & \\
\hline$r-90$ & Sorbed on waste solids. & Probably oxides. & r 22.310000000000 & CI & 1994 & 2003 & Y & N & & & \\
\hline-90 & Sorbed on waste solids. & Probably oxides. & T 22.310000000000 & CI & 1994 & 2003 & $Y$ & N & & & \\
\hline$u-154$ & Sorbed on waste solids. & Probably oxides. & T. .19000000000000 & CI & 1994 & 2003 & $Y$ & N & & & \\
\hline-3 & Sorbed on waste solids. & Probably oxides. & T 4.4700000000000 & CI & 1994 & 2003 & $Y$ & N & $-50 \%$ & $+50 \%$ & Professional judgment. \\
\hline
\end{tabular}

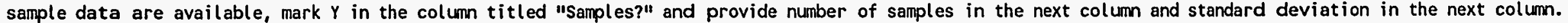

ot, mark $N$ and give the minimum value and maximum value.

tional information or explanations (indicate pertinent contaminant)

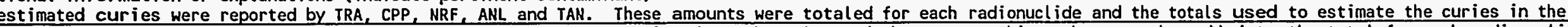

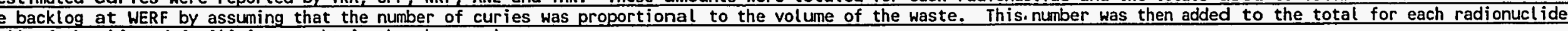
all of the listed facilities to obtain the above values. 
- each contaminant, complete at least one line on the following table. If any entries for that contaminant vary by year, fill out additional lines as needed

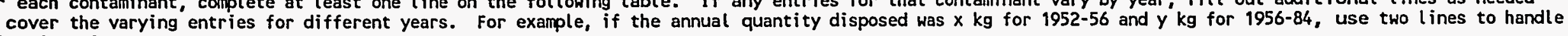
is situation.

\begin{tabular}{|c|c|c|c|c|c|c|c|c|c|c|c|}
\hline Radionuclide & Physical Form & Chemical Form & $\begin{array}{l}\text { (A)nnual/(T)otal } \\
\text { Quantity }\end{array}$ & Unit & $\begin{array}{l}\text { Begin } \\
\text { Year }\end{array}$ & $\begin{array}{l}\text { End } \\
\text { Year }\end{array}$ & $\begin{array}{l}\text { Fore } \\
\text { cast }\end{array}$ & $\begin{array}{l}\text { Samp } \\
\text { les? }\end{array}$ & $\begin{array}{c}\text { Minimum } \\
\text { Value/\#Samp }\end{array}$ & $\begin{array}{r}\text { Maximum } \\
\text { Value/STD }\end{array}$ & $\begin{array}{l}\text { Basis for } \\
\text { Uncertainty }\end{array}$ \\
\hline Ru- 106 & Sorbed on waste solids. & Probably oxides. & $T .13000000000000$ & CI & 1994 & 2003 & $Y$ & N & & & \\
\hline$R h-106$ & Sorbed on waste solids. & Probably oxides. & T. .13000000000000 & CI & 1994 & 2003 & $Y$ & N & & & $\cdot$ \\
\hline Eu-152 & Sorbed on waste solids. & Probably oxides. & T 5.5000000000000 & $\mathrm{CI}$ & 1994 & 2003 & $\mathbf{Y}$ & N & & & \\
\hline Cs -134 & Sorbed on waste solids. & Probably oxides. & T 6.5900000000000 & CI & 1994 & 2003 & $\mathbf{Y}$ & N & & & \\
\hline Cs-137 & Sorbed on waste solids. & Probably oxides. & T 41.150000000000 & CI & 1994 & 2003 & $Y$ & N & & & \\
\hline $\mathrm{Ce}-144$ & Sorbed on waste solids. & Probably oxides. & T 1.3900000000000 & CI & 1994 & 2003 & $Y$ & N & & & \\
\hline $\operatorname{Pr}-144$ & Sorbed on waste solids. & Probably oxides. & T 1.3900000000000 & CI & 1994 & 2003 & $Y$ & N & & & \\
\hline Eu-155 & Sorbed on waste solids. & Probably oxides. & T.74000000000000 & $\mathrm{CI}$ & 1994 & 2003 & $Y$ & N & & & \\
\hline $1-129$ & Sorbed on waste solids. & Probably oxides. & $T .00200000000000$ & $\mathrm{CI}$ & 1994 & 2003 & $Y$ & $\mathbf{N}$ & $-50 \%$ & $+50 \%$ & Professional judgment. \\
\hline
\end{tabular}

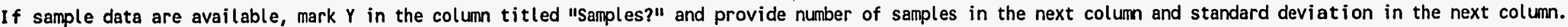

not, mark $N$ and give the minimum value and maximum value.

ditional information or explanations (indicate pertinent contaminant)

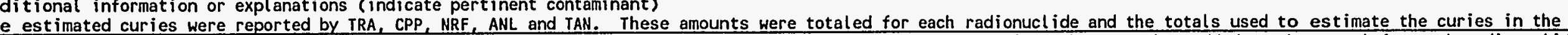

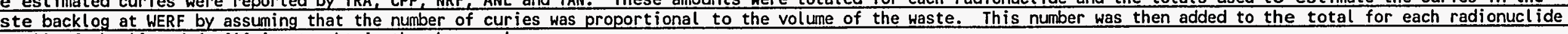
$r$ all of the listed facilities to obtain the above values. 
each contaminant, complete at least one line on the following table. If any entries for that contaminant vary by year, fill out additional lines as needed

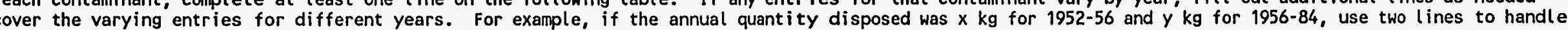
situation.

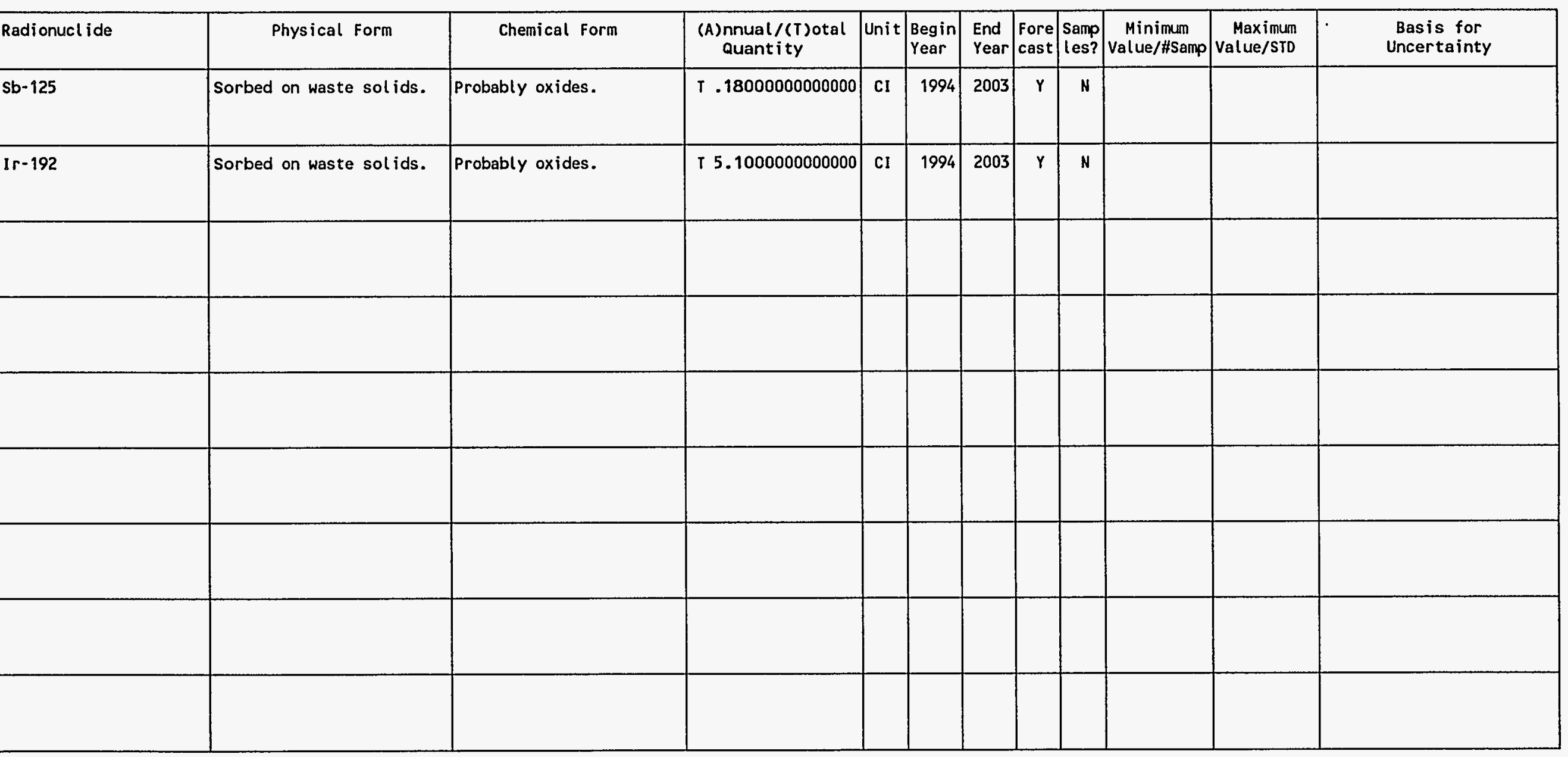

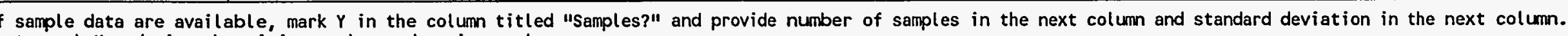
ot, mark $N$ and give the minimum value and maximum value.

tional information or explanations (indicate pertinent contaminant)

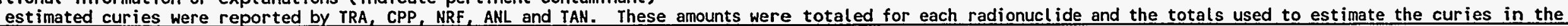

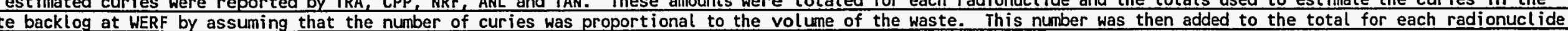
all of the listed facilities to obtain the above values. 
- Type of source of information: check box)

] RWMIS [ ] other database sample analysis data operating records $[X]$ interview $\mathrm{X}]$ expert judgment $[\mathrm{X}]$ reports

$\mathrm{X}$ ] generator forecasts

other

- Do the estimates of contaminant uantities in Part $C$ and $D$ represent:

$\mathrm{X}$ ] best estimate

] worst case

] other

- Do the data conflict with RWMIS?

(Historical or Present Data Only)

$\mathrm{X}] \mathrm{no}$

] Yes

- Major unknowns in inventories of :ontaminants:

incertain about future programs that will roduce the waste. Uncertain about startup lata for WERF.
2. Details concerning source (names, report no., dates, etc.) Report WERF SAR, Vol. 1, WM-F1-81-026 Rev.9, June 1993. Solid Radioactive Waste Forecast (RST-018-93) prepared by J.R. Hitz and approved by R.L. Skinner, 11/15/93. Conversation with J.L. Butler. See other sources on continuation pages.

4. If other than best estimate, explain why:

6. If yes, explain why:

8. Key assumptions used to deal with the unknowns: Assumed the anticipated programs that will produce the waste are valid. Assumed that WERF compaction will begin operation the early part of 1995. Disposal is assumed to begin in 1995. 
'est Reactor Area (TRA) Radioactive Waste Forecasts, SJK-005-93, Nov. 17, 1993. Solid Radioactive laste Forecast, KAK-16-93, NoV. 15, 1993. NRF Forecasts for Waste sent to WERF, 995-2003-PRL-15-94, Aug. 9, 1994. Solid Radioactive Waste Forecast for TAN, prepared by Mat ianister, Nov. 18, 1993. Solid Radioactive Waste Forecast for TAN prepared by David Gibbon, Nov. 6. 1993. Data Input for RPDT for RWMC SDA, R.P. Grant, Sept. 30, 1994. 
DATA INPUT FOR HISTORICAL DATA TASK FOR RWMC SUBSURFACE DISPOSAL AREA

IRT A - GENERAL INFORMATION PDT - 307

Preparer: Rhodes, Donald W.

Generator: WER irea or contractor - use code from attached list)

Number of waste stream from this facility: $1 \mathrm{P}$

Type of radioactive waste (check box): ] TRU or suspect TRU

(]) LLW

] non-radioactive

- Actual years disposed of at SDA: zarting year 1994 Ending year 2003
2. Date prepared: $08 / 22 / 94$

4. Particular facility: INC

(building number - use code from attached list)

6. Waste stream:

This waste stream is combustible waste that is scheduled to be incinerated at WERF or at a similar off-site facility.

\section{Waste stream volume:}

Amount

92.0000 Units Cubic meters.

Check box: [ ] annual or [X] total over all years

Check box: $[\mathrm{X}]$ container volume or $[\mathrm{]}$ waste volume

J. Comments (specify number of pertinent question):

9. This is the volume of ash that will be buried in the RWMC resulting from incineration of the combustible waste. Incineration is assumed to produce a 200:1 reduction in volume. The original volume, before incineration, was estimated to be $18,473 \mathrm{M} 3$. The facilities that will produce this stream are CPP, TRA, NRF, TAN, ANL and a backlog of waste currently stored at WERF. 


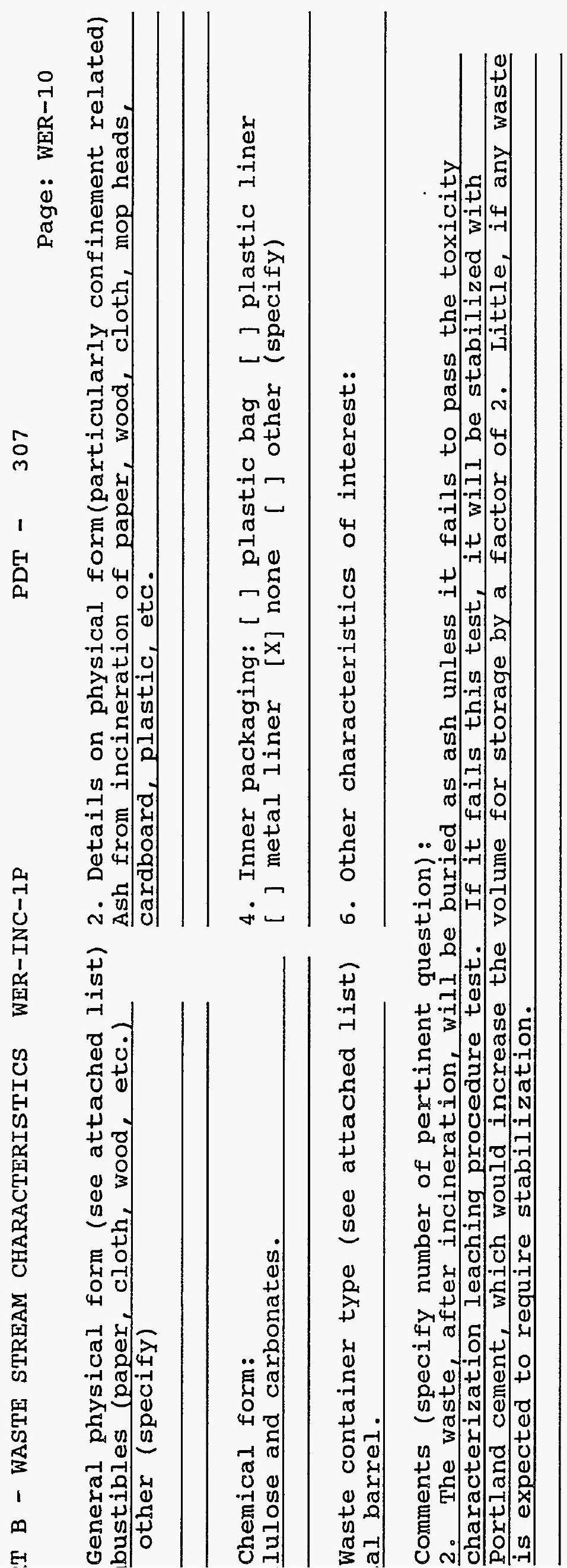


Ir each contaminant, complete at least one line on the following table. If any entries for that contaminant vary by year, fill out additional lines as needed

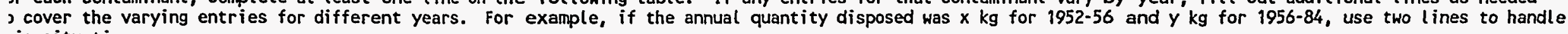
lis situation.

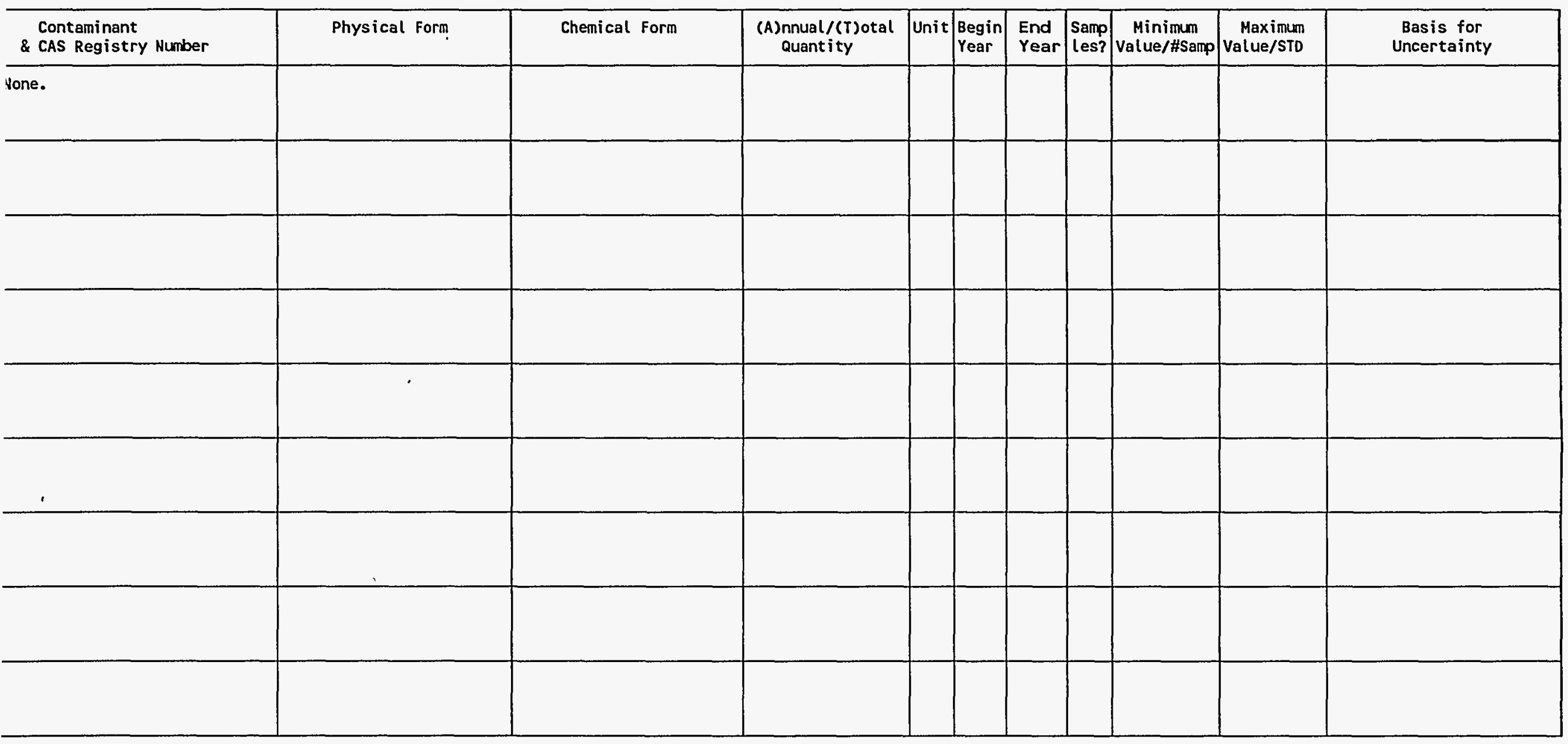

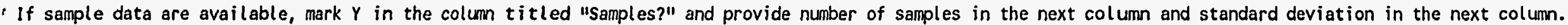
if not, mark $N$ and give the minimum value and maximum value.

idditional information or explanations (indicate pertinent contaminant) 
- each contaminant, complete at least one line on the following table. If any entries for that contaminant vary by year, fill out additional lines as needed

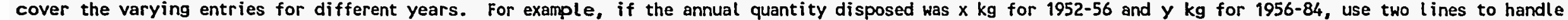
is situation.

\begin{tabular}{|c|c|c|c|c|c|c|c|c|c|c|c|}
\hline Radionuclide & Physical Form & Chemical Form & $\begin{array}{l}\text { (A)nnual/(T)otal } \\
\text { Quant ity }\end{array}$ & Unit & $\begin{array}{l}\text { Begin } \\
\text { Year }\end{array}$ & $\begin{array}{l}\text { End } \\
\text { Year }\end{array}$ & $\begin{array}{l}\text { Fore } \\
\text { cast }\end{array}$ & $\begin{array}{l}\text { Samp } \\
\text { les? }\end{array}$ & $\begin{array}{c}\text { Minimum } \\
\text { Value/\#Samp }\end{array}$ & $\begin{array}{r}\text { Maximum } \\
\text { Value/STD }\end{array}$ & $\begin{array}{l}\text { Basis for } \\
\text { Uncertainty }\end{array}$ \\
\hline Cs -134 & Sorbed on waste solids. & Probably oxides. & T 7.7100000000000 & $\mathrm{CI}$ & 1994 & 2003 & Y & N & & & \\
\hline Cs -137 & Sorbed on waste solids. & Probably oxides. & T 91.940000000000 & CI & 1994 & 2003 & Y & $\mathbf{N}$ & & & \\
\hline Ce- 144 & Sorbed on waste solids. & Probably oxides. & T 1.9000000000000 & $\mathrm{CI}$ & 1994 & 2003 & $Y$ & N & & & \\
\hline $\mathrm{Pr}-144$ & Sorbed on waste solids. & Probably oxides. & T 1.9000000000000 & $\mathrm{Cl}$ & 1994 & 2003 & $Y$ & N & & & \\
\hline$S b-125$ & Sorbed on waste solids. & Probably oxides. & T. .34000000000000 & CI & 1994 & 2003 & Y & N & & & \\
\hline I $r-192$ & Sorbed on waste solids. & Probably oxides. & T 5.9100000000000 & $\mathrm{CI}$ & 1994 & 2003 & Y & $\mathbf{N}$ & & & \\
\hline Eu-154 & Sorbed on waste solids. & Probably oxides. & T 6.5000000000000 & $\mathrm{Cl}$ & 1994 & 2003 & Y & $N$ & & & \\
\hline Eu-155 & Sorbed on waste solids. & Probably oxides. & T. .50000000000000 & CI & 1994 & 2003 & $Y$ & N & & & \\
\hline $1-129$ & Sorbed on waste solids. & Probably oxides. & $T .00400000000000$ & CI & 1994 & 2003 & $Y$ & $N$ & $-50 \%$ & $+50 \%$ & Professional judgment. \\
\hline
\end{tabular}

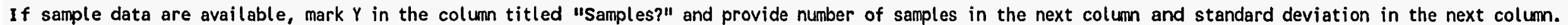
: not, mark $N$ and give the minimum value and maximum value.

Iditional information or explanations (indicate pertinent contaminant)

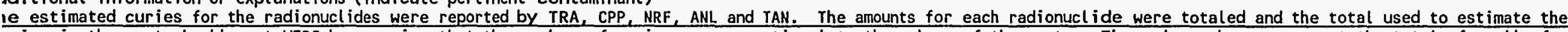

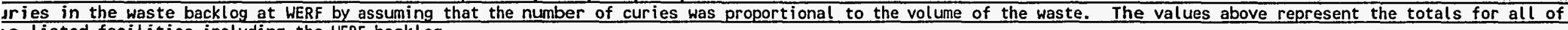
ie listed facilities including the WERF backlog. 
each contaminant, complete at least one line on the following table. If any entries for that contaminant vary by year, fill out additional lines as needed

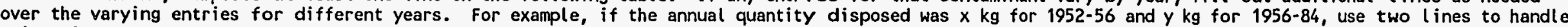
situation.

\begin{tabular}{|c|c|c|c|c|c|c|c|c|c|c|c|}
\hline Radionuclide & Physical Form & Chemical Form & $\begin{array}{l}\text { (A)nnual } /(\mathrm{T} \text { )otal } \\
\text { Quantity }\end{array}$ & Unit & $\begin{array}{l}\text { Begin } \\
\text { Year }\end{array}$ & $\begin{array}{l}\text { End } \\
\text { Year }\end{array}$ & Fore & $\begin{array}{l}\text { Samp } \\
\text { les? }\end{array}$ & $\begin{array}{c}\text { Minimum } \\
\text { Value/\#Samp }\end{array}$ & $\begin{array}{r}\text { Maximum } \\
\text { Value/STD }\end{array}$ & $\begin{array}{l}\text { Basis for } \\
\text { Uncertainty }\end{array}$ \\
\hline$J-238$ & Sorbed on waste solids. & Probably oxides. & T. .01800000000000 & CI & 1994 & 2003 & $Y$ & N & $-50 \%$ & $+50 \%$ & Professional judgment. \\
\hline $4 n-54$ & Sorbed on waste solids. & Probably oxides. & T. .17000000000000 & CI & 1994 & 2003 & $Y$ & N & & & \\
\hline & & & & & & & & & & & \\
\hline & & & & & & & & & & & \\
\hline & & & & & & & & & & & \\
\hline & & & & & & & & & & & \\
\hline & & & & & & & & & & & \\
\hline & & & & & & & & & & & \\
\hline & & & & & & & & & & & \\
\hline & & & & & & & & & & & \\
\hline & & & & & & & & & & & \\
\hline & & & & & & & & & & & \\
\hline & & & & & & & & & & & \\
\hline & & & & & & & & & & & \\
\hline & & & & & & & & & & & \\
\hline
\end{tabular}

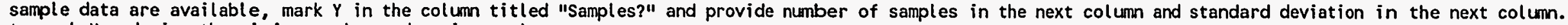
ot, mark $N$ and give the minimum value and maximum value.

tional information or explanations (indicate pertinent contaminant)

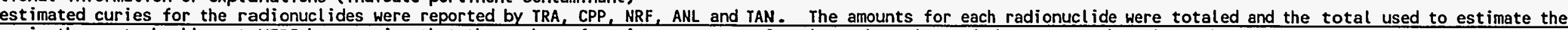

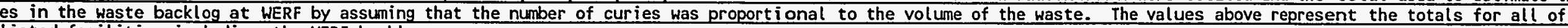
listed facilities including the WERF backlog. 
- Type of source of information: check box)

] RWMIS [ ] other database

] sample analysis data

operating records $[\mathrm{X}]$ interview

] expert judgment $[\mathrm{X}]$ reports

$x]$ generator forecasts

] other

- Do the estimates of contaminant uantities in Part $C$ and $D$ represent:

$\mathrm{X}$ ] best estimate

] worst case

] other

i. Do the data conflict with RWMIS? (Historical or Present Data only) $x]$ no

] yes

'. Major unknowns in inventories of :ontaminants:

Incertainty in future programs that will roduce the waste. Uncertainty relative to startup of WERF.
2. Details concerning source (names, report no., dates, etc.) Report, WERF SAR, Vol.1, WM-F1-81-026, Rev. 9, June 1993 Solid Radioactive Waste Forecast (RS1-018-93) prepared by J.R. Hitz and approved by R.L. Skinner, 11/15/93. Conversation with J.R. Hitz, a WINCO employee. See other sources on continuation page.

4. If other than best estimate, explain why:
8. Key assumptions used to deal with the unknowns: Assumed that the anticipated programs that will produce the waste are valid. Assumed that WERF or the other facility would begin incineration of this waste the first part of 1995. Disposal is assumed to begin in 1995. 
est Reactor Area (TRA) Radioactive Waste Forecasts, SJK-005-93, Nov. 17, 1993. Solid Radioactive aste Forecast, KAK-16-93, Nov。 15, 1993. NRF Forecasts for Waste sent to WERF, 995-2003-PRL-15-94, Aug. 9, 1994. Solid Radioactive Waste Forecast for TAN prepared by Mat anister, Nov. 18, 1993. Solid Radioactive Waste Forecast for TAN prepared by David Gibbon, Nov. 6, 1993. Data Input for RPDT for RWMC SDA, R.P. Grant, Sept. 30, 1994. 
DATA INPUT FOR HISTORICAL DATA TASK FOR RWMC SUBSURFACE DISPOSAL AREA

\begin{abstract}
RT A - GENERAL INFORMATION PDT - 308
\end{abstract}
Preparer: Rhodes, Donald W.

Generator: WER

rea or contractor - use code from attached list)

Number of waste stream from this facility: 1P

Type of radioactive waste (check box):

] TRU or suspect TRU

:] LLW

] non-radioactive

Actual years disposed of at SDA: :arting year 1994 Ending year 2003
2. Date prepared: $08 / 22 / 94$

4. Particular facility: MET

(building number - use code from attached list)

6. Waste stream:

This waste stream is metal in various forms that is sent to WERF for size reduction by cutting to reduce the space it requires for burial in the RWMC.

9. Waste stream volume:

Amount $\quad 2522.0000$ Units Cubic meters.

check box: [ ] annual or [X] total over all years

Check box: $[\mathrm{X}]$ container volume or [] waste volume

). Comments (specify number of pertinent question): 9. This is the volume after size reduction. Prior to size reduction the volume was estimated to be $12637 \mathrm{M} 3$. Size reduction is assumed to produce a 5:1 reduction in volume. The facilities that produced the waste are CPP, TRA, NRF, TAN, ANL and a backlog of waste currently stored at WERF. 
General physical form (see attached list) 2. Details on physical form(particularly confinement related) her scrap metals. pipe, structural metals, metal tanks, and machinery.

] other (specify)

Chemical form:

ainless steel, carbon steel, and uminum.

Waste container type (see attached list) oden box.

Comments (specify number of pertinent question):
4. Inner packaging: [ ] plastic bag [ ] plastic liner [ ] metal liner [X] none [ ] other (specify)

6. Other characteristics of interest: 
$r$ each contaminant, complete at least one line on the following table. If any entries for that contaminant vary by year, fill out additional lines as needed

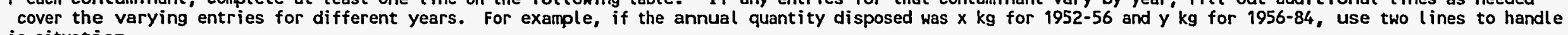
is situation.

\begin{tabular}{|c|c|c|c|c|c|c|c|c|c|c|c|}
\hline Radionucl ide & Physical Form & Chemical form & $\begin{array}{l}\text { (A)nnual } /(T) \text { otal } \\
\text { Quantity }\end{array}$ & Unit & $\begin{array}{l}\text { Begin } \\
\text { Year }\end{array}$ & $\begin{array}{l}\text { End } \\
\text { Year }\end{array}$ & $\begin{array}{l}\text { Fore } \\
\text { cast }\end{array}$ & $\begin{array}{l}\text { Samp } \\
\text { les? }\end{array}$ & $\begin{array}{c}\text { Minimum } \\
\text { Value/\#Samp }\end{array}$ & $\begin{array}{r}\text { Maximum } \\
\text { Value/STD }\end{array}$ & $\begin{array}{l}\text { Basis for } \\
\text { Uncertainty }\end{array}$ \\
\hline Ru-106 & Sorbed on waste solids. & Probably oxides. & T. .26000000000000 & CI & 1994 & 2003 & $\mathbf{Y}$ & N & & & \\
\hline Rh-106 & Sorbed on waste solids. & Probably oxides. & $T .26000000000000$ & CI & 1994 & 2003 & $Y$ & $N$ & & & \\
\hline Eu-152 & Sorbed on waste solids. & Probably oxides. & $T .05000000000000$ & $\mathrm{Cl}$ & 1994 & 2003 & $Y$ & $\mathbf{N}$ & & & \\
\hline Cs -134 & Sorbed on waste solids. & Probably oxides. & $T .75000000000000$ & $\mathrm{Cl}$ & 1994 & 2003 & $y$ & $N$ & & & \\
\hline Cs -137 & Sorbed on waste solids. & Probably oxides. & T 18.130000000000 & $\mathrm{CI}$ & 1994 & 2003 & $Y$ & N & & & \\
\hline Ce- 144 & Sorbed on waste solids. & Probably oxides. & T. .70000000000000 & $\mathrm{Cl}$ & 1994 & 2003 & $Y$ & $N$ & & & \\
\hline $\operatorname{Pr}-144$ & Sorbed on waste solids. & Probably oxides. & $T .70000000000000$ & CI & 1994 & 2003 & $\gamma$ & N & & & \\
\hline Eu-155 & Sorbed on waste solids. & Probably oxides. & T.17000000000000 & CI & 1994 & 2003 & $Y$ & N & & & \\
\hline $1-129$ & Sorbed on waste solids. & Probably oxides. & $T .00700000000000$ & CI & 1994 & 2003 & $Y$ & N & $-50 \%$ & $+50 \%$ & Professional judgment. \\
\hline
\end{tabular}

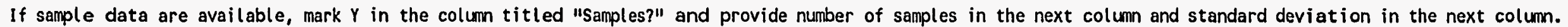
f not, mark $N$ and give the minimum value and maximum value,

dditional information or explanations (indicate pertinent contaminant)

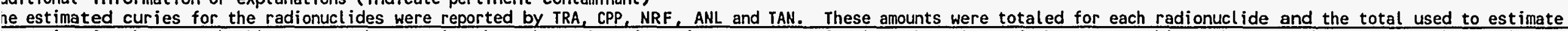

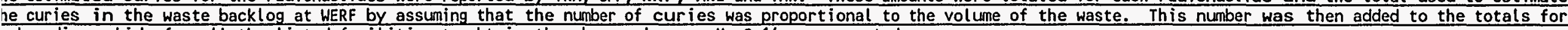
ach radionuclide for all the listed facilities to obtain the above values. No C-14 was reported. 
or each contaminant, complete at least one line on the following table. If any entries for that contaminant vary by year, fill out additional lines as needed

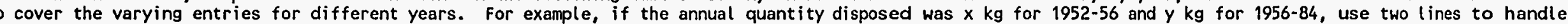
is situation.

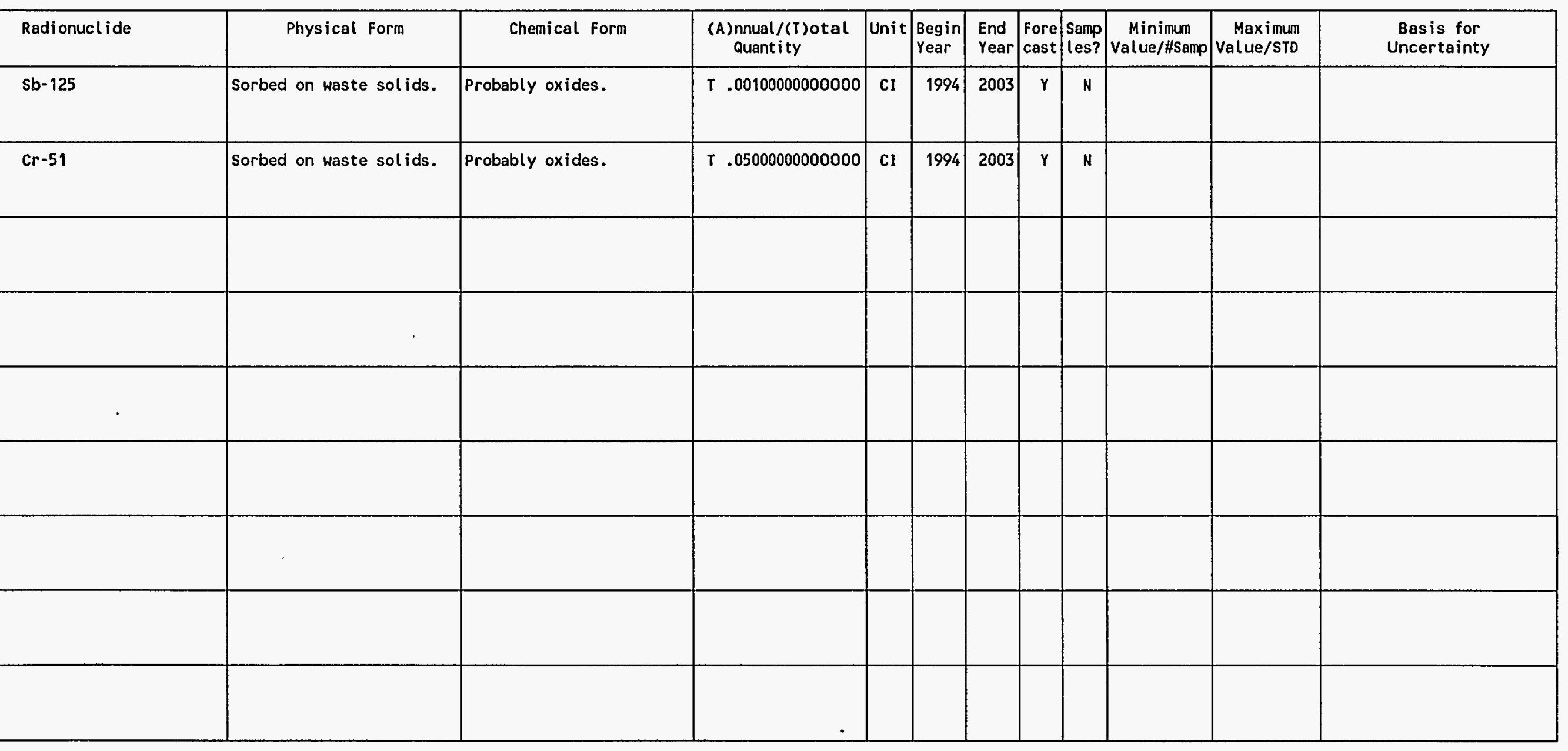

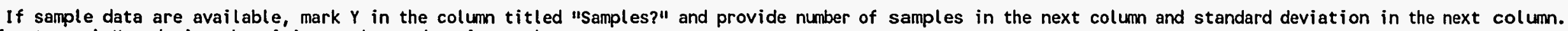
not, mark $N$ and give the minimum value and maximum value.

ditional information or explanations (indicate pertinent contaminant)

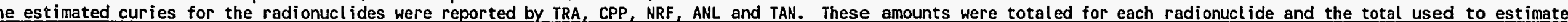

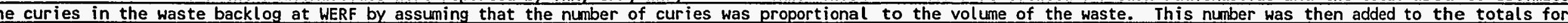
ach radionuclide for all the listed facilities to obtain the above values. No C-14 was reported. 
Type of source of information: heck box)

] RWMIS [ ] other database

] sample analysis data

operating records [ ] interview

] expert judgment $[X]$ reports

generator forecasts

] other

Do the estimates of contaminant lantities in Part $C$ and $D$ represent:

] best estimate

worst case

] other

Do the data conflict with RWMIs? (Historical or Present Data only)

] no

] yes

Major unknowns in inventories of ontaminants:

certain about future programs that will coduce the waste. Uncertain about startup te for WERF.
2. Details concerning source (names, report no., dates, etc.) Report WERF SAR, Vol.1, WM-F1-81-026, Rev.9, June 1993 Solid Radioactive Waste Forecast (RST-018-93) prepared by J.R. Hitz and approved by R.L. Skinner, 11/15/93. Conversation with J.L. Butler. See other sources on continuation page.

4. If other than best estimate, explain why:

\section{If yes, explain why:}

8. Key assumptions used to deal with the unknowns: Assumed that the anticipated programs that will produce the waste are valid. Assumed that WERF size reduction will begin operating the early part of 1995. Disposal assumed to begin in 1995. 
st Reactor Area (TRA) Radioactive Waste Forecasts - SJK-005-93, Nov. 17, 1993. Solid Radioactive te Forecasts KAK-16-93, Nov. 15, 1993. NRF Forecasts for Waste sent to WERF, 1995-2003-PRL-15-94, g. 9, 1994. Solid Radioactive Waste Forecast for TAN, prepared by Mat Banister, Nov. 18, 1993. id Radioactive Waste Forecast for TAN prepared by David Gibbon, Nov. 16, 1993 . Data Input for DT for RWMC SDA, R.P. Grant, Sept. 30, 1994. 
.. Preparer: Rhodes, Donald W.

1. Generator: WMC

'area or contractor - use code from attached list)

;. Number of waste stream from this facility: 2R

7. Type of radioactive waste (check box): ] T TRU or suspect TRU

: X] LLW

] non-radioactive

3. Actual years disposed of at SDA: starting year 1984 Ending year 1990
2. Date prepared: $07 / 05 / 94$

4. Particular facility: WMC

(building number - use code from attached list)

6. Waste stream:

7. The radionuclides are transuranic elements, normally in concentrations $<10 \mathrm{nci} / \mathrm{g}$. A few shipments (approximately 14) had concentrations between 10 and $100 \mathrm{nCi} / \mathrm{g}$, and one shipment slightly exceeded $100 \mathrm{nCi} / \mathrm{g}$. All of this waste was produced at the Rocky Flats operation and shipped to the INEL for disposal.

9. Waste stream volume:

Amount

246.5000 Units Cubic meters.

Check box: [] annual or [X] total over all years

Check box: $[\mathrm{X}]$ container volume or [ ] waste volume

10. Comments (specify number of pertinent question): 
General physical form (see attached 1ist) 2. Details on physical form(particularly confinement related) her scrap metals.

\section{] other (specify)}

\section{Chemical form:}

Waste container type (see attached list) tal barrel.

Comments (specify number of pertinent question):
4. Inner packaging: [ ] plastic bag [ ] plastic liner

[ ] metal liner [X] none [ ] other (specify)

6. Other characteristics of interest: 
- each contaminant, complete at least one line on the following table. If any entries for that contaminant vary by year, fill out additional lines as needed

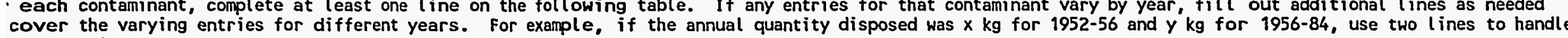
s situation.

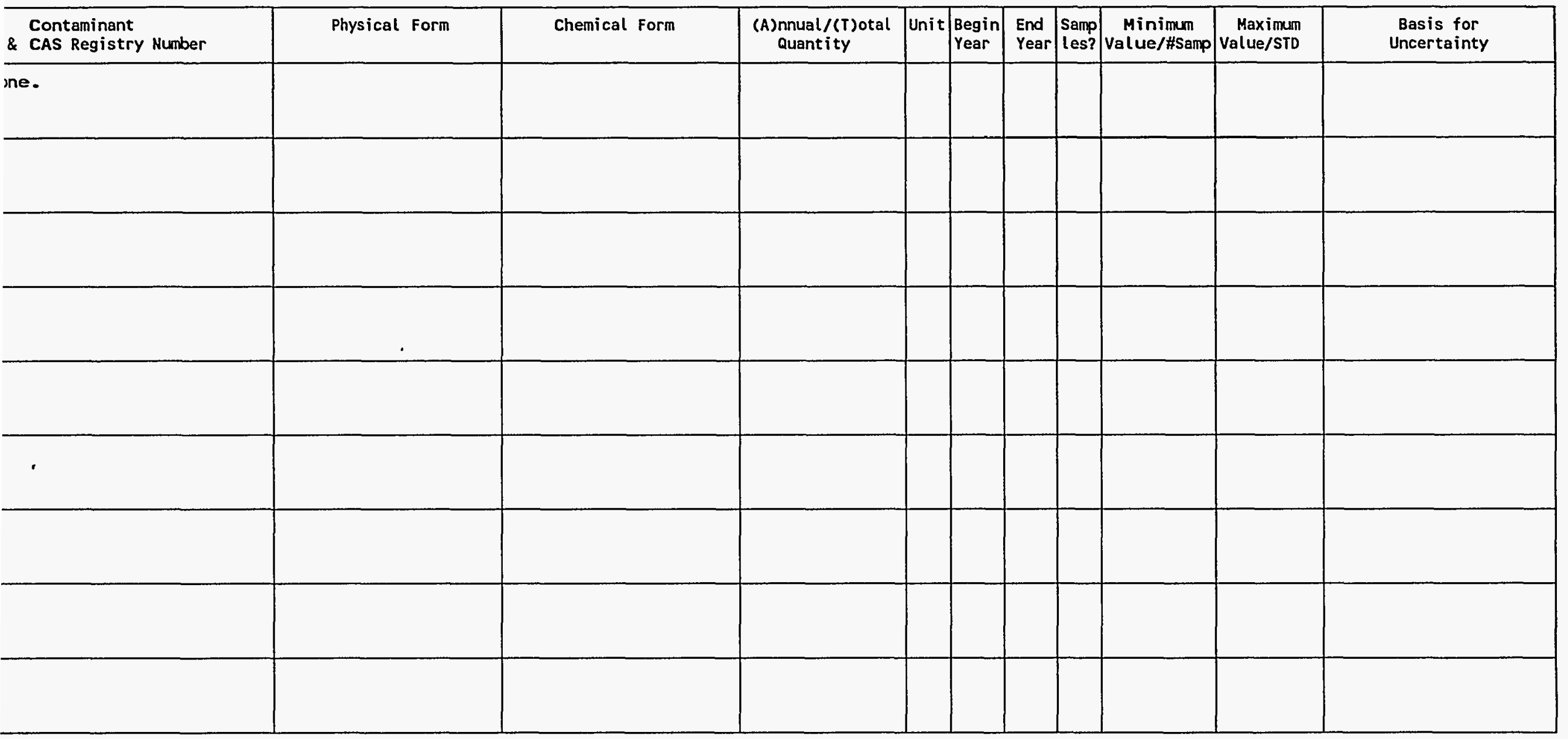

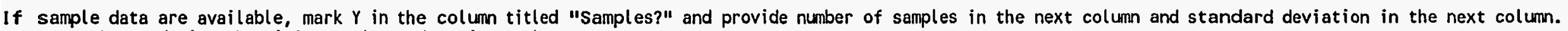
not, mark $N$ and give the minimum value and maximum value.

ditional information or explanations (indicate pertinent contaminant) 
- Type of source of information: check box)

X] RWMIS $[\mathrm{X}]$ other database

] sample analysis data

] operating records [ ] interview

] expert judgment [ ] reports

] other

- Do the estimates of contaminant uantities in Part $C$ and $D$ represent:

$\mathrm{X}$ ] best estimate

] worst case

] other

- Do the data conflict with RWMIS? (Historical or Present Data only)

$\mathrm{X}$ ] no

] yes

- Major unknowns in inventories of ontaminants:

11 waste is in solid form.
2. Details concerning source (names, report no., dates, etc.) Curie content is determined from isotopic analysis of smear samples. The data is used to develop an $\mathrm{mR} / \mathrm{hr}$ to curie content conversion factor. The $+/-25 \%$ uncertainty is due to measurements with G-M instruments. * These totals include 7 backlog cargo container waste only.

4. If other than best estimate, explain why:

6. If yes, explain why:

8. Key assumptions used to deal with the unknowns: 
DATA INPUT FOR HISTORICAL DATA TASK FOR RWMC SUBSURFACE DISPOSAL AREA

ART A - GENERAL INFORMATION PDT - 292

Preparer: Rhodes, Donald W.

Generator: WMC

area or contractor - use code from attached list)

Number of waste stream from this facility: IP

Type of radioactive waste (check box):

] TRU or suspect TRU

LLW

] non-radioactive

Actual years disposed of at SDA: tarting year 1994 Ending year 2003
2. Date prepared: 08/02/94

4. Particular facility: ALL

(building number - use code from attached list)

6. Waste stream:

Miscellaneous waste, including cleanup materials, resulting from operations conducted at the Radioactive Waste Management Complex.

9. Waste stream volume:

Amount 161.1000 Units Cubic meters.

Check box: [ ] annual or $[\mathrm{X}]$ total over all years Check box: [X] container volume or [ ] waste volume

Comments (specify number of pertinent question):

4. This stream includes waste from all of the buildings and other structures at the RWMC. 
General physical form (see attached list) 2. Details on physical form(particularly confinement related) her scrap metals. Waste will be placed in containers for disposal without any special treatment to inhibit leachability, because the radionuclide content is expected to be very low.

4. Inner packaging: [ ] plastic bag [X] plastic liner

[ ] metal liner [ ] none [ ] other (specify)

Chemical form:

tal (carbon and stainless steels,)

llulose (wood and paper), plastic, uminosilicates (soil), and silica lass).

Waste container type (see attached list) oden box.

Comments (specify number of pertinent question):

1. Also $21,41,42,43$, and 44 .

5. Also BXC and DMP. 


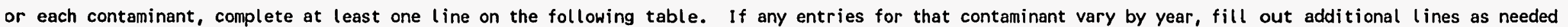

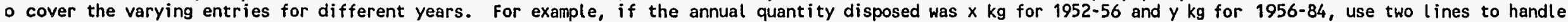
his situation.

\begin{tabular}{|c|c|c|c|c|c|c|c|c|c|c|c|}
\hline Radionuclide & Physical Form & Chemical form & $\begin{array}{l}\text { (A)nnual/(T)otal } \\
\text { Quantity }\end{array}$ & Unit & $\begin{array}{l}\text { Begin } \\
\text { Year }\end{array}$ & $\begin{array}{l}\text { End } \\
\text { Year }\end{array}$ & $\begin{array}{l}\text { Fore } \\
\text { cast }\end{array}$ & $\begin{array}{l}\text { Samp } \\
\text { les? }\end{array}$ & $\begin{array}{c}\text { Minimum } \\
\text { Value/\#Samp }\end{array}$ & $\begin{array}{r}\text { Maximum } \\
\text { Value/STD }\end{array}$ & $\begin{array}{l}\text { Basis for } \\
\text { Uncertainty }\end{array}$ \\
\hline Co-60 & Sorbed on waste solids. & Probably oxides. & $T .26000000000000$ & CI & 1994 & 2003 & $\mathbf{Y}$ & N & & & \\
\hline Fe-55 & Sorbed on waste solids. & Probably oxides. & T. .07000000000000 & CI & 1994 & 2003 & $Y$ & N & & & \\
\hline $\mathrm{Ni}-63$ & Sorbed on waste solids. & Probably oxides. & T. .02000000000000 & CI & 1994 & 2003 & Y & N & & & \\
\hline Ru-106 & Sorbed on waste sol ids. & Probably oxides. & T. .00100000000000 & CI & 1994 & 2003 & $Y$ & N & & & \\
\hline Pu-239 & Sorbed on waste solids. & Probably oxides. & T. .00080000000000 & CI & 1994 & 2003 & $Y$ & N & $-50 \%$ & $+50 \%$ & Professional judgment. \\
\hline$A m-241$ & Sorbed on waste solids. & Probably oxides. & T. .00030000000000 & CI & 1994 & 2003 & $\mathbf{Y}$ & $\mathrm{N}$ & $-50 \%$ & $+50 \%$ & Professional judgment. \\
\hline & & & & & & & & & & & \\
\hline & & & & & & & & & & & \\
\hline & & & & & & & & & & & \\
\hline & & & & & & & & & & & \\
\hline & & & & & & & & & & & \\
\hline & & & & & & & & & & & \\
\hline
\end{tabular}

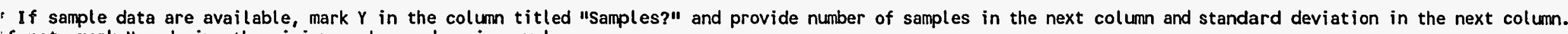
f not, mark $N$ and give the minimum value and maximum value.

idditional information or explanations (indicate pertinent contaminant) 
L. Type of source of information: (check box)

\section{[ ] RWMIS [ ] other database \\ ] sample analysis data \\ ] operating records [ ] interview \\ ] expert judgment [ ] reports \\ [X] generator forecasts \\ [ ] other}

3. Do the estimates of contaminant

quantities in Part $C$ and $D$ represent:

$[\mathrm{X}]$ best estimate

[ ] worst case

] other

5. Do the data conflict with RWMIS? (Historical or Present Data only) [X] no

[ ] yes

7. Major unknowns in inventories of contaminants:

Chemical and physical forms not identified.
2. Details concerning source (names, report no., dates, etc.) Solid Radioactive Waste Forecast prepared by D.M. Miley, oct. 18,1993 and approved by J.R. Bishoff.

4. If other than best estimate, explain why:

6. If yes, explain why:

8. Key assumptions used to deal with the unknowns:

Assumed that chemical and physical forms would be similar to waste produced in the past. 